\title{
Energy-Efficient Mortgages and Home Energy Rating Systems: A Report on the Nation's Progress
}

Barbara C. Farhar and Jan Eckert

\section{NRE}

National Renewable Energy Laboratory

(formerly the Solar Energy Research Institute)

1617 Cole Boulevard

Golden, Colorado 80401-3393

Operated for the

U.S. Department of Energy

by the Midwest Research Institute

under Contract No. DE-AC02-83CH10093

Prepared under Task No. AS135440

September 1993 


\section{NOTICE}

NOTICE: This report was prepared as an account of work sponsored by an agency of the United States government. Neither the United States govemment nor any agency thereof, nor any of their employees, makes any warranty, express or implied, or assumes any legal liability or responsibility for the accuracy, completeness, or usefulness of any information, apparatus, product. or process disclosed, or represents that its use would not infringe privately owned rights. Reference herein to any specific commercial product, process, or service by trade name, trademark, manufacturer, or otherwise does not necessarily constitute or imply its endorsement, recommendation, or favoring by the United States government or any agency thereof. The views and opinions of authors expressed herein do not necessarily state or reflect those of the United States government or any agency thereof.

$$
\begin{aligned}
& \text { Printed in the United States of America } \\
& \text { Available from: }
\end{aligned}
$$

National Technical Information Service

U.S. Department of Commerco 5285 Port Royal Road Springfield, VA 22161

Price: Microfiche AOI

$$
\text { Printed Copy } A 13
$$

Codes are used for pricing all publications. The code is determined by the number of pages in the publication. Information pertaining to the pricing codes can be found in the current issue of the following publications which are generally available in most libraries: Energy Research Abstracts (ERA): Government Reports Announcements and Index (GRA and I); Scientific and Technical Abstract Reports (STAR); and publication NTIS-PR-360 available from NTIS at the above address. 


\section{DISCLAIMER}

Portions of this document may be illegible in electronic image products. Images are produced from the best available original document. 


\section{Preface}

The work reported here was supported by the Office of Building Technologies, Office of Energy Efficiency and Renewable Energy. This report covers the work of the National Collaborative itself and assesses progress since the Collaborative reached its consensus agreements on widespread implementation of EEMs linked with HERS to finance residential energy improvements in the United States. The Collaborative recommended that this report on progress should be prepared a year after $A$ Blueprint for Action's release.

This project could not have occurred without the active support and involvement of Mary-Margaret Jenior, Program Manager and John P. Millhone, Deputy Assistant Secretary, U.S. Department of Energy, and the active cooperation of the U.S. Department of Housing and Urban Development. The members of the National Collaborative on Home Energy Rating Systems (HERS) and Energy-Efficient Mortgages (EEMs), and its technical advisory committees, contributed significantly to its completion. Without their dedication and commitment to a daunting task, progress would have been slowed. The active efforts of many persons in states across the country are also acknowledged. These individuals and their organizations have nurtured the idea of using mortgages to finance home energy improvements in their states and localities.

The National Collaborative wrote A Blueprint for Action (NREL/TP-261-4677, Review Draft, March 1992a). A companion volume, Going National with HERS and EEMs: Issues and Impacts (NREL/TP-261-4706, March 1992b), provides technical documentation to the Collaborative's efforts. It contains a collection of 55 technical issue papers and 13 special papers prepared by the members of the Collaborative and its technical advisory committees. In addition, Going National contains an extensive bibliography and a glossary, which were prepared by Collaborative members and staff.

This progress report was researched and prepared by the Analytic Studies Division of the National Renewable Energy Laboratory (NREL). Several staff at NREL have supported the efforts of the National Collaborative. These include Becky Baldwin, Ann Brennan, Vickie Corcoleotes, Mary Anne Dunlap, Barbara Glenn, Ron Judkoff, Mara Kalish, Irene Medina, Dorian McDuffie, Page Parker, Paula Pitchford, Lauren Raphael, Kim Rattley, Alyssa Tonelli, Joe Woodburn, and Kay Vernon. Members of stakeholder groups and state officials contributed significantly to the progress report by providing updates on relevant events within their purviews.

This progress report is one step in the ongoing process of increasing the use of energy-efficiency and renewable energy technologies in the nation's residential buildings.

Approved for the

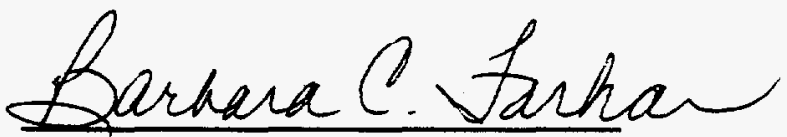

Barbara C. Farhar,

Senior Social Scientist

NATIONAL RENEWABLE ENERGY LABORATORY

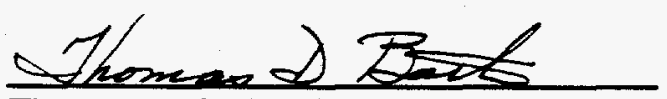

Thomas D. Bath, Director

Analytic Studies Division

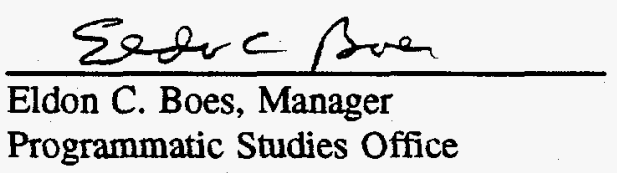




\section{Executive Summary}

This report summarizes progress throughout the nation in establishing voluntary programs linking home energy rating systems (HERS) and energy-efficient mortgages (EEMs). These programs utilize methods for rating the energy efficiency of new and existing homes and predicting energy cost savings so lenders can factor in energy cost savings when underwriting mortgages. The programs also encourage lenders to finance cost-effective energy-efficiency improvements to existing homes with low-interest mortgages or other instruments. The money saved on utility bills over the long term can more than offset the cost of such energy-efficiency improvements.

An effective program for financing energy improvements in housing is important if we are to achieve national energy-efficiency goals, strengthen our economy, and provide better housing for people who might not otherwise be able to afford it. The savings on monthly utility bills afforded by energy efficiency in housing could qualify as many as 250,000 new homebuyers per year for mortgages, according to a 1986 study by the Joint Center for Housing Studies.

\section{The National Collaborative on HERS and EEMs}

Early in 1991, the Department of Energy (DOE) and Housing and Urban Development (HUD) responded to the presidential mandate outlined in the National Energy Strategy of 1991 to "develop and encourage the voluntary acceptance of efficiency ratings and their use in home financing." The agencies also were charged with responsibility under federal legislation calling for a uniform plan to make housing more affordable through mortgage financing incentives for energy efficiency (the National Affordable Housing Act of 1990). DOE's Office of Building Technologies, in cooperation with HUD, established the National Collaborative on HERS and EEMs, composed of members from 25 different stakeholder organizations. Following 10 meetings in 1991 and 1992, the Collaborative released A Blueprint for Action, which contained its findings and recommendations for a voluntary national HERS/EEMs program. The Collaborative recommended that a report on progress toward a national program be prepared.

In A Blueprint for Action, the Collaborative recommended that participation in HERS/EEMs programs should remain voluntary and that all stakeholders (lenders, the government, utilities, energy organizations, real estate professionals, and others) should be involved in formulating linkages between HERS and uniform EEMs processes.

The Collaborative's efforts, and the network formed by participation in it, have served as a springboard for activities by HUD, DOE, the Congress, the states, and some 25 stakeholder organizations to advance the use of HERS and EEMs. For example, the National Energy Policy Act of 1992 (EPAct) requires DOE to promulgate voluntary guidelines that encourage uniformity in HERS in consultation with other agencies, HERS providers, builders, utilities, and others. EPAct requirements for HERS are similar to the recommendations of the National Collaborative.

The Collaborative reached a consensus on many issues identified as important in developing and promoting uniform HERS/EEMs programs. Issues relating to EEMs included establishing qualifications for borrowers, home improvement loans, gathering data on default rates, and establishing suitable means for lender indemnification and property valuation. HERS issues included determining the accuracy and credibility of ratings, systems, and calculation or prediction tools used in the systems; maintaining fuel neutrality; treating occupant behavior; differentiating new and existing housing; accounting for renewables and technological advancements; and establishing quality control procedures and uniform rating scales.

Program implementation issues involved the need for a national data base, third-party financing, clarification of the roles to be played by various stakeholder groups, pilot tests, and determining the best 
mechanisms for advancing HERS/EEMs programs. Awareness issues included the need for public information programs as well as adequate training of builders, lenders, real estate professionals, utility personnel, and consumers in the HERS/EEMs process.

\section{Legislation}

The acceleration and linkage of the HERS and EEMs programs, which have existed in various states and localities and at the national level for more than a decade, were supported by three bills signed in 1992: the National Energy Policy Act (EPAct), the Housing and Community Development Act, and the Veterans Home Loan Program Amendments. EPAct directs DOE to develop voluntary guidelines to encourage uniformity in HERS. EPAct also stipulates that federally assisted new housing must comply with energyefficient building standards to receive mortgages insured by HUD/FHA under the National Housing Act of 1949. New housing subject to mortgages made by the FmHA (Farmers Home Administration) also must meet or exceed energy-efficient building standards. By October 1993, HUD/FHA and FmHA must establish these standards, which must meet or exceed the requirements of the Council of American Building Officials Model Energy Code, 1992 (CABO-MEC 92). The Housing and Community Development Act of 1992 calls for a five-state EEMs pilot program for existing housing that will be implemented by HUD/FHA. The states selected were Alaska, Arkansas, California, Vermont, and Virginia. The Veterans Home Loan Program Amendments of 1992 include provisions for a 50-state EEMs demonstration program to run through 1995.

\section{Energy-Efficient Mortgages}

Energy-efficient mortgages have been available for more than a decade but were actively marketed in only a few locales. EEMs programs allow homeowners to achieve utility-bill savings with modest increases in mortgage payments to cover the cost of adding energy-efficiency features to new and existing housing. Organizations currently offering, guaranteeing, purchasing, or insuring mortgages that recognize energy efficiency include the FHA, the Department of Veterans Affairs (DVA), the Farmers Home Administration (FmHA), the Federal National Mortgage Association (FNMA), and the Federal Home Loan Mortgage Corporation (FHLMC). At least 18 states have programs facilitating access to EEMs, and many more states are linking EEMs programs with various housing programs.

In addition to these efforts, a HUD Task Force assembled in June 1992 is developing a plan for achieving national uniformity for EEMs. The Task Force consists of representatives from HUD, DOE, FmHA, FNMA, and FHLMC, and consults with a number of other organizations, such as the National Association of Home Builders and the Mortgage Bankers Association. The EEM concepts that the HUD Task Force recommends could be tested in pilot programs to better understand and promote wider use of these mortgages.

EEMs are currently known to be offered at one or more lending institutions in at least 23 states. The HUD/FHA EEMs Pilot Program has already trained lenders in Alaska, Arkansas, California, Vermont, and Virginia.

\section{HERS Programs}

Performance-based and certification HERS programs are at least a decade old. They include the activities of Energy Rated Homes of America (ERHA), which has programs in nine states and several localities, and Good Cents and Super Good Cents Programs, which are used by 391 utilities in 32 states. Performance systems and certification programs are both used to assess the energy performance of a house. Performance systems, usually operated by state energy offices and nonprofit groups, estimate the relative energy efficiency of a house based on the thermal performance of the building envelope and the operation 
of heating, ventilating, air-conditioning, and water heating equipment. Certification programs are usually operated by utility companies, builders, and nonprofit groups. These programs perform inspections of energy efficiency that tend to focus on new electrically heated housing.

HERS programs exist in some form at the local or state level in 39 states. Programs are being developed or considered in other states, and 32 states are working actively to link HERS and EEMs. After the National Collaborative released A Blueprint for Action in 1992, a group representing mortgage finance, energy, builder, and consumer organizations and HERS providers formed the Home Energy Rating Systems (HERS) Council, which was incorporated in February 1993. The Council now contains more than 125 members from the utility industry, government, environmental and consumer groups, as well as members of the real estate, finance, and building industries. The Council's mission is to increase energy efficiency in housing by serving as an education and research resource and by establishing a nationwide voluntary program for accrediting systems and certifying tools that assign home energy-efficiency ratings, consistent with uniform guidelines, to access energy-efficient mortgages and other programs.

\section{Other Actions in Support of HERS and EEMs}

Besides federal and state legislation, some of the most significant activities initiated to date, and documented in the report, are

- Establishment of the HERS Council

- EEMs pilot programs by HUD/FHA, FNMA, DVA, and the Environmental Protection Agency (EPA)

- Edison Electric Institute's Three-E Program

- Analyses of HERS and EEMs data by ERHA, FNMA, and FHLMC

- Training initiatives for lenders (FHA/HUD) and raters (ERHA)

- Regional Good Cents Roundtables

- Contributions by agencies and stakeholders to EPAct and to national policy on HERS and EEMs.

The number of states now researching or actively planning HERS programs is rapidly increasing. This suggests that one of the barriers to broad implementation, lack of knowledge, is being overcome. Other barriers still remain, however, such as the lack of trained raters, lenders, appraisers, and real estate professionals.

In addition to voluntary HERS and EEM programs and linkages, many states are engaged in other activities that support the programs and promote energy efficiency. For example, at least 19 states have statewide building codes that meet or exceed standards established by the Council of American Building Officials (CABO) in 1989 for energy efficiency. And all 50 states have at least one utility residential demand-side management (DSM) program. These DSM programs, which encourage the efficient use of energy and the use of alternative energy sources to reduce the demand on conventional power plants, could become a critical institutional link between local and regional HERS and EEMs programs.

\section{Conclusions}

HERS and EEMs programs are interdependent in many ways. They need to be developed simultaneously if the country's goals for energy efficiency and greater diversity among energy resources are to be reached. It is noteworthy that so many of the recommendations of the National Collaborative on HERS and EEMs have already been implemented in legislation and other actions throughout the country. For example:

- EPAct and other relevant national and state legislation have relied on the ideas and the language of the National Collaborative's $A$ Blueprint for Action to set forth the requirements for energy efficiency ratings and EEMs programs 
- The HUD Task Force used the recommendations and technical papers of the National Collaborative as a starting point for its work

- The HERS Council is building on the Collaborative's work concerning technical issues, education and training needs, implementation, and HERS/EEMs linkage issues.

In addition, protocols for certifying HERS tools are being developed jointly for DOE by the National Renewable Energy Laboratory (NREL) and the HERS Council. HUD/FHA plans to continue an EEMs working group, after the HUD Task Force recommendations are made, to provide a venue for the federal entities that issue, guarantee, and insure mortgages and to monitor the results of EEMs pilot programs. HUD representatives have joined forces with their counterparts at DOE, NREL, and other organizations to form a HERS/EEMs management team that provides a forum for all relevant federal activities.

Many tasks and questions still remain. An analysis is needed of EEMs' impacts on utility costs, energy savings, environmental protection, housing affordability, and mortgage default rates. Consumers' preferences and behavior regarding energy investments and mortgage financing also should be researched. Important questions that must be answered include these: What EEMs incentives would capture the interest of real estate professionals, appraisers, home inspectors, and lenders? How will HERS programs be related to weatherization and other housing programs? Should utility DSM programs be developed in partnership with EEMs, and, if so, how? What action and information is needed to develop data for use in valuation procedures? How will the incumbents be recruited and trained for the new positions and specialties that HERS/EEMs programs will require (e.g., raters, lenders, real estate professionals, appraisers, and others)? How can consumers best be made aware of the potential benefits of EEMs/HERS and motivated to avail themselves of them in their own housing decisions?

The institutional mechanisms that can provide answers to such questions are now in place. And one advantage of the entire effort is that the organizations that have to make the process work are the ones designing it. Progress is advancing on many fronts, and this testifies to the widespread interest in the potential of HERS and EEMs to save energy, protect the environment, improve the quality and affordability of housing, and strengthen the nation's economy.

The next major national milestones in the path toward nationwide financing of residential energy efficiency improvements will be

- HUD's Report to Congress on the EEMs pilots by April 1995, and the expansion of FHA's EEMs program to include the entire nation, as well as new housing

- The release of the HUD Section 946 Task Force report

- DOE's Report to Congress by October 1995 on the residential energy-efficiency guidelines and technical assistance program, including a recommendation on the feasibility of requiring a minimum energy-efficiency rating as a prerequisite to receiving federally assisted, guaranteed, or insured mortgages.

Momentum for significant progress and resolution of issues has been established. Another assessment of national progress would probably be useful by the end of 1995 . 


\section{Contents}

Introduction and Background

Benefits of a National EEMs/HERS Program . . . . . . . . . . . . . . . 1

EEMs Programs Available . . . . . . . . . . . . . . . . . . . . . . 2

HERS Performance-based Systems and Certification Programs $\ldots \ldots \ldots \ldots \ldots \ldots 2$

Purpose and Organization of the Report $\ldots \ldots \ldots \ldots \ldots \ldots \ldots \ldots \ldots$

The National Collaborative and A Blueprint for Action $\ldots \ldots \ldots \ldots \ldots \ldots \ldots$

Dissemination and Distribution of the Collaborative's Reports $\ldots \ldots \ldots \ldots \ldots \ldots \ldots$

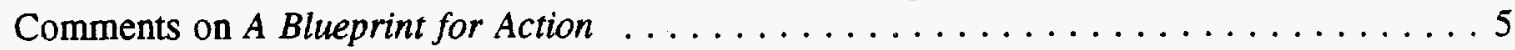

Future of Collaborative Efforts $\ldots \ldots \ldots \ldots \ldots \ldots \ldots \ldots \ldots \ldots \ldots$

The HUD Section 946 Task Force $\ldots \ldots \ldots \ldots \ldots \ldots \ldots \ldots \ldots \ldots \ldots$

The Home Energy Rating System Council $\ldots \ldots \ldots \ldots \ldots \ldots \ldots \ldots$

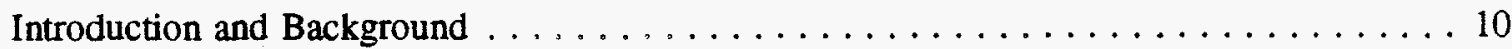

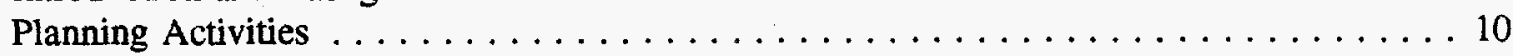

Membership . . . . . . . . . . . . . . . . . . . . . . . . . . 11

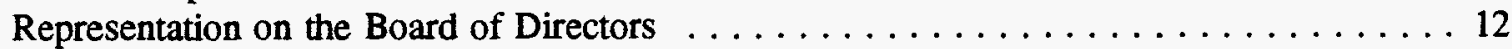

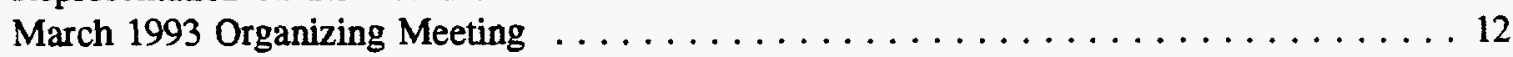

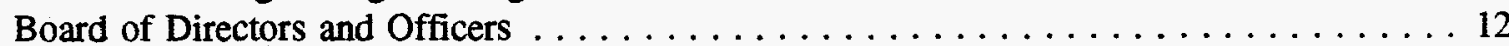

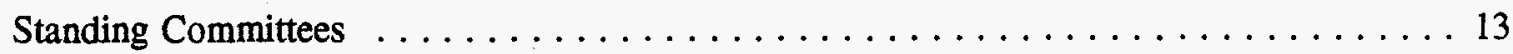

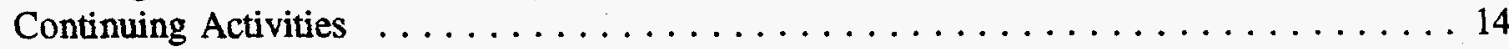

Legislation in Support of HERS and EEMs $\ldots \ldots \ldots \ldots \ldots \ldots \ldots \ldots \ldots \ldots$

Residential Energy-Efficiency Rating Guidelines $\ldots \ldots \ldots \ldots \ldots \ldots \ldots \ldots \ldots \ldots$

HUD/FHA EEMs Pilot Tests in Five States . . . . . . . . . . . . . . . . . 16

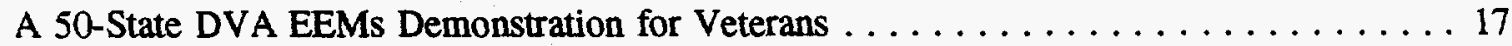

Expansion of HUD Section 946 Task Force Membership $\ldots \ldots \ldots \ldots \ldots \ldots \ldots \ldots$

Mandating HUD and FmHA Use of More Energy-Efficient

Building Standards for New Housing Mortgages . . . . . . . . . . . . . . . . 19

State Legislative Activity in Support of HERS and EEMs . . . . . . . . . . . . . . 19

Residential Energy Efficiency Rating Guidelines $\ldots \ldots \ldots \ldots \ldots \ldots \ldots \ldots \ldots \ldots \ldots$

Pilot Tests for Energy-Efficient Mortgages $\ldots \ldots \ldots \ldots \ldots \ldots \ldots \ldots \ldots \ldots \ldots$

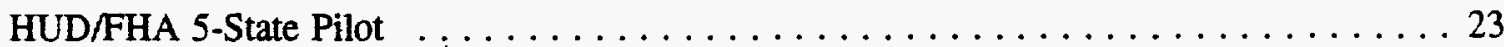

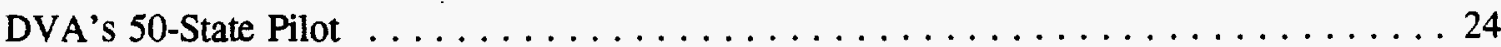

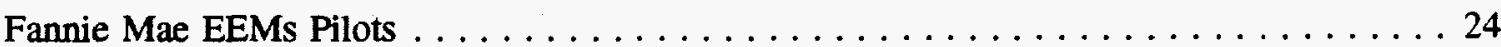

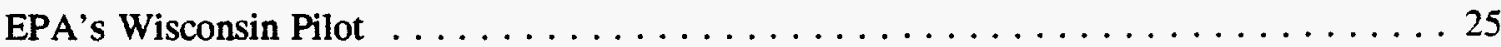

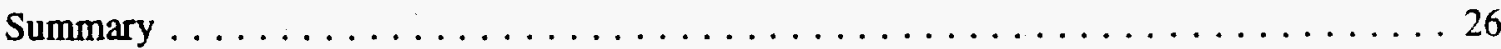


Contents (Continued)

\section{$\underline{\text { Page }}$}

Actions in Support of HERS and EEMs $\ldots \ldots \ldots \ldots \ldots \ldots \ldots \ldots \ldots \ldots$

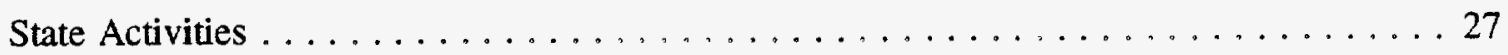

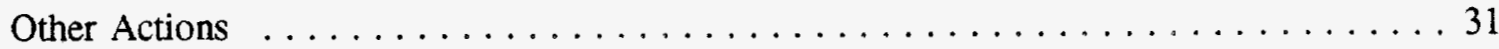

Evaluation of HERS and EEMs Activities $\ldots \ldots \ldots \ldots \ldots \ldots \ldots \ldots \ldots \ldots$

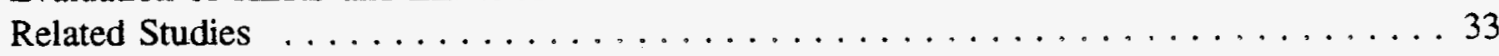

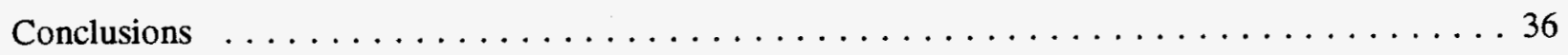

Bibliography $\ldots \ldots \ldots \ldots \ldots \ldots \ldots \ldots \ldots \ldots \ldots \ldots \ldots \ldots \ldots \ldots$

Appendixes

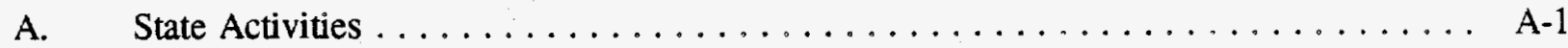

B. Actions by Stakeholder Organizations Represented in the National Collaborative . . . . . B B-1

C. Summary of Organizational Comments on A Blueprint for Action . . . . . . . . . C-1

D. Recent Articles on the National Collaborative, HERS, and EEMs (1991-1993) ...... D-1

E. Text of A Blueprint for Action $\ldots \ldots \ldots \ldots \ldots \ldots \ldots \ldots \ldots \ldots \ldots \ldots$ 


\section{List of Tables}

Page

1 Comparison of National Collaborative Recommendations and EPAct

Requirements on Residential Energy Efficiency Ratings

2 Evaluation Requirements - HUD/FHA EEMs Pilots

34

3 Evaluation Requirements - DOE Residential Energy Efficiency Ratings Voluntary

Guidelines

Summary of EEMs/HERS Activities by State

5 Actions by National-level HERS/EEMs Stakeholder Groups,

Spring 1992-Spring 1993

\section{List of Figures}

Page

1 States with HERS programs (either statewide, local-area, or both) (as of 09/93) . . . . 28

2 States with one or more lending institutions that facilitate access to EEMs (as of 09/93) . . 30 


\section{Introduction and Background}

Cost-effective energy-efficiency technologies ${ }^{1}$ exist today to reduce dramatically the $\$ 100$ billion spent in the United States each year on utility-supplied energy in housing. Use of these technologies has been slowed because home builders and buyers want to minimize the "up-front" cost of a residential property, even at the expense of future savings. Mortgage loan practices reinforced this tendency because they failed to consider the total cost of owning an energy-efficient home when energy expenses are added to mortgage, insurance, and tax payments.

Although energy-efficient mortgages (EEMs) have been in existence since 1980, no widely accepted mechanism to finance the cost of energy improvements existed. These programs have been underutilized. Few buyers and lenders are aware of the EEM option. For lenders, EEMs are often seen as representing additional paperwork. The existing programs lack uniformity in formats and procedures. The mortgage community is unsure of the reliability of the promised energy savings. The relationship between energy performance and housing values remains undocumented. And because homeowners are unaware of EEMs and do not inquire about them, this is perceived as a lack of market interest.

Similarly, home energy rating systems (HERS) and certification programs have been in existence for more than a decade. The Energy Rated Homes of America organization has statewide programs in nine states, and local-area programs in a few others. Several other states are beginning their own programs, as this report documents. The cities of Fort Collins, $\mathrm{CO}$ and Austin, TX have their own rating programs. The Good Cents and Super Good Cents certification programs are carried out in nearly 400 utilities nationwide, especially in the South and the Pacific Northwest. Nevertheless, these programs, while used, have not been adequately linked with EEMs to create a viable system.

\section{Benefits of a National EEMs/HERS Program}

More than 8.5 million loans worth $\$ 894$ billion were originated in the United States in 1992 (Mortgage Bankers Association of America 1993). Their average value was $\$ 105,962$. With EEMs actively used, perhaps some 250,000 families could qualify as first-time homeowners each year who would not otherwise have qualified (Joint Center for Housing Studies 1986). The increase in the pool of potential buyers would mean more homes could be built and purchased. At the same time, residential energy demand would be decreased and the increment in environmental pollution associated with the production, distribution, and consumption of that energy would be avoided. The number of jobs associated with energy rating, lending, real estate, inspection, manufacture, and installation of energy-improvement measures would probably increase. The intention of existing EEMs legislation would be more adequately met.

EEMs are intended to result in energy savings in residential buildings. Total U.S. residential energy consumption in 1993 was 16.9 quads (Battelle 1993). DOE has estimated that a $30 \%$ increase in the efficiency of all buildings by 2010 would have the following impacts:

- Save 10 quads of energy in the year 2010 (relative to a 1993 reference case)

- Reduce consumer energy expenditures by $\$ 75$ billion in 2010 (in 1990 dollars)

\footnotetext{
l"Energy efficiency," as used throughout this report, is intended to include both energy-efficiency measures (such as insulation and low-emissivity windows) and renewable energy technologies (such as passive solar design and solar domestic hot water systems), the use of which reduces a home's consumption of fuel oil and utility-supplied energy.
} 
- Free investment capital for other uses (80 1000-MW powerplants would not have to be built over the next 20 years)

- Reduce buildings-related emissions of $\mathrm{CO}_{2}$ by 120 million metric tons in 2010 .

However, the specific impacts of EEMs have not been thoroughly analyzed and are not yet well understood. Little is known about potential consumer acceptance and use of EEMs. More systematic analysis will provide the kinds of information needed to document advantages and to reduce barriers to the use of EEMs and HERS.

\section{EEMs Programs Available}

Existing EEMs programs increase the affordability of housing by enabling homeowners to achieve savings on their utility bills with only modest increases in mortgage payments. They allow borrowers to add the cost of cost-effective energy improvements to mortgages, thereby providing the longer term, lower interest rate, and tax benefit of mortgage financing. The increase in mortgage payments is typically more than offset by savings on energy costs.

The four existing EEMs programs are:

- The Federal Housing Administration (FHA) of the Department of Housing and Urban Development (HUD) insures home mortgages for qualifying home buyers. FHA/HUD allows an increase in the loan amount within certain limits and "stretches" the income qualifying ratios for mortgages on new homes meeting specific guidelines for energy efficiency.

- The Department of Veterans Affairs (DVA) guarantees mortgages for qualifying veterans with little or no down payment, with the maximum loan amount determined by the participating lender. DVA allows an increase in the mortgage amount for energy-related improvements, within certain limits and with appropriate documentation.

- The Farmers Home Administration (FmHA) requires conformance with its thermal performance construction standards for all its loans, which are provided directly to qualifying borrowers in rural areas under 10,000 population. FmHA will loan an additional amount for energy improvements that are economically justified.

- The Federal National Mortgage Association (FNMA or Fannie Mae) and the Federal Home Loan Mortgage Corporation (FHLMC or Freddie Mac) have developed and promulgated to lenders criteria for mortgages they are willing to buy. Lenders, in turn, can offer such loans to borrowers. Fannie Mae has offered a 2\% ratio stretch for new residential construction meeting voluntary consensus building standards, known as CABO-MEC (Council of American Building Officials Model Energy Code).

Existing EEM programs are diverse rather than uniform and easy to use. Although each of the programs makes EEMs available, each follows its own rules, uses its own forms, and has its own procedures.

\section{HERS Performance-based Systems and Certification Programs}

There is a difference between the two commonly used systems for assessing the energy performance of housing, (1) performance-based systems and (2) certification programs. 
- Performance-based systems ${ }^{2}$ estimate and rate on a scale (usually from one to 100 ) the relative energy efficiency of any house, regardless of age, efficiency, or fuel use. Both new and existing houses can be rated. The rating is based on the thermal performance of the building envelope and equipment and is obtained by an on-site inspection (energy audit) and calculations. Performance-based systems estimate annual energy performance and costs, and may recommend cost-effective energy-efficiency improvements. Performance-based systems are often operated by state energy offices, municipal utilities, and nonprofit organizations.

- Certification programs, or pass/fail home energy-efficiency rating systems, are usually used for new homes, and are ordinarily operated by utility companies, home builders' organizations, or not-for-profit organizations. Energy-efficiency standards for these programs are developed using local building characteristics, construction practices, and climatic conditions. Certification programs, which are also based on analytical studies, usually include thermal envelope efficiency and space-conditioning efficiency criteria. These programs generally rely on a specified inspection/verification process to ensure rating consistency. Houses either pass or fail the inspection for energy efficiency.

\section{Purpose and Organization of the Report}

The purpose of this report is to portray and assess the nation's progress toward the widespread use of mortgages to finance the cost of energy improvements in housing. The report covers the work of the National Collaborative itself and assesses progress since the Collaborative reached its consensus agreements early in 1992.

The report is organized to trace major activities on EEMs and HERS since the A Blueprint for Action was released. The next section summarizes the activities of the National Collaborative, the distribution of the Blueprint, and comments on the Blueprint by the national-level stakeholder organizations involved in the Collaborative.

The section following covers the work of the HUD Task Force assembled under the National Affordable Housing Act (NAHA) of 1990, called the Section 946 Task Force. ${ }^{3}$ The Task Force was charged with developing a uniform plan for EEMs in the nation.

While the HUD Section 946 Task Force was continuing its work, the HERS industry and other stakeholders developed a HERS Council; its development and formation are the topics of the next section.

Congress passed legislation during 1992 that affects the use of EEMs and HERS during the next several years. The report's next section summarizes relevant sections of recent federal legislation. Several state legislatures have passed laws pertinent to HERS and EEMs and these are briefly mentioned.

Following are sections on federally mandated activities for HERS and pilot tests for EEMs. Other activities on the part of the states and of stakeholder groups in pursuit of either HERS, EEMs, or both, are then summarized. Finally, the conclusions section summarizes and assesses the progress made in the United States toward the widespread use of EEMs and HERS since the release of A Blueprint for Action in March 1992.

\footnotetext{
${ }^{2}$ Also frequently called "scaled" systems.

${ }^{3}$ Section 946 was the section of NAHA establishing the Task Force.
} 


\section{The National Collaborative and A Blueprint for Action}

Two events triggered attention on HERS and EEMs and focused action at the national level early in 1991. The first was passage of the National Affordable Housing Act of 1990 (P.L. 101-625), which directed HUD to develop a uniform plan to make housing more affordable through mortgage financing incentives for energy efficiency. The second was the release of the Bush administration's National Energy Strategy in February 1991, which set forth federal policy on the use of mortgage finance to increase housing efficiency. The policy called for the U.S. Department of Energy (DOE) and HUD to "develop and encourage the voluntary acceptance of efficiency ratings and their use in home financing" (U.S. Department of Energy 1991/1992, p. 11).

The Office of Building Technologies, Office of Energy Efficiency and Renewable Energy, DOE, in cooperation with HUD, initiated the National Collaborative on HERS and EEMs. The Collaborative's goal was to develop an action plan for the widespread promulgation of mortgage financing of energy improvements in housing linked with HERS. The National Collaborative involved members representing 25 organizations and interests that, working together, can make HERS and EEMs a national reality. The members represented states, the mortgage finance community, real estate professionals, builders, remodelers, public and environmental interests, utilities, and existing HERS programs.

The National Collaborative included four technical advisory committees in the subject areas of EEMs, HERS, implementation (on institutional issues), and awareness (on consumer disclosure, liability, labeling, marketing, and the like). The Collaborative worked on policy questions through a strict consensus process that was professionally facilitated. Each member present approved of the content of the Collaborative's report, $A$ Blueprint for Action. This document compiled the Collaborative's findings and recommendations regarding the steps to be taken to ensure the widespread use of EEMs linked with HERS. The text of $A$ Blueprint for Action is included as Appendix E to this report; it contains a list of Collaborative members.

The National Collaborative met 10 times between March 1991 and January 1992 and produced the consensus agreements contained in the Blueprint, which was released in March 1992. The National Collaborative included participants with sharply different perspectives on some issues. Yet in only one area-the EEMs program-members "agreed to disagree" on some of the provisions of a uniform approach to EEMs in the nation. Even here, there was a broad consensus on many actions that could be taken immediately.

A companion volume to the Blueprint was published which contained the issue papers prepared as part of the Collaborative process. This report, Going National with HERS and EEMs: The Collected Papers of the National Collaborative, presents some 52 one-page papers on technical issues.

\section{Dissemination and Distribution of the Collaborative's Reports}

The Collaborative's documents were widely disseminated. Member organizations received 25 copies apiece to distribute to their executives, staff members, and others within and beyond their organizations. The Collaborative's technical advisers and official observers also received copies, as did each state energy office. Many others requested copies. In all, several hundred copies of the Blueprint and of Going National were sent out, and requests for these documents were still being received more than a year later, in July 1993.

DOE issued a press release on the Collaborative, which triggered several press interviews. Articles appeared, for example, in the Washington Post, the Public Utilities Fortnightly, Nation's Building News, DOE This Month, Real Estate Today, The Solar Letter, and Professional Builder and Remodeler. 
In addition to coverage in the regular media, member organizations printed articles on HERS/EEMs and the Collaborative in the special trade association newsletters sent to their members.

Appendix D presents a partial listing of articles published on the National Collaborative, HERS, and EEMs from 1991 through 1993, based on the DOE/NREL archives. Many other relevant articles were undoubtedly published.

\section{Comments on A Blueprint for Action}

The Collaborative member organizations conducted reviews of the document and responded with formal comments. These comments were generally favorable toward the National Collaborative and toward DOE and HUD for initiating it, and supported the accuracy of the Blueprint in reflecting the Collaborative's consensus agreements, as well as its points of disagreement. A report, Organizational Review Processes, Comments, and Activities for the HERS/EEMs National Collaborative, dated September 30, 1992, documented organizational activities and comments (NREL 1992). Appendix C summarizes the organizational review comments on $A$ Blueprint for Action.

Although generally favorable comments were received, many member organizations said that major work still remained to be done before EEMs/HERS could be widely used. In many ways, the Blueprint was seen as a "to-do list" for forward progress in reaching the goal of making EEMs a consumer-useable reality.

An analysis of the organizational comments revealed a number of issues that the organizations identified as remaining to be resolved. The types of issues were (1) the nature of the linkage between HERS and EEMs, (2) EEMs issues, (3) HERS issues, (4) implementation issues, and (5) awareness issues.

\section{The nature of the linkage between HERS and EEMs}

The Collaborative had agreed with the desirability of a voluntary system and of stakeholder involvement in decision making. Participation should be voluntary, those commenting said, and stakeholders should be involved in formulating linkages between HERS programs and a uniform EEM process.

\section{EEMs issues}

- Determining borrower qualification. The Collaborative had stated that qualified applicants for standard mortgages should qualify for energy-efficient mortgages (p. 9). Commenters agreed that all potential borrowers should be equally eligible to participate.

- Including home improvement loans. The Collaborative had not had time to reach consensus on home improvement loans and had not made a recommendation on them. A reviewer commented that remodelers would become more involved in energy improvements if these loans were included. Further, remodeling may be a time when homeowners would be more willing to include efficiency improvements.

- Lack of data on default rates. The Collaborative had judged this research gap as a major barrier to the effort because it increased lender uncertainty about the risk of EEMs. Some reviewers agreed.

- Lender indemnification. The Collaborative recommended that lenders should be indemnified for any additional risk in EEMs. Comments varied in the perceived need for this. Some stated that lender risk would decrease with EEMs and others stated that, until lenders were more certain of the effects of EEMs on default rates, they should be indemnified from the risk they might be taking. 
- Property valuation. The valuation issue had not been solved in the Collaborative; it remained a key issue for the mortgage community because their mortgage investments need to be based on market response to property values. Such values can vary markedly, depending on local area market factors such as growth and recession.

\section{HERS issues}

- Accuracy/credibility of HERS systems. The Collaborative stated that HERS must produce consistent and accurate ratings to be credible. Commenters said that DOE and HUD should incorporate professional standards for ratings, scientifically valid measures, and full disclosure of consumer costs in any national HERS/EEMs guidelines. The accuracy of the HERS calculational tools was a key concern to the program-implementing organizations which commented.

- Quality control/certification. The Collaborative called for validation of HERS software and certification of HERS programs. Comments underscored the point, stating that certification of HERS programs should have a consistent meaning in the marketplace.

- Consensus standards. Some reviewers said the relationship between building standards and EEMs needed clarification. The Collaborative had not dealt with this issue.

- Fuel neutrality. The Collaborative deferred fuel neutrality to local jurisdictions. Commenters said that information on projected energy costs should be provided to consumers.

- HERS measurements. The Collaborative decided that such measures as shading and awnings, which reduce solar gain, should be included as part of HERS thermal envelope measurements. Some commenters reiterated the importance of including such measures. Others wanted energy management systems to be included in HERS measurements.

- Environment, health, and safety issues. The Collaborative was silent on these issues. Comments suggested that guidelines should be developed to ensure that energy-efficient homes are durable, healthy, sustainable, and provide a quality living environment.

- Rating scale. The Collaborative stopped short of recommending a uniform rating scale, although it said that "a single rating method should be the basis for linking HERS and EEMs." Some organizations observed that the issue between rating and certification remained unresolved, as did the issue of whether there should be a single rating method. The Collaborative did agree, however, that any rating should be expressed as total annual energy use in Btu per year by fuel type.

\section{Implementation issues}

- Data base. The Collaborative strongly recommended establishment of a national data base. Comments were that the expense of setting up a national data base should be considered.

- Need for public subsidies. Among commenters, there was some disagreement about the extent to which the federal government should subsidize the development of the nationwide EEMs/HERS program. The Collaborative had not addressed this issue.

- Organizational roles. The Collaborative failed to agree on, designate, or assign roles and responsibilities for carrying out a national program. Some reviewers called for identification and recommendation of the key players and said they wanted a national forum to continue discussions on the implementation issues until these were resolved. 
- Pilot testing. Demonstration programs had been recommended by the Collaborative. Pilot tests of HERS/EEMs concepts and protocols were viewed as necessary by reviewers.

\section{Awareness issues}

- Disclosure. The Collaborative wanted to raise consumer awareness on the use of EEMs and HERS. It found that most consumers have never heard of EEMs and HERS. Real estate professionals commented that they did not want to be liable in connection with disclosures, while other commenters noted that real estate professionals should play a significant role in disclosure.

- Training. Echoing the Collaborative's findings, commenters perceived a critical need for training of raters, builders, lenders, real estate professionals, utilities, and consumers for the program to succeed.

The National Collaborative resolved some issues and defined additional areas of concern. Activities since publication of the Blueprint aimed at increasing the use of HERS and EEMs are assessed in this report. These actions constitute ongoing efforts to resolve the remaining issues.

\section{Future of Collaborative Efforts}

By September 1993, DOE had decided not to reconvene the National Collaborative, which, in any event, had been an ad hoc task force from the outset. DOE judged that sufficient activity was occurring in the HERS/EEMs arena nationwide, and that the Collaborative would not have further utility as a venue for resolving remaining issues in the near term. In lieu of the Collaborative, two groups have formed that appear ready to carry on the effort. The HUD Section 946 Task Force (described in the next section) may evolve into an ongoing group for the members of the mortgage community concerned with energy efficiency in lending. The HERS Council, developed by the Collaborative membership, is described in the following section. The Council is working to resolve HERS issues and deal with how HERS might link with EEMs and other programs. The door remains open for a future collaborative if and when federal agencies, federally chartered financial institutions, and the stakeholder groups involved believe it would be of value. 


\section{The HUD Section 946 Task Force}

The National Affordable Housing Act of 1990 (P.L. 101-625), Section 946, required the Secretary of HUD, in consultation with the Secretary of DOE, to "promulgate a uniform plan to make housing more affordable through mortgage financing incentives for energy efficiency." The law specified that a plan for EEMs should be promulgated no later than two years after the date of enactment, in November 1992.

To develop the plan, HUD's Office of Insured Single Family Housing, Office of the Assistant Secretary for Housing, assembled the HUD/FHA Task Force on June 11, 1992 "to make recommendations on financing energy efficiency in private mortgages" (HUD 1992). Task Force members, as required by the 1990 Act, included representatives from DOE, HUD/FHA, Freddie Mac, and Fannie Mae. In addition, HUD invited several others to serve as Task Force resources, including representatives from the Mortgage Bankers Association (MBA), the National Association of Home Builders (NAHB), the National Association of REALTORS (NAR), the Government National Mortgage Association (Ginnie Mae), the Alliance to Save Energy (ASE), the Edison Electric Institute (EEI), and Energy Rated Homes of America (ERHA).

The Task Force met in June, September, and November 1992. Its five objectives, prepared by HUD in accordance with the affordable housing mandate, were to:

1. Develop standards for approval of HERS, including certification procedures and quality controls

2. Develop common core standards for EEMs among the agencies

3. Remove unnecessary barriers to EEMs

4. Develop a national data base on the characteristics of EEMs, including default information

5. Develop public awareness strategies.

The Task Force created teams to research and write resource papers on the following issues.

- Energy audits

- HERS

- Certification programs

- Codes/standards/guidelines

- Issues of cost-effectiveness and affordability

- Appraisals and the value of energy efficiency

- $\quad$ EEM concepts

- New and existing housing

- Other housing technical issues.

HUD/FHA drafted several alternative plans for EEM pilot programs for Task Force consideration. Five options were considered. Task Force members discussed the problem of how to recognize the value of energy improvements in the appraisal process and how to establish borrower qualifying ratios by using PITI + E (or energy cost) and other variations of that formula. The Task Force did not reach consensus on any of the proposals.

On February 18, 1993, HUD issued a draft report, "Uniform Plan of Action for Energy Efficient Mortgages" for review by Task Force members. A modified report summarizing the Task Force's recommendations was submitted to the Housing Commissioner for signature in June 1993. Commissioner action on the report was still pending as of September 14, 1993.

HUD's intention is to develop a user-friendly EEMs program with common standards and procedures. The HUD Task Force may recommend that four EEM program options and the five-state EEM pilot 
program should serve as the basis for the uniform plan required by the National Affordable Housing Act. If so, this means that a final uniform plan might not be formulated until the results of the pilot programs are in hand in approximately the fall of 1995. 


\section{The Home Energy Rating System Council}

\section{Introduction and Background}

The HERS Council grew out of the National Collaborative. A month after the release of $A$ Blueprint for Action, a limited group of HERS providers and representatives from other energy organizations met on April 15, 1992, in San Francisco to discuss how the momentum generated by the Collaborative might best be continued. The group envisioned the formation of a permanent, national organization to

- Further develop and improve uniform HERS guidelines

- Provide a link with EEMs programs

- Educate and communicate with the public about HERS/EEMs.

This initial group formed the HERS Council Planning Committee to oversee the establishment of such an organization. In September 1992, committee members secured DOE support and the services of D \& R International, Ltd. as its administrative agent. DOE reaffirmed its commitment to work with the HERS Council and urged the council to work in partnership with it on those portions of the Energy Policy Act-signed into law by President Bush on October 18, 1992-requiring DOE to develop uniform, voluntary guidelines for HERS.

\section{Planning Activities}

On October 29, 1992, the Planning Committee convened a meeting of potential council members in Washington, D.C. Representatives from more than 50 organizations attended. The Planning Committee provided an overview of the April 1992 meeting and developments since that time. Attendees discussed the information presented and formed three working groups to develop recommendations on the HERS council's mission and goals, its bylaws and articles of incorporation, and its technical agenda. The Planning Committee met on December 10, 1992, to refine the council's mission, goals, board composition, and proposed bylaws, and to discuss a spring 1993 meeting of the new organization's members. At the October meeting, the Planning Committee-initially composed of members from the HERS industry (utilities and state and local providers)-broadened its membership to include representatives from state and local governments without HERS programs, energy-efficiency advocates, environmental and consumer groups, real estate, and financial and building industries-a profile that more closely reflected the diverse membership of the National Collaborative.

At the December 1992 meeting, the Planning Committee unanimously adopted a preliminary mission statement for the HERS Council:

The mission of the Home Energy Rating Systems Council is to increase energy efficiency in housing by serving as an education and research resource and by establishing a voluntary program for accrediting systems and certifying tools that assign home energyefficiency ratings, consistent with uniform guidelines to access energy-efficient mortgages and other programs.

The Planning Committee also reached consensus on the following goals for the HERS Council.

\section{Immediate actions}

- Establish minimum, uniform guidelines for certifying home energy-efficiency rating tools

- Establish minimum, uniform guidelines for accrediting home energy-efficiency rating systems

- Establish accreditation and certification procedures 
- Develop and strengthen linkages to EEM and other programs

- Develop protocols that encourage uniform application of home energy ratings

- For evaluation purposes, develop procedures for uniform data collection.

\section{Implementation goals}

- Contribute to research agendas that address common issues of importance to council members

- Serve as a communication and education resource for members and others

- Upon request, evaluate and certify home energy rating tools; re-evaluate on a periodic basis

- Upon request, evaluate, accredit, and inspect home energy rating systems; re-accredit and inspect on a periodic basis

- Provide for data collection and dissemination.

\section{Ongoing goals}

- Establish and enhance linkages with other programs to utilize information produced by HERS

- Promote the use of home energy ratings as a rational basis for home energy decisions

- Enhance communication, information sharing, and coordination among council members and others.

The Planning Committee adopted "Home Energy Rating Systems Council" as the organization's formal name. The Bylaws and Articles Working Group-a subgroup of those who had participated in the October 29, 1992, meeting-developed draft bylaws for the council. These bylaws were later revised to meet state statutes and were adopted when the council was incorporated on February 17, 1993.

\section{Membership}

The Planning Committee also reached agreement on the council's membership and the Board.of Directors at the December 1992 meeting. Considering recommendations presented by the Articles and Bylaws Working Group, the Planning Committee agreed that potential members of the HERS Council should include

- HERS providers

- Utilities

- State and local governments

- Financial community

- Building industry

- Consumer affairs
- Energy and environmental interests

- Real estate professionals

- Appraisers

- Codes/standards bodies

- Home inspectors

- HERS technical/support contractors.

This membership structure provided broad representation of those who would need to be involved in implementing HERS programs.

\section{Representation on the Board of Directors}

The Planning Committee, in addition, agreed upon a structure for the council's Board of Directors. The profile of the 19 board members follows:

- HERS providers: scaled programs (2), certified programs (2) 
- Utilities: electric association (1), gas association (1)

- State/local government (2)

- Financial community: primary lenders (1), secondary lenders (1)

- Building industry: new building association (1), retrofit association (1)

- Manufacturers: product (1), equipment (1)

- Consumer groups (1)

- Energy/environment groups (1)

- Real estate agencies (1)

- Appraisers (1)

- At large (not from above groups) (1).

At the December 1992 meeting, the Planning Committee resolved to incorporate in a jurisdiction that provided maximum protection for limitation of liability of directors and officers. Based on research into the relevant sections of state code in several states, the Planning Committee unanimously selected Maryland, and the council was formally incorporated there on February 17, 1993.

\section{March 1993 Organizing Meeting}

The agenda for the March 15, 1993, HERS Council Organizing Meeting included discussion of the bylaws, election of the Board of Directors, and discussion of the technical and policy issues related to developing national, voluntary HERS guidelines. The 1992 Energy Policy Act (EPAct) requires DOE to promulgate guidelines by April 1994; guidelines must be developed by October 1993 if they are to receive the various clearances required by the April 1994 deadline.

The mission statement and the goals endorsed by the Planning Committee were embodied in the organization's bylaws when it was incorporated. Attendees at the HERS Council organizing meeting on March 15, 1993, reviewed and discussed the mission and goals. Because the HERS Council was incorporated prior to the meeting, only the Board of Directors could modify the bylaws. Meeting attendees made several recommendations for the modification of the bylaws to the Board of Directors.

\section{Board of Directors and Officers}

A nominating committee developed a slate of candidates for the Board of Directors for consideration by the Council's members. Directors elected at the March 1993 meeting were as follows:

\section{HERS providers (4 seats)}

Scaled programs ( 2 seats)

David Altscher, CA Home Energy Efficiency Rating System (CHEERS, Inc.)

Ron Hughes, Energy Rated Homes of America

Certified programs ( 2 seats)

Benny Folsom, Good Cents

Thomas Rooney, Energy Crafted Homes

Appraisers (1 seat): Donald Kelly, Appraisal Institute 


\section{Building industry (2 seats)}

New building association (1 seat): Ron Burton, National Association of Home Builders

Existing buildings association ( 1 seat): Representative pending

Consumer groups (1 seat): Rebecca Cohen, Consumer Federation of America

Energy and environmental groups (1 seat): Malcolm Verdict, Alliance to Save Energy

Financial community (2 seats)

Primary lenders (1 seat): Bruce Bowler, Mortgage Bankers Association

Secondary lenders (1 seat): Chip Coffay, Federal National Mortgage Association (Fannie Mae)

\section{Manufacturers (2 seats)}

Equipment (1 seat): Linda Whitman, Honeywell, Inc.

Product (1 seat): Dwight Shuler, Owens-Corning Fiberglas

Real estate industry (1 seat): Stephen O'Connor, National Association of REALTORS

\section{State and local government (2 seats)}

Deborah Howard, National Association of State Energy Officials (Arizona State Energy Office)

Allan Hunt, National Council of State Housing Finance Agencies

\section{Utilities (2 seats)}

Electric association (1 seat): Tom Farkas, Edison Electric Institute

Gas association (1 seat): Mary Carson, American Gas Association

\section{At-large member (1 seat): Walter Patterson, Texas Energy Extension Service.}

The board, meeting both on March 15 and 16, 1993, established an Executive Committee composed of the following officers:

- Deborah Howard, National Association of State Energy Officials, Chair

- Malcolm Verdict, Alliance to Save Energy, Vice Chair

- Ron Burton, National Association of Home Builders, Secretary

- Tom Farkas, Edison Electric Institute, Treasurer

- Benny Folsom, Southern Electric International

- Ron Hughes, Energy Rated Homes of America

- Allan Hunt, National Council of State Housing Finance Agencies.

\section{Standing Committees}

The participants at the March 1993 organizing meeting recommended to the board that the bylaws be amended to provide for standing committees. Meeting attendees also recommended that membership on committees be limited to members that had applied to, and had been approved by, the board. They also suggested that committees should have a limited number of members and that appointments to committees 
should be influenced to provide a balance of interests on the committees. Regular attendance of the committee members was also recommended.

In addition, the meeting attendees made the following recommendations about committee operations to the Board of Directors:

- Committee chairs should be designated by the Board of Directors

- Committee chairs should be accountable to the Board of Directors

- The composition and balance of committees should be determined by the committee chairs, with board oversight; the committee chairs should work with the board to designate members and subcommittee members

- Committee votes should be by majority rather than by consensus

- Committees should make "output" available to committee nonmembers on request, and all comments should be acknowledged.

In its subsequent deliberations, the board established three standing committees:

- Technical Committee

- Education Committee

- HERS/EEMs Linkage Committee.

The board accepted applications from members for positions on the standing committees, and developed operating procedures for the committee, taking into account the comments of the members.

The chairs of the committees were appointed in May 1993 and are as follows:

Technical Committee:

Dick Bahn, Honeywell, Chair

Richard Tracey, Ryland Homes, Co-chair

Education Committee:

Chris Taylor, ERHV, Chair

Patty Donaldson, Honeywell, Co-chair

HERS/EEMs Linkage Committee: Cindy Pentecost, Salt River Project (AZ), Chair

Doug Lothrop, Vermont Housing Finance Agency, Co-chair.

\section{Continuing Activities}

At its June 4, 1993, meeting in Washington, D.C., the board began work on a strategic plan for the Council and also dealt with several administrative issues. The next board meeting is planned for October 1994, in Atlanta. As of September 9, 1993, the HERS Council had 128 members. 


\section{Legislation in Support of HERS and EEMs}

In October 1992, President Bush signed three bills containing regulations that promote HERS and EEMs: ${ }^{4}$

- The National Energy Policy Act of 1992 (EPAct) amends sections of the Energy Conservation and Production Act (P.L. 94-385), the Energy Conservation Policy Act (P.L. 101-218), and the CranstonGonzalez National Affordable Housing Act (P.L. 101-625). EPAct directs DOE to develop and issue voluntary HERS guidelines. EPAct also requires federally assisted housing to comply with residential efficiency building standards to be established by HUD and the U.S. Department of Agriculture (USDA) by October 1993.

- The Housing and Community Development Act of 1992 (P.L.102-550) contains provisions identical to those in EPAct that require HUD to implement an EEMs pilot program for existing housing. The five-state pilot program will precede a Secretarial recommendation in 2 years on the feasibility of a uniform nationwide EEM program.

- The Veterans Home Loan Program Amendments of 1992 (P.L. 102-547) mandate a 3-year program to demonstrate the feasibility of EEMs that take energy efficiency into account in veterans' loans for existing housing.

These three bills set forth provisions in five different, but related, areas:

1. Residential energy-efficiency rating guidelines

2. HUD/FHA EEMs pilot tests in five states

3. A 50-state DVA EEMs demonstration for veterans

4. Expansion of HUD Section 946 Task Force membership

5. Mandating HUD and FmHA use of more energy-efficient building standards for new housing mortgages.

\section{Residential Energy-Efficiency Rating Guidelines}

EPAct (Title I, Sec. 102) requires that, by spring 1994, DOE, in consultation with HUD, DVA, HERS providers, and other appropriate persons, promulgate voluntary guidelines that encourage uniformity in HERS. The voluntary guidelines may be used by state and local governments, utilities, builders, real estate agents, lenders, agencies in mortgage markets, and others. The bill stipulates that a national, voluntary HERS program must meet the conditions that follow.

1. Encourage uniformity with regard to systems for rating the annual energy efficiency of residential buildings

2. Establish protocols and procedures for

- Certification of the technical accuracy of building energy analysis tools used to determine energy-efficiency ratings

- Training of personnel conducting energy-efficiency ratings

\footnotetext{
${ }^{4}$ President Bush signed the National Energy Policy Act on October 24, 1992. He signed the Housing and Community Development Act and the Veterans Home Loan Program amendments on October 28, 1992.
} 
- Data collection and reporting

- Quality control

- Monitoring and evaluation.

3. Encourage consistency with, and support for, the uniform plan for federal energy mortgages being developed by the HUD Section 946 Task Force

4. Provide that rating systems take into account local climate conditions and construction practices, solar energy collected on-site, and the benefits of peak load shifting construction practices, while not discriminating among fuel types, and

5. Establish procedures to ensure that residential buildings can receive an energy-efficiency rating at the time of sale and that such a rating is communicated to potential buyers.

Under EPAct, DOE must by October 1994 establish a technical assistance program consistent with national guidelines to facilitate the use of HERS by states and localities. By October 1995, DOE must submit a report to the President and Congress describing the implementation of the HERS guidelines, as well as recommendations on the feasibility of requiring energy-efficiency ratings as a prerequisite to receiving federally assisted, guaranteed, or insured mortgages. The report must also cover the actions of other federal agencies, the states, local governments, and other organizations to implement the voluntary guidelines and any problems encountered in implementing them.

\section{HUD/FHA EEMs Pilot Tests in Five States}

EPAct (Title I, Sec. 106) and the Housing and Community Development Act of 1992 (Title V, Sec. 513), using identical language, require HUD to establish an EEMs pilot program in five states by April 1993. The purpose of the program is ". . . to promote the purchase of existing energy-efficient residential buildings and the installation of cost-effective improvements in existing residential buildings."

HUD/FHA will implement the pilot program. Individuals residing in any of the five pilot states, with approved income and credit records, are eligible to apply for an EEM under the program. The legislation stipulates that the cost of energy-efficient improvements may not exceed the greater of (1) $5 \%$ of the property value (not to exceed $\$ 8,000$ ) or $(2) \$ 4,000$.

The legislation allows lenders to

- Permit final loan amount to exceed the loan limits established by the National Housing Act by an amount not to exceed $100 \%$ of the cost of the cost-effective energy-efficiency improvements

- Hold in escrow all funds provided to the mortgagor to undertake the energy-efficiency improvements until the efficiency improvements are actually installed

- Transfer or sell the EEM to the appropriate secondary market agency, after the mortgage is issued; this may occur before the efficiency improvements are actually made. 
HUD will promote EEMs in the pilot states by

- Providing information to lenders and others on the availability and advantages of EEMs

- Requiring lenders to provide written notice of the availability and benefits of the pilot program to individuals applying for mortgages in participating states

- Requiring each applicant for a mortgage insured under the National Housing Act in pilot program states to sign a statement attesting that the applicant has been informed of the program requirements and understands the benefits of EEMs.

The legislation requires HUD to develop and implement, by July 1993, a training program for personnel at lending agencies, real estate companies, and other appropriate organizations regarding the benefit of EEMs and the operation of the pilot program. By April 1994, HUD must submit a report to Congress describing the implementation and effectiveness of the EEMs pilot program and evaluating the potential for expanding the program nationwide. The legislation mandates a nationwide expansion of the EEMs program to begin no later than October 1994 , contingent upon a favorable assessment of the five-state pilot projects. The national program will authorize EEMs for both existing and new housing.

\section{A 50-State DVA EEMs Demonstration for Veterans}

The Veterans Home Loan Amendments (Title 38, Sec. 9) require the DVA to implement a nationwide program demonstrating the feasibility of guaranteeing loans for acquiring and making energy-efficiency improvements to an existing home or for making energy-efficiency improvements to a home owned and occupied by a veteran. The amendments expand DVA loan eligibility to include reservists who have served for at least six years.

DVA loans for energy-efficiency improvements may not exceed the greater of (1) the cost of the improvements up to $\$ 3,000$, or (2) $\$ 6,000$, if the increase in the monthly payment does not exceed the likely reduction in monthly utility costs resulting from the energy-efficiency improvements. The legislation requires the DVA to guarantee loans for an amount equal to the cost of the house plus the cost of the energy-efficiency improvements.

Energy-efficiency improvements included in the Act's provisions are

- Solar heating and cooling systems, including passive systems (windows and skylight glazing; thermal floors, walls, and roofs; movable insulation panels; solar furnaces; double-pane windows; and other features)

- Caulking and weatherstripping

- Furnace efficiency modifications

- Clock thermostats

- Ceiling, attic, wall, and floor insulation

- Water heater insulation 
- Storm windows and doors

- Heat pumps.

The legislation authorizes the DVA to guarantee EEMs through December 31, 1995. The DVA must submit yearly reports for the duration of the demonstration program to the Committees on Veterans Affairs of the Senate and House of Representatives. The reports will contain information pertaining to

- The number of EEMs guaranteed by the DVA

- The average amount of money added to the mortgage to finance energy-efficiency features

- The types of energy-efficiency features obtained with DVA EEMs

- The default rates on EEMs guaranteed by the DVA compared with the default rates on all other types of mortgages guaranteed by the Secretary.

\section{Expansion of HUD Section 946 Task Force Membership}

EPAct (Title I, Sec. 105) bolsters Section 946 of the Cranston-Gonzalez National Affordable Housing Act requiring HUD, in consultation with DOE, to promulgate a uniform mortgage financing plan for energy efficiency. It amends the legislation by explicitly defining EEMs, and broadening the membership and mandate of the HUD Task Force.

Sec. 105 of EPAct defines an EEM as

... a mortgage that provides financing incentives for the purchase of energy efficient homes, or that provides financing incentives to make energy-efficiency improvements in existing homes by incorporating the cost of such improvements in the mortgage.

The legislation removes limitations on HUD Task Force membership, which had been restricted to representatives from federal housing mortgage programs at HUD, FmHA, mortgage loan and insurance programs of the USDA, Fannie Mae, and Freddie Mac. In addition, EPAct requires the HUD Task Force to determine the value of notifying potential home purchasers of the availability of EEMs to promote energy efficiency in residential buildings.

Sec. 105 amends the language of the Cranston-Gonzalez National Affordable Housing Act to state that the membership should include, "but not be limited to," FHA, FmHA, FNMA, and FHLMC. This language suggests that the Task Force membership could be broadened to include other stakeholder groups with whom the Task Force had been mandated to consult-mortgage bankers, home builders, real estate brokers, private mortgage insurers, energy suppliers, and nonprofit housing and energy organizations.

EPAct also requires the Task Force ". . . to determine whether notifying potential home purchasers of the availability of energy efficient mortgages would promote energy efficiency in residential buildings, and if so, ..." to recommend notification guidelines. 


\section{Mandating HUD and FmHA Use of More Energy-Efficient Building Standards for New Housing Mortgages}

EPAct (Title I, Sec. 101) requires HUD and USDA to jointly establish and promulgate energy-efficiency standards for

- New construction of residential housing (public and assisted housing and single-family and multifamily residential housing) subject to mortgages insured under the National Housing Act

- New construction of single-family housing subject to mortgages insured, guaranteed, or made by USDA under Title V of the Housing Act of 1949.

The standards must meet or exceed the requirements of the Council of American Building Officials Model Energy Code, 1992 (CABO-MEC 92) for new single-family or low-rise multifamily housing. For multifamily high-rise buildings, the standards must meet or exceed the requirements established by the American Society of Heating, Refrigerating, and Air-Conditioning Engineers (ASHRAE Standard 90.1-1989).

The legislation directs HUD and USDA to establish energy-efficiency standards by October 1993. An advisory Task Force composed of homebuilders; national, state, and local housing agencies (including public housing agencies); energy agencies; building code organizations and agencies; energy-efficiency organizations; low-income housing organizations; and other parties designated by HUD and USDA will serve as a resource as the agencies develop energy-efficiency criteria for new housing construction.

If either CABO-MEC 92 or ASHRAE Standard 90.1-1989 are revised, HUD and USDA must amend their standards to either meet or exceed the requirements of the revised code or standard. EPAct exempts HUD and USDA from amending their standards if the Secretaries determine that compliance with the new code or standard would not result in significant energy savings, or would not be technologically feasible or economically justified.

\section{State Legislative Activity in Support of HERS and EEMs}

Between 1990 and 1993, one state, Florida, passed legislation requiring that the prospective purchaser of a single or multifamily dwelling receive written notification that the building's energy efficiency may be determined prior to or at the time of real estate transactions. The legislation mandates that the energyefficiency rating of a new residential or commercial building must be disclosed in writing prior to or at the time of purchase.

Four other states enacted legislation requiring the development and implementation of voluntary HERS programs. These were:

- California

- Connecticut

- Oregon

- Iowa.

Six states passed legislation on EEMs. The Alaska legislature assigned responsibility for a HERS program linked with EEMs to the Alaska Housing Finance Corporation (AHFC). Montana legislation appropriated $\$ 300,000$ in petroleum violation escrow (PVE) funds to establish an energy-efficiency mortgage reserve account that would enable the Board of Housing to sell bonds and offer loans for energy-efficient new homes costing more than the FHA limit. Iowa and Florida legislated to develop and make available 
brochures for home purchasers on home energy-efficiency ratings and on the possibility that a rating may qualify a purchaser for an EEM. Legislation enacted in Arkansas directs the state energy office to develop and implement a pilot program to increase the use of EEMs in the state. The legislation requires that the pilot meet the eligibility requirements of the HUD/FHA EEMs pilot program specified by the Energy Policy Act of 1992. New Jersey passed a resolution endorsing the state's involvement in the HUD/FHA EEMs pilot program, should the state be one of the designated participants.

Legislation introduced in Louisiana and Massachusetts pertaining to HERS and EEMs failed to pass. 


\section{Residential Energy Efficiency Rating Guidelines}

As discussed in the section on legislation, EPAct requires DOE to promulgate, by rule, voluntary guidelines to encourage uniformity in HERS in consultation with other agencies, HERS providers, and others. DOE has decided to work in partnership with the Technical Committee of the HERS Council to develop the minimum guidelines for HERS programs. Table 1 summarizes National Collaborative recommendations and the related EPAct requirements. The EPAct requirements for HERS programs are substantially similar to the National Collaborative recommendations. This is not surprising, because some members of the Collaborative are known to have influenced the shape of national energy legislation.

Table 1. Comparison of National Collaborative Recommendations and
EPAct Requirements on Residential Energy Efficiency Ratings

\begin{tabular}{|c|c|}
\hline National Collaborative Recommendations & EPAct Requirements \\
\hline $\begin{array}{l}\text { Technically credible, voluntary minimum } \\
\text { guidelines that promote uniformity among local } \\
\text { systems (pp. 16-18) and apply to single-family, } \\
\text { multifamily, and manufactured housing } \\
\text { (p. 17). }\end{array}$ & $\begin{array}{l}\text { Voluntary guidelines shall encourage } \\
\text { uniformity for rating the annual energy } \\
\text { efficiency of residential buildings } \\
\text { (Sec. } 271[\text { b][1]). }\end{array}$ \\
\hline $\begin{array}{l}\text { To be accredited, HERS programs should } \\
\text { include } \\
\text { - Technically accurate software tool } \\
\text { - Rater training } \\
\text { - Data collection } \\
\text { - Quality control (pp. 16-18). }\end{array}$ & $\begin{array}{l}\text { Establish protocols and procedures for } \\
\text { - Tool certification } \\
\text { - Rater training } \\
\text { - Data collection and reporting } \\
\text { - Quality control } \\
\text { - Monitoring and evaluation. }\end{array}$ \\
\hline $\begin{array}{l}\text { Rating systems should account for } \\
\text { - Building type and climate zone } \\
\text { - Innovative designs and technologies } \\
\text { - Occupant behavior through standardized } \\
\text { assumptions } \\
\text { - Fuel neutrality (pp. 16-18). }\end{array}$ & $\begin{array}{l}\text { Rating systems must account for } \\
\text { - Local climate conditions and construction } \\
\text { practices } \\
\text { - Solar energy collected on-site } \\
\text { - Benefits of peak-load shifting } \\
\text { - Fuel neutrality. }\end{array}$ \\
\hline Raise public awareness at time of sale (p. 9). & $\begin{array}{l}\text { Time of sale rating and communication to } \\
\text { potential buyers. }\end{array}$ \\
\hline Linkage of HERS and EEMs (pp. 6-7). & $\begin{array}{l}\text { Recommendations on feasibility of linkage } \\
\text { with federally assisted, guaranteed, or insured } \\
\text { mortgages. }\end{array}$ \\
\hline
\end{tabular}

apage references are to A Blueprint for Action. 
The work of the Collaborative's Technical Advisory Committee will be a starting point for the development of the HERS calculation tool verification and minimum program guidelines. The collaborative recommended that software tools should:

- Use Btu/year by fuel type as the basic unit of measure

- Include both new and existing housing in energy use calculations

- Address energy use associated with

- Thermal envelope

- Mechanical heating, ventilating, and air conditioning (HVAC) system

- Fixed or movable shading elements 5

- Hot water service

- Optional features, such as hard-wired lights, major appliances and controls, and energy-storage systems.

The involvement of National Collaborative members in the HERS Council will help to ensure continuity in the technical work. It will also mean that those who are implementing HERS programs will be devising guidelines that should be practical, effective, accurate, and user friendly.

Depending on the availability of sufficient resources, DOE, with the participation of the HERS Council and NREL, is proposing to develop the HERS guidelines on the following schedule:

\section{Planned Completion Date}

March 1994

\section{Milestone}

Alternative rating approaches

Fuel neutrality

New vs. existing homes

Features

Output

Training

Labeling

Group ratings

Re-rating

Linkage to codes

Certification

Monitoring and evaluation

Guidelines document

Software test method

December 1994

Monitoring and evaluation protocols on technical matters

March 1995

Data archive and analysis

By October 1994, DOE is required to establish a program that will provide technical assistance to state and local organizations to encourage the use of ratings. And, in October 1995, DOE will report to Congress on federal action to implement the policies; actions by states, local governments, and other organizations to implement the guidelines and resolve any problems experienced; and recommendations on whether a HERS should be required to receive a federally assisted, guaranteed, or insured mortgage.

${ }^{5}$ E.g., awnings, wing walls, overhangs, eaves, and shade screens. 


\section{Pilot Tests for Energy-Efficient Mortgages}

Several different pilot tests for EEMs are being started. This section briefly describes the recently announced HUD/FHA EEMs pilot in five states; the DVA 50-state 3-year EEMs demonstration program; Fannie Mae EEMs pilots; and the Environmental Protection Agency's (EPA) pilot in Wisconsin.

\section{HUD/FHA 5-State Pilot}

As noted, the National Energy Policy Act of 1992 (Title I, Sec. 106) and the Housing and Community Development Act of 1992 (Title V, Sec. 513) direct HUD to establish an energy-efficient mortgage pilot program in five states. The Federal Housing Administration (FHA) developed the guidelines for the program, for which it has no separate appropriation.

The program has the following features:

- At least five states are being included in the pilot. The states selected have HERS programs already in place.

- The program is being implemented through a formal "Mortgagee Letter" that will inform lenders in the pilot states to provide EEMs. Lenders will be responsible for program implementation.

- Fannie Mae first approved the HERS programs in states selected by HUD before they were included in the pilot.

In deciding which five states would participate in the pilot tests, HUD/FHA invited material from states on their activities.

HUD announced the EEMs pilot tests to lenders through a mortgagee letter dated May 24, 1993 (Mortgagee Letter 93-13). The letter, from the Assistant Secretary for Housing/Federal Housing Commissioner Nicolas P. Retsinas, addressed some 12,000 lenders on "Single Family Loan ProductionEnergy-Efficient Mortgage Pilot Program." It stated:

In compliance with Section 513 of the Housing and Community Development Act of 1992, HUD is establishing an FHA Energy-Efficient Mortgage (EEM) Pilot Program for existing properties located in the following states: Alaska, Arkansas, California, Vermont and Virginia. This Pilot Program is effective immediately (Mortgagee Letter 93-13, p. 1).

The HUD EEMs pilots are unique in the following ways:

1. If the energy improvements pay for themselves through lowered utility bills, then $100 \%$ of their cost can be added to the amount of an FHA mortgage without an appraisal.

2. The EEM can exceed traditional loan limits by the amount of the improvement.

3. EEMs will be available to people who want to refinance their properties.

4. The program provides that up to $\$ 200$ of the cost of a HERS audit can be financed as part of the EEM. 
5. Every borrower for an FHA-insured mortgage will receive a disclosure statement describing EEMs, their benefits, and how to apply for one.

The effectiveness of the pilot depends in part on information and training. HUD/FHA added training in EEMs to its FHA field staff training beginning in March 1993. Lender training is scheduled in California in June 1993, and in Virginia, Alaska, and Vermont in August 1993.

At the end of an 18-month period, the Secretary of HUD is required to submit a report to the Congress describing the effectiveness and implementation of the EEMs pilot program and assessing the potential for expanding it nationwide.

HUD/FHA also intends to pilot test EEMs options recommended by the HUD Section 946 Task Force when these are announced.

\section{DVA's 50-State Pilot}

As described in the section on legislation, the Veterans Home Loan Program Amendments of 1992 require DVA to implement a program demonstrating the feasibility of EEMs for homes owned and occupied by veterans and reservists. The DVA will guarantee loans for an amount equal to the cost of the house plus the cost of efficiency improvements up to $\$ 3,000$, or cost-effective improvements up to $\$ 6,000$, if the increase in the monthly payment does not exceed the likely utility cost savings. The Department will report annually to Congress on the demonstration program, which is scheduled to run through December 31, 1995.

\section{Fannie Mae EEMs Pilots}

Since the National Collaborative released the Blueprint, Fannie Mae has been active in developing partnerships in several states to pilot test EEMs concepts. Pilots in three states have been established so far: Maryland, Vermont, and Washington.

\section{Maryland}

In January 1993, the Southern Maryland Electric Cooperative (SMECO) began working with Fannie Mae's Atlanta office to develop an innovative DSM program-called the PowerSaver Home Program-linked with EEMs. SMECO offers rebates of up to $\$ 3,500$ to purchasers of new electric homes. Fannie Mae will allow purchasers to apply the utility rebate to closing costs, downpayment, or reserve funds.

To be eligible for PowerSaver Home rebates, new homes must be built by participating builders who meet the following requirements. Builders must

- Sign an agreement stating they will abide by SMECO's energy-efficiency construction standards

- Register all new homes prior to laying the foundation. SMECO will review the plans to make sure the utility's standards are being met. SMECO employees will conduct on-site construction inspections, including an inspection of the completed home

- Use a heating contractor who is participating in SMECO's rebate program. 
SMECO is taking an active role in training lenders, real estate agents, builders, and heating contractors. In February and March 1993, the utility sponsored a seminar for builders.

As of April 1, 1993, 43 homes were registered in the PowerSaver Home Program.

\section{Vermont}

Early in 1992, the Vermont Housing Finance Agency (VHFA) approached Fannie Mae with a proposal for a Vermont EEMs pilot project. Fannie Mae responded with an offer of $\$ 20$ million in secondary mortgage financing for EEMs in Vermont with VHFA acting on behalf of Vermont lenders. Under the agreement, Fannie Mae will back $\$ 20$ million in EEMs for approximately 250 loans. Historically, the VHFA has worked with Vermont lenders who sold mortgages to them. A limited number of lending institutions will originate EEMs and sell them to Fannie Mae directly.

The agreement permits qualified borrowers or refinancers to include up to $\$ 5,000$ in energy-efficiency improvements in their mortgages with no additional appraisal or underwriting once the base loan is approved, providing that the value of the energy savings is documented as cost-effective by Energy Rated Homes of Vermont (ERH of VT) by using a modified Form 70A value calculation.

The VHFA, with the support of Fannie Mae, ERH of VT, and the lenders, originally attempted to raise $\$ 20$ million in below-market-rate investment capital from socially responsible investment firms and foundations to finance EEMs. This attempt was unsuccessful. In January 1993, it was decided to market EEMs at the standard interest rate and have Vermont lenders sell the mortgages directly to Fannie Mae instead of through the VHFA. The program is expected to begin in September 1993.

\section{Washington}

The Washington State Energy Office, in collaboration with Fannie Mae and 10 Washington public utilities, has designed a pilot program enabling home buyers qualifying under Fannie Mae's Community Home Buyers (CHB) Program to take advantage of utility-builder rebates when purchasing new, electrically heated homes in Washington. Banks approved by Fannie Mae to administer the CHB program will offer loans with flexible underwriting criteria to low- and moderate-income families. The program:

- Allows a higher debt-to-income ratio (up to 33\% of a borrower's gross monthly income)

- Allows borrowers with no credit history to use nontraditional means to establish a good credit record

- Requires a minimum downpayment of $5 \%, 2 \%$ of which can be in the form of a grant or gift

- Waives the requirement that borrowers must have reserve funds to cover mortgage payments for two months.

Under the CHB pilot program, eligible borrowers purchasing new, electrically heated homes may capture utility-incentive payments (normally paid to builders) to defray the cost of the downpayment by up to $2 \%$; at least $3 \%$ of the downpayment must come from a borrower's own funds. Participating builders recruited by the utilities would set aside a percentage of new homes for sale to low- and moderate-income individuals while agreeing to pass the benefit of the utility rebate on to the buyers. Fannie Mae will follow the loans issued through the CHB pilot program for signs of increased risk associated with utilityassisted financing. The pilot program is expected to begin in June 1993. 


\section{EPA's Wisconsin Pilot}

In July 1992, the Environmental Protection Agency's (EPA) Climate Change Grant Program awarded a $\$ 100,000$ grant to the Wisconsin Energy Bureau (WEB) to develop and market a Wisconsin EEM program. The 2-year grant will finance an EEM pilot program in the Madison area along with planning for statewide implementation. An evaluation component to determine information regarding energy savings and environmental benefits also will be completed. When EPA awarded the grant, neither a HERS nor a standardized process for offering EEMs was in place. WEB is working to implement both in cooperation with representatives from state government, utilities, lending institutions, and the real estate and building industries.

\section{Summary}

Through these and other pilot programs, such as those which may be developed to test EEMs options recommended by HUD's Section 946 Task Force, data can be generated that will permit the development of answers to a number of key questions. Such questions include the extent of EEMs market penetration, the effect of efficiency on assessed value, the impact of EEMs on default rates, the nature of the HERS/EEMs linkage, and the most effective communication and marketing strategies for EEMs. 


\section{Actions in Support of HERS and EEMs}

This section is organized into two parts. The first part summarizes activities undertaken by states and regions in HERS and EEMs. The second part summarizes activities by national-level stakeholder organizations.

\section{State Activities}

Table 4 (beginning on page 38) summarizes information on state activities, which is documented in detail in Appendix A. The state activities show development of scaled HERS performance-based systems; utility programs, including demand-side management (DSM), Good Cents, and Super Good Cents programs; efforts to link with EEMs at the state level; and relevant pilot programs at the state level by various federal agencies.

\section{HERS programs}

HERS program activity is widespread in the United States. Seventy-eight percent of the states $(\mathrm{N}=39)$ have some type of HERS program, either statewide or in localities. Figure 1 depicts the geographic distribution of HERS performance-based and certification programs in the United States. As Figure 1 shows, one or more utilities in 22 states (44\% of the states) offer only certification programs; six states (12\%) have only performance-based HERS programs; and 11 states (22\%) have both types of HERS programs. Two-thirds of the states $(\mathrm{N}=33)$ have at least one utility with a certification type of HERS program.

States that have only certification programs are Alabama, Connecticut, Delaware, Florida, Georgia, Idaho, Maine, Massachusetts, Montana, Nevada, North Carolina, New Hampshire, New Jersey, New York, Ohio, Oklahoma, Oregon, Pennsylvania, South Carolina, Washington, Wisconsin, and Wyoming. States that have only performance-based HERS programs are Alaska, Arkansas, Michigan, New Mexico, Vermont and West Virginia. States with both types of HERS programs are Arizona, California, Colorado, Illinois, Indiana, Iowa, Louisiana, Mississippi, Rhode Island, Texas, and Virginia.

The remaining 11 states (22\%) have neither type of HERS program. However, two of them have special energy-efficiency programs (Maryland and Missouri); three are developing HERS programs (Minnesota, North Carolina and South Dakota); two are researching HERS programs (Kansas and Nebraska); and six have expressed an interest in HERS (Hawaii, Kentucky, Maryland, Missouri, North Dakota, and Utah). Only the District of Columbia and Tennessee are not known to have at least expressed an interest in HERS; however, both of these entities have utility DSM programs and Tennessee has been involved in discussions of energy efficiency in refinancing and home improvement loans.

Nineteen states were identified by FmHA as having statewide building codes that meet or exceed CABO Model Energy Code 1989 levels for energy efficiency. These states are California, Connecticut, Florida, Georgia, Idaho, Indiana, Iowa, Kentucky, Maryland, Minnesota, New Mexico, New York, Oregon, Rhode Island, South Carolina, Utah, Virginia, Washington, and Wisconsin. Because HUD/FHA and FmHA mortgages can only be given in states that have building codes at least this stringent according to 1992 legislation, the number of states with additional energy-efficient codes is likely to increase in the future.

All of the states have at least one utility residential DSM program (see Appendix A). From an energyefficiency standpoint, this is heartening. Assuming that utilities want to save energy, DSM programs could, in the future, provide a critical institutional linkage between HERS programs on the one hand and EEMs programs on the other. The fact that DSM programs exist in the states means that they could 


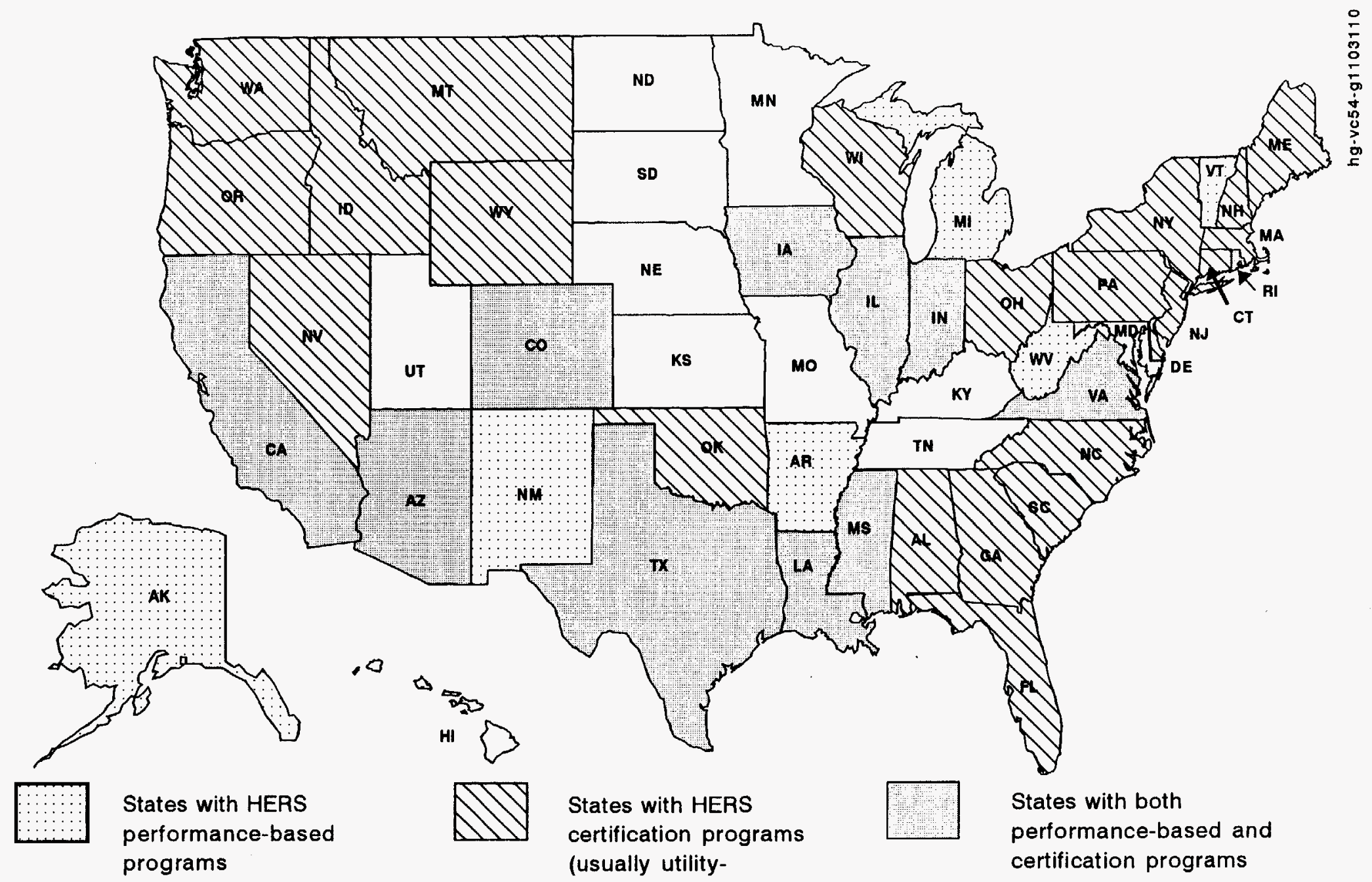

Source: Constructed by the authors based on data reported in Appendix A.

Figure 1. States with HERS programs (either statewide, local-area, or both) (as of 09/93) 
potentially provide a foundation for linking EEMs with HERS programs that is nationwide in scope. Utility DSM programs could provide downpayment assistance, third-party indemnification, rebates for measures, one-stop shopping, and other services to expedite HERS/EEMs program usage. For example, rural electric cooperatives in Kentucky and Tennessee have had some preliminary discussions with Fannie Mae about the role of residential energy efficiency in mortgage refinancing and home improvement loans.

A cautionary note is in order, however. DSM objectives vary widely from one utility to another. Most DSM programs are conducted by electric utilities, yet in many regions homes use natural gas and fuel oil for heating, water heating, and cooking. Although DSM programs may not by themselves be a comprehensive solution, they could be a significant part of it.

The existence of HERS program activity at the state and local levels does not necessarily equate with very many actual ratings being achieved. In fact, most performance-based HERS programs are relatively new. Alaska's started in 1986 and Vermont's in 1987; several others have come into being only in the last two to three years. Good Cents programs have been in existence since 1975, and the Bonneville Power Administration's Super Good Cents program began in 1984. Similarly, DSM programs are a relatively new innovation, having begun about a decade ago. ${ }^{6}$ Nevertheless, the evolution of these programs, and their spread across the country, provide a significant institutional base for energy-efficiency and renewable energy programs for housing.

\section{EEMs programs}

State energy offices (SEOs), utility companies, state housing finance authorities, builders, public utility commissions, and other entities are paying increasing attention to EEMs. ${ }^{7}$ Although EEMs are hypothetically available nationwide, in practice most lenders and consumers are unaware of them or choose not to use them. Figure 2 shows that EEMs are available through lenders in at least 23 states, according to the evidence presented in Appendix A: Alaska, Arizona, Arkansas, California, Colorado, Georgia, Idaho, Illinois, Indiana, Iowa, Maryland, Michigan, Montana, New Mexico, New York, North Carolina, Ohio, Oklahoma, Oregon, Rhode Island, Vermont, Virginia, and Washington. At least one lending institution, and often more than one, is known to provide EEMs in these states.

Special efforts by various organizations to link HERS programs with EEMs exist in 32 states. Variety is the hallmark of these linking efforts. State energy offices are involved in developing linkages with EEMs in 14 states (Arizona, Arkansas, Idaho, Indiana, Iowa, Louisiana, Mississippi, New Jersey, North Carolina, Ohio, Oregon, Vermont, Washington, and Wisconsin). Each of these states is following its own unique path in forging these linkages. For example, in Arkansas the legislature mandated the state energy office to establish an EEMs link with ERH of AR. In Mississippi, the SEO is working with the Cooperative Extension Service and lenders to develop EEMs. In North Carolina, the SEO is negotiating directly with lenders to offer EEMs.

${ }^{6}$ DSM programs began in Wisconsin and California in 1975, according to S. Wiel and in 1978, according to R. Cavanaugh (Maize and McCaughey 1993, p. 4). The National Association of Regulated Utility Commissioners Conservation Committee was established in 1984 (Maize and McCaughey 1993).

\footnotetext{
${ }^{7}$ Existing EEMs programs are summarized in Appendix E.
} 


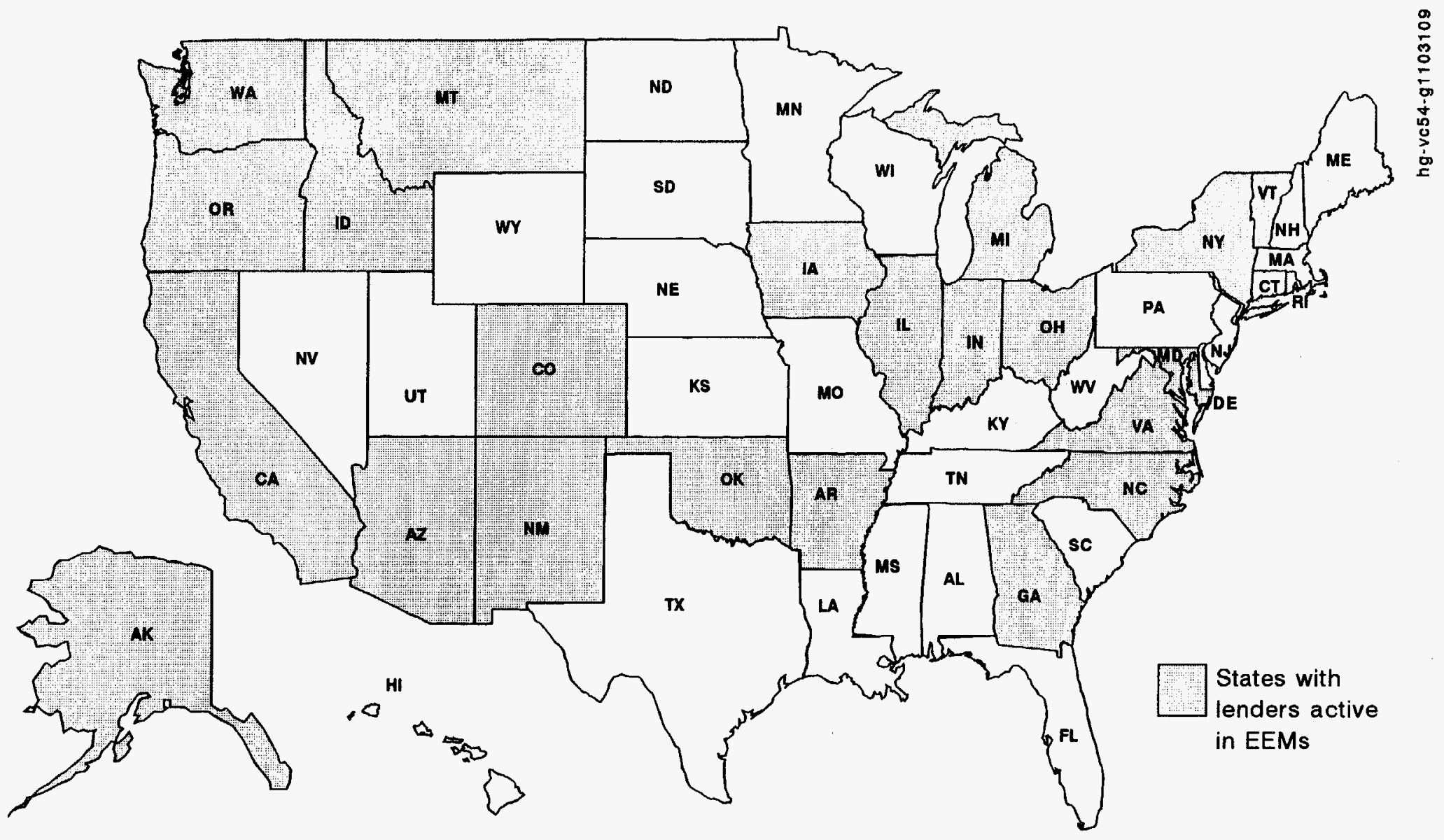

Source: Constructed by the authors based on data reported in Appendix A.

Figure 2. States with one or more lending institutions that facilitate access to EEMs* (as of 09/93)

"Everywhere in the nation, mortgages that take energy efficiency into account are provided, guaranteed, or insured by HUD/FHA, DVA, FmHA, and FHLMC. 
In Alaska, Rhode Island, and Vermont, state housing finance authorities are involved in forming linkages between HERS and EEMs. In Washington State, the Northwest Power Planning Council (a regional entity) and BPA are also involved in EEMs linkages. In states such as Colorado, Georgia, Maryland, and Minnesota, utilities have worked directly with lenders. In Kansas, Montana, and Illinois, state housing agencies are developing the linkage.

It is too early in the evolution of EEM institutional arrangements to determine whether a particular model will work best in most parts of the country. The most mature programs (especially Alaska, Vermont, and Rhode Island) involve state housing finance authorities. On the other hand, California's new CHEERS program has developed a mortgage committee to work out the linkage with EEMs, while Colorado may establish a Colorado Energy Mortgage Authority (CEMA) as a separate entity which itself provides the linkage. These varied modes of establishing working relationships among the various stakeholder groups at the state and local levels will require some experimentation before acceptable structures can be achieved.

\section{Other Actions}

Numerous actions to promote HERS/EEMs have taken place since March 1992, when the National Collaborative issued its report. Table 5 (beginning on page 51) summarizes actions taken from spring 1992 to spring 1993 by the major stakeholder groups. Details are documented in Appendix B. These activities are organized into six categories: (1) building awareness, (2) HERS/EEMs network participation, (3) proactive initiatives, (4) reviews and changes in policies/practices, (5) pilot testing, and (6) studies.

\section{Building awareness}

Most organizations have been involved in raising the levels of awareness on HERS and EEMs among their own members. A few have gone beyond this to actively educate other stakeholders, including:

- Energy Rated Homes of America, which hosted a national conference titled "Jump Starting HERS and EEMs" in the spring of 1993

- The Consumer Federation of America, which held a public forum in 1992 to provide a venue for commentary on A Blueprint for Action

- The National Association of Home Builders and Southern Electric International, which held sessions on HERS/EEMs at their annual conventions in 1992 and 1993

- HUD/FHA, which trained lenders in EEMs concepts in 1993

- The Alliance to Save Energy and Freddie Mac, which responded to hundreds of inquiries from the public in response to media stories on EEMs

- Energy Rated Homes of Virginia, which initiated the HERS Provider Information Network.

Increasing the knowledge among the implementing organizations is a necessary first step in developing a national program of HERS and EEMs. Far preferable to a government program in which information flows out from a state or federal agency to stakeholders is a system of consistent, credible information from trusted sources-in this case, the very trade and professional associations whose role it is to monitor members' interests. With HERS and EEMs, the stakeholders themselves are providing the information. 


\section{Network participation}

Most members of the National Collaborative and its Technical Advisory Committees have remained active in an ongoing network on HERS and EEMs. Out of these interactions, the HERS Council has been instituted and has broad representation. Although the Collaborative did not include product manufacturer representatives, the HERS Council does. Every indication is that this network has been permanently established.

\section{Proactive initiatives}

Among the most significant initiatives are the following:

- Establishment of the HERS Council (most Collaborative organizations are involved)

- Edison Electric Institute's (EEI) Three-E Program

- Fannie Mae's EEMs pilot tests

- Energy Rated Homes of America (ERHA), Fannie Mae, and Freddie Mac analyses of existing HERS and EEMs data

- Training initiatives for lenders (FHA/HUD) and raters (ERHA)

- Regional Good Cents Roundtables (SEI)

- Influencing the Energy Policy Act and National HERS/EEMs policy (NA-EEM-SCo and NRDC).

A notable feature of these activities is that they were initiated by the organizations themselves and relied very little on federal funding. This suggests that the activities are not only acceptable to the stakeholder groups, but are also more likely to be self-sustaining than if they had been government-initiated efforts.

\section{Reviews and changes in policies and practices}

About half of the stakeholder organizations were involved in these actions. Several carried out policy changes, such as passing formal resolutions, or attempted to influence national or organizational policies to make them more favorable to HERS and EEMs. Others changed their own existing practices to make them more effective (e.g., ERHA's rater training) or more in line with anticipated future HERS guidelines (e.g., Good Cents software). In all cases, changes were voluntary and conducive to the increased acceptance and adoption of HERS and EEMs, although cautionary notes were sounded by AARP and real estate professionals on such issues as property value protection and consumer disclosure.

\section{Pilot testing of EEMs}

Most pilot tests involve EEMs. Vermont's Housing Finance Agency and Fannie Mae were early in fielding an EEMs pilot program. The array of other pilots-developed in partnership between Fannie Mae and various state, local, and utility entities-is testimony to the creative, innovative process currently characterizing EEMs.

The involvement of state housing finance authorities and utility DSM programs in EEMs pilot tests may be a harbinger of future EEMs institutional arrangements. Other key players may emerge as appraisers, real estate agents, home inspectors, federal agency regional offices, and state and local jurisdictions adjust and target their programs.

Pilot tests in five states are mandated by EPAct, as already discussed. HUD/FHA may foster EEMs pilots in additional states to test several other EEMs concepts that the HUD Section 946 Task Force has examined and that HUD/FHA may recommend. 
This pilot testing is invaluable in providing field validation for specific methods of determining energy efficiency, cost-effectiveness, and efficacy of features. If properly designed, pilot tests will provide data for evaluation of the impact of EEMs on default rates, and thus on portfolio risk. Additional information should answer questions about the number of home buyers interested in EEMs, the most effective institutional arrangements, and other key questions.

\section{Evaluation of HERS and EEMs Activities}

The Energy Policy Act of 1992 requires that activities carried out under the statute be documented. Table 2 presents a preliminary assessment of evaluation activities needed to fulfill statutory requirements for HUD/FHA EEMs pilots. Table 3 does the same for the residential energy efficiency ratings voluntary guidelines which DOE must issue and for other related activities required of DOE.

As resources are available, monitoring and evaluation activities on both the technical and programmatic aspects of HERS and EEMs activities and pilots will be conducted.

It is likely that state, federal, and other organizations will be involved in evaluation planning, data collection, analysis, and reporting.

\section{Related Studies}

Few studies have been completed. Vories and George (1991) surveyed HERS activity in the states. ${ }^{8}$ Argonne National Laboratory is working on a HERS/EEMs-related project in collaboration with the Energy Resource Center at the University of Illinois, Chicago, to conduct a cost-benefit analysis of two south Chicago apartment buildings, one of which has been retrofitted with energy-efficiency features. In addition, the study will examine barriers to incorporating energy-efficiency improvements in low-income housing and will assess whether HERS, when linked with EEMs, increases access to affordable housing. This study is being funded by DOE's Office of Building Technologies through Oak Ridge National Laboratory.

A 1992 study on stakeholder attitudes toward HERS programs was conducted by the Burruss Institute of Public Service at Kennesaw State College in Marietta, Georgia. The study, entitled Home Energy Efficiency and the Georgia Energy Code, surveyed Georgia real estate agents, utility representatives, bankers, and code inspectors. The results showed that a rating system would be favored by most utility. representatives, bankers, and code inspectors $(94 \%, 86 \%$, and $85 \%$, respectively). A system of this type would be opposed by most real estate agents ( $80 \%$ opposed).

Barakat and Chamberlin have been conducting a project with Infinite Energy, Energy Rated Homes of America, and Scanada Consultants, Ltd., funded by the Ontario Ministry of Environment and Energy, Ontario Hydro, and Consumers Gas. Its purpose is to develop a strategy for developing home energy ratings in the province of Ontario. The project's four tasks are:

1. A review of HERS experience in North America and overseas

2. An assessment of lessons learned from this experience

3. An analysis of market conditions and stakeholder views regarding HERS in Ontario

4. Development of strategic options for developing a HERS in Ontario.

${ }^{8}$ Appendix A's introduction describes studies used in this analysis. 
Products include an annotated bibliography of HERS literature and detailed descriptions of 14 HERS programs. The project's final report will be available late in 1993.

Many more studies can be expected as the monitoring and evaluation of HERS and EEMs field activities increase.

Table 2. Evaluation Requirements - HUD/FHA EEMs Pilots

\begin{tabular}{|c|c|}
\hline Statutory Requirement & Evaluation Activity \\
\hline $\begin{array}{l}\text { The Secretary of HUD, not later than } 6 \text { months } \\
\text { after the date of enactment of EPAct (by } \\
\text { April 24, 1993) shall establish an energy-efficient } \\
\text { mortgage pilot program in five states to promote } \\
\text { the purchase or existing energy-efficient } \\
\text { residential buildings and the installation of cost- } \\
\text { effective improvements in existing residential } \\
\text { buildings. }\end{array}$ & $\begin{array}{l}\text { Document the implementation of the } \\
\text { program, including state activities. }\end{array}$ \\
\hline $\begin{array}{l}\text { 2. Not later than } 9 \text { months after the enactment of } \\
\text { EPAct (by July } 24,1993 \text { ), the Secretary, in } \\
\text { consultation with the Secretary of DOE, shall } \\
\text { establish and implement a program for training } \\
\text { personnel at relevant lending agencies, real estate } \\
\text { companies, and other appropriate organizations } \\
\text { regarding the benefits of energy efficient } \\
\text { mortgages and the operation of the pilot program. }\end{array}$ & $\begin{array}{l}\text { Describe the implementation of the } \\
\text { training program for lenders, real estate } \\
\text { professionals, and others } \\
\text { Document the dates, places, and } \\
\text { number of trainees at each training } \\
\text { session } \\
\text { - Summarize the number of trained } \\
\text { professionals by type in each state, and } \\
\text { in total, over time } \\
\text { - Other variables, as defined. }\end{array}$ \\
\hline $\begin{array}{l}\text { 3. Not later than } 18 \text { months after the date of } \\
\text { enactment of EPAct (by April 24, 1994), the } \\
\text { Secretary shall prepare and submit a report to } \\
\text { Congress describing the effectiveness and } \\
\text { implementation of the EEM pilot program as } \\
\text { described, and assessing the potential for } \\
\text { expanding the pilot program nationwide. }\end{array}$ & $\begin{array}{l}\text { Describe program effectiveness: } \\
\text { - Housing affordability } \\
\text { - Energy and cost savings } \\
\text { - Business development impacts (cost of } \\
\text { measures installed, who installed the } \\
\text { measures) } \\
\text { - Number of EEMs, by state and total } \\
\text { - Analysis of default rates on EEMs } \\
\text { compared with regular mortgages } \\
\text { - Other variables, as defined. }\end{array}$ \\
\hline $\begin{array}{l}\text { 4. Not later than the expiration of the 2-year period } \\
\text { beginning on the date of the implementation of } \\
\text { the energy-efficient mortgage pilot program (by } \\
\text { April 24, 1995), the Secretary of HUD shall } \\
\text { expand the pilot program on a nationwide basis } \\
\text { to include new residential housing, unless this } \\
\text { expansion is determined not to be practicable. }\end{array}$ & $\begin{array}{l}\text { Define program practicality: } \\
\text { - Define how program would be } \\
\text { expanded to new housing } \\
\text { - Define problem areas (actual and } \\
\text { potential) } \\
\text { - Determine practicality of program for } \\
\text { new housing } \\
\text { - Determine practicality of expansion } \\
\text { (operational definition of practicality is } \\
\text { required). }\end{array}$ \\
\hline
\end{tabular}


Table 3. Evaluation Requirements - DOE Residential Energy Efficiency Ratings Voluntary Guidelines

\begin{tabular}{||l|l||}
\hline \multicolumn{1}{|c|}{ Statutory Requirement } & \multicolumn{1}{|c|}{ Evaluation Activity } \\
\hline $\begin{array}{l}\text { Establish (by April 24, 1994) protocols and } \\
\text { procedures for: } \\
\text { Certification of the technical accuracy of } \\
\text { building energy analysis tools used to } \\
\text { determine energy efficiency ratings } \\
\text { Training of personnel conducting energy } \\
\text { efficiency ratings }\end{array}$ & $\begin{array}{l}\text { Process evaluation and documentation of the } \\
\text { establishment of protocols and procedures } \\
\text { required (include number of trained raters, } \\
\text { documentation of training sessions, etc.); } \\
\text { include both state and federal actions. }\end{array}$ \\
$\begin{array}{l}\text { Data collection and reporting } \\
\text { Quality control } \\
\text { Monitoring and evaluation. }\end{array}$ & \\
\hline $\begin{array}{l}\text { Establish a program of technical assistance to } \\
\text { state and local organizations to encourage } \\
\text { adoption and use of REERS consistent with the } \\
\text { voluntary guidelines (by October 24, 1994). }\end{array}$ & Based on the process evaluations above. \\
\hline $\begin{array}{l}\text { Report to Congress (by October 24, 1995) } \\
\text { describing actions taken by DOE and other } \\
\text { federal agencies to implement the program. }\end{array}$ & Based on the process evaluations above. \\
\hline $\begin{array}{l}\text { Recommend to Congress the feasibility of } \\
\text { requiring a minimum energy efficiency rating as } \\
\text { a prerequisite to receiving federally assisted, } \\
\text { guaranteed, or insured mortgages. }\end{array}$ & Define data needed to assess feasibility. \\
\hline
\end{tabular}




\section{Conclusions}

At the National Collaborative's initial meetings, members of the mortgage community said that a fully implemented EEMs program awaited a credible nationwide HERS program. Energy-efficiency advocates, on the other hand, said the lack of a uniform EEMs program was impeding the further development of HERS. Activities since the Collaborative issued its Blueprint suggest that development is needed simultaneously in both HERS and EEMs so a positive outcome can be achieved. Ratings provide mortgage bankers with a dependable basis for monetary savings on utility costs for use in writing mortgage terms.

Recommendations of the National Collaborative are being implemented through legislative mandates and voluntary actions:

- EPAct and other legislation relied on the Blueprint's ideas and language in setting forth technically correct requirements for residential energy-efficiency ratings and EEMs programs.

- The HUD Section 946 Task Force used the Blueprint's recommendations and technical issue papers prepared by Collaborative members as a starting point for its work.

- The HERS Council is building on the Collaborative's work by following through on the HERS technical issues, education and training needs, the HERS/EEMs linkage, and institutional issues.

- Protocols for certifying HERS calculation tools and voluntary guidelines are being developed in a partnership involving DOE, NREL, and the HERS Council. The Council also will develop a voluntary system for certifying HERS software tools and accrediting HERS programs.

- HUD/FHA will likely continue an EEMs Working Group after the Section 946 Task Force recommendations have been published. The EEMs Working Group would provide a venue for federal agencies and federally-chartered financial institutions that issue, guarantee, and insure mortgages to continue discussion of EEMs practices and monitor the results of the EEMs pilot tests. The EEMs Working Group could also work with the HERS Council, particularly its HERS/EEMs Linkage Committee, to foster the linkage of HERS with EEMs.

- DOE is also working to establish a multiagency HERS/EEMs management team with representation from HUD, DOE's Office of Energy Efficiency and Renewable Energy, NREL, EPA, D\&R International (administrator for the HERS Council), and the DOE support offices to provide a forum for coordinating ongoing efforts in which the federal government is involved.

Although some expected that the National Collaborative could and should have, in its 10 meetings, resolved the items that still remain to be accomplished, most recognize that the invention of such a complex program will take time. That progress is being made on so many fronts simultaneously is testimony to the widespread interest in the potential of HERS and EEMs in saving energy, protecting the environment, improving housing quality and affordability, and developing the economy.

As the National Collaborative determined, the lack of adequate data and analyses has impeded the development and implementation of this promising program. Analysis and evaluation remain to be accomplished on such critical questions as the national impacts of EEMs on utility costs, energy savings, environmental protection, housing affordability, and mortgage default rates. Consumer perceptions, preferences, and behavior with respect to energy investments and real estate finance have yet to be researched and understood. The behavior of those occupying homes significantly affects energy 
consumption, which, in identical housing units, can vary by a factor of two or more. The ways in which occupant energy behavior will affect HERS and EEMs processes remains to be determined.

Other questions include the following:

- Should utility DSM programs be developed in partnership with EEMs?

- Can ways be found to interest real estate professionals, appraisers, home inspectors, and lenders themselves in the EEMs process?

- How should HERS programs be linked with DOE's Weatherization Assistance Programs (WAP), HUD rehabilitation programs, and programs that address FHA housing default prevention and solar strategies?

- How can the number of qualified raters be increased?

- How can the national organizations of stakeholders provide support to the FHA EEMs pilot programs through their members' organizations in pilot states?

- What action and information is needed to develop data for use in routine valuation procedures?

A "critical mass" appears to have formed around the HERS/EEMs issue. The institutional mechanisms needed to address and answer questions that remain are now in place. Virtually all members of the National Collaborative have continued their HERS/EEMs activities since the Blueprint came out. In many parts of the nation, builders, lenders, the real estate community, utility companies, HERS providers, and state and local energy officials are coming together in collaborative processes to learn about the complexities and, in step with national efforts, devise ways to resolve them. State legislators are getting involved in several jurisdictions.

This approach to HERS/EEMs is the single most persuasive factor in arriving at the conclusion that an effective and lasting system of finance for residential energy improvements and efficient new housing ultimately will be established. Whatever the final shape of this system, it will be feasible and workable to the extent that the organizations which operate it will have designed it. It will be acceptable for the same reason. If the effects that seem likely are realized, such a system will prove beneficial to all. 
Table 4. Summary of EEMs/HERS Activities by State

\begin{tabular}{|c|c|c|c|c|c|c|c|c|c|}
\hline States & $\begin{array}{c}\text { Scaled HERS } \\
\text { Programs }\end{array}$ & $\begin{array}{c}\text { Utility } \\
\text { Programs }\end{array}$ & $\begin{array}{c}\text { Linkage with } \\
\text { EEMS }\end{array}$ & $\begin{array}{c}\text { Fannie } \\
\text { Mae }\end{array}$ & $\begin{array}{c}\text { Freddie } \\
\text { Mac }\end{array}$ & FHA/HUD & $\begin{array}{l}\text { Pilot } \\
\text { Tests } \\
\end{array}$ & EPA & FmHA \\
\hline Alabama & & \begin{tabular}{|l} 
- Good Cents: \\
17 utilities \\
- DSM' programs: \\
3 utilities \\
- Wise Home \\
Program: \\
1 utility \\
\end{tabular} & & & & & & & \\
\hline Alaskn & $\begin{array}{l}\mathrm{ERH}^{2} \text { of } \mathrm{AK} \\
(1986)\end{array}$ & $\begin{array}{l}\text { DSM prograns: } \\
2 \text { utilities }\end{array}$ & $\begin{array}{l}\text { FHA EEMs } \\
\text { through AK } \\
\text { Housing Finanoe } \\
\text { Corp. } \\
\text { - } 18 \text { lending institu- } \\
\text { toons active in } \\
\text { EEMs }\end{array}$ & $\begin{array}{l}\text { Accepts ERH of } \\
\text { AK ratings for } \\
\text { EEMs }\end{array}$ & $\begin{array}{l}\text { Accepts ERH of AK } \\
\text { ratings for EEMs }\end{array}$ & $\begin{array}{l}\text { Recognizes for EEMS } \\
\text { - The AK energy } \\
\text { conservation } \\
\text { standard for new } \\
\text { housing } \\
\text { - ERH of AK 4- to } \\
\text { double 5-star } \\
\text { ratings } \\
\end{array}$ & $\begin{array}{l}\text { HUD/FHA EEMs } \\
\text { pilot state }\end{array}$ & & \\
\hline Arizona & $\begin{array}{l}\text { AzHERS }^{4}(1990) \\
\text { (CALRES/Az) }\end{array}$ & $\begin{array}{l}\text { - Good Cents: } \\
2 \text { utilities } \\
\text { - DSM programs: } \\
4 \text { utilities } \\
\text { - Salt River Project } \\
\text { (Climate Craftied } \\
\text { Homes) (1990) } \\
\text { (cerufication } \\
\text { program) } \\
\text { - Energy Advantage } \\
\text { Home Program: } \\
1 \text { uitity } \\
\end{array}$ & $\begin{array}{l}\text { - AzHERS working } \\
\text { on EEMs linkage } \\
\text { for new and } \\
\text { existing homes } \\
\text { through local banks } \\
\text { and mortgage } \\
\text { companies } \\
\text { - EEMs bave been } \\
\text { processed in the } \\
\text { state }\end{array}$ & $\begin{array}{l}\text { Accepts AxHiERS } \\
\text { for EEMs }\end{array}$ & & $\begin{array}{l}\text { Accepts AzHERS for } \\
\text { EEMs }\end{array}$ & & & \\
\hline
\end{tabular}

${ }^{3}$ The number of active lending inscitutions shown are only those known to the authors and may not be exhaustive

AzHERS - Arizona Home Energy Rating System 
Table 4. Summary of EEMs/HERS Activities by State (cont'd.)

\begin{tabular}{|c|c|c|c|c|c|c|c|c|c|}
\hline States & $\begin{array}{l}\text { Scaled HERS } \\
\text { Programs }\end{array}$ & $\begin{array}{l}\text { Utility } \\
\text { Programs }\end{array}$ & $\begin{array}{l}\text { Linkage with } \\
\text { EEMS }\end{array}$ & $\begin{array}{c}\text { Fannie } \\
\text { Mae }\end{array}$ & $\begin{array}{l}\text { Freddie } \\
\text { Mac }\end{array}$ & FHA/HUD & $\begin{array}{l}\text { Pilot } \\
\text { Tests }\end{array}$ & EPA & FmHA \\
\hline Arkansas & ERH of AR (1987) & $\begin{array}{l}\text { DSM programs: } \\
4 \text { utilities }\end{array}$ & $\begin{array}{l}1993 \text { legislation } \\
\text { tequires SEO to } \\
\text { develop and imple- } \\
\text { ment an EEMs } \\
\text { pilot program } \\
4 \text { lending institu- } \\
\text { toos active in } \\
\text { EEMs } \\
\end{array}$ & $\begin{array}{l}\text { Accepts ERH of } \\
\text { AR ratings for } \\
\text { EEMs }\end{array}$ & $\begin{array}{l}\text { Acceps ERH of AR } \\
\text { raungs for EEMs }\end{array}$ & $\begin{array}{l}\text { Accepts ERH of AR } \\
\text { ratings for EEMs }\end{array}$ & $\begin{array}{l}\text { HUD/FHA EEMs } \\
\text { pilot state }\end{array}$ & & \\
\hline California & $\begin{array}{l}\text { CHEERS }^{6} \\
\text { (1991) }\end{array}$ & 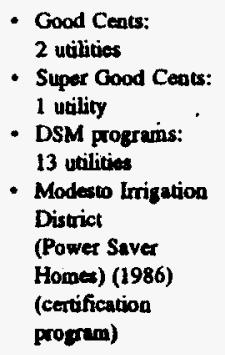 & $\begin{array}{l}\text { CHEERS Mortgage } \\
\text { Committee is } \\
\text { deveioping EEMs } \\
\text { guidolines } \\
\text { - lending insuitu- } \\
\text { tions active in } \\
\text { EEMs }\end{array}$ & $\begin{array}{l}\text { Accepss CHEERS } \\
\text { for EEMs }\end{array}$ & $\begin{array}{l}\text { Accepts CHEERS for } \\
\text { EEMs }\end{array}$ & $\begin{array}{l}\text { Accepts CHEERS for } \\
\text { EEMs }\end{array}$ & $\begin{array}{l}\text { HUD/FHA EEMs } \\
\text { pilot state }\end{array}$ & & $\begin{array}{l}\text { State building cude } \\
\text { meets/excoeds } \\
\text { CABO MEC } 89\end{array}$ \\
\hline
\end{tabular}

SSEO - State energy office (regardless of the specific designation of the office in each state)

CABO MEC - Council of American Building Officials Model Energy Code 
Table 4. Summary of EEMs/HERS Activities by State (cont'd.)

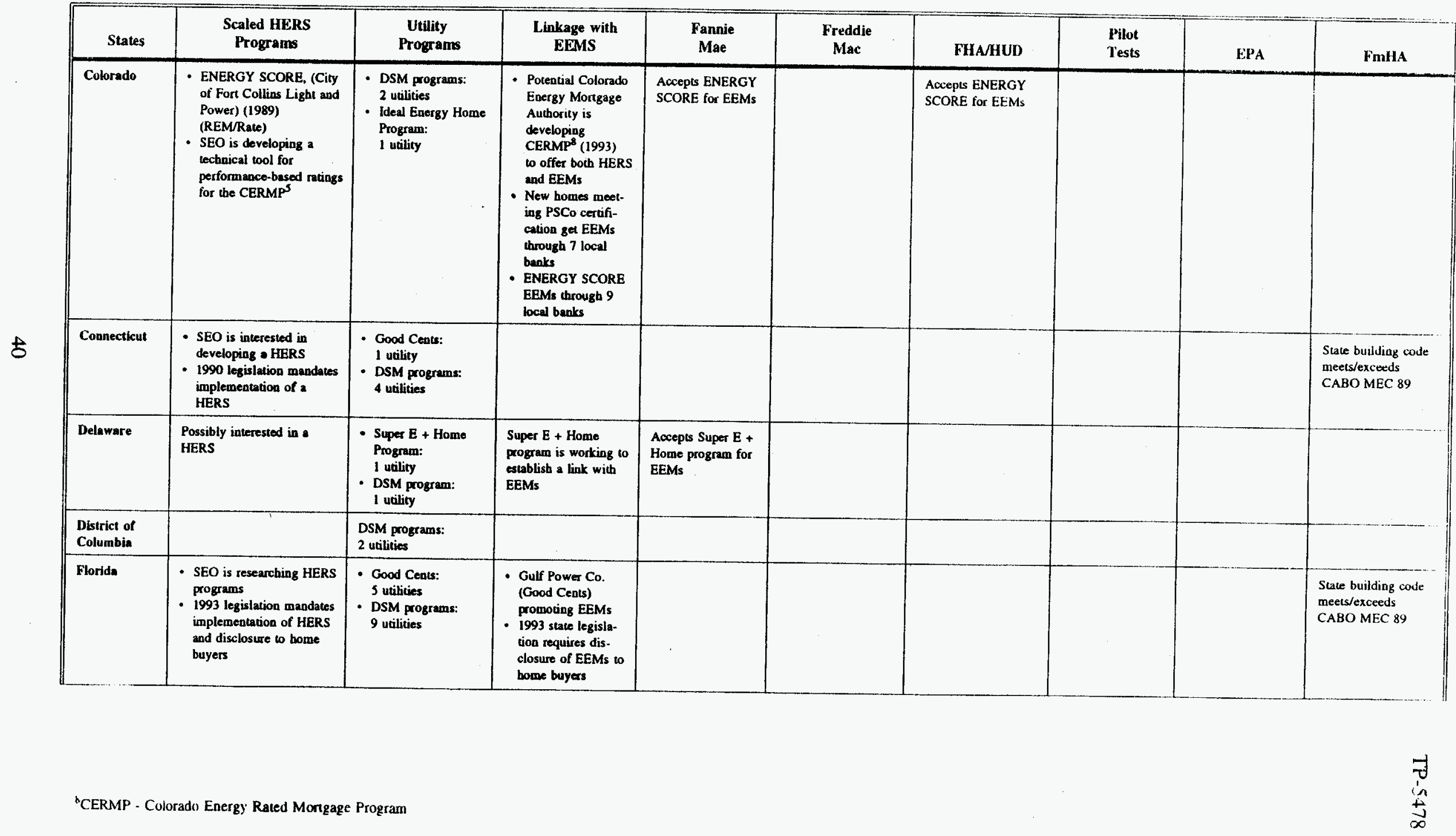


Table 4. Summary of EEMs/HERS Activities by State (cont'd.)

\begin{tabular}{|c|c|c|c|c|c|c|c|c|c|}
\hline States & $\begin{array}{c}\text { Scaled HERS } \\
\text { Programs }\end{array}$ & $\begin{array}{l}\text { Utility } \\
\text { Programs }\end{array}$ & $\begin{array}{l}\text { Linkage with } \\
\text { EEMS }\end{array}$ & $\begin{array}{l}\text { Fannie } \\
\text { Mae }\end{array}$ & $\begin{array}{c}\text { Freddie } \\
\text { Mac }\end{array}$ & FHA/HUD & $\begin{array}{l}\text { Pilot } \\
\text { Tests }\end{array}$ & El'A & Finfla \\
\hline Georgia & & $\begin{array}{l}\text { - Good Ceats: } \\
41 \text { utilities } \\
\text { - DSM programs: } \\
14 \text { uujlities } \\
\text { - EnergyWise Home } \\
\text { program: } \\
1 \text { urikity }\end{array}$ & $\begin{array}{l}\text { GA Power Co. linked } \\
\text { with EEMs through } \\
\text { GMAC }^{9}\end{array}$ & & & & & & $\begin{array}{l}\text { Stute building code } \\
\text { rueets/exceeds } \\
\text { CABO MEC } 89\end{array}$ \\
\hline Hawaii & $\begin{array}{l}\text { - SEO is interested in } \\
\text { developing a HERS } \\
\text { program } \\
\text { State increased } \\
\text { SDHW } 10 \text { tax credits to } \\
35 \% \text { (1990) } \\
\text { - State requires SDHW in } \\
\text { bomes subsidized by the } \\
\text { state or locatod on state } \\
\text { land (1993) }\end{array}$ & $\begin{array}{l}\text { DSM programs: } \\
\text { S utilicies }\end{array}$ & $\begin{array}{l}\text { SEO is pursuing } \\
\text { EEMs linkage through } \\
\text { major leaders in } \mathrm{HI}\end{array}$ & & 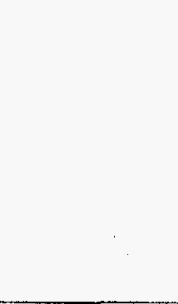 & $\begin{array}{l}\text { Allows } 1 \% \text { stretch for } \\
\text { FHA-approved SDHW } \\
\text { systems in new homes } \\
(1991)\end{array}$ & & & \\
\hline Idaho & $\begin{array}{l}\text { - SEO is interested in a } \\
\text { HERS } \\
\text { - MHAP } \\
\text { - } \text { REEMS }^{11}(1991) \\
(1991)\end{array}$ & $\begin{array}{l}\text { - Good Ceats: } \\
19 \text { unilicies } \\
\text { - Super Good Cents: } \\
\text { 16 utilities } \\
\text { - DSM programs: } \\
2 \text { uvilities } \\
\text { - MHAP (1991) } \\
\text { - REEMS (1991) }\end{array}$ & $\begin{array}{l}\text { - MHAP homes } \\
\text { qualify for EEMs } \\
\text { through FHA } \\
\text { - REEMS pending } \\
\text { - SEO working to } \\
\text { integrate EEMs into } \\
\text { BPA efforts } \\
\text { - } 6 \text { baniss active in } \\
\text { EEMs }\end{array}$ & REEMS pending & REEMS pending & $\begin{array}{l}\text { - Insures MHAP } \\
\text { mortgages } \\
\text { - REEMS pendiog }\end{array}$ & & & $\begin{array}{l}\text { Stite building code } \\
\text { meets/exceeds } \\
\text { CABO MEC } 89\end{array}$ \\
\hline Dlinois & $\begin{array}{l}\text { Energy Wise Homes of } I L \text {, } \\
\text { pilots in Lake County and in } \\
\text { East-central part of stale } \\
\text { (199I) (REM/Rate) }\end{array}$ & $\begin{array}{l}\text { - Good Cents: } \\
1 \text { uality } \\
\text { DSM programs: } \\
8 \text { utilities }\end{array}$ & $\begin{array}{l}\text { SEO pursuing EEMs } \\
\text { through } 5 \text { local banks } \\
\text { and the Illinois } \\
\text { Housing and } \\
\text { Developmeat } \\
\text { Authority }\end{array}$ & & & & $\begin{array}{l}\text { HERS pilot in } \\
\text { Lake County and } \\
\text { East-central part of } \\
\text { stace }\end{array}$ & & \\
\hline
\end{tabular}


Table 4. Summary of EEMs/HERS Activities by State (cont'd.)

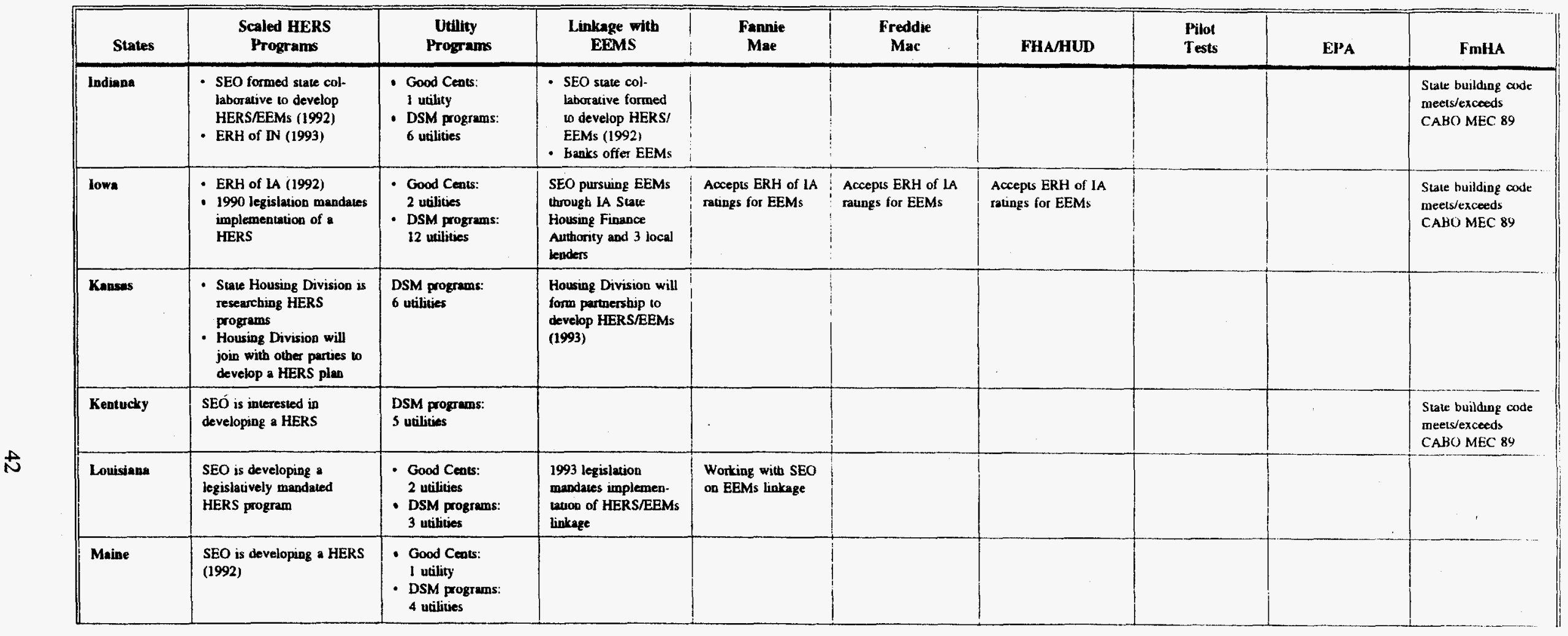


Table 4. Summary of EEMs/HERS Activities by State (cont'd.)

\begin{tabular}{|c|c|c|c|c|c|c|c|c|c|}
\hline States & $\begin{array}{l}\text { Scaled HERS } \\
\text { Programs }\end{array}$ & $\begin{array}{l}\text { Utility } \\
\text { Proprams }\end{array}$ & $\begin{array}{l}\text { Linkage with } \\
\text { EEMS }\end{array}$ & $\begin{array}{l}\text { Fannie } \\
\text { Mae }\end{array}$ & $\begin{array}{l}\text { Freddie } \\
\text { Mac }\end{array}$ & FHAMUD & $\begin{array}{l}\text { Pilot } \\
\text { Tests }\end{array}$ & EPA & FmHA \\
\hline Maryland & $\begin{array}{l}\text { SEO is interested in } \\
\text { developing a HERS after } \\
\text { uxility programs are in place }\end{array}$ & 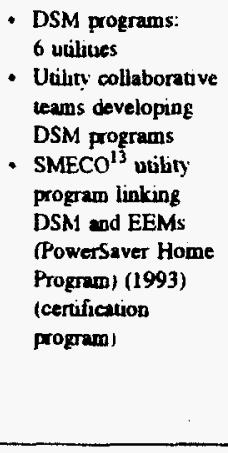 & 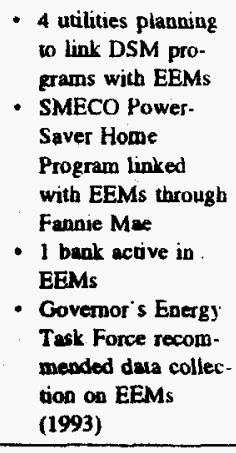 & $\begin{array}{l}\text { - Acceptance of } \\
\text { utidily DSM } \\
\text { programs for } \\
\text { EEMs pending } \\
\text { - SMECO program } \\
\text { approved } \\
\text { - SMECO program } \\
\text { is an EEMs pilot } \\
\text { tesi }\end{array}$ & $\begin{array}{l}\text { - Acceptance of } \\
\text { uuility DSM } \\
\text { programs for } \\
\text { EEMs pending } \\
\text { - SMECO programs } \\
\text { Sapproved } \\
\text { - SMECO program } \\
\text { is an EEMs pilot } \\
\text { test }\end{array}$ & $\begin{array}{l}\text { - Acceplance of } \\
\text { utiliry DSM } \\
\text { programs for EEMs } \\
\text { pendigg } \\
\text { - SMECO program } \\
\text { approved }\end{array}$ & $\begin{array}{l}\text { Fannie Mae EEMs } \\
\text { pilot test } \\
\text { (SMECO) }\end{array}$ & & $\begin{array}{l}\text { State building code } \\
\text { meetserceceds } \\
\text { CABO MEC } 89\end{array}$ \\
\hline Massachusetts & $\begin{array}{l}\text { SEO is researching HERS } \\
\text { programs }\end{array}$ & 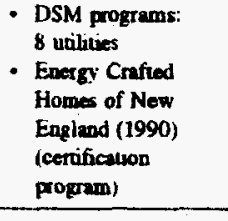 & & & & & & & \\
\hline Michigan & 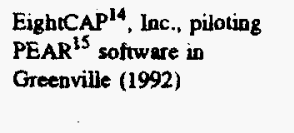 & $\begin{array}{l}\text { DSM proprams: } \\
5 \text { utilicies }\end{array}$ & $\begin{array}{l}\text { Community Action } \\
\text { Program essablisbed } \\
\text { EEMs linkage with } \\
\text { one local benk }\end{array}$ & & & & $\begin{array}{l}\text { HERS pilot in } \\
\text { Greenville, MI }\end{array}$ & & \\
\hline
\end{tabular}

${ }^{13}$ SMECO - Southern Maryland Elecric Cooperative

${ }^{14}$ CAP - Community Action Program

${ }^{15}$ PEAR - Program for Energy Analysis of Residences (software package) 
Table 4. Summary of EEMs/HERS Activities by State (cont'd.)

\begin{tabular}{|c|c|c|c|c|c|c|c|c|c|}
\hline States & $\begin{array}{l}\text { Scaled HERS } \\
\text { Programs }\end{array}$ & $\begin{array}{l}\text { Utility } \\
\text { Programs }\end{array}$ & $\begin{array}{l}\text { Linkage with } \\
\text { EEMS }\end{array}$ & $\begin{array}{l}\text { Fannie } \\
\text { Mae }\end{array}$ & $\begin{array}{l}\text { Freddie } \\
\text { Mac }\end{array}$ & FHAMIUD & $\begin{array}{l}\text { Pilot } \\
\text { Tests }\end{array}$ & EPA & FmHA \\
\hline Minnesola & . & $\begin{array}{l}\text { DSM programs: } \\
21 \text { utilities } \\
\text { - NSP }{ }^{16} \text { Gas and } \\
\text { Electric and } \\
\text { Minnegasco are } \\
\text { developing a } \\
\text { certification } \\
\text { program for new } \\
\text { homes (1993) }\end{array}$ & $\begin{array}{l}\text { When developed, } \\
\text { urilities plan to link } \\
\text { cerification prograns } \\
\text { with EEMs }\end{array}$ & & & & & & $\begin{array}{l}\text { Scate building code } \\
\text { meets/exceds } \\
\text { CABO MEC } 89\end{array}$ \\
\hline Mississippi & ERH of MS & $\begin{array}{l}\text { - Good Cents: } \\
12 \text { utilities } \\
\text { - DSM programs: } \\
2 \text { utilities } \\
\text { - Gas Mark: } \\
1 \text { utility }\end{array}$ & $\begin{array}{l}\text { SEO and Cooperative } \\
\text { Exteasion Service } \\
\text { developing statewide } \\
\text { linkages with local } \\
\text { leoders to offer EEMs } \\
\text { through DVA, } \\
\text { HUDAFHA, and } \\
\text { FmHA }\end{array}$ & $\begin{array}{l}\text { Accepts ERH of } \\
\text { MS ratings for } \\
\text { EEMs }\end{array}$ & $\begin{array}{l}\text { Accepts ERH of MS } \\
\text { ratings for EEMs }\end{array}$ & $\begin{array}{l}\text { Accepts ERH of } \\
\text { MS ratings for } \\
\text { EEMs } \\
\text { Working with SEO } \\
\text { on EEMs linkage }\end{array}$ & & & $\begin{array}{l}\text { Working with SEO } \\
\text { on EEMs linkage }\end{array}$ \\
\hline Missouri & SEO is interestiod in HERS & $\begin{array}{l}\text { DSM programs: } \\
6 \text { urilities } \\
\text { - Kansas City Power } \\
\text { and Light and } \\
\text { builder certification } \\
\text { program (Save } \\
\text { America's Valuable } \\
\text { Energy) (1982) }\end{array}$ & & & & & & & \\
\hline Montana & $\begin{array}{l}\text { - SEO has researched } \\
\text { HERS programs } \\
\text { - Stulte-level collaborative } \\
\text { developed policy } \\
\text { recommendations for } \\
\text { residential energy } \\
\text { efficiency (1992) } \\
\text { - MHAP (1991) } \\
\text { - REEMS (1991) }\end{array}$ & $\begin{array}{l}\text { - Good Cents: } \\
7 \text { utilities } \\
\text { - Super Good Cents: } \\
9 \text { utilities } \\
\text { - DSM programs: } \\
\text { S utilities } \\
\text { - MHAP (1991) } \\
\text { - REEMS (1991) }\end{array}$ & $\begin{array}{l}\text { - MHAP bomes } \\
\text { qualify for EEMs } \\
\text { through FHA } \\
\text {. REEMS peadiog } \\
\text { - } 1993 \text { legislation } \\
\text { requires MT Board } \\
\text { of Housiog to offer } \\
\text { EEMa of firt--ime } \\
\text { tromebuyers }\end{array}$ & REEMS pending & REEMS pending & $\begin{array}{l}\text { - Insures MHAP } \\
\text { mortgages } \\
\text { - REEMS pending }\end{array}$ & & & \\
\hline
\end{tabular}


Table 4. Summary of EEMs/HERS Activities by State (cont'd.)

\begin{tabular}{|c|c|c|c|c|c|c|c|c|c|}
\hline States & $\begin{array}{l}\text { Scaled HERS } \\
\text { Programs }\end{array}$ & $\begin{array}{l}\text { Utility } \\
\text { Programs }\end{array}$ & $\begin{array}{l}\text { Linkage with } \\
\text { EEMS }\end{array}$ & $\begin{array}{l}\text { Fannie } \\
\text { Mae }\end{array}$ & $\begin{array}{l}\text { Freddie } \\
\text { Mac }\end{array}$ & FHA/HUD & $\begin{array}{l}\text { Pilot } \\
\text { Tests }\end{array}$ & EPA & FmHA \\
\hline Nebraska & $\begin{array}{l}\text { SEO formed a task force to } \\
\text { deveiop HERS/EEMs policy } \\
\text { (1991) }\end{array}$ & $\begin{array}{l}\text { DSM programs: } \\
7 \text { ucilities }\end{array}$ & $\begin{array}{l}\text { Task force recom- } \\
\text { mended HERS/EEMs } \\
\text { linkage to governor }\end{array}$ & & & & & & \\
\hline Nevadiz & SEO is developing a HERS & $\begin{array}{l}\text { - Good Cents: } \\
2 \text { ualibies } \\
\text { - Super Good Cents: } \\
\text { 1 utibity } \\
\text { - DSM programs: } \\
3 \text { utilitios } \\
\end{array}$ & $\begin{array}{l}\text { SEO is considering } \\
\text { HERS/EEMs linkage }\end{array}$ & & & & & & \\
\hline $\begin{array}{l}\text { New } \\
\text { Hempethire }\end{array}$ & & $\begin{array}{l}\text { - DSM programs: } \\
2 \text { unilities } \\
\text { - Energy Crafied } \\
\text { Howes of New } \\
\text { England (1990) } \\
\text { (certification } \\
\text { program) }\end{array}$ & & & & & & & \\
\hline New Jersey & $\begin{array}{l}\text { SEO is convening ustk force } \\
\text { to develop HERS/EEMs } \\
\text { (1993) }\end{array}$ & $\begin{array}{l}\text { - Good Cents: } \\
2 \text { utilities } \\
\text { - DSM programs: } \\
6 \text { utilities } \\
\text { - Home Energy } \\
\text { Savings Program: } \\
7 \text { uilitices }\end{array}$ & $\begin{array}{l}\text { - SEO is convening } \\
\text { uask force to } \\
\text { develop } \\
\text { HERS/EEMs link } \\
\text { - SEO has } \\
\text { apposched NJ } \\
\text { Housing Finance } \\
\text { Agency, Fannie } \\
\text { Mare, and Freddie } \\
\text { Mac about EEMs }\end{array}$ & $\begin{array}{l}\text { Acceptance of } \\
\text { EEMs linked with } \\
\text { urility DSM } \\
\text { programs pending }\end{array}$ & $\begin{array}{l}\text { Acceptance of EEMs } \\
\text { linked with uility } \\
\text { DSM programs } \\
\text { pending }\end{array}$ & & & & \\
\hline New Mexico & $\begin{array}{l}\text { El Paso Electric Co. in Las } \\
\text { Cruces (Discovery Home) } \\
\text { (using ERHA }{ }^{17} \text { sofwere) }\end{array}$ & $\begin{array}{l}\text { DSM progtrams: } \\
\text { \& utibios }\end{array}$ & $\begin{array}{l}\text { EEMs available } \\
\text { through at least } 2 \text { Las } \\
\text { Cruces leaders }\end{array}$ & $\begin{array}{l}\text { Aocepts ERHA } \\
\text { racings for EEMs }\end{array}$ & $\begin{array}{l}\text { Accepts ERHA } \\
\text { ratings for EEMs }\end{array}$ & $\begin{array}{l}\text { Accepts ERHA ratings } \\
\text { for EEMs }\end{array}$ & & & $\begin{array}{l}\text { Stale building code } \\
\text { meetsjexcoeds } \\
\text { CABO MEC } 89\end{array}$ \\
\hline
\end{tabular}


Table 4. Summary of EEMs/HERS Activities by State (cont'd.)

\begin{tabular}{|c|c|c|c|c|c|c|c|c|c|}
\hline States & $\begin{array}{c}\text { Scaled HERS } \\
\text { Programs }\end{array}$ & $\begin{array}{l}\text { Utility } \\
\text { Programs }\end{array}$ & $\begin{array}{l}\text { Linkage with } \\
\text { EEMS }\end{array}$ & $\begin{array}{c}\text { Fannie } \\
\text { Mae }\end{array}$ & $\begin{array}{l}\text { Freddie } \\
\text { Mac }\end{array}$ & FHA/HUD & $\begin{array}{l}\text { Pilot } \\
\text { Tests }\end{array}$ & EPA & FmHA \\
\hline New York & $\begin{array}{l}\text { DOE-NYSO }{ }^{18} \text { plans to } \\
\text { research HERS software for } \\
\text { HERS/EEMs bousing } \\
\text { affordability pilot program }\end{array}$ & $\begin{array}{l}\text { NYSE-Star } 19 \\
\text { certification by } \\
\text { builders for new } \\
\text { homes } \\
\text { - Good Cents: } \\
1 \text { utility } \\
\text { DSM programs: } \\
12 \text { urilities } \\
\end{array}$ & $\begin{array}{l}\text { - NYSE-Star involv- } \\
\text { ing NY lenders in } \\
\text { EEMs; } 2 \text { active } \\
\text { - New York State } \\
\text { Mortgage Asso- } \\
\text { ciation guarantees } \\
\text { EEMs for NYSO } \\
\text { pilot program }\end{array}$ & $\begin{array}{l}\text { AocepLs NYSE-Star } \\
\text { for EEMs }\end{array}$ & & & $\begin{array}{l}\text { DOE-NYSO pian- } \\
\text { ning HERS/EEMs } \\
\text { housing afford- } \\
\text { abiliny project in } \\
\text { Whute Plains, NY. } \\
\text { and Newark. NJ }\end{array}$ & & $\begin{array}{l}\text { State building conde } \\
\text { meetsiexceeds } \\
\text { CABO MEC } 89\end{array}$ \\
\hline $\begin{array}{l}\text { North } \\
\text { Carolina }\end{array}$ & $\begin{array}{l}\text { - SEO formed advisory } \\
\text { comminee to develop } \\
\text { HERS (1993) } \\
\text { - NC Allernative Energy } \\
\text { Corp. developing HERS } \\
\text { for builders (Exemplary } \\
\text { Home Progrum) (1991) }\end{array}$ & $\begin{array}{l}\text { DSM programs: } \\
11 \text { utilivies } \\
\text { - Max Energy Effi-. } \\
\text { cient Homes (1988) } \\
\text { (cerificaion } \\
\text { program): } \\
1 \text { uility }\end{array}$ & $\begin{array}{l}\text { - SEO negotiating } \\
\text { with lenders to } \\
\text { offer EEMsi; } 1 \text { bank } \\
\text { ocive } \\
\text { - NC Allermative } \\
\text { Energy Corp. } \\
\text { planning EEMs } \\
\text { linkage (1991) } \\
\text { - GMAC } \\
\text { acceptance of Max } \\
\text { E-E Homes pro- } \\
\text { grem for EEMs } \\
\text { pending } \\
\end{array}$ & $\begin{array}{l}\text { - Exploring NC } \\
\text { EEMs pilot } \\
\text { - Aoceptance of } \\
\text { Max Energy } \\
\text { Efficient Home } \\
\text { Program for } \\
\text { EEMs pending }\end{array}$ & $\begin{array}{l}\text { Acceptance of Max } \\
\text { Energy Efficient } \\
\text { Home Program for } \\
\text { EEMs peading }\end{array}$ & & $\begin{array}{l}\text { Fannie Mae } \\
\text { exploring NC } \\
\text { EEMs pilor }\end{array}$ & & \\
\hline North Dakota & $\begin{array}{l}\text { SEO interested in } \\
\text { developing HERS }\end{array}$ & $\begin{array}{l}\text { DSM programs: } \\
7 \text { utilities }\end{array}$ & & & & & & & \\
\hline Ohio & $\begin{array}{l}\text { SEO formed collaborative to } \\
\text { develop HERS/EEMs }\end{array}$ & $\begin{array}{l}\text { - Good Cents: } \\
1 \text { utility } \\
\text { - DSM programs: } \\
8 \text { utilities }\end{array}$ & $\begin{array}{l}\text { - Collaborative } \\
\text { developing EEMs } \\
\text { linkage } \\
\text { - } 1 \text { bank active in } \\
\text { EEMs }\end{array}$ & & & & & & \\
\hline Otclabome & & $\begin{array}{l}\text { - Good Cents: } \\
3 \text { utilities } \\
\text { - DSM progrems: } \\
6 \text { uditicies }\end{array}$ & $\begin{array}{l}1 \text { bank active in } \\
\text { EEMs }\end{array}$ & & & & & & \\
\hline
\end{tabular}


Table 4. Summary of EEMs/HERS Activities by State (cont'd.)

\begin{tabular}{|c|c|c|c|c|c|c|c|c|c|}
\hline States & $\begin{array}{l}\text { Scaled HERS } \\
\text { Programs }\end{array}$ & $\begin{array}{l}\text { Utility } \\
\text { Programs }\end{array}$ & $\begin{array}{l}\text { Linkage with } \\
\text { EEMS }\end{array}$ & $\begin{array}{c}\text { Fannie } \\
\text { Mae }\end{array}$ & $\begin{array}{l}\text { Freddie } \\
\text { Mac }\end{array}$ & FHAVHUD & $\begin{array}{l}\text { Pilot } \\
\text { Tests }\end{array}$ & EP'A & FmHA \\
\hline Oregon & $\begin{array}{l}\text { - SEO is developing a } \\
\text { statewide HERS } \\
\text { - MHAP (1991) } \\
\text { - REEMS (1991) }\end{array}$ & $\begin{array}{l}\text { - Good Cents: } \\
36 \text { utilities } \\
\text { - Super Cood Cents: } \\
33 \text { urilities } \\
\text { - DSM programs: } \\
11 \text { utilities } \\
\text { - MHAP (1991) } \\
\text { - REEMS (1991) }\end{array}$ & $\begin{array}{l}\text { - SEO working with } \\
\text { AHFC } 21 \text { on } \\
\text { EEMs linkage with } \\
\text { real estale agents, } \\
\text { lenders, and } \\
\text { - apprasers } \\
\text { 11 lending insiitu- } \\
\text { cions acive in } \\
\text { EEMs } \\
\text { - MHAP homes } \\
\text { qualify for EEMs } \\
\text { through FHA } \\
\text { - REEMS pending }\end{array}$ & $\begin{array}{l}\text { - Accepts OR } \\
\text { energy code for } \\
\text { EEMs } \\
\text { - REEMS pending }\end{array}$ & $\begin{array}{l}\text { - Accepts OR } \\
\text { energy code for } \\
\text { EEMs } \\
\text { - REEMS pending }\end{array}$ & $\begin{array}{l}\text { - Accepts OR energy } \\
\text { code for EEMs } \\
\text { - Insures MHAP } \\
\text { mortgages } \\
\text { - REEMS pending }\end{array}$ & & & $\begin{array}{l}\text { State building code } \\
\text { meets/exceeds } \\
\text { CABO MEC } 89\end{array}$ \\
\hline Peansylvania & SEO is interested in HERS & $\begin{array}{l}\text { - Good Cents: } \\
3 \text { uditives } \\
\text { - DSM programs: } \\
9 \text { udilities } \\
\text { - Philedelphia Elociric } \\
\text { Co. (Excellence in } \\
\text { Energy Efficiency) } \\
\text { (1985) (certification } \\
\text { program) }\end{array}$ & $\begin{array}{l}\text { SEO is interested in } \\
\text { HERS/EEMS linkage }\end{array}$ & & & & & & \\
\hline Puerto Rico & $\begin{array}{l}\text { DOCA } 22 \text { is interested in } \\
\text { HERS/EEMs }\end{array}$ & & $\begin{array}{l}\text { DOCA is interested in } \\
\text { HERS/EEMs }\end{array}$ & & & & & & \\
\hline Rhode Isiand & $\begin{array}{l}\text { RISE }^{23} \text { (1989) (uses } \\
\text { ERHA software) }\end{array}$ & $\begin{array}{l}\text { - DSM programs: } \\
2 \text { utilities } \\
\text { - Energy Crafied } \\
\text { Homes of New } \\
\text { Englund (1990) } \\
\text { (cartification } \\
\text { program) }\end{array}$ & $\begin{array}{l}\text { EEMs through RI } \\
\text { Housing and } \\
\text { Mongage Finance } \\
\text { Corporation }\end{array}$ & $\begin{array}{l}\text { Accepts RISE for } \\
\text { EEMs }\end{array}$ & $\begin{array}{l}\text { Accepts RISE for } \\
\text { EEMs }\end{array}$ & $\begin{array}{l}\text { Accepts RISE for } \\
\text { EEMs }\end{array}$ & & & $\begin{array}{l}\text { Statc building code } \\
\text { meetsexceeds } \\
\text { CABO MEC } 89\end{array}$ \\
\hline
\end{tabular}


Table 4. Summary of EEMs/HERS Activities by State (cont'd.)

\begin{tabular}{|c|c|c|c|c|c|c|c|c|c|}
\hline States & $\begin{array}{l}\text { Scaled HERS } \\
\text { Programs }\end{array}$ & $\begin{array}{l}\text { Utility } \\
\text { Programs }\end{array}$ & $\begin{array}{l}\text { Linkage with } \\
\text { EEMS }\end{array}$ & $\begin{array}{l}\text { Fannie } \\
\text { Mae }\end{array}$ & $\begin{array}{l}\text { Freddie } \\
\text { Mac }\end{array}$ & FHAMHUD & $\begin{array}{l}\text { Pilot } \\
\text { Tests }\end{array}$ & EPA & FmHA \\
\hline $\begin{array}{l}\text { South } \\
\text { Carolina }\end{array}$ & & $\begin{array}{l}\text { - Good Cents: } \\
19 \text { utilities } \\
\text { - DSM programs: } \\
5 \text { utilities } \\
\end{array}$ & $\begin{array}{l}\text { SEO is interested in } \\
\text { developing HERS } \\
\text { EEMs }\end{array}$ & & & & & & $\begin{array}{l}\text { Stace building code } \\
\text { meets/exceeds } \\
\text { CABO MEC } 89\end{array}$ \\
\hline South Dakota & $\begin{array}{l}\text { SEO is incerested in } \\
\text { developing a HERS }\end{array}$ & $\begin{array}{l}\text { - DSM programs: } \\
8 \text { urilities } \\
\text { - SDHBA }{ }^{24} \text { is } \\
\text { developiug a } \\
\text { certification } \\
\text { program for now } \\
\text { bomes (Cost } \\
\text { Affordable } \\
\text { Staodards for } \\
\text { Homes) }\end{array}$ & & & & & & & \\
\hline Tennessee & & $\begin{array}{l}\text { DSM programs: } \\
4 \text { ucilities }\end{array}$ & & & & & & & \\
\hline Texes & $\begin{array}{l}\text { - Austin Energy Star (City } \\
\text { of Austin) (1985) (uses } \\
\text { BETA software) } \\
\text { - HERS pilot project, } \\
\text { University of Texas at } \\
\text { El Paso (1990) (uses } \\
\text { mouified ERHA software) }\end{array}$ & $\begin{array}{l}\text { - Good Cents: } \\
24 \text { utilities } \\
\text { DSM programs: } \\
19 \text { utilities } \\
\text { - West TX Utility Co. } \\
\text { (Energy Saving Plan } \\
\text { Award) (1983) (cer- } \\
\text { ificacion program) }\end{array}$ & $\begin{array}{l}\text { - TX-EEs } 26 \text { pro- } \\
\text { vides technical } \\
\text { assistunce on EEMs } \\
\text { to builders, lenders, } \\
\text { trade associations, } \\
\text { and utilities } \\
\text { - Austin Energy Star } \\
\text { program consider- } \\
\text { ing linkage with } \\
\text { EEMs } \\
\text { - Ubility in west } \\
\text { Texas contacting } \\
\text { lenders about } \\
\text { EEMs }\end{array}$ & & & $\begin{array}{l}\text { TX-EES working with } \\
\text { HUD on adapting } \\
\text { EEMs guidelines to } \\
\text { local climate }\end{array}$ & $\begin{array}{l}\text { HERS pilot in } \\
\text { El Paso }\end{array}$ & & \\
\hline
\end{tabular}


Table 4. Summary of EEMs/HERS Activities by State (cont'd.)

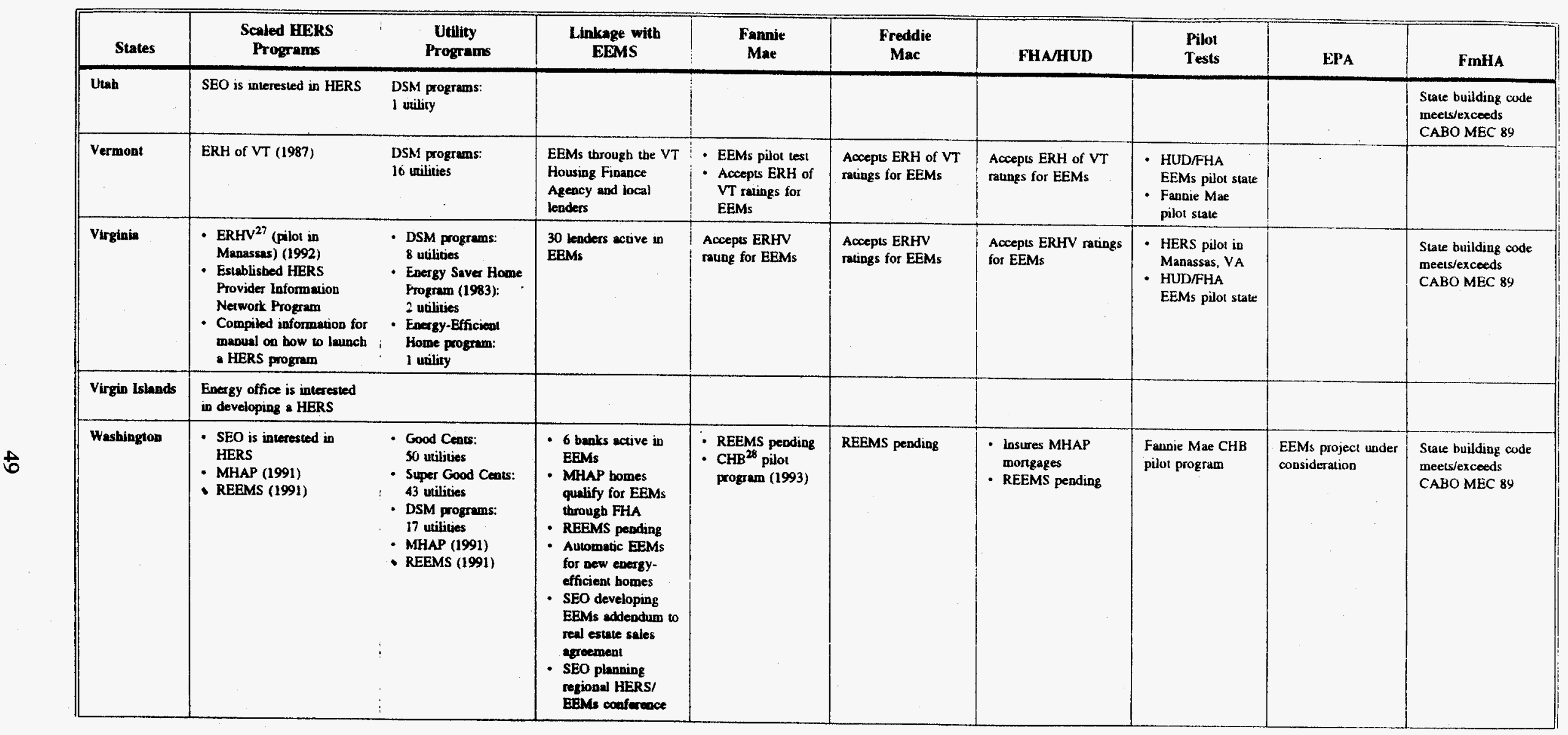




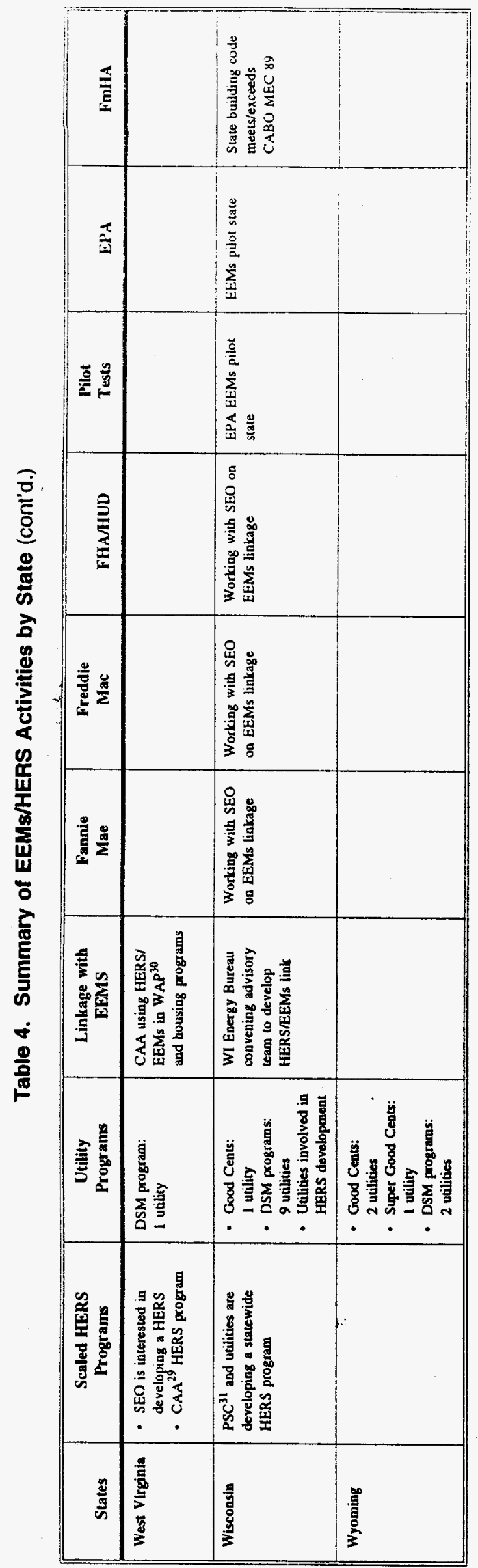

TP-5478

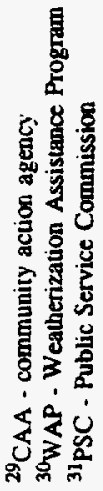


Table 5. Actions by National-level HERS/EEMs Stakeholder Groups, Spring 1992-Spring 19931

\begin{tabular}{|c|c|c|c|c|c|c|}
\hline $\begin{array}{l}\text { Stakeholder } \\
\text { group }\end{array}$ & $\begin{array}{l}\text { Building } \\
\text { awareness }\end{array}$ & $\begin{array}{c}\text { HERS/EEMs } \\
\text { network } \\
\text { participation }\end{array}$ & $\begin{array}{l}\text { Proactive } \\
\text { initiatives }\end{array}$ & $\begin{array}{c}\text { Reviews and } \\
\text { changes in } \\
\text { policies/practices }\end{array}$ & $\begin{array}{l}\text { Pilot } \\
\text { testing }\end{array}$ & Studies \\
\hline $\begin{array}{l}\text { Consumer gro } \\
\text { AARP }\end{array}$ & & & & $\begin{array}{l}\text { - } 1992 \text { Public Policy } \\
\text { Agenda position in } \\
\text { HERS/EEMs } \\
\text { - Continuing review } \\
\end{array}$ & & \\
\hline CFA & $\begin{array}{l}\text { Held public forum in } \\
1992 \text { to discuss A Blue- } \\
\text { print for Action }\end{array}$ & $\begin{array}{l}\text { HERS Council planning } \\
\text { committee, member, } \\
\text { Board of Directors }\end{array}$ & & & & $\begin{array}{l}\text { P'repared paper on } \\
\text { real estate issues in } \\
\text { HERS/EEMs } \\
\text { Iransactions } \\
\end{array}$ \\
\hline $\begin{array}{l}\text { Home builders } \\
\text { EEBA }\end{array}$ & & & $\begin{array}{l}\text { Developing guidelines } \\
\text { for energy-efficient } \\
\text { home construction }\end{array}$ & & & \\
\hline NAHB & $\begin{array}{l}\text { - Published articles on } \\
\text { EEMs in NAHB } \\
\text { newsletter } \\
\text { - Had a session on } \\
\text { HERS/EriMs at } 1993 \\
\text { convention }\end{array}$ & $\begin{array}{l}\text { - HERS Council } \\
\text { planning committee, } \\
\text { member, Board of } \\
\text { Directors } \\
\text { - Served as resource to } \\
\text { HUD Task Force }\end{array}$ & $\begin{array}{l}\text { Developed code } \\
\text { compliance document } \\
\text { for builders }\end{array}$ & $\begin{array}{l}\text { Passed resolutions in } \\
1991 \text { and } 1992 \\
\text { endorsing lEEMs and } \\
\text { covering other related } \\
\text { matters }\end{array}$ & & \\
\hline
\end{tabular}

\footnotetext{
'Appendix B presents details. Organizations listed were members of the HERS/EEMs National Collaborative during 1991 and 1992.
} 
Table 5. Actions by National-level HERS/EEMs Stakehölder Groups, Spring 1992-Spring 1933 (Cont'd.)

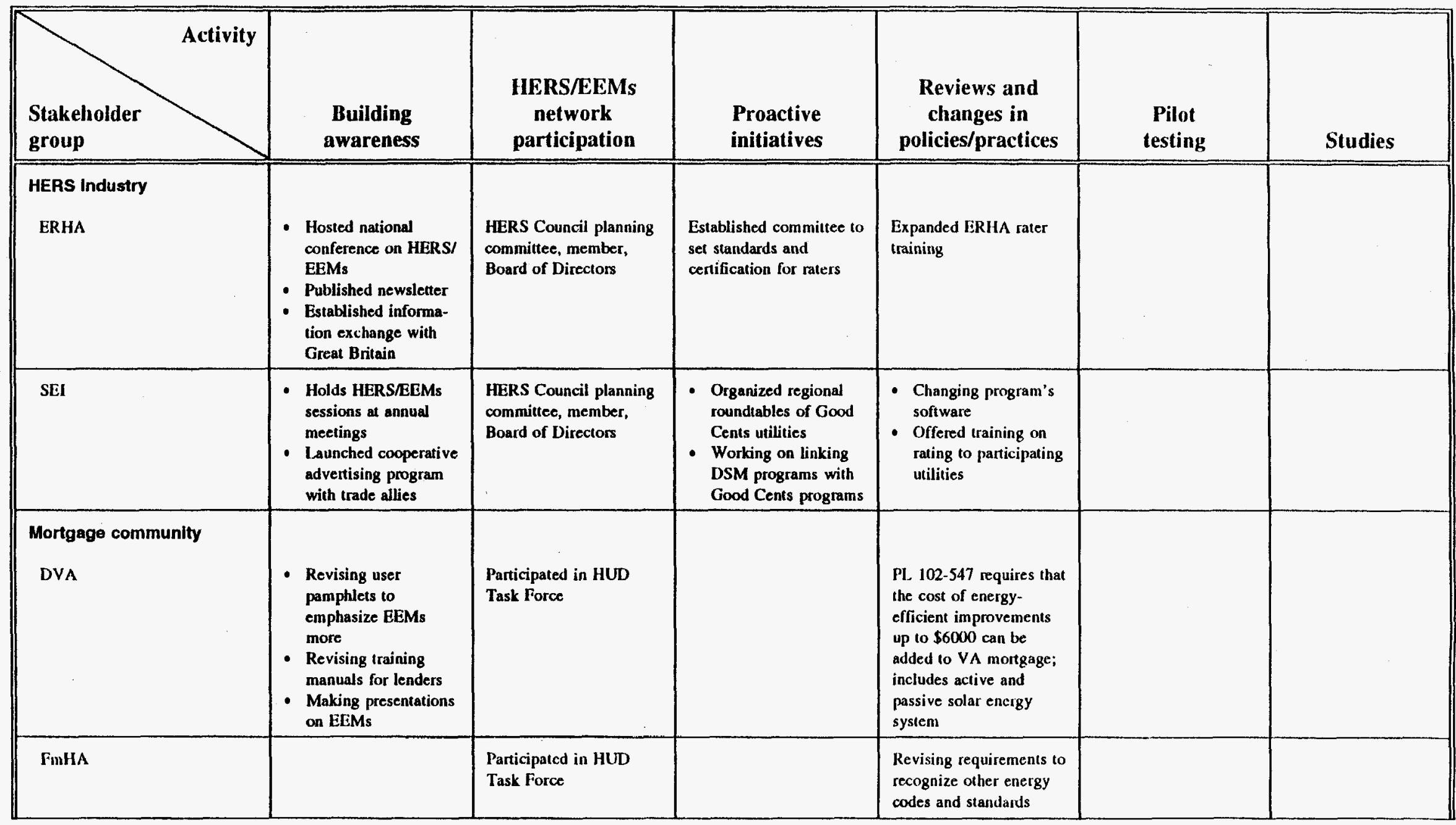


Table 5. Actions by National-level HERS/EEMs Stakeholder Groups, Spring 1992-Spring 1933 (Cont'd.)

\begin{tabular}{|c|c|c|c|c|c|c|}
\hline $\begin{array}{l}\text { Stakeholder } \\
\text { group }\end{array}$ & $\begin{array}{c}\text { Building } \\
\text { awareness }\end{array}$ & $\begin{array}{c}\text { HERS/EEMs } \\
\text { network } \\
\text { participation }\end{array}$ & $\begin{array}{l}\text { Proactive } \\
\text { initiatives }\end{array}$ & $\begin{array}{c}\text { Reviews and } \\
\text { changes in } \\
\text { policies/practices }\end{array}$ & $\begin{array}{l}\text { Pilot } \\
\text { testing }\end{array}$ & Studies \\
\hline $\begin{array}{l}\text { Mortgage community } \\
\text { (cont'd.) } \\
\text { Freddie Mac (FHLMC) }\end{array}$ & $\begin{array}{l}\text { Granted interviews to } \\
\text { the media, written up as } \\
\text { articles }\end{array}$ & $\begin{array}{l}\text { Participated in the HUD } \\
\text { Task Force }\end{array}$ & & & & $\begin{array}{l}\text { Analyzed Freddic } \\
\text { Mac mortgage data } \\
\text { for ERHA and ERH } \\
\text { of VT-rated homes } \\
\text { with inconclusive } \\
\text { results on default } \\
\text { rates }\end{array}$ \\
\hline HUID/FHA & $\begin{array}{l}\text { Trained lenders in EEMs } \\
\text { concepts }\end{array}$ & & $\begin{array}{l}\text { Chaired the HUD } \\
\text { Task Force } \\
\text { (Affordable Housing } \\
\text { Act of 1990) } \\
\text { Will issue the Task } \\
\text { Force recommenda- } \\
\text { tions when signed }\end{array}$ & & $\begin{array}{l}\text { - Under EPACT and } \\
\text { the Housing Act of } \\
1992, \text { will run pilot } \\
\text { tests in } 5 \text { states on } \\
\text { ELiMs in } 1993 \text { and } \\
1994 \\
\text { - EEMs concepts re- } \\
\text { commended by the } \\
\text { HUD Task Force } \\
\text { may be pilot tested } \\
\text { in states } \\
\end{array}$ & \\
\hline Fannie Mae (FNMA) & & $\begin{array}{l}\text { - HERS Council Board } \\
\text { of Directors } \\
\text { - Participated in HUD } \\
\text { Task Force }\end{array}$ & $\begin{array}{l}\text { Working to develop new } \\
\text { lending initiatives with } \\
\text { utilities }\end{array}$ & $\begin{array}{l}\text { - Instituted 2\% ratio } \\
\text { stretch } \\
\text { - Are reviewing current } \\
\text { policies relative to } \\
\text { EEMs }\end{array}$ & $\begin{array}{l}\text { - Washington State } \\
3 / 2 \text { option pilot } \\
\text { program and other } \\
\text { piloss } \\
\text { - VT Housing } \\
\text { Finance Agency } \\
\text { pilot program } \\
\text { - Southern Maryland } \\
\text { Electric Cooperative } \\
\text { pilot program }\end{array}$ & $\begin{array}{l}\text { Analyzed default } \\
\text { rates of FNMA } \\
\text { EEMS on LRHA- } \\
\text { rated homes with } \\
\text { inconclusive results }\end{array}$ \\
\hline MBA & $\begin{array}{l}\text { Published articles in } \\
\text { their newsletter }\end{array}$ & $\begin{array}{l}\text { HERS Council Board of } \\
\text { Directors }\end{array}$ & & & & \\
\hline
\end{tabular}


Table 5. Actions by National-level HERS/EEMs Stakeholder Groups, Spring 1992-Spring 1933 (Cont'd.)

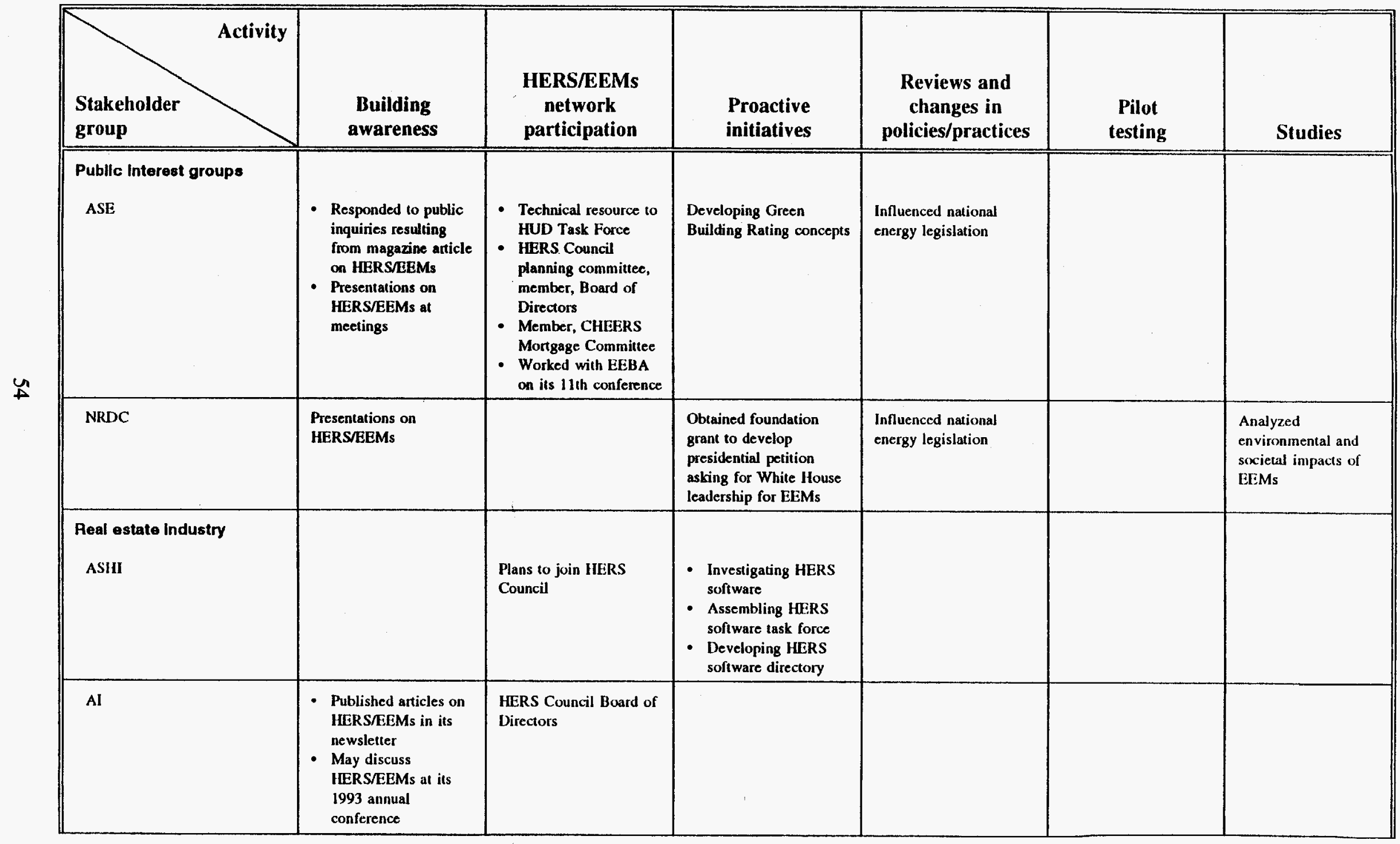


Table 5. Actions by National-level HERS/EEMs Stakeholder Groups, Spring 1992-Spring 1933 (Cont'd.)

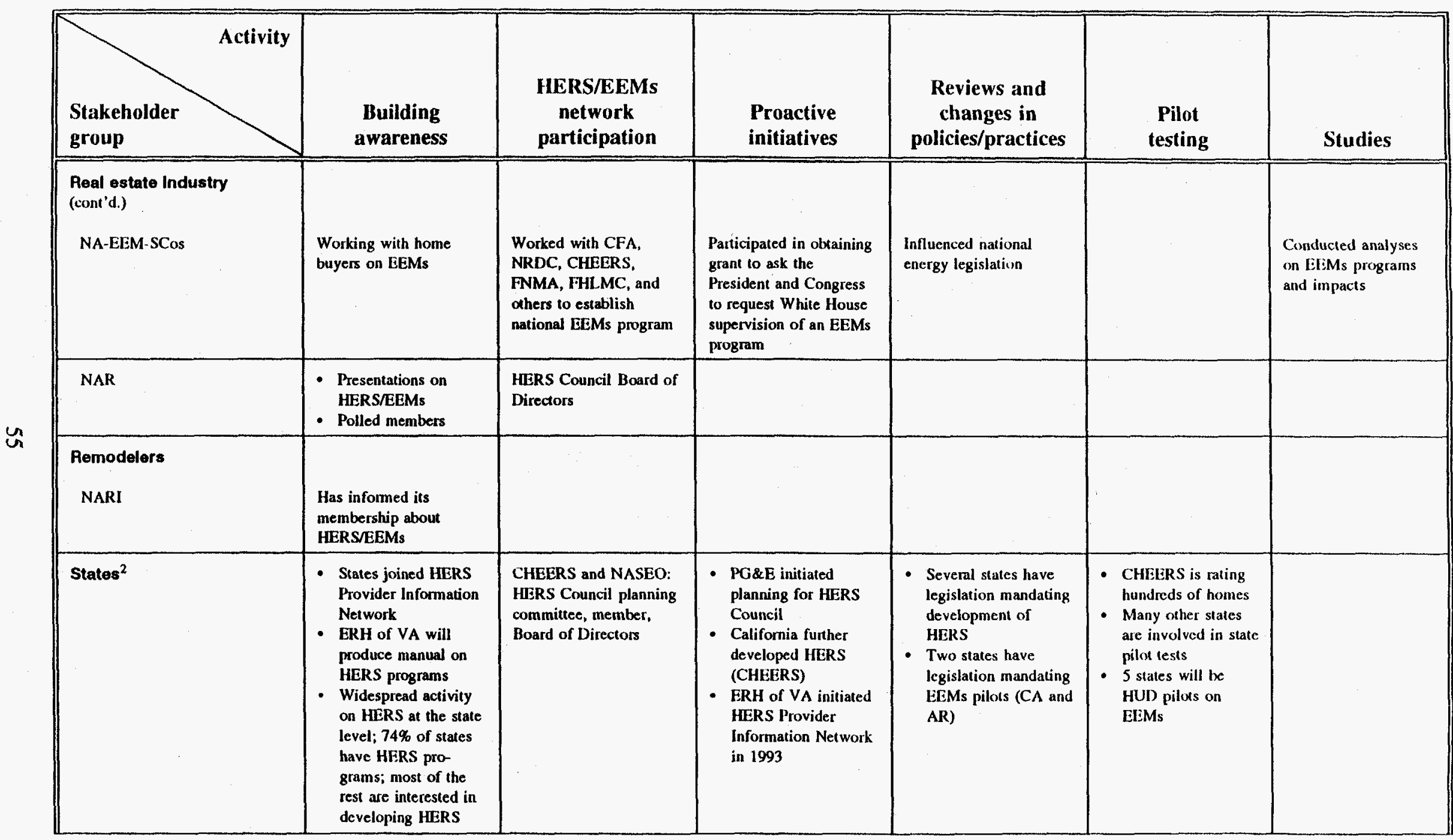

${ }^{2}$ See Table 2 and Appendix A for details on state activities. 
Table 5. Actions by National-level HERS/EEMs Stakeholder Groups, Spring 1992-Spring 1933 (Cont'd.) $\ldots \ldots$...

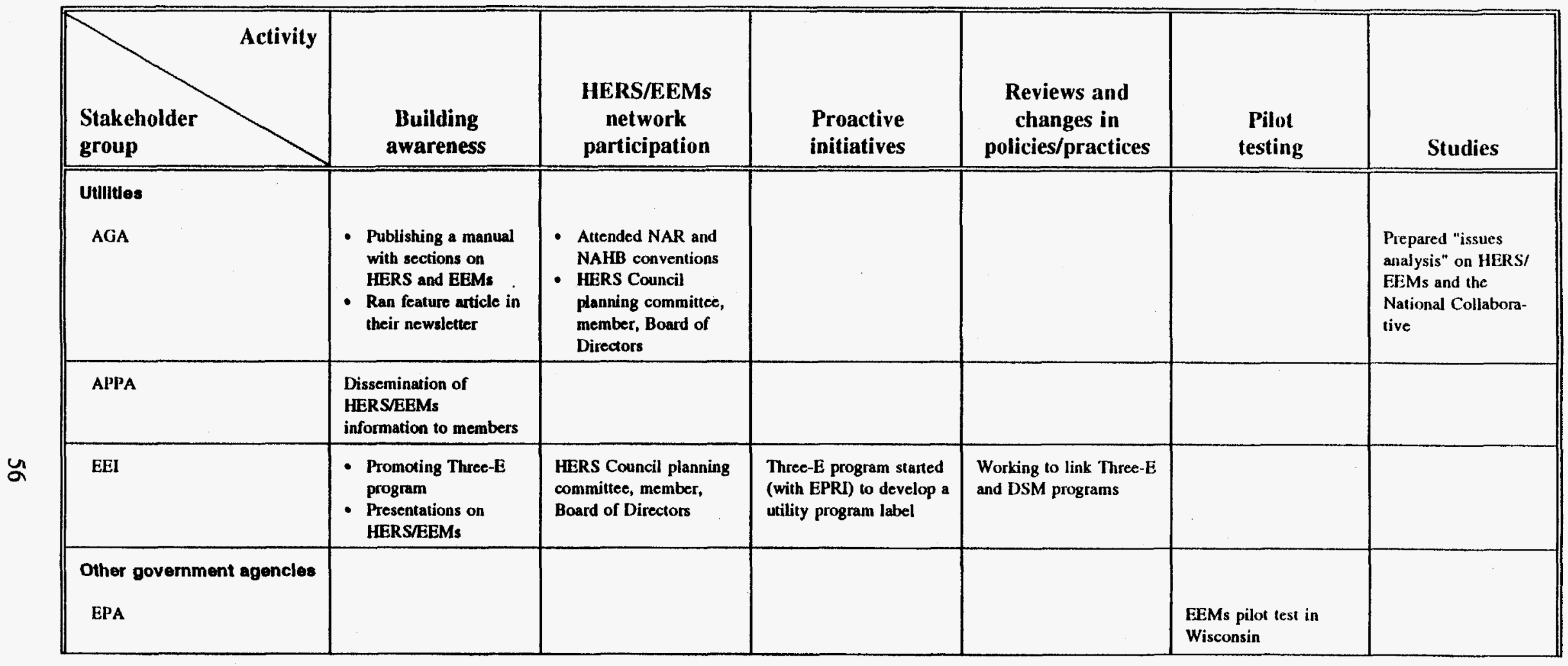


Table 5. Actions by National-level HERS/EEMs Stakeholder Groups, Spring 1992-Spring 1933 (Cont'd.)

\begin{tabular}{|c|c|c|c|c|c|c|}
\hline $\begin{array}{l}\text { Stakeholder } \\
\text { group }\end{array}$ & $\begin{array}{c}\text { Building } \\
\text { awareness }\end{array}$ & $\begin{array}{l}\text { HERS/EEMs } \\
\text { network } \\
\text { participation }\end{array}$ & $\begin{array}{l}\text { Proactive } \\
\text { initiatives }\end{array}$ & $\begin{array}{c}\text { Reviews and } \\
\text { changes in } \\
\text { policies/practices }\end{array}$ & $\begin{array}{c}\text { Pilot } \\
\text { testing }\end{array}$ & Studies \\
\hline DOE/NREL & $\begin{array}{l}\text { Dissemination of } \\
\text { Blueprint for Action, } \\
\text { Going National with } \\
\text { HERS and EEMs: } \\
\text { The Collected Papers } \\
\text { of the National } \\
\text { Collaborative and } \\
\text { aher docuenents } \\
\text { - Press releases, } \\
\text { interviews with media } \\
\text { - Nunerous } \\
\text { presentations at } \\
\text { meetings }\end{array}$ & $\begin{array}{l}\text { - Supporting } \\
\text { development of } \\
\text { HERS Council } \\
\text { - Convened interagency } \\
\text { management team } \\
\text { - Participated in HUD } \\
\text { Task Force }\end{array}$ & $\begin{array}{l}\text { - Initiated and } \\
\text { facilitated National } \\
\text { HERS/EEMs } \\
\text { Collaborative } \\
\text { - Developing HERS } \\
\text { tool certification } \\
\text { protocols and } \\
\text { guidelines with the } \\
\text { HERS Council } \\
\text { - Provided technical } \\
\text { expertise on energy- } \\
\text { efficiency and solar } \\
\text { energy in buildings } \\
\text { - Funded a regional } \\
\text { HERSEEMs collab- } \\
\text { orative in the Pacific } \\
\text { Northwest }\end{array}$ & $\begin{array}{l}\text { - Developing, with the } \\
\text { HliRS Cinncil, } \\
\text { guidelines under } \\
\text { EPAct } \\
\text { - Developing teport to } \\
\text { Congress under } \\
\text { EPAd }\end{array}$ & $\begin{array}{l}\text { - Supponting HUD in } \\
\text { El:Ms pilor tests } \\
\text { - Developing EIEM- } \\
\text { DSM linkages, } \\
\text { working with utility } \\
\text { regulators and } \\
\text { utilities }\end{array}$ & $\begin{array}{l}\text { Monitored and } \\
\text { reported develop- } \\
\text { ments in the nation } \\
\text { and assessed progress }\end{array}$ \\
\hline
\end{tabular}




\section{Bibliography}

Barakat and Chamberlin, Inc. 1992. Energy-Efficiency Resource Directory: A Guide to Utility Programs. 2nd Printing. Prepared for the President's Commission on Environmental Quality. Washington, D.C. December.

Battelle Pacific Northwest Laboratory. 1993. "OBT Data Crib Sheets," Washington, D.C., May.

Bonneville Power Administration. 1993. Residential Conservation Agreement: Participating Utilities, Portland, Oregon: Bonneville Power Administration. February 25.

Burmeister, George. 1993. Home Energy Rating Systems State Legislative Report (Rough Draft). Washington, D.C.: National Council of State Legislatures. September.

Cohen, Rebecca Arnold. 1993. Addressing the Concerns of Real Estate Professionals about Their Role in Promoting Home Energy Rating Systems and Energy Efficient Mortgages. Prepared for the National Renewable Energy Laboratory. Washington D.C.: Regulatory Alternatives Development Corporation. January.

Faesy Richard. 1991. "Home Energy Ratings Gain Increasing Support among State and Federal Legislators." The Energy Efficient Mortgage Newsletter, 4:1. Fall.

Farmers Home Administration. 1993. Correspondence from Rich Davis and Samuel J. Hodges, III to Barbara Farhar and Jan Eckert (NREL) concerning FmHA list of states meeting the CABO MEC 1989 building code.

Governor's Energy Task Force. 1993. Strategic Energy Plan for Maryland: Recommendations of the Governor's Energy Task Force. Annapolis, MD: Maryland Energy Administration.

Hughes, Ron. 1992. List of State Activities in Selected States. Little Rock, AR: Energy Rated Homes of America. October.

Kuntz, Gail. 1992. Memorandum to John Elizalde re: Request to Federal Home Administration to raise loan cap for EEMs in Montana. Bonneville Power Administration. September 29.

Maize, Kennedy and John McCaughey. 1993. "DSM at Mid-Passage: A Discussion of the State of the Art and Science of Demand-Side Management in Electric Utilities." The Quad Report. Washington, D.C.: Consumer Energy Council of America Research Foundation. Spring.

Mortgage Bankers Association of America. 1993. "1-4 Family Mortgage Originations." Washington, D.C. August 2.

National Association of State Energy Officials. 1991. NASEO Survey on Home Energy Rating Systems. Washington, D.C.: National Association of State Energy Officials.

National HERS/EEMs Collaborative. 1992a. A National Program for Energy-Efficient Mortgages and Home Energy Rating Systems: A Blueprint for Action. (NREL/TP-261-4677) Golden, CO: National Renewable Energy Laboratory. March. 
1992b. Going National with HERS and EEMs, Issues and Impacts: The Collected Papers of the National Collaborative. (NREL/TP-261-4706) Golden, CO: National Renewable Energy Laboratory. March.

1992c. Stakeholder Perceptions of a Potential National Program of Energy Rated Mortgages. Washington, D.C.: National Renewable Energy Laboratory. October 29.

National Renewable Energy Laboratory. 1992. Organizational Review Process, Comments, and Activities. for the HERS/EEMs National Collaborative. (Draft) Washington, D.C. September 30.

Princeton Economic Research, Inc. (PERI): 1991-1992. National HERS/EEMs Collaborative Draft Minutes. Rockville, MD.

1992. National HERS/EEMs Collaborative Draft Minutes: 11 th and 12th Meetings. Rockville, MD: June 12 and October 30.

Snead, Bruce. 1991. "Legislation Introduced for Home Energy Rating Systems." The National Energy Specialist Newsletter, p. 5. May.

Southern Electric International, Inc. 1992. Good Cents Utilities. (Flyer) March 1. Farhar (NREL).

Taylor, Christine. 1993. Memorandum to Barbara Farhar re: HERS Provider Information Network. Richmond, Virginia: Energy Rated Homes of Virginia. March 29.

U.S. Department of Energy, Office of Technical and Financial Assistance. 1992. Building Efficiency National Inventory. Washington, D.C.: November.

U.S. Department of Housing and Urban Development. 1992. "Uniform Plan for Energy Efficiency, Organizational Statement" (Draft). Washington, D.C. June 11.

Utility Data Institute. 1992. The Official Guide to Demand-Side Management Programs and Research. 2nd Edition, Washington, D.C.: Utility Data Institute. September.

Vories, Rebecca and Karen George. 1991. Analysis of Home Energy Rating Systems: Results of Brief Interviews. (DE-AC06-76RLO 1830) Denver, CO: Infinite Energy, Inc. for the Pacific Northwest Laboratory, Richland, WA. October. 
Appendix A

State Activities 
Introduction

Alabama

Alaska

A-7

Arizona

A- 12

Arkansas

California

Colorado

Connecticut

A-26

Delaware

A-28

District of Columbia

A-29

Florida

A-30

Georgia

A-33

Hawaii

A-36

Idaho

A-38

Illinois

A -42

Indiana

A- 45

Iowa

A-48

Kansas

A-51

Kentucky

A-53

Louisiana

A-55

Maine

A- 58

Maryland

A- 59

Massachusetts

A- 62

Michigan

A- 63

Minnesota

A. 65

Mississippi

A-68

Missouri

A-71

Montana

A- 72

Nebraska

A-75

Nevada

A-76

New Hampshire

A-77

New Jersey

A-78

New Mexico

A-80

New York

A-82

North Carolina

A-87

North Dakota

A- 90

Ohio

A-91

Oklahoma

A-95

Oregon . .

A-97

Pennsylvania

A-102

Puerto Rico

A-104

Rhode Island

A-105 
Contents (Continued)

$\underline{\text { Page }}$

South Carolina

A-107

South Dakota

A-109

Tennessee

A-111

Texas

A- 112

Utah . .

A-115

Vermont

A-116

Virginia

A- 120

Virgin Islands

A- 124

Washington

A- 125

West Virginia

A-131

Wisconsin

A-132

Wyoming

A- 135 


\section{Introduction}

Appendix A assembles, by state, primary and secondary information on HERS and EEMs programs in 50 states, the District of Columbia, Puerto Rico, and the Virgin Islands. The appendix documents the data in Table 1. Primary information was derived from focused telephone interviews with state energy officials, program managers, and others involved with HERS and HERS/EEM programs at the state level. Secondary information was drawn from recent studies, surveys, lists, national HERS/EEMs collaborative meeting minutes, and correspondence in NREL files. Information from these secondary sources was summarized by state. The secondary sources used were:

- U.S. Department of Energy, Office of Technical and Financial Assistance. 1992. Building Efficiency National Inventory. Washington, D.C. November.

- National Association of State Energy Officials (NASEO). 1991. NASEO Survey on Home Energy Rating Systems. Washington D.C.: National Association of State Energy Officials.

- $\quad$ Rebecca Vories and Karen George. 1991. Analysis of Home Energy Rating Systems: Results of Brief Interviews. (DE-AC06-76RLO0 1830) for Pacific Northwest Laboratory, Denver, CO: Infinite Energy. October.

- $\quad$ Southern Electric International, Inc. 1992. Good Cents Utilities (Flyer). March 1.

- Southern Electric International, Inc. 1993. Correspondence on Good Cents Utilities between Benny Folsom (SEI), and Barbara Farhar (NREL).

- Bonneville Power Administration. 1993. Residential Conservation Agreement: Participating Utilities. Portland, OR: Bonneville Power Administration. February 25.

- Barakat and Chamberlin, Inc. 1992. Energy-Efficiency Resource Directory: A Guide to Utility Programs. 2nd Printing. Prepared for the President's Commission on Environmental Quality. Washington, D.C. December.

- Utility Data Institute. 1992. The Official Guide to Demand-Side Management Programs and Research. 2nd edition. Washington, D.C.: Utility Data Institute. September.

- Christine Taylor. 1993. Memorandum to Barbara Farhar re: HERS Provider Information Network. Richmond, VA: Energy Rated Homes of Virginia. March 29.

- Princeton Economic Research Inc. (PERI). 1992. National HERS/EEMS Collaborative: Draft Minutes, 11th and 12th Meetings. Rockville, MD. June 12 and October 30.

- Ron Hughes. 1992. List of Activities in Selected States. Little Rock, AR: Energy Rated Homes of America. October.

- Farmers Home Administration. 1993. Correspondence from Rich Davis and Samuel J. Hodges, III (FmHA) to Barbara Farhar and Jan Eckert (NREL) concerning the FmHA list of states that have either adopted or accepted an energy code/standard that is comparable to, or more stringent than the FmHA thermal requirements for single family housing construction or $\mathrm{CABO}$ MEC 1989 building codes. 
Information on utility DSM programs comes from Barakat and Chamberlin (1992) and the Utility Data Institute (1992). The latter source is used for utility DSM programs not mentioned in the former.

Of the 53 states and territories included in Appendix A, 36 states were selected for in-depth interviews conducted between January and April 1993. Included are three groups of states:

1. States that either had HERS/EEM linkages in place or were developing linkages, based on a review of secondary sources. Nine states fell into this category: Alaska, California, Indiana, Iowa, Mississippi, New Mexico, Ohio, South Dakota, and Vermont.

2. States for which inconsistent secondary information existed. Some sources identified several states as having no HERS programs while other sources reported that HERS programs were in place or under development in those states. These discrepancies in information may be attributed to differences among studies in time of data collection, methods used for collecting data, and some informants lack of familiarity with HERS and EEMs. The situation in 17 states required further clarification-Colorado, Connecticut, Florida, Georgia, Hawaii, Mlinois, Louisiana, Maryland, Minnesota, New Jersey, Oklahoma, West Virginia, Rhode Island, South Carolina, South Dakota, Tennessee, and Utah.

3. States that had HERS programs in place, were developing HERS, or were running pilot programs based on NREL's current knowledge. These states included Arizona, Arkansas, Kansas, Michigan, New York, Oregon, and Virginia.

It was beyond the scope of the present effort to collect primary information on all states and territories included in this appendix.

For each state entry, NREL analysts interviewed one to five individuals, often more than once, to clarify information. Informants had the opportunity to review and edit the drafts on their states. The interviews covered four subject areas:

- The background and current status of HERS and EEMs activities

- Why these activities were pursued and by whom

- The process by which a HERS or certification program was developed and implemented or by which a HERS was linked with EEMs

- $\quad$ Future program plans.

In addition, information was gathered on the names of lending institutions currently offering EEMs to borrowers.

The level of activity in the states was much higher than NREL had expected when undertaking this progress report on HERS/EEM activities around the nation. 


\section{Alabama}

No primary information available.

\section{Secondary Information}

\section{U.S. Department of Energy Report (1992)}

Does not have a statewide HERS program.

Two Alabama utilities-Alabama Power Company (Energy-Efficient House-"Good Cents Home") and Alabama Gas Corporation (Energy-Efficient House-"Wise Home")-administer voluntary certification programs (p. 60).

\section{NASEO Survey (1991)}

Alabama state energy officials are considering the Energy Rated Homes of America system. They perceive the lack of resources and building industry opposition as barriers to the development of a HERS program.

\section{Southern Electric International (Good Cents)(1992)}

The following utilities in Alabama have Good Cents programs:

- Alabama Power Company

- Alabama Electric Cooperative

- Baldwin County EMC

- Brundidge Electric

- Central Alabama Electric Cooperative

- City of Andalusia

- City of Elba

- City of Opp

- Clarke-Washington Electric Cooperative

- Coosa Valley Electric Cooperative

- Dixie Electric Cooperative

- Pea River Electric Cooperative

- Pioneer Electric Cooperative

- Southern Pine Electric Cooperative

- South Alabama Electric Cooperative

- Tallapoosa River Electric Cooperative

- Wiregrass Electric Cooperative.

Barakat and Chamberlin, Inc. (1992)

The following utilities have DSM programs:

- Alabama Power Company

- Baldwin County EMC

- Fort Payne Improvement Authority. 


\section{HERS Provider Information Network (Taylor 1993)}

The Alabama Department of Economic and Community Affairs, Division of Science, Technology and Energy, Montgomery, is joining the HERS Provider Information Network.

\section{Vories and George (1991)}

The Alabama Power Good Cents Program, started by the utility in 1977:

- Uses a performance-based rating tool

- Certifies new, single-family and multifamily homes

- Has certified 32,755 single family homes; 31,339 multifamily units. In 1990 , rated $55 \%$ of all new electric homes in service territory

- Conducts some marketing activities; cooperates with builders to advertise program

- Employs cash incentives on heat pumps and electric water heaters (p. 33). 


\section{Alaska}

Source of Information: Barbara Collins, Program Coordinator, Energy Rated Homes of Alaska, 907/563-6773

\section{Background}

In 1984, Municipal Light and Power Company (MLPCO) and Key Bank Alaska (KBA)[a subsidiary of . Key Corporation, based in New York Cityl assembled representatives from the housing industry, homebuilders, real estate associations, and mortgage lenders to discuss the feasibility of linking EEMs with a voluntary, market-driven HERS. Alaska was developing a minimum energy-efficiency standard for new buildings and group members thought that a HERS would provide an incentive for complying. (The ruling was overturned in 1986 due to a technical detail.)

After contacting the Seattle-based Western Resources Institute (WRI)-predecessor of Energy Rated Homes of America (ERHA) - about their rating system, MLPCO approached the Alaska Energy Office (AEO) proposing that the state invest in a rating system. AEO acknowledged the benefits of a voluntary rating system and agreed to finance the purchase of a HERS. Using $\$ 10,000$ of oil overcharge funds, AEO commissioned WRI to develop a home energy rating system tailored to the regional variations in Alaska's climate and energy costs. WRI completed the Alaska HERS in 1986.

Oversight for the HERS program-now referred to as Energy Rated Homes (ERH) of Alaska (AK)-became the responsibility of a housing industry steering committee. AEO developed a rating sheet and hired two contractors-Heat Loss Analysis in Anchorage and Duszynski and Associates in Fairbanks-to conduct the ratings. Under the provisions of the contract signed between AEO and WRI, WRI provided technical support to the Alaska HERS and archived the state's ratings in their database. Initially, ERH of AK offered a one-day seminar to train appraisers to use the HERS rating sheets so that, for an additional fee, they could offer a HERS rating as an auxiliary service. However, in late 1986, Alaska entered a depression and the real estate market collapsed. Only three to five ratings had been performed at that time.

The State did not begin to recover economically until 1989. The Steering Committee, unable to devote the time required to revive the moribund HERS program, requested that AEO assume full administrative responsibility for ERH of AK. Although the state energy office agreed to take over the organization, ERH of AK maintained quasi-independent status, operating as an AEO contractor. AEO did two things to revive the organization:

(1) Hired a part-time staff person to oversee the administration and marketing of the ERH of AK program. Staff marketed the program to the real estate and housing industries, and

(2) Recruited 13 raters throughout the state based on their geographic locations and qualifications. Once AEO selected the raters, they convened in Anchorage for a week-long training session with both classroom and field work. By 1991, 38 raters had been recruited and trained.

ERH of AK also assembled a new advisory committee consisting of both old and new members. The committee served two functions: 
It served as a conduit for disseminating information to the building industry, lending institutions, and real estate interests since members from each of these groups served on the committee.

The advisory committee had two subcommittees: (1) a long-range planning committee, and (2) a curriculum planning committee to develop course materials for real estate agents and appraisers.

\section{Developing a Linkage with EEMs}

At the same time MLPCO approached AEO about HERS in 1984, KBA approached the Alaska Housing Finance Corporation (AHFC) about offering EEMs. In 1986, the AHFC became the first state housing finance agency to adopt an EEM linked to a HERS by adopting the $2 \%$ stretch for homes meeting ERH of AK's Four-Star rating. However, AHFC did not offer EEMs for energy-efficiency improvements to existing homes at time of sale or refinance.

\section{Recent Developments}

Since July 1992, ERH of AK has been administered by the Alaska Housing Finance Corporation (AHFC). In July 1992, the state legislature combined all of the state's housing programs into AHFC. As a result, AHFC is a comprehensive housing agency which administers the state public housing authority, Alaska's Comprehensive Housing Affordability Strategy (CHAS), the HOME program, and Alaska's Low-Income Weatherization and homeless programs. AHFC also serves as a secondary mortgage market for lower and moderate income Alaskan home buyers. In addition, AHFC has a financial partnership with HUD's Indian Housing program, the Supplemental Development Fund.

AHFC and the Alaska HUD office have a number of agreements to share resources and promote affordable, energy-efficient housing. AHFC also has an agreement with the Federal Housing Administration (FHA) to promote energy-efficient housing using ERH of AK.

In addition to the EEM training provided to Alaska's housing industry professionals through FHA, courses on energy-efficient housing have been accredited by the Alaska Board of Certified Appraisers and the Alaska Real Estate Commission. These courses offer appraisers and real estate agents educational credits to meet state relicensing requirements. Alaska's professional home builders are also required to attend a course on energy-efficient home construction in order to obtain a home building license.

ERH of AK is administered as a public/private partnership between AHFC and the housing industry. The rating system is now an integral part of all of the state's affordable housing programs. For example, it is used for state energy code compliance, for EEMs, by housing authorities for the Comprehensive Improvement Assistance Program, and by utilities in their demand-side management programs. ERH of AK is now developing energy rating software that will link the rating system to the Low-Income Weatherization program. The goal is to strengthen both ERH of AK and the Weatherization program by combining resources. It will also provide weatherization clients with the benefits of a HER (e.g., access to EEM's) and build a greater data base on Alaska's housing stock.

ERHA has rated more than 6,000 homes across Alaska since it began. Currently over 2,000 homes are rated annually.

In July 1993, AHFC adopted an Energy-Efficiency Interest Rate Reduction. It provides an interest rate reduction of up to $1 \%$ for homes which are already energy efficient, as determined by an ERH of AK rating of Four Star Plus or greater. It also provides an interest rate reduction of up to $1 \%$ for buyers of 
homes which have been upgraded within the past twelve months $O R$ which are made more efficient within 120 days of purchase. To qualify for this reduction the home's rating must increase by at least one step in the rating program (e.g., Two Star to Two Star Plus).

Alaska is one of the five states chosen to participate in the HUD/FHA energy-efficient mortgage pilot program.

\section{Lending Institutions Known to Offer EEMs}

- Alaska Federal Savings and Loans, Sitka, Juneau, Ketchikan, and Wrangell

- Alaska USA Federal Credit Union, Anchorage

- Alaska Home Mortgage, Anchorage

- Bank of America, Anchorage

- City Mortgage, Anchorage, Eagle River, and Wasilla

- Denali State Bank, Fairbanks

- First Bank of Ketchikan, various branches

- First National Bank of Anchorage, various branches

- First Interstate Bank, Anchorage

- First Union Mortgage Corp., North Carolina, Seattle, and Portland

- Frontier Alaska State Credit Union, Anchorage, Fairbanks, and Kenai

- GMAC Mortgage, various branches

- Key Corporation, Mortgage Inc., branches statewide

- Mount McKinley Bank, Fairbanks

- National Bank of Alaska, branches statewide

- Northland Mortgage, various branches statewide

- Norwest Mortgage, Anchorage, Eagle River, Juneau, Wasilla, and Kenai

- Seattle Mortgage Company, Anchorage.

\section{Secondary Information}

\section{U.S. Department of Energy Report (1992)}

Energy Rated Homes of Alaska (ERH of AK)—a public/private partnership between the State and the housing industry-administers a scaled, performance-based HERS. Although Alaska's HERS program has always been linked with EEMs, the use of EEMs has not been widespread. EEM activities have focused on:

- Educating appraisers, lenders, real estate agents, and builders, and

- Removing institutional barriers that prevent or inhibit the use of EEMs.

The Alaska HERS program has three near-term goals:

- Implementing a program that provides EEM training to appraisers and real estate agents. The course work will qualify as an elective for obtaining a real estate license 
- Finalizing and implementing new HERS agreements with utilities

- Developing and implementing a new Alaska Housing Finance Corporation Energy-Efficient Mortgage (p. 169).

\section{NASEO Survey (1991)}

Alaska has a HERS - based on the Energy Rated Homes of America system - and state officials have had a positive experience with the rating system. Alaska's HERS is linked with EEMs. The officials commented that the lack of national, uniform EEM guidelines was an impediment to linking HERS with EEMs.

Barakat and Chamberlin, Inc. (1992)

The following utilities have DSM programs:

- Alaska Electric Light and Power Company

- Golden Valley Electric Association, Inc.

HERS Provider Information Network (Taylor 1993)

Energy Rated Homes of Alaska, Anchorage, is joining the HERS Provider Information Network.

\section{Vories and George (1991)}

Energy Rated Homes of Alaska, which has operated the state's HERS program since 1986:

- Uses ERHA software (generates a score of 0 to 100 which translates into a Five-Star Rating; Five-Star Rating is equivalent to an Alaska Craftsman Home, sponsored by the Home Builders Association)

- Updated software in 1992; incorporated new minimum state thermal standards

- Rates new and existing single-family homes; is fuel neutral

- Has rated almost 300 homes

- Charges $\$ 50$ per rating; $\$ 35$ subsidy charged to state

- Budgets $\$ 60,000$ to $\$ 70,000$ per year for program costs

- Actively markets program

- Started cash rebate program for those who upgrade rating

- Has link with EEMs; however, not a significant part of ERH of AK's program

- Plans to link HERS program with weatherization program; intends to rate at least 1,000 homes a year as part of weatherization efforts (p. 49). 


\section{PERI (1992)}

Steve Baden: Regional efforts are underway to encourage HERS and EEMs in the Pacific Northwest, including Alaska, Oregon, Washington, and Idaho (10/30/92).

\section{Hughes (1992)}

Under a recent agreement with the State of Alaska, HUD is working with the State's Department of Community and Regional Affairs to promote energy-efficient housing in Alaska. Included in the agreement are the following provisions:

- All HUD-financed new homes must comply with the state's energy-efficiency standard

- HUD recognizes ERH of AK rating for energy-efficient homes, having determined that the rating meets federal energy-efficiency standards

- HUD will allow a debt-to-income stretch of $2 \%$ for homes receiving a Four-Star (or better) rating

- An ERH of AK home energy rating is an alternative to the heat-loss calculations required by HUD regulations

- ERH of AK ratings can substitute for a home energy audit for home buyers wishing to finance energy-efficiency improvements to an existing home in their mortgage loan

- The cost of a home energy rating (up to $\$ 200$ ) can be financed in a mortgage loan

- HUD will incorporate discussion of home energy efficiency and home energy ratings in its underwriter/appraiser workshops.

The Alaska State Home Builders Association collaborated with the state's energy-efficiency industry to lobby for increased state funding for ERH of AK and for the Alaska Craftsman Home Program.

ERH of Alaska has rated 3,300 homes. 


\section{Arizona}

Source of Information: Charlie Gohman, Coordinator of AzHERS, Arizona Department of Commerce, $800 / 352-5499$

\section{Background}

In 1987, the Arizona Department of Commerce Energy Office (EO) launched the Solar Home Program (SHP) to encourage the use of solar energy in new home construction. The SHP linked EEMs with the purchase and installation of solar water heaters. Between 1988 and 1989, 70 to 80 EEMs were processed in conjunction with the SHP.

By 1989, the SHP faced barriers because solar water heaters were not included in the appraisal process. Also, the process was too cumbersome to meet the needs of lenders, real estate professionals, and home builders. In 1989, following attempts to resolve the SHP's difficulties, EO discontinued the program and began developing the Arizona Home Energy Rating System (AzHERS). The new program would:

- Rate a home on its performance, not its energy features

- Estimate heating, cooling, and water heating energy use

- Be based on a 0-to-100 rating scale

- Try to establish a link between a home's value and its energy efficiency

- Be closely linked to HUD's Energy-Efficient Home (EEH) guidelines.

During 1989, EO reviewed several HERS software programs, including REM/Design, Energy Rated Homes of America, and CALRES. Of the three considered, the CALRES system was considered best for AzHERS specifications. Features included the ability to:

- Model evaporative cooling

- Model alternative building materials such as adobe and rammed-earth

- Accommodate Arizona's diverse climate zones

- Model passive solar designs.

In 1990, EO contracted with the Arizona Solar Energy Association (ASEA) to develop and conduct a pilot test of the AzHERS program in Prescott, Arizona. The pilot had two goals: (1) to test the CALRES/Az rating tool, and (2) to establish a link with EEMs.

The pilot was partly successful. CALRES/Az provided reliable estimates of energy use. Ten existing homes and a number of new homes were rated. ASEA verified the accuracy of AzHERS ratings on existing homes by comparing the results with each home's energy bills.

EO and ASEA did not succeed in establishing a linkage between HERS and EEMs for the following reasons:

- Lenders showed little interest in EEMs

- Builders and real estate professionals, although interested, did not actively promote EEMs

- There was very little consumer demand for energy efficiency.

No EEMs resulted from the pilot. In December 1991, EO concluded the Prescott pilot. 
During the Prescott pilot in 1991, EO and HUD's Regional Office began meeting with Arizona utilities about using AzHERS in their builder DSM programs. Both the Phoenix and Tucson regional HUD offices had established AzHERS as the alternative performance compliance method for the EEH prescriptive guidelines. Compliance with existing utility guidelines did not guarantee that a home would qualify under HUD's EEH program. The Arizona Corporation Commission staff recommended that AzHERS be used as the compliance tool in utility DSM programs. By 1992, all of Arizona's regulated utilities were using or had committed to use AzHERS.

\section{Recent Developments}

As the Prescott pilot was drawing to a close in 1991, EO implemented the AzHERS program in Phoenix. Phoenix had a large, new home market and the AzHERS program focused exclusively on rating new homes. Southwest Gas (SWG) and Arizona Public Service Company (AzPSCo) assumed primary roles in promoting the program. SWG and AzPSCo provided the AzHERS ratings, used the results to qualify new construction for EEMs, and provided builders with the paperwork necessary to process EEMs. Of the new homes built in Phoenix in 1992, 50\% (7,000 homes) had been rated by AzHERS. Currently, 30\% of the FHA mortgages for new homes processed by the HUD office in Phoenix use EEMs. Utilities supplying Bullhead City, Colorado Valley, and Sierra Vista are now providing AzHERS ratings on new homes.

In 1991, EO and HUD formed EEM working groups to help develop and implement a procedure for establishing a link between AzHERS and EEMs. These groups have included representatives from the Western Area Power Association, utilities, municipalities, home builders associations, lenders, and real estate professionals. These groups have:

- Implemented the initial inspection process for rated homes

- Developed the AzHERS Point System, a simplified compliance tool for production homes

- Begun developing a program to retrofit existing homes through the HUD 203k Rehabilitation Mortgage.

In 1992 and 1993, these groups have continued to work on the issues listed above. During the past year, the groups have focused on improving the quality of construction of energy-efficient homes and updating the home inspection process. Group members are developing more stringent guidelines and educational material for inspectors, builders, and sub-contractors. The guidelines were completed in July 1993 and will go into effect on January 1, 1994.

In the future, EO plans to:

- Expand AzHERS statewide

- Finalize work on inspection and quality control

- Provide AzHERS ratings on existing homes. EO expects to begin rating existing homes in the Phoenix and Tucson areas by fall 1993. During summer 1993, EO will collaborate with group members to develop a marketing plan 
- Organize a series of workshops for lenders and real estate professionals on EEMs for existing homes. The first workshop, held in December 1992, targeted lenders and real estate agents, and focused on the procedure for underwriting the HUD 203k Rehabilitation Mortgage

- Provide training for auditors for existing homes. Arizona Electric League and Pima College are developing training programs for the Phoenix and Tucson areas. The first session was scheduled for June 1993.

\section{Secondary Information}

\section{U.S. Department of Energy Report (1992)}

In 1992, the Arizona Department of Commerce Energy Office (state energy office) collaborated with HUD, the Western Area Power Administration, the Arizona Corporation Commission, utilities, the building industry, lenders and real estate professionals to develop an Arizona HERS (AzHERS).

With the collaborative's assistance, APS and Southwest Gas (as well as other utilities throughout the state) have incorporated AzHERS into utility-sponsored builder programs. As a consequence, $30 \%$ of new homes in Phoenix and 5\% of new homes in Tucson have received an AzHERS rating. The collaborative is developing a mechanism for expanding AzHERS into the existing home market, which they expected to be in place by the end of 1992 .

Arizona utilities have documented significant projected energy savings resulting from the AzHERS program (p. 147).

\section{NASEO Survey (1991)}

Arizona has a HERS - based on CALRES/Az software-and has had a positive experience with the rating system. The rating system is linked with EEMs.

\section{Southern Electric International (Good Cents)(1992)}

The following utilities in Arizona have Good Cents programs:

- Arizona Public Service Company

- Sulphur Springs Valley Electric Cooperative.

\section{Barakat and Chamberlin, Inc. (1992)}

The following utilities have DSM programs:

- Arizona Public Service Company

- Salt River Project

- Southwest Gas Corporation

- Tucson Electric Power Company. 


\section{HERS Provider Information Network (Taylor 1993)}

The Arizona Department of Commerce Energy Office, Phoenix, has joined the HERS Provider Information Network.

\section{Vories and George (1991)}

1. The Climate Crafted Home Program, operated by the Salt River Project in Phoenix, Arizona, since 1990:

- Uses a point system based on builder guidelines; a designated number of points qualifies a home for certification

- Certifies single-family, electric homes

- Has certified 2,700 homes

- Is not linked with EEMs (p. 73).

2. The Arizona Energy Office has taken the following steps towards implementing a HERS program:

- Adopted a modified version of CALRES software. Eligible structures include new and existing single-family, detached homes

- $\quad$ Finished a pilot program in Prescott, AZ in April 1991

- Began expanding program to include Tucson, $\mathrm{AZ}$

- Began working on a linkage between HERS and EEMs. Arizona rating system accepted by HUD and Fannie Mae. Fifteen EEMs have been processed using the rating system (p. 81).

\section{PERI (1992)}

Deborah Howard: Arizona has instituted a HERS program in conjunction with the utilities. It addresses new homes. It obtained agreement with Fannie Mae and HUD that they will not promulgate mortgages unless the home has obtained a rating. Arizona is discussing with HUD a pilot program to incorporate retrofits and ratings into new mortgages placed on repossessed homes (6/12/92).

Observer: The Arizona HERS program has been integrated into the State Energy Policy (10/30/92).

\section{Other Sources}

Dina Holland, Marketing Department, Southwest Gas, letter to Barbara Farhar: Southwest Gas administers the Energy Advantage Home Program. 


\section{Arkansas}

Source of Information: Morris Jenkins, Deputy Director, Energy Office, Arkansas Industrial Development Commission, 501/682-7377; Ron Hughes, Executive Director, Energy Rated Homes of Arkansas; and Karen Walker, Director of Operations, Energy Rated Homes of Arkansas, 501/374-7827

\section{Recent Developments}

In April 1993, the Governor of Arkansas signed legislation (Act 750 of 1993) directing the State Energy Office (SEO) to initiate an EEM pilot program. In May 1993, HUD selected Arkansas to participate in the national EEM pilot program. The SEO is developing a strategy for implementating the pilot in Arkansas. The SEO plans to approach major banks about the possibility of incorporating EEMs into their loan programs. In March-May 1993, Arkansas' first EEM was processed under DVA's new EEM guidelines.

SEO will meet with utility representatives to explore how EEMs can be linked with DSM programs. The Arkansas Public Service Commission (PSC) ordered Arkansas' six electric utilities to formulate 20-year DSM programs plans. Arkansas Power and Light Company was the first utility to comply with the order and submitted its plan for PSC review in December 1992.

The SEO is streamlining the state's energy-efficiency programs for affordable housing. The office is consolidating Arkansas' housing rehabilitation and weatherization programs (Coordinated Housing Improvement Program or CHIP) and is developing a uniform application to apply for all home assistance programs. Under the restructured procedure, CHIP auditors would rate the homes being considered for assistance, then issue a prioritized list of recommended improvements.

Arkansas currently uses two different software packages for rating homes-Energy Rated Homes (ERH) of Arkansas' program (EZRater) for EEMs and alternative code compliance, and NEAT (National Energy Audit Training) for weatherization program audits. EZRater and NEAT programs perform similar functions; however, EZRater software has the capability to "rate" the energy efficiency of houses, generate Model Energy Code Compliance and archive to the Energy Rated Homes of America national data base. It is accepted for use with EEMs. The SEO or CHIP may request that DOE allow Arkansas to use the ERH rating software in lieu of the NEAT program for home weatherization audits.

CHIP is retaining ERH of Arkansas to train contractors to perform the ratings. In addition, ERH of Arkansas will offer a series of workshops on duct leakage to HVAC licensing boards, health officials, and builders. A special workshop will be offered to the utilities.

The SEO has upgraded the Arkansas Building Code to meet CABO MEC 92 and ASHRAE 90.1 standards and expects the new standards to be implemented by fall 1993.

\section{Lending Institutions Known to Offer EEMs}

- Sears Mortgage Company, Little Rock

- Bank of McCrory, McCrory

- First Financial Mortgage, Little Rock

- Pulaski Mortgage, Little Rock. 


\section{Secondary Information}

\section{U.S. Department of Energy Report (1992)}

No response.

\section{NASEO Survey (1991)}

Arkansas has a HERS and has had a positive experience with the rating system. Arkansas uses the Energy Rated Homes of America system. The rating system is linked with EEMs. Energy officials cited the lack of national, uniform EEM guidelines as a barrier.

Barakat and Chamberlin, Inc. (1992)

The following utilities have DSM programs:

- Arkansas Power and Light Company

- Entex

- Farmers Electric Cooperative Corporation

- Oklahoma Gas and Electric Company.

\section{HERS Provider Information Network (Taylor 1993)}

Energy Rated Homes of Arkansas, Little Rock, is joining the HERS Provider Information Network.

\section{Vories and George (1991)}

Energy Rated Homes of Arkansas, which began operating the state's HERS program in 1988:

- Uses the ERHA system (numerical score of 0-to-100 translated into 0 to Five-Star rating)

- Rates new and existing single-family homes

- Has rated approximately 600 homes

- Charges $\$ 100$ to $\$ 150$ per rating

- Budgets about $\$ 150,000$ per year to run program; does not conduct enough ratings to break even

- Uses no incentives

- Evaluates utility bill data on selected homes to compare actual with predicted energy use

- Conducts limited marketing (p. 51).

\section{Hughes (1992)}

Energy Rated Homes of Arkansas is now training contractors-such as heating and air conditioning contractors, energy specialists, builders, and energy retrofitters-to conduct home energy ratings. ERH of Arkansas provides independent raters with ERH of Arkansas computer software; however, the organization still issues official rating certificates. ERH of Arkansas maintains quality control by conducting periodic spot checks on rated houses and sending questionnaires to consumers whose homes have been rated by independent raters. 
Other Sources

Ron Hughes, Energy Rated Homes of America, to Barbara Farhar: Legislation introduced in the Arkansas General Assembly would require the Arkansas Office of Energy to develop and implement an EEMs pilot program (3/14/93). 


\section{California}

Source of Information: Greg French, Executive Director, California Home Energy Efficiency Rating System (CHEERS), 714/540-0501; David Altscher, Project Manager, Pacific Gas and Electric (PG\&E), 415/973-6077; and Jim Fulkerson, PG\&E, 415/973-5709

\section{Background}

In 1990, representatives from Pacific Gas and Electric Company (PG\&E) approached the California Energy Commission (CEC) with an initiative to establish a uniform statewide system for rating and labeling the energy efficiency of homes and to link the system with EEMs. A linkage between HERS and EEMs would not only help to control the state's energy costs but would also make housing more affordable. CEC supported the idea. PG\&E hired Pacific Management Dynamics (PMD) to establish a non-profit organization (California Home Energy Rating System, Inc. [CHERS]) to oversee the planning, development, and implementation of the initiative.

By October 1990, CEC and PG\&E, working with the contractor, had assembled a Board of Trustees, who voted on the organization's membership, agreed on the principal roles and responsibilities of members, and ratified articles of incorporation and bylaws. Members included investor-owned utilities, municipal utilities, real estate agents, building industry officials, financial institutions, consumer advocates, and the CEC. During its inception, members agreed upon ground rules for decision making, defined the general structure of the organization, and defined the organization's scope and objectives.

CHERS, Inc.'s aim, as defined in its mission statement, is ". . to promote energy efficiency in the residential sector by facilitating the development and implementation of a uniform, market-driven energy rating system for existing and new housing in California." The organization has three goals:

(1) Developing and implementing a HERS for new and existing homes in California

(2) Providing public education and communication, and

(3) Facilitating the means to finance energy efficiency measures.

CHERS, Inc. members agreed to work towards consensus on all decisions. A caveat allowed the group to resort to majority rule if agreement could not be reached; however, the group never used majority rule.

Initially, CHERS, Inc. had a three-tiered administrative structure consisting of a Board of Trustees-to which the organization's administrator reported-and an Advisory Committee, divided into four subcommittees:

(1) Technical Subcommittee-determined the specifications for the HERS software

(2) Media and Communications Subcommittee-handled all public relations as well as communications within the organization

(3) Legal Subcommittee-dealt with legal issues relating to the use of HERS. Lawyers from trusteemember organizations comprised the committee, and

(4) Public Affairs Committee-analyzed and followed pending state legislation pertaining to the HERS. 
During 1991, members of the Technical Subcommittee researched and developed a rating system, completing the technical design of the software in June 1992. The rating system has three elements:

(1) Provides a numeric rating from 1-100 with 100 designating a home using no energy. Homes that meet current California Building Energy Efficiency Standards would receive a rating of 70-75. Only those features permanently built into homes are included in the rating

(2) Provides homeowners with a cost-estimate of gas and electric service for the home (including appliances) based on local utility rates

(3) Provides a list of suggested energy-efficiency improvements so that homeowners can select those improvements which will have the greatest impact on their energy bill.

Ratings are available through private companies and utilities whose employees have been trained and certified to use the tool and who meet CHERS, Inc.'s standards.

\section{Recent Developments}

In November 1992, after completing the technical development of the software and HERS program planning, CHERS, Inc. reorganized, changing its name to California Home Energy-Efficiency Rating System, Inc. (CHEERS, Inc). The new structure reflects a more centralized management style, better suited to the responsibilities of program implementation and administration. CHEERS, Inc. members appointed Greg French executive director to oversee the administration and management of the organization's programs, work with the Steering Committee (which advises on policy, procedures, and goals) and the Advisory Board (members are constituents of groups represented on the Board of Trustees), and oversee the subcommittees. The Executive Director reports to the Board of Trustees.

In May 1992, CHEERS, Inc. members assembled a Mortgage Subcommittee with representatives from the Federal Housing Administration, Freddie Mac, Fannie Mae, a mortgage insurance company, a bank, and a savings and loan company to begin working out the connection with EEMs. During the six months that the committee met, members provided input on the HERS rating label and recommendation sheet and discussed the mechanism for underwriting EEMs. Although participants did not change the ways in which they processed EEMs, they agreed to accept CHEERS, Inc.'s ratings and to use the CHEERS form and recommendation sheet in the underwriting procedure.

CHEERS, Inc. completed a preliminary test of the HERS software in fall 1992, rating over 250 homes during a 45-day period. Statewide implementation of the program began in February 1993 in San Jose (Northern California) where CHEERS, Inc. will conduct about 1200 ratings during a six-month period. The organization extended the program to San Bernardino County in August 1993 and will advance to Sacramento County in October 1993. At first, raters will be trained in Stockton, but as the program extends to other parts of the state, training sites will be decentralized. CHEERS will offer its services statewide in early 1994.

For 1993-94, CHEERS, Inc. will focus on raising customer awareness to increase demand for HERS/EEMs. The organization plans to disseminate information to the public, real estate agents, and developers. During January through March 1993, CHEERS, Inc. met with San Jose banks and mortgage lenders to introduce them to HERS and EEMs, secure a list of lenders willing to offer EEMs, and solicit their ideas on how best to market the HERS/EEM linkage. CHEERS, Inc. will continue its discussions with lenders and local representatives of federal agencies during the statewide roll-out of the program. Each utility has developed its own strategy to market the program to customers. HUD selected California to participate in the FHA EEM Pilot Program. 
In 1992, California enacted legislation requiring the State Energy Resources Conservation and Development Commission to adopt a statewide energy rating program for residential dwellings by January 1, 1994.

\section{Lending Institutions Known to Offer EEMs}

- Western Financial Savings Bank

- General Motors Acceptance Corporation (for new construction).

\section{Secondary Information}

\section{U.S. Department of Energy Report (1992)}

CHERS, Inc., a non-profit organization, developed and is implementing a HERS for the state of California.

The California Energy Commission (state energy office) conducted an EEMs demonstration program in the Turlock Irrigation District's service territory (p.151).

\section{Southern Electric International (Good Cents)(1992)}

The following California utilities have Good Cents programs:

- Los Angeles Department of Water and Power

- Surprise Valley Electric Corporation.

\section{Bonneville Power Administration (Super Good Cents)(1993)}

Surprise Valley Electric Corporation in Alturas, California has a Super Good Cents program.

Barakat and Chamberlin, Inc. (1992)

The following utilities have DSM programs:

- Glendale Public Service Department

- Los Angeles Department of Water and Power

- Modesto Irrigation District

- Pacific Gas and Electric Company

- Palo Alto Electric Utility

- Riverside Public Utilities

- Roseville Electric Department

- Sacramento Municipal Utility District

- San Diego Gas and Electric Company

- Southern California Edison Company

- Southern California Gas Company

- Southwest Gas Corporation

- Turlock Irrigation District.

\section{HERS Provider Information Network (Taylor 1993)}

The following California organizations are joining the HERS Provider Information Network Program: 
- Bay Area Energy Consultants, Palo Alto

- Pacific Gas and Electric, San Francisco

- California Home Energy Efficiency Rating System (CHEERS), Costa Mesa

- California Energy Extension Service, Sacramento.

\section{Vories and George (1991)}

1. The Power Saver Homes program started by the California Modesto Irrigation District in 1986:

- Certifies homes meeting a prescriptive list of standards

- Certifies new, single-family homes; emphasizes production, tract houses

- Has certified over 700 homes

- Is not linked with EEMs

- Offers $\$ 400$ to $\$ 600$ rebates to builders for installing energy-efficient air conditioners

- Plans to upgrade list of prescriptive features as well as begin certifying multifamily buildings (p. 69).

2. Noted the development of the CHERS program at cost of $\$ 500,000$ in 1991 (p. 82).

\section{PERI (1992)}

Jim Fulkerson: CHERS is in the process of finalizing the development of the tool they will use for conducting ratings. They are planning pilot training activities in San Jose and the southern part of the state. They have been discussing linkage with EEMs, cooperative marketing efforts, and other processrelated issues with lenders and local representatives of federal agencies $(6 / 12 / 92)$.

Greg French: CHERS is currently testing its software. They will be rating over 300 homes during the next 45 days as a test of the program. They expect to start their first pilot program at a northern California location in January and the second at a southern location in April (10/30/92).

FmHA (1993)

California building codes/standards meet or exceed CABO MEC $89 .^{1}$

${ }^{1}$ EPACT directs HUD and FmHA to develop energy-efficiency standards that meet or exceed CABO MEC 1992 for new public and assisted housing and single- and multifamily homes. FmHA information on qualifying states is based on information contained in Better Building Codes for Energy Efficiency, published by the Alliance to Save Energy, and in the 1991 edition of Energy Directory, published by the National Conference of States on Building Codes and Standards. 


\section{Colorado}

Source of Information: Jay Luboff, Consultant, Colorado Office of Energy Conservation, 303/541-9325

\section{Recent Developments}

Colorado is developing an Energy Rated Mortgage Program (CERMP) for single-family residential housing, both new and existing. On February 1, 1993, the Colorado Office of Energy Conservation (OEC) hired Jay Luboff, former Director of Western Sun-Washington and founder of Energy Rated Homes of America, to staff the launching of this statewide initiative. The OEC is asking the CEO of the Public Service Company of Colorado (PSCo) to co-chair a Policy Oversight Committee on energy rated mortgages. The other co-chair will be a leading banker in the state.

The Policy Oversight Committee will have representatives from "all of the interests," including housing interests (builders, appraisers, etc.), the Colorado Housing Finance Agency (CHFA), the city of Denver, the Colorado Division of Housing, and Colorado REAs, as well as PSCo and the banking community. The committee's purpose will be to provide guidance to staff, thereby providing legitimacy to the effort. Mr. Luboff hopes the committee can be convened by the end of April 1993. Steps in the process include reviewing the work to date. The work to date is a comprehensive plan for implementing the CERMP. With committee approval, the implementation effort will go forward, involving the establishment of a coalition structure for implementing a HERS linked with EEMs in Colorado. The structure will likely be called the Colorado Energy Mortgage Authority (CEMA). It is yet to be decided where this entity would be housed. Some of the options are (1) independently as a 501(c), (2) within the OEC, and (3) within the CHFA. The plan is to field the program by late summer 1993.

The city of Denver is a partner in the program development and is submitting a grant proposal to the Urban Consortium Task Force to assist the effort by analyzing where program focus can be most beneficial in the Denver area and by taking a lead in community relations amongst the various participants (builders, lenders, energy service companies, etc.) who will benefit from the program. Steve Andrews, former Denver metro Home Builders Association energy committee chair, and Steve Fonte, director of the Denver Office of Environmental Affairs, are working on this effort. Colorado hopes to be one of the HUD EEM pilot states, but if this does not happen the state will move forward using existing VA, HUD and CHAFA programs to stimulate the CERMP.

As an additional incentive to consumers to use EEMs, program staff would like to see home buyers offered a $.0025 \%$ interest break (i.e., lower than prevailing rates) on very energy-efficient homes, offered at the national level by FNMA, FHLMC, HUD and VA. The OEC may provide funding for other incentives using some of its petroleum violation escrow (PVE) funds. In addition, PSCo's DSM programs could provide incentives; these would need to be determined through the integrated resource planning (IRP) process of the Colorado Public Utilities Commission (PUC). For example, PSCo (or the state) could buy-down the cost of improvements, contribute the incremental costs of down payment, pay for the HERS and other incentives, or provide arranging services.

The Colorado CERMP is planning to use a technical tool that blends REM/Rate and ERHA tools with output identical to that of ERHA. This would be the same tool that PSCo uses to certify homes under its "Ideal Energy Home" program. The CERMP is looking at the costs and benefits of using a blowerdoor test as part of the rating service. The plan, as seen now, is for PSCo to enter into an agreement with the CEMA to do the rating and certification (similar to the RISE utility agreement in Rhode Island) using home inspectors/raters certified by the state. 
PSCo's Ideal Energy Home program, using a certification approach, has been active in involving local banks and taking advantage of the $2 \%$ ratio stretch offered by Fannie Mae for new electrically heated housing.

The Mortgage Bankers Association (MBA) designated Bruce Bowler of Universal Lending Corporation, Denver, CO, to represent MBA on the HERS Council Board of Directors.

\section{Secondary Information}

\section{U.S. Department of Energy Report (1992)}

Does not have a statewide HERS program (p. 127).

\section{NASEO Survey (1991)}

Colorado has a HERS - based on the Fort Collins REM/Rate system -in a limited number of communities. The rating system does not tie into EEMs.

Barakat and Chamberlin, Inc. (1992)

The following utilities have DSM programs:

- Lamar Utilities Board

- Public Service Company of Colorado.

\section{HERS Provider Information Network (Taylor 1993)}

The Colorado Office of Energy Conservation, Denver, is joining the HERS Provider Information Network.

Vories and George (1991)

1. The Colorado Office of Energy Conservation, which expects to implement a HERS pilot program in 1992 :

- Is developing HERS software (0-to-100 rating scale)

- Plans to rate new and existing single- and multifamily residences; will maintain fuel neutrality

- Plans to charge a fee for ratings

- Is using approximately $\$ 960,000$ in oil overcharge funds to develop and market a HERS

- Hopes to establish a linkage between HERS and EEMs (p. 84).

2. Fort Collins Power and Light-through the ENERGY SCORE Program launched in 1989conducts home energy ratings in the City of Fort Collins:

- Has rated over 350 homes (both new or existing single- or multifamily units) 
- Ratings are accepted by HUD, Fannie Mae, and Freddie Mac for the purchase of new homes; no effort has yet been made to establish a HERS/EEM linkage for existing homes (p. 65).

\section{Hughes (1992)}

The Fort Collins Light and Power ENERGY SCORE program:

- Uses the original REM/Rate software (0-to-100 scale; fuel type of home is designated)

- Rated 500 new and existing homes in the past $3 \frac{1}{2}$ years

- Is linked with EEMs offered by nine local lenders. HUD and Fannie Mae accept ENERGY SCORE ratings. Adaptation of ratings to Freddie Mac guidelines is under discussion. City of Fort Collins offers a $0 \%$ interest loan program for loans up to $\$ 2,000$. The city uses ENERGY SCORE ratings to determine applicant eligibility for these loans

- Offers workshops for area builders, home owners, and home buyers

- Participates in the HERS Council. 


\section{Connecticut}

Source of Information: Alan Johanson, Assistant Director, Policy and Planning Division, Office of Policy and Management, 203/566-2800; Virginia Judson, Lead Policy Analyst, Policy and Planning Division, Office of Policy and Management, 203/566-3394; and John Ruckes, Lead Policy Analyst. Policy and Planning Division, Office of Policy and Management, 203/566-5898

\section{Background}

In the late 1980s, state energy officials attempted to implement a linkage between HERS and EEMs. Lack of interest in the initiative eventually led to its discontinuation.

In 1990, Connecticut passed legislation (CT Public Act 90-304) requiring that a HERS be developed and implemented. The legislation established no timeframe for implementing a HERS program.

\section{Recent Developments}

During the past three years, state energy officials have collected information on HERS programs in several states (Energy Homes of America [ERHA], ERH of Vermont, and ERH of Virginia). Policy and Planning Division (PPD) staff are interested in developing a HERS program, but lack of funds and staff are barriers to fulfilling the legislative mandate.

OPM staff are currently involved with utility collaborative teams directed by the Public Utility Commission (PUC). The teams-one for each of Connecticut's five major gas and electric utilities-include representatives from OPM, the PUC, a public interest group, and a utility. Team members discuss the utility's budget for the upcoming year and develop the utility's residential, industrial, and commercial DSM programs.

\section{Secondary Information}

\section{U.S. Department of Energy Report (1992)}

No programs in place (p. 6).

NASEO Survey (1991)

Connecticut state energy office is developing a HERS. SEO officials find the lack of resources a barrier to HERS development.

\section{Southern Electric International (Good Cents)(1992)}

United Illuminating in Connecticut has a Good Cents program.

\section{Barakat and Chamberlin, Inc. (1992)}

The following utilities have DSM programs:

- Connecticut Natural Gas Corporation

- Northeast Utilities

- Southern Connecticut Gas Company

- United Illuminating Company. 
HERS Provider Information Network (Taylor 1993)

The Connecticut Office of Policy and Management, Hartford, is joining the HERS Provider Information Network.

\section{Vories and George (1991)}

The Connecticut Office of Policy and Management, which is developing a HERS:

- Is required by Connecticut legislation to integrate a HERS into state residential energy programs

- Expects to complete preliminary research on the rating system between January and March 1992

- Anticipates primary focus of rating system to be new construction

- Does not know if program will be linked with EEMs (p. 86).

FmHA (1993)

Connecticut building codes/standards meet or exceed those of CABO MEC 89. 


\section{Delaware}

No primary information available.

\section{Secondary Information}

\section{U.S. Department of Energy Report (1992)}

Delmarva Power is Delaware's largest utility and is the state's only regulated utility. Delmarva Power provides customers with several services related to home and building energy efficiency, including:

- Super E+ Program: provides an energy-efficiency standard accepted by homebuilders throughout Delmarva's service territory

- Home energy audit: recommends ways to increase the energy efficiency of customer homes (p. 42).

NASEO Survey (1991)

Delaware is interested in developing a HERS. Lack of resources was cited as a barrier.

Barakat and Chamberlin, Inc. (1992)

Delmarva Power and Light Company runs a DSM program.

\section{HERS Provider Information Network (Taylor 1993)}

The Division of Facilities Management, Dover, is joining the HERS Provider Information Network.

Vories and George (1991)

The Super E+ Home certification program, operated by Delmarva Power and Light since 1982:

- Provides prescriptive energy-efficiency guidelines for builders

- Certifies single- and multifamily dwellings

- Has certified over 8,000 homes (10\% to $20 \%$ of new homes)

- Is establishing a link with EEMs; has been accepted by the secondary mortgage market (p. 61). 


\section{District of Columbia}

No primary information available.

Secondary Information

U.S. Department of Energy Report (1992)

Does not have a statewide HERS program (p. 45).

Barakat and Chamberlin, Inc. (1992)

The following utilities have DSM programs:

- Potomac Electric Power Company

- Washington Gas Light Company.

HERS Provider Information Network (Taylor 1993)

The Alliance to Save Energy is joining the HERS Provider Information Network. 


\section{Florida}

Source of Information: Alice Lewis, Energy Analyst, Department of Community Affairs, Energy Code Program, 904/487-1824 and Daryl O'Connor, Community Assistance Consultant, Division of Housing and Community Development, Department of Community Affairs, 904/488-2475

\section{Recent Developments}

In April 1993, Florida passed legislation (CS/HB 1045) directing the Department of Community Affairs (DCA) to develop and maintain a statewide, uniform system for rating the energy efficiency of buildings. The rating system will apply to all new and existing public, commercial, and residential buildings in the state and will be phased in over a three-year period beginning January 1, 1994.

The Florida Building Energy-Efficiency Act directs the DCA to establish a voluntary working group of individuals interested in the HERS or energy efficiency (such as mechanical and electrical engineers, architects, public utilities, and builders) to advise the DCA in developing a rating system. This working group will be responsible for coordinating educational programs for designers, builders, businesses, and others interested in facilitating the incorporation of the HERS into existing practices. In addition, the DCA is responsible for developing a training and certification program to certify raters.

The act requires that a building's energy-efficiency rating be disclosed in writing to a potential purchaser on or before closing day. If no rating has been established, a potential purchaser must be notified that he or she has the option of having an energy-efficiency rating on the building performed. The prospective purchaser will receive a brochure prepared by the DCA containing the following information:

- How to analyze the building's energy-efficiency rating

- Comparisons with statewide averages for new and existing construction of that class

- Methods to improve the building's energy-efficiency rating, and

- A notice to residential purchasers that the energy-efficiency rating may qualify the purchaser for an energy-efficiency mortgage from lending institutions.

In addition, property owners wishing to install solar collectors or other energy devices based on renewable resources will be able to do so.

Energy officials at the Department of Community Affairs (DCA) are developing a Florida HERS based on the Florida State Energy Code. The code is updated every two years and exceeds CABO MEC 91 standards. Fannie Mae has endorsed the Florida code, thus qualifying all newly built homes for mortgages with a $2 \%$ ratio stretch.

Florida state energy officials are monitoring HERS Council activities. The DCA plans to implement a HERS (for new residential homes) linked with EEMs by January 1994. 


\section{Secondary Information}

\section{U.S. Department of Energy Report (1992)}

Does not have a statewide HERS program (p. 62).

\section{NASEO Survey (1991)}

Florida has a HERS based on the state's building code. The rating system is not linked with EEMs. State energy officials commented that lack of resources to properly promote and manage the program, as well as the state's special climate needs, are barriers to the development of a Florida HERS.

\section{Southern Electric International (Good Cents)(1992)}

The following Florida utilities have Good Cents programs:

- Choctawhatchee Electric Cooperative

- Escambia River Electric Cooperative

- Gulf Coast Electric Cooperative

- Gulf Power Company

- Lee County Cooperative.

Barakat and Chamberlin, Inc. (1992)

The following utilities have DSM programs:

- Florida Power and Light Company

- Florida Power Corporation

- Gainesville Regional Utilities

- Gulf Power Company

- Jacksonville Electric Authority

- Lakeland Department Electric and Water Utilities

- Leesburg Electric Department

- Orlando Utilities Commission

- Tampa Electric Company.

\section{HERS Provider Information Network (Taylor 1993)}

The Florida Energy Office, Tallahassee, is joining the HERS Provider Information Network.

\section{Vories and George (1991)}

1. The Gulf Power Company Good Cents program, launched in 1976:

- Provides a prescriptive list of requirements for thermal envelope, mechanical heating and cooling equipment, and domestic hot water systems (pass/fail program)

- Has certified approximately 100,000 homes

- Certifies new single-family and multifamily homes; gas and electric (although the latter is emphasized) 
- Has an annual budget of $\$ 850,000$

- $\quad$ Promoted linkage with EEMs; no information on the outcome of that effort (p. 37).

2. The Florida Building Energy Code program, developed by the Florida Department of Community Affairs in 1979:

- Is revised every two years (state-mandated)

- Uses Energy Performance Level (on a scale of 0 to 100 - " 0 " is most efficient) to determine if a building complies with the state energy code; hopes this rating scale can be used as a HERS

- Is a mandatory program

- $\quad$ Rates all types of buildings

- Is not directly linked with EEMs (p. 87).

\section{PERI (1992)}

Steve Baden: Florida is developing legislation to create a HERS (10/30/92).

\section{FmHA (1993)}

Florida building codes/standards meet or exceed those of CABO MEC 89. 


\section{Georgia}

Source of Information: Phil Whitlow, Director of Conservation, Office of Energy Resources, 404/6565176

\section{Recent Developments}

The state has no plans to develop a HERS program. The state is currently focusing on implementing a revised State Energy Code for Buildings, which will go into effect in January 1994. Two Georgia utilities-Georgia Power Company (Good Cents) and Atlanta Gas Light Company (Energy Wise Home)-have their own rating systems.

The state will continue to monitor the success of HERS in other areas, and if the demand from consumers, real estate professionals, builders, and others increases, serious consideration will be given toward initiating a system for Georgia.

\section{Lending Institutions Known to Offer EEMs}

General Motors Acceptance Corporation (GMAC).

\section{Secondary Information}

\section{U.S. Department of Energy Report (1992)}

Does not have a statewide HERS program (p. 63).

\section{NASEO Survey (1991)}

Georgia is interested in developing a HERS. Lack of resources and information were cited as barriers.

\section{Southern Electric International (Good Cents)(1992)}

The following Georgia utilities have Good Cents programs:

- Altamaha Electric Membership Cooperative

- Amicalola Electric Membership Cooperative

- Canoochee Electric Membership Cooperative

- Carroll Electric Membership Cooperative

- Central Georgia Electric Membership Cooperative

- City of Griffin

- Coastal Electric Membership Cooperative

- Cobb Electric Membership Cooperative

- Colquitt Electric Membership Cooperative

- Cowetta-Fayette Electric Membership Cooperative

- Excelsior Electric Membership Cooperative

- Flint Electric Membership Cooperative

- Georgia Power Company

- Grady County Electric Membership Cooperative

- GreyStone Power Corporation

- Hart County Electric Membership Cooperative 
- Irwin County Electric Membership Cooperative

- Jackson Electric Membership Cooperative

- Lamar Electric Membership Cooperative

- Little Ocmulgee Electric Membership Cooperative

- Middle Georgia Electric Membership Cooperative

- Mitchell Electric Membership Cooperative

- Ocmulgee Electric Membership Cooperative

- Oconee Electric Membership Cooperative

- Oglethorpe Power Corporation

- Okefenoke Rural Electric Membership Cooperative

- Pataula Electric Membership Cooperative

- Planters Electric Membership Cooperative

- Rayle Electric Membership Cooperative

- Satilla REMC

- Savannah Electric Power Company

- Sawnee Electric Membership Cooperative

- Slash Pine Electric Membership Cooperative

- Snapping Shoals Electric Membership Cooperative

- Southern Electric International

- Sumter Electric Membership Cooperative

- Tri-County Electric Membership Cooperative

- Troup Electric Membership Cooperative

- Upson County Electric Membership Cooperative

- Walton Electric Membership Cooperative

- Washington Electric Membership Cooperative.

Barakat and Chamberlin, Inc. (1992)

The following utilities have DSM programs:

- Adel Light Department

- Albany Water, Gas and Light Commission

- Barnesville Electric Department

- Camilla Electric Department

- Carroll Electric Membership Cooperative

- Cobb Electric Membership Cooperative

- Flint Electric Membership Cooperative

- Georgia Power Company

- Griffin Electric Department

- Jackson Electric Membership Cooperative

- Oglethorpe Power Corporation

- Planters Electric Membership Cooperative

- Thomasville Water and Light Department

- Troup County Electric Membership Cooperative.

HERS Provider Information Network (Taylor 1993)

The following Georgia organizations are joining the HERS Provider Information Network:

- Southern Electric International Good Cents Division, Atlanta

- Georgia Office of Energy Resources, Atlanta. 


\section{Vories and George (1991)}

The Georgia Power Good Cents program, offered since 1977:

- Provides a prescriptive list of standards that builders must meet

- Certifies new, electric single- and multifamily homes

- Has certified over 100,000 homes (40\% of annual new electric home market)

- Is linked with EEMs only through General Motors Acceptance Corporation (GMAC) (p. 35).

FMHA (1993)

Georgia building codes/standards meet or exceed those of CABO MEC 89. 


\section{Hawaii}

Source of Information: Carolyn Shon, Energy Conservation Program Manager, Energy Division, 808/587-3810, and Elizabeth Ramen, Energy Conservation Analyst, Energy Division, Department of Business, Economic Development and Tourism, 808/587-3806

\section{Recent Developments}

The Department of Business, Economic Development and Tourism has responsibility for developing a HERS in Hawaii by 1996. In Hawaii, residential energy-efficiency requirements differ from those on the mainland, where HERS programs emphasize heating efficiency and home weatherization. In Hawaii, water heating comprises approximately $40 \%$ of the household utility bills. Hawaii plans to develop a HERS based on its model energy code. The code meets ASHRAE 90.1 standards and is presently under review by Hawaii's four counties - Oahu, Maui, Kauai, and Hawaii (Big Island). County adoption of the code is voluntary.

In July 1993, Energy Division (ED) officials plan to meet with representatives from two major banks-the Bank of Hawaii and the First Hawaiian Bank-to promote EEMs. To garner lender support for EEMs, energy officials will present information on state support for and the cost effectiveness of energy-efficiency measures. The meeting will be used to:

- Convey the results of a 1992 report on cost-benefit analysis of heat pumps and solar water heaters by Dr. Peter Flachsburt of the University of Hawaii entitled, "Financial Impacts on Home Buyers of Mandated Energy Efficiency Improvement in Water Heaters for State Affordable Housing Projects." Flachsburt found that heat pumps and solar water heaters were cost effective in Hawaii

- Review a 1990 HUD decision to allow a $1 \%$ increase in the ratio of the mortgage payment whenever an FHA-approved solar domestic hot water heater system is used in new construction

- Explain state tax credits for solar systems (35\% credit) and heat pumps ( $20 \%$ credit).

ED will schedule similar meetings with real estate professionals and professional associations. In addition, the division plans to develop and disseminate a brochure on EEMs.

The Governor signed a bill passed by the state legislature in 1992 requiring the State Housing Finance and Development Corporation (HFDC) to install a percentage of solar water heating equipment at any residential development project built with state funds, located on state land, or otherwise subsidized by the state.

\section{Secondary Information}

\section{U.S. Department of Energy Report (1992)}

No programs or legislation (p. 163).

NASEO Survey (1991)

Hawaii is developing a HERS. 
Barakat and Chamberlin, Inc. (1992)

Hawaiian Electric Company, Inc. runs a DSM program.

\section{Other Sources}

Letter from Maurice H. Kaya, Energy Program Administrator, Hawaii Department of Business, Economic Development and Tourism, to DOE: Hawaii is working to increase home energy efficiency:

- In 1990, HUD revised its criteria for qualifying energy-efficient homes in Hawaii to accommodate climatic differences. HUD agreed to allow a $1 \%$ stretch in the debt-to-income ratio whenever an FHA-approved solar domestic hot water system is used in new construction

- In 1992, Hawaii passed legislation requiring the State Housing Finance and Development Corporation (HFDC) to:

- Install solar water heating equipment at any residential development project built with state funds, located on state land, or otherwise subsidized by the state

- Require installation of solar water heating equipment at a level of $30 \%$ in 1993, $40 \%$ in 1994, and 50 percent in 1995.

The Governor was still considering the bill at the time Kaya wrote the letter (5/14/92).

Letter from Elizabeth Ramen, Energy Conservation Analyst, Hawaii Department of Business, Economic Development and Tourism, to Barbara Farhar (NREL): Five Hawaii utilities run DSM programs:

- Hawaii Electric Company (also listed by Barakat and Chamberlin 1992)

- Hawaii Electric Light Company

- Maui Electric Company

- Kauai Electric

- Gasco (7/16/93). 


\section{Idaho}

No primary information available.

\section{Lending Institutions Known to Offer EEMs}

- Farmers and Merchants State Bank, Boise

- $\quad$ First Security Bank of Idaho, Boise

- Westone Bank, Boise

- Key Bank of Idaho, Boise

- Washington Federal Savings, Boise

- $\quad$ Security Pacific Bank of Idaho.

\section{Secondary Information}

\section{U.S. Department of Energy Report (1992)}

As part of the Bonneville Power Administration's (BPA) regional effort to promote EEMs, the Idaho State Energy Office (SEO) is encouraging the use of EEMs by providing technical assistance to home buyers and lenders. In addition, the SEO assists in processing EEMs for manufactured homes. Idaho publishes a statewide newsletter on EEMs.

The Idaho SEO's near-term goals include:

- Persuading appraisers and lenders to allow credit for energy-efficiency improvements and lower energy costs in Model Conservation Standard (MCS) homes

- Integrating the EEM mechanism into existing finance methodology

- Providing information on energy-efficient homes to utilities, jurisdictions, home buyers, builders, and others

- Assisting in arranging home loans which allow EEM benefits (p. 172).

NASEO Survey (1991)

State energy officials are interested in developing a HERS. An official commented that lack of resources impeded the development of a HERS.

\section{Southern Electric International (Good Cents)(1992)}

The following Idaho utilities have Good Cents programs:

- Bonneville Power Administration-Boise District

- Bonneville Power Administration-Idaho Falls District

- City of Heyburn

- City of Idaho Falls

- Clearwater Power Company

- East End Mutual

- Fall River Electric Cooperative

- Idaho County Light and Power 
- Idaho Power Company

- Kootenai Electric Cooperative, Inc.

- Lost River Electric Cooperative

- Northern Lights, Inc.

- Raft River Electric Cooperative

- Riverside Electric Cooperative

- Rural Electric Company

- Salmon River Electric Cooperative

- South Side Electric

- Unity Light and Power Company

- Utah Power and Light.

Bonneville Power Administration (Super Good Cents)(1993)

The following Idaho utilities have Super Good Cents programs:

- Clearwater Power Company

- East End Mutual

- Fall River Electric Cooperative

- City of Heyburn

- Idaho County Light and Power Cooperative

- City of Idaho Falls

- Kootenai Electric Cooperative

- Lost River Electric Cooperative

- Northern Lights, Inc.

- Raft River Electric Cooperative

- Riverside Electric Cooperative

- Rural Electric Company

- Salmon River Electric Cooperative

- City of Soda Springs

- South Side Electric

- Unity Light and Power Company.

Barakat and Chamberlin, Inc. (1992)

The following utilities have DSM programs:

- Bonneville Power Administration (BPA)

- Idaho Power Company.

PERI (1992)

Steve Baden: Idaho is part of regional efforts to encourage HERS and EEMs in the Pacific Northwest, along with Alaska, Oregon, and Washington (10/30/92).

FmHA (1993)

Idaho building codes/standards meet or exceed those of CABO MEC 89. 


\section{Other Sources}

Javier Figueroa, Program Manager, Lender/EEMs, Washington State Energy Office, letter (and enclosures) to Barbara Farhar: In 1991-1992, a collaborative was formed involving all manufacturers of factory-built homes, utilities, and state energy offices in Oregon, Idaho, Washington, and Montana to design and implement a program to improve the energy efficiency of all electrically heated manufactured homes built in the four-state region. "The Northwest Power Planning Council (NWPPC) first proposed such a project in their 1991 Northwest Conservation and Electric Power Plan.

The Manufactured Homes Acquisition Program (MHAP) works in the following way:

- Bonneville Power Administration (BPA) reimburses home manufacturers $\$ 2,500$ for the added cost of installing energy-efficiency features to the houses they construct

- Utilities will then pay BPA all or a portion of the money advanced to the manufacturer (plus $\$ 150$ to cover administrative costs) for every one of these homes sited in the utility's service territory

- $\quad$ BPA is collecting data from states and utilities on the location of MHAP houses; BPA bills a utility for each MHAP house located in the utility's service territory.

BPA has agreed to serve for four years as the administrative agent for the MHAP program. MHAP homes are eligible for HUD-insured mortgages (4/24/92).

James Nybo, Conservation Analyst, Northwest Power Planning Council, letter (and enclosure) to Barbara Farhar: In late 1991, the BPA, in collaboration with the NWPPC and the state energy offices in the Northwest, started implementing a Regional Energy-Efficient Mortgage Strategy (REEMS).

The purpose of the effort is to make EEMs for site-built and manufactured housing more readily available. Documenting EEMs' impact on buyer purchasing power and supporting ongoing review of state codes are additional aspects of the REEMs program.

The NW collaborative is working to achieve the following goals by June 1993:

- Secure agreement from HUD to support manufactured housing loans through the EEM program

- Secure support from Fannie Mae, Freddie Mac, and PMI (a mortgage insurer)

- Develop a labeling system for all new and previously certified MCS homes

- Develop a tracking system to (a) document the number of EEMs processed and (b) analyze their effect on purchasing power and housing affordability

- Develop and implement marketing strategy

- Provide education, training, and technical assistance to the home finance, sales, and construction industries; set up licensing and continuing education courses for real estate professionals, appraisers, and lending industries

Nearly $30 \%$ of all electrically heated homes in the Northwest are constructed in factories. Approximately 10,000 to 13,000 such homes are manufactured each year in the region. 
- Announce and implement terms of agreement with HUD, Fannie Mae, Freddie Mac, and PMI

- Implement labeling program and tracking system (5/6/92). 


\section{Illinois}

Source of Information: John Marley, Mechanical Engineer, Illinois Department of Energy and Natural Resources, 217/785-2007; and Rose Geier-Wilson, Visiting Housing Research Specialist, Small Homes Council/Building Research Council, University of Ilinois at Champaign, 217/333-1801

\section{Recent Developments}

In 1989, energy officials at the Illinois Department of Energy and Natural Resources (DENR), recognized the need for a voluntary home energy audit package. DENR had been unable to implement a state energy code and, concomitantly, the federal legislation mandating the Residential Conservation Service (RCS) program expired. State energy officials began to consider HERS programs as an alternative to RCS audits.

In 1990, the Illinois Department of Energy and Natural Resources (DENR) contracted with the Small Homes Council (SHC) at the University of Illinois at Champaign to develop a HERS program called EnergyWise Homes of Illinois. In the initial phases of its planning, SHC put together a Technical Advisory Committee comprised of representatives of utilities, builders, analysts, real estate agencies, and lending institutions. The Advisory Committee resolved issues (such as fuel neutrality) and reviewed findings on rating systems.

SHC researched existing HERS programs, selecting two HERS software packages-REM/Rate and REALI. (Residential Energy Analysis for Loads in Illinois) - to test on 30 homes in the Champaign area. SHC presented the results to the Advisory Committee for evaluation. The differences in format and outputs of both REM/Rate and REALI provided DENR with guidelines for developing a software package to meet Illinois' needs. DENR officials elected to use REM/Rate software which provides fuel-neutral ratings and is based on a set standard of energy efficiency.

In September 1991, DENR awarded SHC a grant to administer a HERS/EEM pilot project in central Illinois encompassing Champaign, Urbana, Decatur, Bloomington, Springfield, and several small towns and unincorporated areas. The program is supported with PVE funds. DENR officials chose to work with Illinois University at Champaign instead of establishing a non-profit corporation; the network of state universities provided a potential medium for extending HERS to other regions in the state. The HERS program became an associate member of ERHA because staff members felt that vital information and procedures were being reinvented every time a new HERS program came on line. They believed that it would be more effective to join with an established organization, at a time when the HERS Council did not yet exist.

The pilot program has three overlapping phases:

Phase 1: In 1990, conduct approximately 30 free ratings to test the accuracy of REM/Rate in central Illinois (Champaign area) and have results evaluated by the Technical Advisory Committee

Phase 2: $\quad$ Train private raters to use REM/Rate and continue to offer free ratings in the central Illinois area. Since 1990, approximately 125 homes have been rated. Although this phase was to conclude in late 1992, a Citizen Action Group in Lake County, Mlinois (north of Chicago) contacted John Marley at DENR to arrange for home energy ratings in their area. By mid-April 1993, DENR and SHC had rated approximately 25 to 30 Lake County homes. SHC is marketing the HERS program through low-budget means such as radio and television interviews, press releases, and radio announcements 
Phase 3: $\quad$ Link HERS with EEMs. During Phase II, DENR and SHC developed a strategy to forge a link between HERS and EEMs. In March 1992, DENR sponsored a lender training seminar, led by Jim Curtis, to introduce central Illinois lenders to the concept of EEMs. Approximately 20 lenders out of 120 that were contacted attended. Only one lender out of the total number invited had prior knowledge of EEMs. Although lenders who attended the seminar expressed interest in EEMs, they were deterred by the lack of uniform EEM guidelines and found the paperwork confusing. Lenders did agree to offer EEMs if consumers requested them; however, they were unwilling to market EEMs.

In spring 1993, DENR and SHC continue to pursue a link between HERS and EEMs by contacting lenders in the pilot program areas. Lenders in Lake County have expressed interest in EEMs, finding the idea of selling a new product attractive. During 1993, DENR and SHC plan to meet with representatives from several Mlinois banks, including Tallman Bank in Chicago (which has been interested in EEMs and housing affordability), a central Illinois bank, and Champion Savings and Loan. DENR also hopes to involve the Illinois Housing and Development Authority, which provides funds for low-income housing.

SHC expects the east central Illinois pilot to conclude in November 1993. The state government currently faces budget uncertainties and funding for the HERS/EEMs program is guaranteed only through November. Although SHC believes that those exposed to the Illinois HERS program are beginning to realize the value of the ratings, it is unclear whether or not homeowners would pay a fee for the HERS as a stand-alone service.

\section{Lending Institutions Known to Offer EEMs}

- Tallman Bank, Chicago

- Busey Bank, Urbana

- Champion Federal Savings and Loan, Bloomington

- Bank One Champaign Urbana, Champaign

- First of America Bank Champaign County, Urbana.

\section{Secondary Information}

\section{U.S. Department of Energy Report (1992)}

Does not have a statewide HERS program (p. 83).

\section{NASEO Survey (1991)}

Illinois is developing a HERS.

\section{Southern Electric International (Good Cents)(1992)}

Commonwealth Edison Company in Illinois has a Good Cents program.

\section{Barakat and Chamberlin, Inc. (1992)}

The following utilities have DSM programs:

- Central Illinois Light Company

- Commonwealth Edison Company

- Illinois Power Company 
- Iowa - Illinois Gas and Electric Company

- Northern Ilinois Gas Company

- $\quad$ Springfield City Water, Light, and Power

- The Peoples Gas Light and Coke Company.

Utility Data Institute (1992)

Central Illinois Public Service Company runs a DSM program.

HERS Provider Information Network (Taylor 1993)

The Home Energy Rating System of Illinois, Champaign, is joining the HERS Provider Information Network.

\section{PERI (1992)}

Steve Baden: There are efforts on the state level to develop collaboration between government, utilities, and industry for development of HERS in nlinois (10/30/92).

\section{Hughes (1992)}

The Illinois Department of Energy and Natural Resources is implementing a HERS program (EnergyWise Homes) in east central Illinois:

- The Architectural Energy Corporation assisted in the training of a dozen raters in April 1992. Fortyfive homes have been rated since then

- Jim Curtis, in cooperation with ERHA, conducted lender training in March 1992

- EnergyWise Homes plans to increase public awareness through seminars, radio, and television. The organization informed real estate agents about the Ilinois HERS through a direct mail campaign. 


\section{Indiana}

Source of Information: Tony Dzwonar, Program Manager, Office of Energy Policy, 317/232-8979

\section{Recent Developments}

In March 1992, the Indiana Department of Commerce, Office of Energy Policy (OEP) convened a Steering Committee to develop a market-driven, statewide, voluntary HERS linked with EEMs. Modeled after the National HERS/EEMs Collaborative, the 30-member Steering Committee included representation of a broad range of stakeholders, including academic, appraiser, builder, consumer, subcontractor, state government, home inspector, HVAC supplier, lender, real estate agent, and utility interests. Funds from the canceled Residential Conservation Service (RCS) program (originally contributed by utilities) as well as federal grant money finance the Steering Committee's work.

The Steering Committee is divided into four Technical Subcommittees:

(1) The Technical Subcommittee-rating tool issues (e.g., what should be rated, training issues, and quality control)

(2) The Marketing Subcommittee-materials and a strategy for marketing the HERS and EEMs to industry and consumers

(3) The Program Organization Subcommittee-an institutional infrastructure for administering a HERS program linked with EEMs, and

(4) The EEMs Subcommittee-educational materials for lenders on incorporating the new energy ratings into EEMs.

The Steering Committee is meeting monthly through 1992 and 1993 . Following the NationalCollaborative's example, they have sought, through an informal process of open and frank discussions, to reach consensus. The OEP contacted committee members prior to the first meeting to familiarize them with the purpose and goals of the HERS/EEM project. At the first meeting, OEP conveners told the committee that broad-based support and cooperation would be needed if the linkage between the HERS and EEMs was going to succeed. All involved have a stake in the outcome and have worked to create a viable process.

The OEP recently released a solicitation for bids from contractors to supply the state with HERS software and training materials. Adopting some of the National Collaborative's recommendations, the announcement specified a HERS that provided accurate estimates and was fuel-neutral. OEP consulted Indiana builders, mortgage lenders and those in the energy rating industry to secure their input and approval before publishing the solicitation. In January 1993, the office had a list of respondents. In June 1993, the OEP contracted with Energy Rated Homes of America (ERHA) to provide HERS software and rater training in Indiana. Energy Rated Homes of Indiana (ERH of IN) will administer the program. OEP expects ERH of IN to begin conducting ratings in fall 1993.

OEP is disseminating a brochure describing the link between HERS and EEMs to lenders, real estate agents, builders, and remodeling contractors.

Once a suitable HERS has been selected, OEP will administer a pilot program to test the viability of the linkage between HERS and EEMs as outlined by the Steering Committee. The program, which aims to accumulate 100 home energy ratings and 100 EEMs, will be confined to one geographic location within 
the state. At the conclusion of the pilot phase, the OEP will evaluate the results and recommend improvements. The OEP expects to extend the improved program to the rest of Indiana.

Although Indiana lenders have been offering EEMs for many years, they have not aggressively marketed them and few borrowers have taken advantage of their availability. EEMs currently available follow the guidelines set forth by Freddie Mac and Fannie Mae. OEP is working with the Indiana Mortgage Bankers Association, the Bankers Association, and the League of Savings Institutions-all members of the Steering Committee-to develop the link between HERS and EEMs. At this time, OEP is only working with the mortgage community on EEMs. However, OEP will provide information on EEMs to any interested party.

\section{Secondary Information}

\section{U.S. Department of Energy Report (1992)}

In March 1992, the Indiana Department of Commerce, Office of Energy Policy, assembled and facilitated an Indiana HERS/EEMs Steering Committee to develop an industry-developed, market-driven, statewide, voluntary HERS program linked with EEMs. The purpose of this initiative is to increase the energy efficiency of new and existing housing in Indiana. The Steering Committee will meet throughout 1992 and 1993 (p. 87).

\section{NASEO Survey (1991)}

Indiana state energy officials are interested in developing a HERS.

\section{Southern Electric International (Good Cents)(1992)}

Northern Indiana Public Service Company has a Good Cents program.

Barakat and Chamberlin, Inc. (1992)

The following utilities have DSM programs:

- Indiana Michigan Power Company

- Knox County Rural EMC

- Northern Indiana Public Service Company

- PSI Energy.

\section{Utility Data Institute (1992)}

The following Indiana utilities have DSM programs:

- Indianapolis Power and Light Company

- Wabash Valley Power Association.

\section{HERS Provider Information Network (Taylor 1993)}

The Indiana Department of Commerce, Office of Energy Policy, Indianapolis, is joining the HERS Provider Information Network. 
PERI (1992)

Steve Baden: The state energy office is collaborating with utilities and industry representatives to develop a HERS in Indiana (10/30/92).

\section{FmHA (1993)}

Indiana building codes/standards meet or exceed those of CABO MEC 89.

\section{Other Sources}

Amy Stewart, Director, Indiana Office of Energy Policy, letter to DOE: Indiana has formed a collaborative to develop a HERS program linked with EEMs (6/11/92). 


\section{lowa}

Source of Information: Craig Stark, Program Planner, Iowa Department of Natural Resources, $515 / 281-4739$

\section{Recent Developments}

In 1990 Iowa passed legislation (S.F. 2403) mandating that a HERS for new homes be implemented by July 1, 1992 and for existing homes, by July 1, 1993. In 1992, the Iowa Department of Natural Resources (DNR), using PVE funds, contracted with Energy Rated Homes of America (ERHA) to develop a HERS program for the state.

In June 1992, representatives from ERHA and Chittenden Bank in Vermont led a training program for Iowa raters and lenders. These representatives and DNR discussed EEMs with two Des Moines mortgage companies and the State Housing Finance Authority. In November 1992, three Des Moines lenders-James Mortgage Corporation, Midland Financial Mortgages, and Iowa Mortgage Corporation-started providing EEMs. As of January 1993, Midland Financial Mortgage had underwritten three EEMs.

Iowa has a mandatory state building code based on the national Model Energy Code. DNR adapted the ERHA software to include state building code inputs for single family homes.

DNR has issued a RFP soliciting bids from prospective Iowa HERS providers interested in training raters, doing ratings, and processing requests for ratings.

DNR has prepared a draft brochure for real estate agents and lenders to distribute to home buyers. Although an Iowa HERS program has been developed, the program has lacked funding and has been minimally promoted.

\section{Lending Institutions Known to Offer EEMs}

- James Mortgage Corporation, Des Moines

- Midland Financial Mortgages, Des Moines

- Iowa Mortgage Corporation, Des Moines.

\section{Secondary Information}

\section{U.S. Department of Energy Report (1992)}

A 1990 Iowa law mandates that the state develop a rating system for new residential buildings by July 1, 1992, and that a rating system for existing residential buildings be established by July 1, 1993 .

The Iowa HERS program is based on the ERHA System. ERHA has adapted the software to Iowa building standards by incorporating MEC inputs, and is providing rater training and technical assistance.

ERHA representatives and staff from the Iowa Division of Natural Resources are meeting with Iowa mortgage firms to discuss EEMs. The James Mortgage Corp. of Des Moines has agreed to offer EEMs (p. 114). 


\section{NASEO Survey (1991)}

Iowa is developing a HERS.

\section{Southern Electric International (Good Cents)(1992)}

The following Iowa utilities have Good Cents programs:

- Cedar Falls Utilities

- Waverly Light and Power.

Barakat and Chamberlin, Inc. (1992)

The following utilities have DSM programs:

- Alton Electric Department

- Denison Municipal Utilities

- Iowa Electric Light and Power

- Iowa Southern Utilities Company

- Iowa - Illinois Gas and Electric Company

- Midwest Gas

- Midwest Power

- Osage Municipal Light and Power Department

- Sanborn Municipal Power and Light

- Sioux Center Electric Department

- United Cities Gas.

Utility Data Institute (1992)

Iowa Public Service Company has a DSM program.

HERS Provider Information Network (Taylor 1993)

The Iowa Department of Natural Resources, Des Moines, is joining the HERS Provider Information Network.

\section{Vories and George (1991)}

The Iowa Department of Natural Resources, Energy Bureau, which is implementing Iowa's HERS program:

- Is phasing in the rating system, beginning with new homes in 1992; by 1996 ERHA of Iowa will be able to perform ratings on all buildings, including commercial/industrial buildings

- Has allocated $\$ 100,000$ for FY 1992; program is expected to be self-supporting once these funds have been expended

- Has not yet determined (in 1991) if the program will be linked with EEMs (p. 89). 


\section{FmHA (1993)}

Iowa building codes/standards meet or exceed those of CABO MEC 89.

\section{Other Sources}

Craig Stark (Iowa Department of Natural Resources) letter to ERHA: The Iowa Department of Natural Resources is implementing a HERS:

- Formed an Iowa Technical Advisory Committee and Steering Committee

- Revised ERHA software to incorporate the Model Energy Code (Iowa's building standard)

- Prepared a draft "Development and Implementation Plan," a "Draft Marketing Plan," and a draft brochure entitled "The Smart Way to Buy or Sell a Home"

- Featured a panel discussion on HERS at the "Iowa's Pursuit of Energy Efficiency Conference" on October 21, 1992

- ERHA held a three-day Energy Raters Training workshop in June 1992. Twenty-one raters and appraisers registered for the workshop. A separate one-day workshop was held for lenders (10/2/92). 


\section{Kansas}

Source of Information: Norma Phillips, Administrator, Weatherization/CSBG Programs, Department of Housing and Commerce, Housing Division, 913/296-2262, and Russell Rudy, Program Consultant, Department of Commerce and Housing, Housing Division, 913/296-2065

\section{Recent Developments}

In 1992, the Kansas Corporation Commission awarded a $\$ 20,000$ grant to the Department of Commerce and Housing, Housing Division (HD) to research HERS programs. HD contracted with the Kansas State University Engineering Extension Service (KSU-EES) to review a variety of rating systems, including Energy Rated Homes of America (ERHA), the California Home Energy-Efficiency Rating System (CHEERS), and Good Cents programs. In addition, HD has contacted program managers with ERH of Alaska, ERH of Virginia, Austin Energy-Star, and El Paso Power and Light's Discovery Home program. For each program, KSU-EES will evaluate:

- The type of audit

- The system used for rating homes

- How the program is administered

- The differences between state, non-profit, and utility HERS programs.

HD expects the report to be completed by early July 1993. After a review of the recommendations in the report, HD staff expects to collaborate with other interested parties to recommend policies for implementation of a HERS plan.

HD staff members currently are collaborating with utilities to collect data on the energy efficiency of insulating materials in two test houses. Plans for the future include collecting data on the energy efficiency of mechanical systems of additional test houses and publication of the results of the data collected on the test homes. In addition, HD staff hope to work towards linking affordability and energy efficiency in public housing.

\section{Secondary Information}

\section{U.S. Department of Energy Report (1992)}

In FY 1993, the Kansas Department of Commerce and Housing and the Energy Programs Section of the Kansas Corporation Commission plans to conduct an educational program on HERS for building industry leaders. The purpose of the program is to acquaint contractors, developers, financial institutions, lenders, realtors and others directly involved in the home building industry with HERS, its potential benefits to home owners, and to energy conservation. Kansas hopes that the educational program will be followed by a serious attempt to formally implement a HERS program (p. 114).

\section{NASEO SUrvey (1991)}

State energy officials are interested in developing a HERS. They cite the lack of resources as a barrier. 
Barakat and Chamberlin, Inc. (1992)

The following utilities have DSM programs:

- Entex

- Kansas Electric Power Cooperative, Inc.

- Kansas Gas and Electric Company

- Kansas Power and Light Company

- Kaw Valley Electric Cooperative Company

- Midwest Energy, Inc.

HERS Provider Information Network (Taylor 1993)

The Kansas Department of Commerce and Housing, Topeka, is joining the HERS Provider Information Network. 


\section{Kentucky}

No primary information available.

\section{Secondary Information}

\section{U.S. Department of Energy Report (1992)}

Kentucky is considering implementing a HERS program.

The Kentucky Division of Energy (DE) recently launched a program-the Energy Saving Index (ESI)-to increase public awareness of and market demand for highly energy-efficient homes, both new and retrofit. The DE calculates and publishes a daily ESI representing the amount of energy that would be saved by owning an energy-saving home compared with consumption of a standard home (p. 64).

\section{NASEO Survey (1991)}

Kentucky state energy officials want to develop a HERS and are considering the Energy Rated Homes of America system.

Barakat and Chamberlin, Inc. (1992)

The following utilities have DSM programs:

- Bowling Green Municipal Electric Division

- East Kentucky Power Cooperative, Inc.

- Kentucky Utilities Company.

\section{Utility Data Institute (1992)}

The following Kentucky utilities have DSM programs:

- Big Rivers Electric Corporation

- Louisville Gas and Electric Company.

\section{HERS Provider Information Network (Taylor 1993)}

The Kentucky Department of Natural Resources, Frankfort, is joining the HERS Provider Information Network.

\section{Vories and George (1991)}

The Kentucky Division of Energy has completed the pre-planning phase of HERS program development. Program sponsors expect that the FY 92 State Energy Conservation Plan will include a feasibility study of the state's HERS program.

- Implementation of the Kentucky HERS program depends on the results of the feasibility study (conducted during late 1991/early 1992) 
- Budget allocation for HERS feasibility study is $\$ 10,000$ to $\$ 15,000$

- Linkage with EEMs is under consideration (p. 91).

FmHA (1993)

Kentucky building codes/standards meet or exceed those of CABO MEC 89. 


\section{Louisiana}

Source of Information: Diane D. Smith, Director, Energy Division, Department of Natural Resources, 504/342-2133, and Wade Byrd, State Energy Conservation Program Supervisor, Energy Division, Department of Natural Resources, 504/342-3476

\section{Recent Developments}

In 1990, the Louisiana Department of Natural Resources (DNR), Energy Division (ED) began exploring HERS programs in response to a DOE requirement that recipients of State Energy Conservation grants develop residential energy conservation programs. In 1991, the division received state authorization for a HERS after the Louisiana Joint Legislative Oversight Committee on Oil Overcharge Monies approved a proposal for a HERS program submitted by DNR. However, lack of staff and conflicting program priorities prevented the department from taking further action on a HERS until 1992, when DNR began to research the Energy Rated Homes of America (ERHA) system. ED contacted two ERH HERS providers-ERH of Arkansas and ERH of Alaska-and, in early 1993, decided to use the ERHA system in Louisiana.

By late January 1994, ED expects an ERHA HERS program to be in place. The program will most likely be administered in-house. Under this arrangement, Louisiana would support the HERS program with PVE funds for approximately three years. During this time, DNR would deposit all program income-such as rating fees and utility contributions-in a separate account. The program would begin drawing on these funds after exhausting state-allocated monies.

Wade Byrd, State Energy Conservation Supervisor, included the HERS/EEM plan in Louisiana's State Energy Conservation Program/Energy Extension Service Plan which was approved by DOE's Regional Office in Dallas in July 1993. DNR staff have informally met with representatives from Cajun Electric, GSU, and Entergy to discuss utility involvement in linking the HERS program with EEMs. Lenders and real estate agents have not yet been approached.

In early December 1993, DNR plans to convene representatives from gas and electric utilities; the mortgage, banking, building (including retrofit), and real estate industries, and the Louisiana university system to:

- Introduce participants to HERS and EEMs

- Solicit input on how to implement a HERS program linked with EEMs, and

- Determine the degree to which each party is willing to support and participate in linking HERS and EEMs.

Pending a favorable response from group members, DNR will form an Advisory Board which will counsel ED on the implementation of Louisiana's initiative to link a HERS with EEMs.

In mid-April 1993, a bill mandating implementation of a linkage between HERS and EEMs in Louisiana was introduced in the state legislature but did not pass. 


\section{Secondary Information}

\section{U.S. Department of Energy Report (1992)}

Does not have a statewide HERS program (p. 102).

\section{NASEO Survey (1991)}

Louisiana has a HERS based on the ERHA system and state officials have had a positive experience with the rating system. The rating system is not linked with EEMs. Energy officials cite lack of resources to properly promote and manage the program as a barrier.

\section{Southern Electric International (Good Cents)(1992)}

The following utilities in Louisiana have Good Cents programs:

- Dixie Electric Cooperative

- Southwestern Electric Power Company.

\section{Barakat and Chamberlin, Inc. (1992)}

The following utilities have DSM programs:

- Cajun Electric Power Cooperative

- Entex

- Valley Electric Membership Corporation.

\section{Utility Data Institute (1992)}

Southwestern Electric Power Company has a DSM program.

\section{HERS Provider Information Network (Taylor 1993)}

The Louisiana Department of Natural Resources, Baton Rouge, is joining the HERS Provider Information Network.

\section{Vories and George (1991)}

1. The Louisiana Department of Natural Resources, Energy Division (SEO) has requested funds for a feasibility study of a Louisiana HERS.

- The SEO is reviewing HERS software

- Eligible structures will be limited to single-family dwellings

- Targeted implementation date is January 1992

- Linkage with EEMs is under consideration (p. 92).

2. The Improved Energy Efficient Home program, administered by the Southwestern Electric Power, Company (SWEPCO):

- Began operating in 1976; program is updated periodically 
- Uses a labeling program based on builders meeting certain energy-efficiency building standards

- Labels new, single-family electric homes

- Has labelled 5,644 homes since 1978

- Has an operating cost of approximately $\$ 120,000$ per year

- Is considering linking the program with EEMS

- May be replaced by the Good Cents Home Program; is interested in a program that would certify rather than just label homes (would help to increase the energy efficiency of homes built at current building standards by $25 \%$ to $50 \%$ )

- Offers a rebate on energy-efficient heat pumps

- Completed a study (in 1991) of 220 homes receiving heat-pump rebates in 1989 (p. 76 ). 


\section{Maine}

No primary information available.

\section{Secondary Information}

\section{U.S. Department of Energy Report (1992)}

The state has appropriated $\$ 130,000$ to develop a home energy rating program. A computer program has been designed and is being tested through the Maine EES Energy Agents Program (p. 9).

\section{Southern Electric International (Good Cents)(1992)}

Central Maine Power Company has a Good Cents program.

Barakat and Chamberlin, Inc. (1992)

The following utilities have DSM programs:

- Bangor Hydro-Electric Company

- Central Maine Power Company

- Kennebunk Light and Power District

- Maine Public Service Company.

\section{HERS Provider Information Network (Taylor 1993)}

The Maine Department of Economic and Community Development, Augusta, is joining the HERS Provider Information Network. 


\section{Maryland}

Source of Information: David Cronin, Energy Resources Officer, Maryland Energy Administration, 410/974-3751; Monica Schmidt, Director of Marketing, Southern Maryland Electric Cooperative (SMECO), 301/932-1450; and Mike Rubala, Marketing Energy Services, SMECO, 301/274-4338

\section{Recent Developments}

In 1991, Maryland passed legislation (H.B. 520-1991) requiring utilities to develop conservation programs under the authority of the Public Service Commission (PSC). PSC directed all of Maryland's utilities to form collaborative teams that included representatives from the Maryland PSC, the Office of People's Counsel, the Maryland Department of Natural Resources, the Maryland Energy Administration, environmental and public interest groups, and major customers to develop energy conservation programs. The larger private utilities-Potomac Electric Power Company (PEPCO), Baltimore Gas and Electric (BG\&E), Delmarva Power and Light, Washington Gas, and Potomac Edison-began their efforts first, followed by rural cooperatives, municipal electric and gas companies. Approximately fifteen of Maryland's twenty utilities have collaborative teams in place. By the end of 1993, the Maryland Energy Administration (MEA) expects all utilities to have energy conservation programs in place or under development.

The structure and organization of the collaborative teams vary by utility. Most teams strive to reach decisions by consensus. As of April 1993, Maryland's four largest utilities have either implemented or have filed programs with the PSC for new home construction. Two utilities-PEPCO and BG\&E-have filed program plans for existing homes. Both plan to develop energy rating programs for new homes. Most programs will involve rebates for new home construction. Utilities operating retrofit programs, such as BG\&E, offer rebates for weatherization and the installation of energy-efficient appliances. Energyefficiency standards vary by utility and are based on a utility's avoided cost.

PSC must approve all utility conservation programs. PSC has recommended that utilities link their programs with EEMs. Utility programs incorporating EEMs must be reviewed by Fannie Mae, Freddie Mac, FHA, and the DVA to determine if they comply with EEM guidelines. As of April 1993, approximately four utilities were pursuing EEMs.

The MEA expects to develop a statewide HERS once the utility programs are in place. The state does not have a current building code in force. All counties must adhere minimally to BOCA 89 standards. Compliance with a higher standard is voluntary.

In April 1993, Southern Maryland Electric Cooperative (SMECO) launched a new DSM program-the PowerSaver Home program-linked with EEMs. The PSC has approved the program. SMECO will offer rebates of up to $\$ 3,500$ to purchasers of new electric homes registered in the program. ${ }^{2}$ Fannie Mae, FHA, DVA, and unofficially, Freddie Mac, will allow purchasers to apply the utility rebate to closing costs, the down payment, or reserve funds.

When the program was in the developmental stage, SMECO convened an Advisory Committee and asked several lenders to participate. In January 1993, Helen Souder, a member of the Advisory Committee representing Crestar Bank, contacted a Fannie Mae representative about SMECO's new program and persuaded them to back EEMs linked with homes registered in the PowerSaver Home program.

\footnotetext{
${ }^{2}$ Homes with dual fuel heating systems which have electricity as the dominant energy source also are eligible.
}

Maryland 
As of April 1, 1993, 43 homes have been registered in the PowerSaver Home program. To be eligible for PowerSaver Home rebates, new homes must be built by participating builders who meet the following requirements. Builders must:

- Sign an agreement stating that they will abide by SMECO's energy-efficiency construction standards

- Register all new homes prior to laying the foundation. SMECO will review the plans to make sure the utility's standards are being met. SMECO employees will conduct on-site construction inspections, including an inspection of the completed home

- Use a heating contractor who is participating in SMECO's rebate program.

SMECO is taking an active role in training lenders, real estate agents, builders, and heating contractors. In February and March 1993, the utility sponsored a seminar for builders. Seminars for real estate professionals and appraisers were scheduled for April. SMECO staff are also developing a course for real estate agents that may be applied towards accreditation. Seminars for buyers in SMECO's service territory were planned for late May 1993.

\section{Lending Institutions Known to Offer EEMs}

Crestar Bank.

\section{Secondary Information}

\section{U.S. Department of Energy Report (1992)}

Does not have a statewide HERS program (p. 47).

\section{Barakat and Chamberlin, Inc. (1992)}

The following utilities have DSM programs:

- Baltimore Gas and Electric Company

- Delmarva Power and Light Company

- Potomac Edison Company

- Potomac Electric Power Company

- Southern Maryland Electric Cooperative, Inc.

- Washington Gas Light Company.

\section{HERS Provider Information Network (Taylor 1993)}

The following organizations are joining the HERS Provider Information Network:

- Maryland Energy Administration, Annapolis

- Baltimore Gas and Electric, Baltimore

- Energy Conservation Management, Inc., Baltimore

- Conservation Management Corporation, Bethesda. 
PERI (1992)

Steve Baden: Cited state-level efforts to develop collaboration between government, utilities, and industry for development of HERS in Maryland (10/30/92).

FmHA (1993)

Maryland building codes/standards meet or exceed those of CABO MEC 89.

\section{Other Sources}

In the Strategic Energy Plan for Maryland the Governor's Energy Task Force (1993) recommended that the state implement an energy-efficient home mortgage pilot program. Citing the National HERS/EEMs Collaborative's finding that the lack of data quantifying the impact of EEMs was an impediment to the general availability of EEMs, the Governor's Task Force proposed that the state of Maryland participate in pilot projects aimed primarily at quantifying the impact of mortgages on the quality of the portfolios in the secondary mortgage market.

Opportunities for such involvement exist under existing budget authorities of various federal agencies, under the provisions of current legislation pending before Congress, and with the support of the National HERS/EEMs Collaborative. The Governor's Task Force outlined a short-term implementation strategy:

- Identify a coordinator from the Department of Housing and Community Development (DHCD) to lead Maryland's efforts to be a project state, and

- Establish contacts with (1) appropriate officials from the states of Wisconsin, Vermont and other states taking leadership roles in this area, (2) Alliance to Save Energy personnel involved in the energyefficient mortgage process, and (3) the DOE collaborative on energy-efficient mortgages, to determine what steps must be taken by Maryland to be identified as a pilot project locale.

The Governor's Task Force released its recommendations prior to HUD's announcement of the states selected to participate in the HUD/FHA EEM Pilot Program. Maryland had hoped to be one of the five states selected. 


\section{Massachusetts}

No primary information available.

\section{Secondary Information}

\section{U.S. Department of Energy Report (1992)}

The Massachusetts Division of Energy Resources is researching HERS programs (p. 11).

Barakat and Chamberlin, Inc. (1992)

The following utilities have DSM programs:

- Bay State Gas Company

- Boston Edison Company

- Chicopee Electric Light Department

- New England Electric System

- North Attleboro Electric Department

- Northeast Utilities

- Reading Municipal Light Department

- Taunton Municipal Lighting Plant.

HERS Provider Information Network (Taylor 1993)

Western Massachusetts Energy Crafted Homes, Boston, is joining the HERS Provider Information Network.

\section{Vories and George (1991)}

In 1990, a consortium of Massachusetts, Rhode Island, and New Hampshire electric utilities launched the Boston-based Energy Crafted Homes of New England program.

- Program goal is to reduce energy use by $50 \%$ relative to the Massachusetts Energy Code

- Consortium developed computer software to evaluate energy performance of homes. Provides software and training to builders

- Each utility certifies homes in their territory

- Eligible structures include new, single-family and multifamily residences; program is fuel neutral

- Annual budget is approximately $\$ 2$ million

- Program not directly linked with EEMs; however, utilities provide builders and home buyers with a list of lenders who offer EEMs (p. 57).

PERI (1992)

Steve Baden: Massachusetts is developing legislation to create a HERS (10/30/92). 


\section{Michigan}

Source of Information: Rose Hughes, Program Manager, Energy Resources Division, Michigan Public Service Commission, 517/334-7235; Jerry Nash, Building Construction Specialist, Office of Energy, Michigan Public Service Commission, 517/334-7236; and Lloyd Walker, Weatherization Manager, EightCAP, Inc., 616/754-9315

\section{Recent Developments}

In July 1992, EightCAP, Inc., a community group in Greenville, Michigan, received a $\$ 40,000$ grant from the Michigan Public Service Commission (MPSC) to conduct 500 home energy ratings in Greenville. The MPSC awarded the one-year grant to test the feasibility of administering a HERS program through local networks.

The MPSC reviewed two software packages for the Energy Rating System Pilot (ERSP)-PEAR (Program for Energy Analysis of Residences) and ARES - and selected the former for use in the pilot. The PEAR system, based in part on the Michigan energy code (ASHRAE 90 A.80), was easy to use and could be adapted to perform ratings on existing homes. The MPSC provided EightCAP, Inc. with both the software and training necessary to conduct the ERSP.

Between September 1992 and January 1993, EightCAP, Inc. sent approximately 1600 letters to Greenville. homeowners promoting free home energy ratings for both new and existing homes. Only 129 homeowners responded. EightCAP, Inc. had expected that the mailings and publicity would generate greater interest from the public. EightCAP and MPSC project coordinators speculate that the primary reason for the low response level was that the promotional literature did not specify how much could be saved on energy bills if a home was energy-efficient.

The 129 homeowners participating in ERSP received a certificate rating the energy efficiency of their homes on a scale of one to 10 and a letter explaining what the rating meant. The letter also notified homeowners that further information on how to improve a home's energy efficiency was available upon request. EightCAP, Inc. had hoped to include information on EEMs in the follow-up material; however, very few requested the additional information.

In May 1993, EightCAP, Inc. sent out a final mailing to Greenville homeowners. EightCAP, Inc. will begin distributing the ERSP promotional literature in areas outside the Greenville city limits in summer 1993. EightCAP, Inc. plans to send additional information on the dollar savings attached to energyefficient appliances to the 129 homeowners whose homes were rated.

The MPSC may extend the ERSP October 1993 deadline should EightCAP, Inc. request the additional time. The MPSC may launch a statewide program sometime in the future, depending on the availability of funds and the establishment of national HERS guidelines.

\section{Establishing a Linkage with EEMs}

The link between HERS and EEMs is an integral component of the ERSP. In June 1992, FMB Commercial Bank of Greenville pledged support for the pilot program. The bank was in the process of contacting Freddie Mac and had hoped to begin offering EEMs in early 1993. By the end of the year, FMB reported to EightCAP, Inc. staff that the process was more complicated than anticipated, that the bank's master contract with Freddie Mac did not allow EEMs, and that they had lost interest in the program. 
After some additional discussion in late May 1993, FMB took renewed interest in the project and amended their master contract with Freddie Mac to allow EEMs. During summer 1993, the bank will promote EEMs with EightCAP, Inc. through its advertising and meetings with Montcalm Board of Realtors members. The bank may also ask its appraisers to accept a specific PEAR rating as designation of an energy-efficient home.

EightCAP, Inc. is revising its promotional literature to emphasize the availability of EEMs for financing energy-efficiency improvements.

\section{Lending Institutions Known to Offer EEMs}

FMB Commercial Bank, Greenville.

\section{Secondary Information}

\section{U.S. Department of Energy Report (1992)}

The Michigan Public Service Commission (MPSC) awarded a $\$ 40,000$ grant to EightCAP to pilot a HERS in western Michigan. MPSC is providing the HERS software (the PEAR program) and rater training. EightCAP will perform ratings on 500 or more homes. The agency is also exploring a linkage between HERS and EEMs and is meeting with local banks (p. 90).

Barakat and Chamberlin, Inc. (1992)

The following utilities have DSM programs:

- Consumers Power Company

- Croswell Municipal Light and Power Department

- Detroit Edison Company

- Lansing Board of Water and Light

- Traverse City Light and Power Department.

Utility Data Institute (1992)

Michigan Gas Utilities runs a DSM program.

HERS Provider Information Network (Taylor 1993)

The Michigan Energy Resources Division, Lansing, is joining the HERS Provider Information Network.

\section{Other Sources}

Letter from Rose Hughes, Energy Resources Division, Michigan Public Service Commission, to Barbara Farhar (NREL): The following Michigan utilities also have DSM programs:

- Michigan Consolidated Gas Company

- Indiana - Michigan Power Company

- Michigan Gas Company (7/26/93). 


\section{Minnesota}

Source of Information: Bruce Nelson, Senior Engineer, Energy Technology Unit, Energy Division, Minnesota Department of Public Service, 612/297-2313; Michael Roelofs, Manager, Contracts and Grants Administration Unit, Energy Division, Minnesota Department of Public Service, 612/297-2545; Barbara Delaney, Product Development Consultant, Northern States Power (NSP), 612/330-6858; and Duane Erlien, Residential Marketing Manger, Minnegasco, 612/372-4664

\section{Background}

Minnesota has a stringent state energy building code. Only a few state residents take advantage of EEMs and there is no effort to promote the availability of EEMs.

Approximately 10 years ago, the Minnesota Department of Public Service (DPS) sponsored a series of focus groups involving lenders, builders, home purchasers, contractors, and real estate agents in the Twin Cities area to determine the level of interest in HERS. DPS determined that focus group members did not express enough interest to merit the development of a Minnesota HERS.

\section{Recent Developments}

In 1992, the DPS directed Northern States Power (NSP) - a combined utility - to work with at least one gas utility on a new residential construction program. In late 1992, NSP Electric and NSP Gas contacted Minnegasco (a gas utility). In February 1993, the two utilities began collaborating to develop a program for the energy-efficient construction of new homes. NSP has since discontinued its Performance Plus program, which served electrically-heated, new homes.

In October 1992, prior to joining NSP's initiative, Minnegasco had begun to develop its own certified energy-efficient home program. The utility held meetings with representatives from the state government and legislature, builders, contractors, and homeowners to solicit comments on its proposed program. It was during this time that NSP first approached Minnegasco about a joint initiative.

Between February and April 1993, NSP and Minnegasco representatives reviewed existing certification programs, such as Energy Crafted Homes, Good Cents, and R-2000. In May 1993, NSP Electric and Minnegasco began work on the certification tool and program design.

The new construction certification program will:

- Strive to consider the overall performance of the house and its utility costs rather than assessing the effectiveness of individual energy-efficiency measures. The program will require, among other things, the use of energy-efficient appliances and controls, a mechanical ventilation system, and energyefficient lighting

- Be fuel neutral

- Certify only new construction

- Be available to customers in the utilities' service territories (initially, in the St. Paul/Minneapolis metropolitan area)

- Be linked with EEMs. Details will be worked out between July and September 1993 
- Offer incentives/rebates to encourage customers and builders to install energy-efficiency features in their homes.

Homes certified by the program must comply with standards established by the utilities. Utility representatives will inspect completed homes before certifying them.

NSP representatives have worked with staff-who have expertise in the Minnesota Building Code-at the Cold Climate Housing Center at the University of Minnesota. They also have held discussions with the Builders Association of Minnesota regarding NSP's upcoming educational program to introduce builders and building code officials to residential energy-efficiency technologies and practices. The program will begin full operation in 1994.

\section{Secondary Information}

\section{U.S. Department of Energy Report (1992)}

Does not have a statewide HERS program (p. 93).

\section{NASEO SUrvey (1991)}

Minnesota is developing a HERS. Lack of resources and uncertainty about the accuracy of the rating system were cited as barriers.

\section{Barakat and Chamberlin, Inc. (1992)}

The following utilities have DSM programs:

- Aitkin Public Utilities Commission

- Anoka Electric Cooperative

- Dakota Electric Association

- Detroit Lakes Public Utility

- Lake Region Cooperative Electric Association

- Litchfield Public Utility Commission

- Marshall Municipal Utilities

- Midwest Gas

- Minnesota Power and Light

- Moorhead Public Service Department

- New Ulm Public Utilities Commission

- Northern States Power Company

- Ortonville Water and Light Department

- Otter Tail Power Company

- Owatonna Public Utilities

- Rochester Public Utility Department

- Waseca Water and Light Department

- Willmar Municipal Utility Commission. 


\section{Utility Data Institute (1992)}

The following Minnesota utilities have DSM programs:

- Cooperative Power Association

- Minnegasco

- Minnesota Power Company.

HERS Provider Information Network (Taylor 1993)

The following organizations are joining the HERS Provider Information Network:

- Minnesota Energy Division, St. Paul

- Minnegasco, St. Louis Park.

FmHA (1993)

Minnesota building codes/standards meet or exceed those of CABO MEC 89. 


\section{Mississippi}

Source of Information: Wes Miller, Senior Contract Manager, Mississippi Department of Economic and Community Development, 601/359-6600

\section{Background}

In 1992, the Mississippi Community Development Block Grant (CDBG) program received funds from the HUD Housing Investment Partnership Program (HIPP) to implement the state's Comprehensive Housing Affordability Strategy. The goal of the strategy is to increase the quality, affordability, and energyefficiency of low-income housing in Mississippi. HIPP supports low-income home construction and renovation. Housing improvements financed by the program must comply with the energy-efficiency standards of the Model Energy Code (MEC) for new construction and HUD rehabilitation standards for existing homes.

The Mississippi Department of Economic and Community Development, Energy Division (ED), is collaborating with the CDBG program to implement the energy-efficiency standards of the HIPP for lowincome housing. The division is working to link a voluntary, uniform, market-driven HERS program with EEMs to enable state residents who do not normally qualify for a mortgage to become first-time home buyers. The HIPP grant will cover the cost of conducting home energy ratings on low-income homes to determine if they are in compliance with MEC and HUD standards. ED hopes that the HERS/EEMs. program will encourage those in higher income brackets to buy energy-efficient homes or make energyefficiency improvements to their present homes.

\section{Developing a HERS}

In October 1992, ED negotiated a contract with Energy Rated Homes of America (ERHA) to provide the state with HERS software, training materials, and technical assistance. During 1993, ERHA will sponsor two sessions to train and certify raters. The first of these occurred in July in Jackson, Mississippi. Individuals from around the state with a variety of shelter-related experiences attended the course. ERHA plans to hold a second training session in fall 1993. During summer 1993, ED in collaboration with ERHA convened a Technical Advisory Committee of representatives from utilities, lending institutions, lending agencies, real estate agencies, and construction organizations to formulate a nonprofit corporation that would administer the HERS/EEMs program. ED anticipates that incorporation of the nonprofit will be completed by December 1993.

ED hopes to execute HERS training agreements with the Mississippi Department of Labor Assistance and the Department of Human Services' Weatherization Program. No time frame or details have been worked out. ED also hopes (by 1994) to involve Mississippi utility companies in HERS by encouraging them to incorporate the HERS into their integrated resource and DSM programs. Most utilities have their own certification programs (Gas Mark, E-3, and Good Cents programs) and ED speculates that they will need some form of incentive to become involved in a uniform, statewide HERS. Full details of utility participation have not yet been defined.

\section{Establishing a Linkage Between HERS and EEMs}

ED has just begun to interest lenders in EEMs. In collaboration with the state's Agricultural Cooperative Extension Program Offices and ERHA, ED is planning regional meetings with lenders, appraisers, real estate agents, and builders during 1993. The meetings also will involve representatives of the Farmers Home Administration, HUD, and the Department of Veterans Affairs. The conveners will discuss underwriting procedures for EEMs and gauge lender receptivity to voluntarily offering EEMs at below- 
market interest rates as an incentive to borrowers. Prior to the meetings, announcements will be disseminated to the offices and local associations of prospective meeting participants.

\section{Secondary Information}

\section{U.S. Department of Energy Report (1992)}

The Mississippi Department of Economic and Community Development, Energy Division, which is the state energy office, is collaborating with the state's Community Development Block Grant Program to implement a link between HERS and EEMs. Mississippi is licensed to use the ERHA system. Meetings are being scheduled with local primary and secondary lenders to standardize the terms of EEMs (p. 66).

\section{NASEO Survey (1991)}

Mississippi has a HERS based on the Energy Rated Homes of America system. The rating system is linked with EEMs. State energy officials cite the lack of national uniform EEM guidelines as a barrier.

\section{Southern Electric International (Good Cents)(1992)}

The following Mississippi utilities have Good Cents programs:

- Coahoma Electric Power Association

- Coast Electric Power Association

- Delta Electric Power Association

- Dixie Electric Power Association

- Magnolia Electric Power Association

- Mississippi Power Company

- Pearl River Valley Electric Power Association

- South Mississippi Electric Power Association

- Southern Pine Electric Power Association

- Southwest Mississippi Electric Power Association

- Twin County Electric Power Association

- Yazoo Valley Electric Power Association.

\section{Barakat and Chamberlin, Inc. (1992)}

The following utilities have DSM programs:

- Entex

- Four County Electric Power Association.

HERS Provider Information Network (Taylor 1993)

Energy Rated Homes of Mississippi, Jackson, is joining the HERS Provider Information Network.

Vories and George (1991)

The Mississippi Department of Economic and Community Development, Energy and Transportation Division (SEO) is developing a HERS: 
- Has adapted ERHA software (0-to-100 point scale; translates into a Five-Star rating)

- Will perform ratings on new and existing single- and multifamily dwellings

- Has not yet determined target date for implementation (p. 93). 


\section{Missouri}

No primary information available.

\section{Secondary Information}

\section{U.S. Department of Energy Report (1992)}

Does not have a statewide HERS program (p. 121).

\section{NASEO SUrveY (1991)}

Missouri is interested in developing a HERS. Lack of resources and information were cited as barriers.

Barakat and Chamberlin, Inc. (1992)

The following utilities have DSM programs:

- Arkansas Power and Light Company

- Central Electric Power Cooperative

- Kansas City Power and Light Company

- St. Joseph Light and Power Company

- White River Valley Electric Cooperative, Inc.

\section{Utility Data Institute (1992)}

Empire District Electric Company runs a DSM program.

\section{HERS Provider Information Network (Taylor 1993)}

Energy Rated Homes of Missouri, Mountain View, is joining the HERS Provider Information Network.

\section{Vories and George (1991)}

In 1982, Kansas City Power and Light launched the Save America's Valuable Energy (SAVE) program, co-sponsored by the local Home Builders Association.

- Uses prescriptive guidelines; rating is self-certified by the builder

- Limits eligible structures to new, electrically heated homes (gas-fueled homes can participate through the local Home Builders Association)

- Has certified approximately 1500 homes (20\% of new, single-family homes)

- No charge for certification

- Advertised EEMs through the Home Builders Association; no data collected on the number of people who have taken advantage of EEMs through the SAVE program

- Plans to supplant SAVE with the "Total Electric Energy Efficiency Home" program (p. 67). 


\section{Montana}

Source of Information: Louise Moore, Information Team Leader, Conservation and Renewable Energy, Department of Natural Resources and Conservation, 406/444-6699

\section{Recent Developments}

In 1992, Pacific Power and Light (PP\&L) and the Montana Power Company suggested that the Environmental Quality Council (EQC) form a collaborative-the EQC Residential Energy Efficiency Working Group (REEWG) - to resolve a controversy surrounding residential energy efficiency and the state building code. The energy efficiency standards established by the code have been controversial since 1983 when the Northwest Power Planning Council encouraged the states of Montana, Idaho, Oregon, and Washington to adopt the Northwest Energy Code for electrically heated homes. The energy-efficiency standards of the Northwest Energy Code are more stringent than those of Montana's building codes. Montana's investor-owned utilities-Montana Power Company, PacifiCorp, Montana Dakota Utilities, and Great Falls Gas Company-and the Bonneville Power Administration funded the work of the REEWG.

The REEWG was open to all interested individuals and included utilities, home builders, home building suppliers, mortgage lenders, architects and engineers, environmental and low-income groups, local governments, and state and federal agencies. The REEWG met from July 1992 through December 1992, and recommended unanimously that the state of Montana:

- Initiate development and/or selection and testing of HERS applicable to new and existing residences. Subject to available funding, the system will be developed over the next two years. (Montana state energy officials have researched HERS programs and contacted states with HERS programs in place; however, no further action will be taken until national guidelines have been issued and/or funding is available.)

- Provide education, training, technical assessment, and demonstration programs for consumers, builders, building code officials, home inspectors, bankers, real estate agents, and appraisers. REEWG recommended that the Montana state government, housing industry, and utilities collaborate to sponsor these programs

- Require that a label describing the energy-efficiency features (for example, heating appliance efficiencies and ceiling, wall, floor, window, and door R- or U-values) be affixed to the breaker box of a new site-built, manufactured, or modular home

- Support a real estate and housing industry petition to the Federal Housing Administration calling for an increase in the cap or upper limit on FHA home mortgages

- Initiate an EEMs program for first-time home buyers through the Montana Board of Housing (MBH). The program would require a low down payment $(3-5 \%)$ and raise the mortgage ceiling above the current FHA caps. Indemnification for MBH risk exposure for the loan amount above the FHA cap would be assumed in some manner by utilities, state government, or a non-profit entity

- Encourage utilities to offer incentive programs to purchase energy efficiency.

Montana really passed legislation pertaining to EEMs which does not define the actual measures required for an EEM under the Montana Board of Housing Program. 


\section{Lending Institutions Known to Offer EEMs}

Montana Board of Housing.

\section{Secondary Information}

\section{U.S. Department of Energy Report (1992)}

Does not have a statewide HERS program (p. 131).

\section{NASEO Survey (1991)}

Montana is developing a HERS. Uncertainty about the accuracy of the rating system was cited as a barrier.

\section{Southern Electric International (Good Cents)(1992)}

The following Montana utilities have Good Cents programs:

- Flathead Electric Cooperative, Inc.

- Glacier Electric Cooperative, Inc.

- Lincoln Electric Cooperative-Missoula

- Missoula Electric Cooperative, Inc.

- Montana Power Company

- Ravalli County Electric Cooperative

- Vigilante Electric Cooperative.

\section{Bonneville Power Administration (Super Good Cents)(1993)}

The following Montana utilities have Super Good Cents programs:

- City of Troy Power and Light Department

- Flathead Electric Cooperative

- Glacier Electric Cooperative

- Lincoln Electric Cooperative

- Mission Valley Power

- Missoula Electric Cooperative

- Montana Power Company

- Ravalli County Electric Cooperative

- Vigilante Electric Cooperative.

Barakat and Chamberlin, Inc. (1992)

The following utilities have DSM programs:

- Black Hills Corporation

- Bonneville Power Administration

- Great Falls Gas Company

- Montana Power Company

- Montana-Dakota Utilities Company. 


\section{HERS Provider Information Network (Taylor 1993)}

The Montana Department of Natural Resources and Conservation, Helena, is joining the HERS Provider Information Network.

PERI (1992)

Steve Baden: The Montana State Energy Plan includes provisions for the development of a HERS (10/30/92).

\section{Other Sources}

Louise Moore letter (and enclosure) to Barbara Farhar: The Montana Department of Natural Resources and Conservation is participating in the Environmental Quality Conservation (EQC) Residential Energy Efficiency Working Group. The purpose of the collaborative is to:

1. Identify and explore issues related to residential energy efficiency

2. Develop and provide to the EQC consensus recommendations for (a) Montana state policy on the energy efficiency of residential structures and (b) strategies for implementing the policy

3. Make recommendations to other entities concerning the policy implementation strategies, as appropriate.

The EQC Working Group will adhere to a consensus process, outlined in the "ground rules" established by the group. Working Group meetings and those of its subcommittee are open to the public.

The Working Group planned to deliver its recommendations to the EQC by November $15,1992(8 / 25 / 92)$.

Javier Figueroa, Program Manager, Lender/EEMs, Washington State Energy Office, letter (and enclosures) to Barbara Farhar: Montana participates in the MHAP program (4/24/92). ${ }^{3}$

James Nybo, Conservation Analyst, Northwest Power Planning Council, letter (and enclosure) to Barbara Farhar: Montana is participating in the NW Regional Energy-Efficient Mortgage Strategy $(5 / 6 / 92) .^{4}$

${ }^{3}$ See Idaho for full description.

${ }^{4}$ See Idaho for full description. 


\section{Nebraska}

No primary information available.

\section{Secondary Information}

\section{U.S. Department of Energy Report (1992)}

Does not have a statewide HERS program. In 1991, the Nebraska Energy Office sponsored a meeting to explore HERS and EEMs; however, no further activity resulted (p. 124).

Barakat and Chamberlin, Inc. (1992)

The following utilities have DSM programs:

- Alliance Municipal Electric System

- Central City Light Department

- Clarkson Electric Department

- Lincoln Electric System

- Nebraska Municipal Power Pool

- Nebraska Public Power District.

Utility Data Institute (1992)

Omaha Public Power District has a DSM program.

\section{Vories and George (1991)}

The Nebraska Energy Office assembled a task force of representatives from the building industry, lenders, appraisers, realtors and secondary mortgage markets to explore the feasibility of developing a HERS. In July 1991, the committee was developing a state energy policy which included a recommendation to adopt a HERS (p. 94).

PERI (1992)

Steve Baden: The Nebraska State Energy Plan includes provisions for the development of a HERS (10/30/92). 


\section{Nevada}

No primary information available.

\section{Secondary Information}

\section{U.S. Department of Energy Report (1992)}

The Nevada State Energy Office is developing a HERS pilot program that promotes EEMs (p. 165).

\section{NASEO Survey (1991)}

Nevada is developing a HERS. Lack of information was cited as a barrier.

\section{Southern Electric International (Good Cents)(1992)}

The following Nevada utilities have Good Cents programs:

- Sierra Pacific Power Company

- Wells Rural Electric Company.

\section{Bonneville Power Administration (Super Good Cents)(1993)}

Wells Rural Electric Company has a Super Good Cents program.

Barakat and Chamberlin, Inc. (1992)

The following utilities have DSM programs:

- Nevada Power Company

- Sierra Pacific Power Company

- Southwest Gas Corporation.

\section{Vories and George (1991)}

As part of a long-term goal to upgrade the State Energy Code, the Nevada Office of Community Service (state energy office) is interested in developing a HERS program:

- Office is considering working with major utilities in the state to develop a HERS based on the Good Cents model

- Will focus on rating new construction before extending program to existing structures

- Has not yet decided if EEMs will be a part of the program (p. 95). 


\section{New Hampshire}

No primary information available.

\section{Secondary Information}

\section{U.S. Department of Energy Report (1992)}

New Hampshire established a task force to develop incentives to promote energy efficiency in buildings (p. 19).

\section{NASEO Survey (1991)}

New Hampshire has a HERS in a limited number of communities. New Hampshire uses a utility-based system (Energy Crafted Homes). The rating system is not linked with EEMs.

\section{Barakat and Chamberlin, Inc. (1992)}

The following utilities have DSM programs:

- New England Electric System

- New Hampshire Electric Cooperative, Inc.

Vories and George (1991)

New Hampshire participates in the Boston-based Energy Crafted Homes of New England program (p. 57). ${ }^{1}$

\footnotetext{
${ }^{1}$ See Massachusetts for full description.
} 


\section{New Jersey}

Source of Information: Joe Sullivan, Manager of Engineering and Technical Programs, New Jersey Department of Environmental Protection and Energy, Office of Energy, 609/984-9701; and Maurice Weiss, Energy Specialist, Department of Environmental Protection and Energy, Office of Energy, 609/633-2352

\section{Recent Developments}

The New Jersey Energy Master Plan (EMP), signed by the Secretary for Environmental Protection and Energy in November 1991, contains provisions for the development and implementation of a voluntary statewide HERS program linked with EEMs. The implementation section of EMP, approved in February 1993, recommended the following:

- Assembling representatives from the building, utility, real estate, banking and home financing industries, the Department of Environmental Protection and Energy (DEPE), and other government agencies to develop an EEM mechanism

- Implementing a statewide voluntary, uniform HERS program by:

- Promulgating state regulations establishing a New Jersey HERS

- Developing and marketing a HERS

- Incorporating information on HERS in the Department of Community Affairs (DCA) New Home Warranty (for utilities) and Builder Registration programs. The latter program would allow builders to voluntarily register with the DCA for an energy-efficiency construction rating which could then be used in marketing new homes to consumers. In addition to existing utility rebate programs for builders, the rating would provide an added incentive for builders to construct energy-efficient homes.

The Office of Energy (OE) within DEPE hopes to implement a HERS through the state's utility companies. Several of New Jersey's seven investor-owned utilities already have in place or are developing HERS-type programs and are willing to cooperate with DEPE in developing a uniform HERS for the state. OE has provided the utilities with information on EEMs and will convene a meeting of representatives from the utility sector to address the utilities' concerns about a statewide HERS program.

OE has approached lenders-Fannie Mae, Freddie Mac, and the New Jersey Housing Finance Agency-about underwriting EEMs linked with utility programs. Lenders have expressed guarded interest, withholding a firm commitment until reliable means for assessing the energy-efficient standards used by utility rating/certification programs have been developed.

In 1993. OE plans to assemble major players-builders/developers, public and private banking/lending sources, utilities, real estate professionals, and state government representatives-to develop criteria for a uniform, statewide HERS linked with EEMs.

In late 1992, DEPE contacted HUD requesting that New Jersey be considered for the HUD EEM Pilot Program. In early 1993, the New Jersey state legislature passed a resolution endorsing state involvement in the HUD Pilot Program. New Jersey was not selected to participate in the HUD pilot; however, the state is pursuing other funding opportunities. State budget constraints may delay the development and implementation of a HERS program linked with EEMs. 


\section{Secondary Information}

\section{U.S. Department of Energy Report (1992)}

New Jersey has a HERS (p. 28).

\section{NASEO Survey (1991)}

New Jersey is interested in developing a HERS.

\section{Southern Electric International (Good Cents)(1992)}

The following New Jersey utilities have Good Cents programs:

- Atlantic Electric Company

- Jersey Central Power and Light Company.

Barakat and Chamberlin, Inc. (1992)

The following utilities have DSM programs:

- Atlantic Electric Company

- Jersey Central Power and Light Company

- Metropolitan Edison Company

- New Jersey Natural Gas Company

- Orange and Rockland Utilities, Inc.

- Public Service Electric and Gas Company.

\section{HERS Provider Information Network (Taylor 1993)}

The following New Jersey organizations are joining the HERS Provider Information Network:

- Public Service Electric and Gas Company, Newark

- New Jersey Department of Environmental Protection and Energy, Trenton. 


\section{New Mexico}

Source of Information: Ingrid Kelley, Bureau Chief, Energy Information Service Bureau, Energy Conservation and Management Division, Department of Energy, Minerals and Natural Resources, 505/8277196

\section{Background}

Prior to 1987, the state researched the idea of a HERS linked with EEMs but did not pursue it due to lack of funding. Recently, the New Mexico Energy Office (EO) has begun efforts to educate real estate agents on the subject. EO is collaborating with Santa Fe Community College to sponsor a class on energy efficiency and solar energy for real estate professionals. The New Mexico Real Estate Commission approved the class to count towards accreditation for a real estate license.

The El Paso Electric Company's HERS program serves as a model that could potentially be expanded. However, most New Mexico utilities do not seem to have much interest in a HERS/EEM program.

EO expects the state to adopt the 1992 Model Energy Code in early 1994.

\section{Secondary Information}

\section{U.S. Department of Energy Report (1992)}

The 1991 New Mexico State Energy Policy recommended that the state:

- Develop and implement a HERS

- Promote the use of HUD's guidelines for EEMs

- Develop a performance-based standard for energy-efficient housing.

In response to these policy recommendations, New Mexico:

- Has adopted the 1989 Model Energy Code (effective January 1, 1993)

- Is developing an energy-efficiency standard for state-owned facilities; the core of the standard will be ASHRAE 90.1 .

El Paso Electric Company, which serves the Las Cruces area, conducts home energy ratings within its service territory (p. 104).

\section{NASEO Survey (1991)}

New Mexico energy officials are interested in developing a HERS. They cited lack of resources and information as barriers.

Barakat and Chamberlin, Inc. (1992)

The following utilities have DSM programs:

- Continental Divide Electric Cooperative, Inc.

- El Paso Electric Company 
- Public Service Company of New Mexico

- Texas-New Mexico Power Company.

HERS Provider Information Network (Taylor 1993)

The New Mexico Department of Energy, Minerals and Natural Resources, Santa Fe, is joining the HERS Provider Information Network.

\section{Hughes (1992)}

El Paso Electric Company's (EPECO) New Mexico Division is the first utility to incorporate ERHA's rating system into their residential DSM program.

- EPECO markets the ERHA HERS program under the "Discovery Home" label

- Discovery Home program is linked with EEMs. Forty real estate agents and lenders attended a training session on EEMs. Two local lenders have taken the lead in offering EEMs

- Six local raters completed training and are certified to conduct home energy ratings; 15 homes have been rated during the program's initial period of operation

- EPECO is disseminating promotional information through the utility office and the local home builders association. All residential customers in the service territory will receive information on the program.

\section{FmHA}

New Mexico building codes/standards meet or exceed those of CABO MEC 89. 


\section{New York}

Source of Information: Bob Kelley, Executive Director, NYSE-Star, Ltd., 518/465-3115; Mark Eggers, Energy Conservation Specialist, New York State Energy Office, 518/474-4995; and Jack Bronson, Technology Transfer Specialist, DOE New York Support Office, 212/264-3103

\section{Background}

In early 1991, the New York state legislature allocated $\$ 150,000$ in PVE funds to develop and implement a voluntary, builder-certification program for the construction of energy-efficient homes throughout the state. The New York State Energy Office (NYSEO) in collaboration with the New York State Energy Research and Development Authority (NYSERDA), the New York Builders Association, Inc. (NYSBA), and seven investor-owned utilities established New York State Energy-Star (NYSE-Star) to develop and administer the program. In fall 1991, NYSE-Star was incorporated as a non-profit organization and a Board of Directors was designated. The board is composed of three builders, two energy suppliers/utilities, and two representatives from state government. Although no current plans exist to expand the board, NYSE-Star bylaws allow board members to appoint two additional directors from participating lending institutions, building suppliers, or academic, professional or trade organizations.

The board met during fall 1991 to develop a NYSE-Star construction standard and outline a pilot program. The NYSE-Star standard approved by the board:

- Exceeds the New York State Energy Code by 25 percent

- Incorporates requirements for reduced air infiltration and blower door testing.

In addition, the board decided that new homes, regardless of fuel source used, would be eligible for certification.

In late 1991, NYSE-Star launched a pilot program in New York State Electric and Gas Corporation's southern service territory. Builders interested in the pilot were required to attend a training course on NYSE-Star standards for energy-efficient construction; participants received NYSE-Star certification. Certified builders submitted building plans to NYSEO for inspection and approval, and contractors conducted on-site inspections. New York State Electric and Gas Corporation provided builders of electric homes with a rebate for each NYSE-Star-approved home constructed.

The pilot phase ended in early 1992 with promising results:

- There was strong interest among builders who found that complying with NYSE-Star standards was not difficult

- More utilities became interested in participating in the NYSE-Star program. (Currently, all utilities in New York State are partners in NYSE-Star, financially contribute to the organization, and sit on the board)

- Consumers responded positively to the initiative.

In summer 1992, NYSE-Star appointed an Executive Director. Between spring and summer 1992, NYSEStar

- Increased the number of utilities participating in the program

- Trained and certified more builders 
- Established additional building requirements

- Developed promotional and marketing materials.

\section{Recent Developments}

NYSE-Star is expanding its network of certified builders through local builders associations. NYSE-Star developed and continues to offer builder training courses, and is developing promotional materials that can be used by builders to market the program. In addition, the organization plans to produce a Builder's Field Manual.

Between 1991 and spring 1993, approximately 900 New York builders were certified under the NYSE-Star program. Over 300 NYSE-Star homes have been constructed. Although the program has been extended throughout the state, the highest concentration of NYSE-Star-trained builders is in southern New York, Long Island, and the Albany area.

NYSE-Star's long-term goals include:

- Increasing the number of builders participating in the program

- Including gas and oil suppliers

- Becoming self-sufficient through builders' inspection fees

- Involving more manufacturers in the program

- Linking NYSE-Star homes with EEMs.

\section{Establishing a Linkage with EEMs}

In March 1993, Fannie Mae announced that NYSE-Star-certified homes qualify for EEMs. NYSE-Star has been promoting EEMS through articles and presentations at builder, realtor, and lender meetings, but has no record of EEMs being processed for NYSE-Star homes. NYSE-Star is aware that Exchange Mortgage Company on Long Island is actively offering EEMS; however, no clear linkage between NYSEStar homes and Exchange Mortgage has been forged.

\section{DOE Regional Office Housing Affordability Initiative Links HERS and EEMS}

In February 1993, the DOE New York Support Office began exploring the feasibility of two pilot projects-one in White Plains, NY, the other in Newark, New Jersey-linking energy efficiency with affordable housing. Under the proposed pilots, residences for low- to moderate-income families would be built on land donated by each municipality. The construction would be financed with bond issue money available for affordable housing, funds from HUD, the private sector, and, possibly, utility DSM funds. In addition, the State of New York Mortgage Association (Sony Mae) would be willing to guarantee a loan for the project. Four- to five-unit, multiple-family residences or two-family homes (totalling 60 to 75 units) would be constructed according to energy-efficiency specifications and would receive a HERS rating. The rental money collected would be used to amortize the loan.

The pilot is still at the preliminary stage. DOE-NYSO has met with the mayor of White Plains and the city's Director of Public Planning; both have endorsed the project and have suggested that a donation of municipal property could be arranged. A DOE-NYSO representative returned to White Plains in June 1993 to make a presentation to the City Council.

Negotiations with Newark are underway. The city is preparing to demolish and rebuild existing, dilapidated public housing. DOE-NYSO met with the mayor of Newark in mid-May about donating 
public housing land for the construction of low to moderate income energy-efficient housing. By mid-July 1993, DOE-NYSO expected to have completed the following:

- Meeting with the mayor of Newark, New Jersey, the Office of City Planning, and the Office of Public Housing to explain the pilot project linking HERS and EEMs (February - June 1993)

- Formally presenting the affordable housing proposal to the White Plains City Council, the mayor and the Director of Public Planning. If either of the Newark or White Plains sites should fall through DOE-NYSO will work to secure donations of land from other municipalities (February - June 1993)

- Developing a funding package for the project that would include bond issue money, funds from HUD, and support from the private sector once pilot locations have been confirmed. DOE-NYSO has held brief discussions with local lenders-Chemical Bank and the National Community Bank of White Plains-about underwriting an EEM for the project (February - June 1993)

- Convening a meeting of all potential players-the states, homeowners, individuals with low to moderate incomes, and utilities - to identify who is interested in participating in the project (mid-June - July 1993)

- Forming a New York-New Jersey Task Force comprised of 10 to 12 representatives from state and local government, lending institutions, and professional organizations from both states to provide feedback and help secure capital for the project. DOE-NYSO hopes to have a funding package in place by November 1993.

Once the construction sites and funding are in place, DOE-NYSO will solicit support from New York and New Jersey utilities (most likely focusing on Consolidated Edison of New York and Public Service Electric and Gas in New Jersey), and builders. In addition, DOE-NYSO will begin reviewing available HERS software.

DOE-NYSO plans to collect data on the pilot sites to prove the benefits of energy efficiency in low to moderate income housing. DOE-NYSO expects that data collection will include but will not be limited to consumption data collected through remote sensor monitoring and temperature drums. Other types of data will be defined by the information needs of lenders and those wishing to replicate the project elsewhere. DOE-NYSO plans to write a report describing the process and results of the project which can be disseminated to interested parties.

\section{Lending Institutions Known to Offer EEMs}

- Exchange Mortgage Company, Long Island

- The Bank of New York.

\section{Secondary Information}

\section{U.S. Department of Energy Report (1992)}

New York State Energy-Star administers the state's HERS program. The organization's Board of Directors includes representatives from the New York State Energy Office (NYSEO), the New York State Energy Research and Development Authority (NYSERDA), builders, and energy suppliers. The SEO is considering expanding the program to include ratings on existing homes (p. 28). 
NASEO Survey (1991)

New York is developing a HERS.

\section{Southern Electric International (Good Cents)(1992)}

Orange and Rockland Utilities, Inc. has a Good Cents program.

Barakat and Chamberlin, Inc. (1992)

The following utilities have DSM programs:

- Bath Electric, Gas and Water Systems

- Boonville Municipal Commission

- Brooklyn Union Gas Company

- Central Hudson Gas and Electric Corporation

- Consolidated Edison of New York, Inc.

- Long Island Lighting Company

- New York Power Authority

- New York State Electric and Gas Corporation

- Niagara Mohawk Power Corporation

- Orange and Rockland Utilities, Inc.

- Rochester Gas and Electric Company

- Sherrill Power and Light.

HERS Provider Information Network (Taylor 1993)

The following New York organizations are joining the HERS Provider Information Network:

- New York State Energy Office, Albany

- NYSE-Star, Albany.

Vories and George (1991)

The NYSEO, Residential Bureau, has developed a HERS which will be implemented by a non-profit corporation managed by the NY Home Builders Association. The program is being tested in cooperation with NY State Electric and Gas in their service territory in upstate NY. NYSEO:

- Is considering a certification system

- Is limiting eligibility to new single- and multifamily residences

- Has not yet determined if EEMs will be a part of the program (p. 96).

PERI (1992)

Steve Baden: New York is including HERS development efforts as part of its State Energy Plan (10/30/92).

FmHA (1993)

New York building codes/standards meet or exceed those of CABO MEC 89. 


\section{Other Sources}

Ely Reiss, President, New York State Home Builders Association, letter to DOE: NYSE-Star is a cooperative effort of industry, the utilities, the NYSEO and the NYSERDA. During 1992-1993 the organization plans to focus on the development of technical materials. A key component of the technical effort is the development of computer software for demonstrating compliance with the program's energy targets. NYSE-Star has reviewed existing software and has determined that ACRES [sic] software is best suited to the needs of the NY program. Particular advantages to the ACRES program are that it:

- Is based on a soon-to-be-promulgated national standard

- Possesses flexibility and can be adapted to the needs of NYSE-Star (9/24/92). 


\section{North Carolina}

Source of Information: David L. Smith, North Carolina Department of Commerce, Energy Division, 919/733-1893

\section{Background}

In 1991 and 1992, the North Carolina Department of Commerce, Energy Division, participated in the National HERS/EEMs Collaborative as a representative of the National Association of State Energy Officials (NASEO). The state plans a pilot program linking HERS with EEMs that is expected to begin in 1995.

\section{Recent Developments}

The North Carolina Energy Division convened an Advisory Committee in spring 1993 that represented a cross-section of interests in the state (e.g., home builders, lenders, real estate agents, appraisers, and energy suppliers). Plans are to form a public-private partnership. The Advisory Committee will advise the division as it develops guidelines for linking HERS with EEMs.

During 1993, the Energy Division will sponsor four focus group meetings in different parts of the state to let stakeholders express their concerns and input in the design of the pilot program. The Advisory. Committee will consider the comments generated, incorporating them as appropriate into its recommendations. NC Petroleum Violation Escrow (PVE) funds will support initial program costs.

Fannie Mae (FNMA) has expressed preliminary interest in the NC Pilot Program, although a formal agreement has yet to be devised. The Energy Division expects FNMA officials to be active in selecting lenders and in developing a system for recording EEM data. The Energy Division has approached several substantial lenders in the state, all of whom have indicated some interest in EEMs. The terms of their involvement are being negotiated.

\section{Lending Institutions Known to Offer EEMs}

Central Carolina Bank, Durham.

\section{Secondary Information}

\section{U.S. Department of Energy Report (1992)}

No response.

\section{NASEO Survey (1991)}

North Carolina is developing a HERS.

\section{Barakat and Chamberlin, Inc. (1992)}

The following utilities have DSM programs:

- Carolina Power and Light Company

- Duke Power Company

- Farmville Utility Department 
- Four County EMC

- High Point Electric Utilities Department

- Morganton Electric Utility

- North Carolina Electric Membership Corporation

- Virginia Power Company

- Washington Electric Utility

- Windsor Electric Department.

Utility Data Institute (1992)

Electricities of North Carolina, Inc. runs a DSM program.

\section{HERS Provider Information Network (Taylor 1993)}

The North Carolina Department of Economic and Community Development, Raleigh, is joining the HERS Provider Information Network.

\section{Vories and George (1991)}

1. The North Carolina Department of Economic and Community Development (state energy office), Energy Division, Residential Sector, is developing a HERS. The division:

- $\quad$ Plans to conduct a small pilot test as a first step

- $\quad$ Anticipates using a 0 -to-100 rating scale ("0" means energy inefficient)

- Will rate both new and existing construction

- Will be integrally linked with EEMs, particularly to finance energy improvements to existing homes (p. 98).

2. The North Carolina Alternative Energy Corporation is developing a voluntary, fuel-blind, newhome certification system for builders called the Exemplary Home Program. The corporation:

- Is testing technical aspects of the program

- Is researching the ERHA system and utility programs

- Will rate new, single-family residences

- Intends to link the program with EEMs (p. 97).

3. The Max Energy Efficient Home program, established by Duke Power in 1988:

- Assigns labels to homes meeting prescriptive standards

- Labels new, single- or multifamily electric homes

- $\quad$ Has labeled 7,500 homes

- Is not linked with EEMs; however, Duke Power is pursuing General Motors Acceptance Corporation (GMAC), Freddie Mac, and Fannie Mae acceptance of the Max Energy Efficient Home system (p. 63). 


\section{PERI (1992)}

Doug Culbreth: A group of approximately 30 participants has assembled in North Carolina to examine the HERS/EEM linkage issue. They are considering a pilot program. The North Carolina Department of Economic and Community Development (state energy office) is collaborating with utilities and industry to develop a HERS. The SEO is trying to initiate a pilot rating system $(6 / 12 / 92)$. 


\section{North Dakota}

No primary information available.

\section{Secondary Information}

\section{U.S. Department of Energy Report (1992)}

The State Energy Conservation Program is sponsoring a session on HERS at the statewide meeting of the North Dakota Home Builders Association. There is no program in place; nor is there pending legislation (p. 134).

\section{NASEO Survey (1991)}

North Dakota is interested in developing a HERS.

Barakat and Chamberlin, Inc. (1992)

The following utilities have DSM programs:

- Cass County Electric Cooperative, Inc.

- Grafton Light and Water Department

- Minnkota Power Cooperative, Inc.

- Montana-Dakota Utilities Company

- Mountrail-Williams Electric Cooperative

- Northern States Power Company

- Valley City Municipal Utilities. 


\section{Ohio}

Source of Information: Marcy Rood, Policy Development Supervisor, Ohio Department of Development, Office of Energy Efficiency, 614/466-6954; Jack Gayton, Deputy Director, Ohio Department of Commerce, 614/466-7050; and Dennis Tatum, Superintendent of Real Estate, Ohio Department of Commerce, 614/466-3411

\section{Background}

Since 1992, the Ohio Department of Development (ODOD), Office of Energy Efficiency (OEE) has been working with other Ohio agencies and organizations to develop and implement a voluntary, uniform Ohio HERS. At present, two utilities-Ohio Edison and Ohio Power-offer certification programs (Ohio Power's program is in the pilot stage).

In 1991, Governor Voinovich and the Ohio General Assembly directed the Public Utility Commission to develop a long-term energy strategy. In March 1992, the OEE, collaborating with the Ohio Department of Commerce (DOC) and the Public Utility Commission (PUC) sponsored a residential consumer meeting on finance and housing to explore the possibility of a uniform, voluntary Ohio HERS program linked with EEMs. The OEE planned to use the feedback from meeting participants to develop the HERS provisions for the OES. ${ }^{2}$ Those attending the meeting included representatives from lending institutions, real estate agencies, academia, state government, and the consumer's council.

The OEE convened a second meeting in November 1992 to further explore the feasibility of a uniform, voluntary statewide HERS linked with EEMs. Following a presentation by the Housing Resource Center (HRC; a Cleveland-based, non-profit housing laboratory) describing home energy ratings and existing HERS programs, the audience broke into five working groups representing utilities, state government, affordable housing, technical consultants, and builders/real estate agents. Each group discussed such issues as:

- Can there be more than one HERS tool?

- What information should be included on the HERS label?

- How can a HERS be accommodated to both new and existing homes?

- Would there be a statewide entity to provide oversight on the HERS program?

- Who should pay for the cost of rating houses?

- How can the rating system be of value to the resident, real estate agent, builder, developer, lender, utility, etc.?

At a plenary session, group leaders reported the results of their deliberations. According to OEE, the session generated much enthusiasm, especially among the utilities. Although lenders were invited to participate in the November 1992 meeting, representatives from the financial institution associations were unable to attend due to other commitments.

\footnotetext{
${ }^{2}$ The Office of Energy Efficiency is drafting the section of OES pertaining to HERS.
} 
The OEE was influenced by the National HERS/EEMs Collaborative's use of the consensus process and by the state advisory councils in Indiana and California. OEE recognizes that market penetration and the success of a voluntary program will depend on the involvement of diverse groups and their willingness to reach consensus.

\section{Recent Developments}

The five working groups ([1] builders, architects, engineers, home inspectors, and manufacturers; [2] state and federal government: [3] utilities; [4] lenders and real estate professionals; and [5] affordable housing) met January through March 1993 to discuss issues affecting their groups and to involve other interested parties and individuals. Issues included:

- Discussion and revision of a definition of an Ohio HERS drafted by OEE

- Developing a statement of purpose for each working group

- Outlining issues crucial to ensuring interest-group support for an Ohio HERS

- Designating members to a HERS statewide Steering Committee.

The DOC is assisting OEE to involve lenders in developing a linkage between HERS and EEMs in Ohio. Drawing upon its contacts in the real estate and lending communities, DOC and OEE convened a lenders/real estate working group in March 1993 to discuss lenders' and real estate agents' concerns about linking a HERS with EEMs and to designate representatives to the Steering Committee to be formed at the next OEE meeting. Meeting participants were receptive to EEMs and to the concept of a HERS: Lenders were most concerned about defining the standards that would be used in an Ohio HERS.

The OEE convened a third meeting on April 20, 1993. The agenda for that meeting included:

- Comments from the individual interest group meetings

- Presentations by representatives from the Alliance to Save Energy and the Center for Building Science and Research, Miami University

- Scheduling the development and implementation of a HERS program

- Introducing the 23 representatives from each working group who will serve on the statewide HERS/EEMs Steering Committee.

The mission of the HERS statewide Steering Committee is to serve as an advisory panel providing recommendations to the OEE for the development and adoption of a certified HERS that enables access to EEMs and other programs.

Most of these recommendations will be developed by four subcommittees comprised of some Steering Committee members and other representatives from various interest groups. These subcommittees are to present final recommendations for consideration by the HERS statewide Steering Committee by December 1993. The HERS Steering Committee will then present mutually agreed-to policy recommendations to OEE.

The four subcommittees and their objectives are as follows. 


\section{Technical Subcommittee}

- To develop minimum, uniform guidelines for certifying home energy and water efficiency rating tools and accrediting procedures

- To develop training curriculum and certification standards for raters.

\section{HERS/EEMs Linkage Subcommittee}

To work with lending institutions, realtors, appraisers, renovation contractors and developers to increase the use of energy-efficient mortgage programs at the time of purchase or refinancing.

\section{Marketing/Education Subcommittee}

- To formulate a marketing plan and strategy for implementing the HERS, including all targeted audiences

- To determine appropriate consumer education materials for HERS and EEMs

- To promote workshop opportunities for building inspectors, enforcement personnel, developers, and rehabilitators

- To design an effective method for display of the rating.

\section{Organizational Structure Subcommittee}

- To determine the most cost-effective structure for operating the HERS on a statewide basis and define those activities it is responsible for implementing

- To develop a budget for the program, potential contributors of a HERS organization, and linkages to public and private initiatives.

PVE funds are supporting Ohio's HERS/EEM activities. The current budget is $\$ 80,000$ for FY 1993 . By September 1994, OEE expects the first rating to be completed.

\section{Lending Institutions Known to Offer EEMs}

National City Bank, Northeast Branch, Akron.

\section{Secondary Information}

\section{U.S. Department of Energy Report (1992)}

The OEE is developing a HERS program that is linked with EEMs. In early 1993, OEE plans to convene a Steering Committee which will include, but will not be limited to, lenders, real estate professionals, and representatives from non-profit organizations. The Steering Committee will provide input and suggest ways to implement the linkage between HERS and EEMs in Ohio.

The Ohio State Strategy Interim Report included a recommendation for linking HERS with EEMs (p. 95). 


\section{NASEO Survey (1991)}

Ohio is interested in developing a HERS.

\section{Southern Electric International (Good Cents)(1992)}

The Ohio Edison Company has a Good Cents program.

Barakat and Chamberlin, Inc. (1992)

The following utilities have DSM programs:

- Centerior Energy Corporation

- Cincinnati Gas and Electric Company

- Columbus Southern Power Company

- East Ohio Gas

- Toledo Edison Company.

\section{Utility Data Institute (1992)}

The following Ohio utilities have DSM programs:

- American Electric Power

- Northern States Power Company.

HERS Provider Information Network (Taylor 1993)

The Ohio Department of Development, Columbus, is joining the HERS Provider Information Network.

\section{Other Sources}

Letter from Marcy Rood, Community Development Division, Office of Energy Efficiency to Barbara Farhar (NREL): Dayton Power and Light has a DSM program. 


\section{Oklahoma}

Source of Information: Brenda Lynn, Community Development Unit Manager, Oklahoma Department of Commerce, 405/843-9770

\section{Recent Developments}

Oklahoma does not have a HERS program or a mandatory state energy code. The state has no official state energy office. Many DOE programs are the responsibility of the Oklahoma Department of Commerce, such as the Weatherization Assistance Program (W AP), the Institutional Conservation Program (ICP), the State Energy Conservation Program (SECP), and the Energy Extension Service (EES). Conservation issues are not among the state's current priorities.

\section{Lending Institutions Known to Offer EEMs}

Bank of Oklahoma.

\section{Secondary Information}

\section{U.S. Department of Energy Report (1992)}

Does not have a statewide HERS program (p. 107).

\section{NASEO Survey (1991)}

Oklahoma is interested in developing a HERS.

\section{Southern Electric International (Good Cents)(1992)}

The following Oklahoma utilities have Good Cents programs:

- Duncan Power and Light

- Public Service Company of Oklahoma

- Stillwater Electric Utility.

Barakat and Chamberlin, Inc. (1992)

The following utilities have DSM programs:

- Alfalfa Electric Cooperative, Inc.

- Entex

- Oklahoma Electric Cooperative

- Oklahoma Gas and Electric Company

- People's Electric Cooperative

- Public Service Company of Oklahoma.

HERS Provider Information Network (Taylor 1993)

The Oklahoma Department of Commerce, Oklahoma City, is joining the HERS Provider Information Network. 


\section{Vories and George (1991)}

The Public Service Company of Oklahoma, which has administered a Good Cents program since 1984:

- Uses a computerized, menu-driven, performance rating with a point system

- Certifies new and existing, single- and multifamily electric homes

- Has certified 15,000 homes

- Has no direct link with EEMs; however, utility facilitates loans for existing homes if they meet Good Cents standards. Program is operated through the Bank of Oklahoma, which offers a five-year improvement loan of up to $\$ 10,000$ at $10 \%-11.45 \%$ interest. The utility backs the loan and obtains a lien on the home

- Offers cash incentive on heat pumps

- Has conducted studies comparing conventional buildings and Good Cents buildings to determine impact on peak demand and daily profile (p. 40). 


\section{Oregon}

Source of Information: Kathy Vega, Director, DOE Seattle Support Office, 206/553-1132; Jeff James, Director, Energy Technology Division, DOE Seattle Support Office, 206/553-2079; and Cindy Brown, Energy Analyst/Residential, Policy and Planning Division, Oregon Department of Energy, 503/378-8325

\section{Recent Developments}

The Oregon Department of Energy (ODOE) has been promoting EEMs with the housing industry since 1990 and is working to link EEMs with a uniform, statewide rating scale. Fannie Mae, Freddie Mac, and HUD/FHA accept the 1992 Oregon Energy Code for EEMs. However, ODOE is considering ways to tie Oregon's 1992 Energy Code, existing certification (Super Good Cents) and weatherization programs into a uniform rating scale.

In April 1993, the Alaska Housing Finance Corporation (AHFC) met with ODOE to outline a strategy for developing, promoting, and implementing a HERS linked with EEMs in Oregon. The Alaska/Oregon Home Energy Rating Work Plan is funded through the Pacific Northwest Regional Cooperative HERS/EEMs Collaborative pilot project and has three components:

1) Industry training: AHFC will provide ODOE with:

- A draft course outline and marketing plan for realtor and appraiser training on valuing and marketing energy-efficient homes

- A course outline for incorporating HERS and EEMs into FHA lender and appraiser training.

Course outlines and materials will be field tested by AHFC in 1993. ODOE plans to adapt the materials for use in Oregon and will encourage occupational licensing agencies to use the course outlines in their training programs for real estate agents and appraisers

2) Sharing information on HERS software development: AHFC is currently developing home energy rating and weatherization assessment software and will provide ODOE with a video presentation on the new software program. ODOE plans to approach Oregon's state weatherization program about cooperatively developing a HERS tool that also can be used for weatherization assessment

3) Bi-state industry cooperation: $\mathrm{AHFC}$ and ODOE will collaborate to include presentations on HERS and EEMs at the Oregon Real Estate, Home Builders, Appraisers, and Mortgage Bankers State Convention in 1993 and 1994 regional conferences of real estate professionals and builders.

Alaska will send drafts of its cooperative agreement with Oregon to Idaho and Washington SEOs.

The Pacific Northwest Regional Cooperative HERS/EEMs Collaborative is funded by DOE and meets quarterly to discuss issues pertaining to the development of a HERS. These include:

- $\quad$ Selecting a rating tool

- Involving the housing industry

- Recruiting and training raters

- Marketing the program

- Ensuring quality control

- Linking ratings with EEMs 
- Complying with the State Energy Code

- Linking a HERS with state weatherization programs.

The regional collaborative also provides a forum for the four states to share information on HERS and EEMs and to keep abreast of the developments within the National Home Energy Rating Systems Council.

\section{Lending Institutions Known to Offer EEMs}

- First Interstate Bank of Oregon, Portland, and Salem

- U.S. National Bank of Oregon, Salem

- West One Mortgage, Portland

- Norwest Mortgage, Portland

- First Security Bank, Salem

- Bank of the Cascades, Bend

- Liberty Federal Savings and Loan, Grants Pass, and Eugene

- Mortgage Management Controls, Salem

- Jackson County Federal Savings and Loan, Medford

- Washington Mutual, Salem, and Portland

- Key Bank of Oregon, Portland.

\section{Secondary Information}

\section{U.S. Department of Energy Report (1992)}

The only rating and certification program currently in place is the Super Good Cents program.

Oregon is promoting EEMs with builders, lenders, appraisers, manufactured housing dealers, real estate professionals, and home buyers. Fannie Mae and Freddie Mac have accepted the 1992 Oregon Energy Code.

Near term goals for Oregon's effort to link HERS and EEMs include:

- Convincing lenders statewide to accept the 1992 Oregon Energy Code as a basis to qualify home buyers applying for EEMs

- Collaborating with major industry players to design an Oregon HERS

- Coordinating Oregon's initiative to link HERS and EEMs with the NW Pacific Region's HERS/EEMs Demonstration program (p. 176).

NASEO SurveY (1991)

Oregon is developing a HERS.

\section{Southern Electric International (Good Cents)(1992)}

The following Oregon utilities have Good Cents programs:

- Bonneville Power Administration (BPA)

- BPA-Lower Columbia Area

- Canby Utility Board 
- Central Electric Cooperative

- Central Lincoln Public Utility District

- City of Ashland

- City of Bandon

- City of Cascade Locks

- City of Drain

- City of Forest Grove

- City of Monmouth

- Clatskanie Public Utility District

- Columbia Basin Electric Cooperative. Inc.

- Columbia Power Cooperative

- Columbia River Public Utility District

- Consumers Power, Inc.

- Coos-Curry Electric Cooperative

- Douglas Electric Cooperative

- Emerald Public Utility District

- Eugene Water and Electric Board

- Harney Electric Cooperative

- Hood River Electric Cooperative

- Lane Electric Cooperative

- McMinnville Water and Light

- Midstate Electric Cooperative

- Milton-Freewater Light and Power

- Northern Wasco County Public Utility District

- Oregon Trail Electric Consumers Cooperative

- Pacific Power and Light Company

- Portland General Electric Company

- Salem Electric

- Springfield Utility Board

- Tillamook Public Utility District

- Umatilla Electric Cooperative

- Wasco Electric Cooperative

- West Oregon Electric Cooperative.

\section{Bonneville Power Administration (Super Good Cents)(1993)}

The following Oregon utilities have Super Good Cents programs:

- City of Ashland

- City of Bandon

- Blachly-Lane County Cooperative

- Canby Utility Board

- City of Cascade Locks

- Central Electric Cooperative

- Central Lincoln Public Utility District

- Clatskanie Public Utility District

- Columbia Basin Electric Cooperative

- Columbia Power Cooperative

- Columbia River Public Utility District

- Consumers Power, Inc.

- Coos-Curry Electric Cooperative, Inc. 
- Douglas Electric Cooperative

- City of Drain

- Eugene Water and Electric Board

- City of Forest Grove Light and Power Department

- Harney Electric Cooperative

- Hood River Electric Cooperative

- Lane Electric Cooperative

- McMinnville Water and Light

- Midstate Electric Cooperative

- Milton-Freewater Light and Power

- City of Monmouth

- Northern Wasco County Public Utility District

- Oregon Trail Electric Consumers Cooperative

- Pacific Corporation

- Portland General Electric Company

- Salem Electric

- Springfield Utility Board

- Tillamook Public Utility District

- Umatilla Electric Cooperative

- Wasco Electric Cooperative.

Barakat and Chamberlin, Inc. (1992)

The following utilities have DSM programs:

- Ashland Department of Community Development

- Bonneville Power Administration

- Canby Electric Board

- Cascade Natural Gas Company

- Coos-Curry Electric Cooperative, Inc.

- Emerald People's Utility District

- Eugene Water and Electric Board

- Northwest Natural Gas Company

- Pacific Power and Light Company

- Portland General Electric Company

- Utah Power and Light Company.

HERS Provider Information Network (Taylor 1993)

The Oregon Department of Energy, Salem, is joining the HERS Provider Information Network.

Vories and George (1991)

1. In 1991, the Oregon Department of Energy (state energy office) began researching HERS programs:

- Anticipates developing a rating system based on the State Energy Code that links the energyefficiency value of a home to the energy code in effect when the home was built (code is updated every four years)

- Will be able to rate all residential structures (dwellings of three stories or less) 
- Allocated $\$ 50,000$ for program development, attempting to raise additional funds

- Will be linked with EEMs. A contractor is conducting field work to determine the feasibility of incorporating EEMs into a HERS program. Intend all buildings meeting Oregon's new energy code (effective January 1992) to qualify (p. 100).

2. The Conservation Division of the city of Ashland, OR has administered a Super Good Cents program since 1986:

- Certifies homes meeting certain prescriptive energy-efficiency standards

- Certifies new electric single- and multifamily homes; manufactured homes are also eligible

- Has certified 363 buildings (equals 550 residential units); approximately $98 \%$ of new electric homes are certified as Super Good Cents Homes

- Not directly linked with EEMs; however, BPA, through the State Energy Office, has hired a consultant to approach lenders about EEMs (p. 43).

PERI (1992)

Steve Baden: The Oregon State Energy Office is collaborating with utilities and industry to develop a HERS in Oregon (10/30/92).

FmHA (1993)

Oregon building codes/standards meet or exceed those of CABO MEC 89.

\section{Other Sources}

Javier Figueroa, Program Manager, Lender/EEMs, Washington State Energy Office, letter (and enclosures) to Barbara Farhar: Oregon participates in the Manufactured Homes Acquisition (MHAP) program (4/24/92). ${ }^{3}$

James Nybo, Conservation Analyst, Northwest Power Planning Council, letter (and enclosure) to Barbara Farhar: Oregon is participating in the NW Regional Energy-Efficient Mortgage Strategy $(5 / 6 / 92){ }^{4}$

\footnotetext{
${ }^{3}$ See Idaho for full description.

${ }^{4}$ See Idaho for full description.
} 


\section{Pennsylvania}

No primary information available.

\section{Secondary Information}

\section{U.S. Department of Energy Report (1992)}

In the 1980 s, the Pennsylvania Energy Office developed the "Home Energy Cost Estimator" program. The program helped homeowners evaluate home energy saving options (p. 50).

\section{NASEO Survey (1991)}

Pennsylvania has a HERS (Home ENERGY SCORECARD System) in a limited number of communities. The rating system is not linked with EEMs. State energy officials cited lack of industry support as a barrier.

\section{Southern Electric International (Good Cents)(1992)}

The following Pennsylvania utilities have Good Cents programs:

- Metropolitan Edison Company/GPU

- Pennsylvania Electric Company/GPU

- Pennsylvania Power Company.

Barakat and Chamberlin, Inc. (1992)

The following utilities have DSM programs:

- Allegheny Power System

- Duquesne Light Company

- Metropolitan Edison Company

- Pennsylvania Electric Company

- Pennsylvania Power and Light Company

- Pennsylvania Power Company

- People's Natural Gas

- Philadelphia Electric Company

- West Penn Power Company.

\section{HERS Provider Information Network (Taylor 1993)}

The following Pennsylvania organizations are joining the HERS Provider Network:

- Pennsylvania Energy Office, Harrisburg

- Grace Energy Center, Philadelphia.

Vories and George (1991)

1. The Pennsylvania Energy Office (PEO) Home Energy Cost Estimator program, developed in 1983, calculates the energy efficiency of a home in terms of cost. PEO is considering developing an alternative rating system since the program is no longer in use. The office is conducting research 
through the Southeastern Pennsylvania Energy Center in Philadelphia on the best approaches to using EEMs (p. 102).

2. The Excellence in Energy Efficiency, launched by the Philadelphia Electric Company in 1985:

- Is a pass/fail labeling program based on a home's compliance with certain prescriptive standards

- Certifies new, single-family and multifamily dwellings (up to three stories); emphasizes electric homes but will certify both gas and electric residences

- Labels 12,000 to 13,000 homes a year (approximately $25 \%$ to $30 \%$ of the new housing starts)

- Budgets approximately $\$ 500,000$ each year for program operation

- Promotes EEMs but does not know if they are used (p. 71). 


\section{Puerto Rico}

No primary information available.

Secondary Information

U.S. Department of Energy Report (1992)

The Department of Consumers Affairs (DOCA) is interested in HERS and EEMs (p. 69). 


\section{Rhode Island}

Source of Information: Vin Graziano, Rhode Islanders Saving Energy, 401/272-1040

\section{Recent Developments}

Rhode Islanders Saving Energy (RISE) conducts home energy ratings in Rhode Island. RISE announced a new initiative with the Rhode Island Housing and Mortgage Finance Corporation (RIHMFC) in March 1993. Under the new program, home buyers who buy a home designated by RISE as energy efficient will receive credit of up to $\$ 450$ against closing costs. Only first-time home buyers financing the purchase of their homes with a RIHMFC low-interest mortgage qualify for the credit. RISE will contribute onequarter of a percent of the mortgage amount to those who purchase a "Four-Star" energy rated home. For buyers of homes that do not qualify for the four-star designation, RISE will contribute an additional one-quarter of a percent if the buyer escrows the funds at closing to make the improvements necessary to earn the rating.

\section{Lending Institutions Known to Offer EEMs}

Rhode Islanders Home Mortgage Finance Corporation.

\section{Secondary Information}

\section{U.S. Department of Energy Report (1992)}

The Rhode Island Energy Office supported a HERS program administered by RISE and EEMs that were available through RIHMFC. Both the HERS programs and the RIHMC EEMs have been suspended ( $p$. 21).

Barakat and Chamberlin, Inc. (1992)

The following utilities have DSM programs:

- Blackstone Valley Electric Company

- Eastern Utilities Associates.

\section{HERS Provider Information Network (Taylor 1993)}

Rhode Islanders Saving Energy, Providence, is joining the HERS Provider Information Network.

\section{Vories and George (1991)}

1. RISE administers the RISE All Star Energy Rating Program:

- Adapted ERHA software

- Uses a Five-Star rating scale (translates into a 0-to-100 point scale)

- Has rated approximately 5,500 homes

- Promotes EEMs as part of program; no data available on number of EEMs

- Plans to expand rating capability to include multifamily buildings in late 1991 (p. 53). 
2. Rhode Island participates in the Boston-based Energy Crafted Homes of New England program (p. 57). ${ }^{1}$

\section{Hughes (1992)}

Rhode Islanders Saving Energy (RISE):

- Has rated over 7,000 homes and provided energy audits since $1978 ; 12 \%$ of the homes rated received Four Stars or more

- Has developed an energy rating checklist for audits of single-family, detached dwellings

- Suggests energy-efficiency improvements for homes receiving less than Four-Stars

- Awards a RISE ALL-STAR RATING seal to homes receiving Four Stars or better.

FmHA (1993)

Rhode Island building codes/standards meet or exceed those of CABO MEC 89.

\section{Other Sources}

Rhode Island Public Utilities Commission, information provided to Jan Eckert (NREL): The following Rhode Island utilities have residential DSM programs:

- Naragansett Electric Company

- Blackstone Valley Electric

- Newport Electric Company.

${ }^{1}$ See New Hampshire for full description. 


\section{South Carolina}

Source of Information: Jean Paul Gouffray, Program Information Coordinator, Governor's Office of Energy Programs, 803/734-0325

\section{Recent Developments}

South Carolina does not have a statewide HERS program. However, the state is considering developing a HERS linked with EEMs in the next five years.

\section{Secondary Information}

\section{U.S. Department of Energy Report (1992)}

Does not have a statewide HERS program (p. 71).

\section{NASEO Survey (1991)}

South Carolina energy officials are developing a HERS and are considering the Energy Rated Homes of America system.

\section{Southern Electric International (Good Cents)(1992)}

The following South Carolina utilities have Good Cents programs:

- Aiken Electric Cooperative

- Bamberg Board of Public Works

- Berkeley Electric Cooperative

- Black River Electric Cooperative

- Central Electric Power Cooperative

- City of Georgetown

- Coastal Electric Cooperative

- Edisto Electric Cooperative

- Fairfield Electric Cooperative

- Horry Electric Cooperative

- Lynches River Electric Cooperative

- Marlboro Electric Cooperative

- Mid-Carolina Electric Cooperative

- Palmetto Electric Cooperative, Inc.

- Pee Dee Electric Cooperative

- South Carolina Electric and Gas Company

- Santee Cooper

- Santee Electric Cooperative

- Tri-County Electric Cooperative.

Barakat and Chamberlin, Inc. (1992)

The following utilities have DSM programs:

- Carolina Power and Light Company

- Palmetto Electric Cooperative, Inc. 
- South Carolina Electric and Gas Company

- South Carolina Public Service Authority.

Utility Data Institute (1992)

Santee Cooper has a DSM program.

HERS Provider Information Network (Taylor 1993)

The South Carolina Governor's Energy Division, Columbia, is joining the HERS Provider Information Network.

FmHA (1993)

South Carolina building codes/standards meet or exceed those of CABO MEC 89. 


\section{South Dakota}

Source of Information: Marc McKillip, South Dakota Energy Office, 605/773-3603

South Dakota does not have a HERS program or an updated building code program. Energy officials in the South Dakota Energy Office (SDEO) are developing a state energy code to comply with EPACT requirements. By October 1993, SDEO hopes to make drafts of the code available for public review and comment.

SDEO is collaborating with the Sioux Falls Chapter of the Home Builders Association to develop a brochure entitled "Cost Affordable Standards for Homes" (CASH) suggesting energy conservation measures for consumers.

\section{Secondary Information}

\section{U.S. Department of Energy Report (1992)}

The South Dakota Home Builders Association and the Governor's Office of Energy Policy (GOEP) are sponsoring a Cost Affordable Standards for Homes program. Certification is granted for homes and buildings constructed to certain energy standards (p. 137).

\section{NASEO Survey (1991)}

South Dakota energy officials are developing a HERS.

Barakat and Chamberlin, Inc. (1992)

The following utilities have DSM programs:

- Beresford Municipal Power Department

- Black Hills Corporation

- Black Hills Power and Light Company

- East River Electric Power Cooperative

- Groton Electric Light and Power Department

- Midwest Gas

- Northern States Power Company

- Sioux Valley Empire Electric Association.

\section{HERS Provider Information Network (Taylor 1993)}

The South Dakota Governor's Office of Energy Policy, Pierre, is joining the HERS Provider Information Network.

\section{Vories and George (1991)}

The 1992 State Energy Conservation Plan contained provisions calling for the development of a HERS program and for the provision of technical assistance to builders (e.g., demonstration projects and HERS training). 
The South Dakota Governor's Office of Energy Policy (OEP; state energy office) is collaborating with the Home Builders Association to develop criteria for a HERS:

- Will implement a South Dakota HERS program in 1993

- May be linked with EEMs (p. 103). 


\section{Tennessee}

Source of Information: Jimmy Johnston, Program Manager, Department of Economic and Community Development, Energy Division, 615/741-2994

\section{Recent Developments}

Tennessee energy officials have no immediate plans for a HERS program. They are updating the state's building codes to meet CABO MEC 92 standards.

\section{Secondary Information}

\section{U.S. Department of Energy Report (1992)}

Does not have a statewide HERS program (p. 77).

NASEO Survey (1991)

Tennessee energy officials are developing a HERS.

Barakat and Chamberlin, Inc. (1992)

The following utilities have DSM programs:

- Memphis Light, Gas and Water Division

- Meriwether Lewis Electric Cooperative

- Oak Ridge Electric Department

- Tennessee Valley Authority.

Vories and George (1991)

The Tennessee Energy Division (state energy office) is working with Memphis Light, Gas and Power, and an engineering contractor (responsible for technical aspects) to develop a HERS:

- SEO will provide training services; utility will administer and market program

- Will rate existing, single-family homes (utility offers a new-home program to builders)

- Budgeted $\$ 50,000$ for HERS development

- EEMs not linked with program (p. 104). 


\section{Texas}

Source of Information: Wesley Leonard, Director, Energy Center, University of Texas at El Paso, 915/747-5494

\section{Recent Developments}

In 1990, the Energy Center (EC) at the University of Texas El Paso received funding from the Texas Governor's Energy Office to develop and pilot HERS software designed for use in arid climates. Through an arrangement with Energy Rated Homes of America (ERHA), the EC HERS Project modified ERH software to take into account evaporative air cooling and other features of homes located in arid climates. The EC trained raters and, between 1990 and 1993, 50 El Paso homes received ERH ratings.

The contract for the pilot program ends August 31, 1993. The EC has approached utilities and the home builders association about continuing the HERS project once state funding runs out, but neither party has expressed interest in administering the rating program.

\section{Secondary Information}

\section{U.S. Department of Energy Report (1992)}

No response (p. 112).

\section{Southern Electric International (Good Cents)(1992)}

The following Texas utilities have Good Cents programs:

- Bluebonnet Electric Cooperative

- Central Power and Light Company

- Central Texas Electric Cooperative

- City of Boerne

- City of Brenham

- City of Fredricksburg

- City of Giddings

- City of Lampasas

- City of Llano

- City of Lockhart

- City of Seguin

- City of College Station

- DeWitt Electric Cooperative

- Guadalupe Valley Electric Cooperative

- Gulf States Utilities Company

- Houston Lighting and Power Company

- Kerrville Public Utility Board

- Lower Colorado River Authority

- McCulloch Electric Cooperative

- New Braunfels Utilities

- Pedernales Electric Cooperative

- San Bernard Electric Cooperative

- San Marcos Electric Utility

- Texas-New Mexico Power Company. 
Barakat and Chamberlin, Inc. (1992)

The following utilities have DSM programs:

- Austin Electric Department

- Central Power and Light Company

- Cuero Electric Utility

- Denton Municipal Utilities

- El Paso Electric Company

- Entex

- Gulf States Utilities Company

- Houston Lighting and Power Company

- Kerrville Public Utility Board

- Lower Colorado River Authority

- New Braunfels Utilities

- San Antonio City Public Service Board

- San Marcos Electric Utility

- Seguin Electric System

- Texas Utilities Electric Company

- Texas-New Mexico Power Company

- West Texas Utilities Company.

\section{Utility Data Institute (1992)}

The following Texas utilities have DSM programs:

- Central and South West Services, Inc.

- City of Austin.

\section{HERS Provider Information Network (Taylor 1993)}

The Texas Government Energy Management Center, Austin, is joining the HERS Provider Information Network.

\section{Vories and George (1991)}

1. The Environmental and Conservation Services Department (city of Austin) has been rating homes through the Austin Energy Star Program since 1985:

- Uses Building Energy Thermal Analysis (BETA) software

- Rates homes according to a numerical score; must have minimum score of 100 (a One-Star rating) to receive Energy-Star label

- Labels new, single-family homes (ratings are fuel specific)

- Has rated over 2,000 homes; averages 600 per year

- Considering linkage with EEMs 
- Expanded program in Fall 1991 to include environmental issues; will be introduced as the "Green Builder" program (p. 59).

2. The University of Texas at Arlington began running the Texas Energy Extension Service in 1991.

- Developed alternatives to HUD standards which took into account the Texas climate

- Coordinates and promotes EEMs in Texas; meets with builders, lenders, and other building industry representatives. Has been contacting utilities that already have rating/certification systems (p. 106).

3. West Texas Utilities began the Energy Saving Plan Award program in 1983:

- Certifies homes meeting prescriptive list of standards for thermal envelope and mechanical equipment

- Certifies new and existing single-family and multifamily structures

- Has rated 9,000 homes

- Has contacted lenders about offering EEMs (p. 79).

\section{PERI (1992)}

Chris Roitch: Texas provides technical assistance to existing HERS programs through the Energy Extension Service. The focus is to integrate existing EEM programs, such as Fannie Mae's, with existing HERS programs (utility programs and the voluntary system used by the Texas Association of Home Builders). Another activity in Texas has been to work with the HUD Region Six office to develop an alternative to the 91103 documentation (Energy-Efficient Home Guideline) required for the HUD EEM. ${ }^{2}$ These Texas programs are limited to new homes (6/12/92).

\section{Hughes (1992)}

The Austin Energy Star Program:

- Has rated nearly 4,000 homes; completed 850 ratings during 1992

- Is debugging updated version of BETA software

- Is merging program into Green Builder program. Green Builder program rates four areas-water, energy, building materials, and solid waste-each of which is assigned a star.

${ }^{2}$ In 1988-1989, HUD changed its underwriting practice for qualifying borrowers to conform with procedures used by Fannie Mae and Freddie Mac. The department created Form 91103 which provided prescriptive criteria for energy efficiency. Builders in Region Six objected to these prescriptive measures and proposed a more flexible alternative. The new form, "Energy-Efficient Home Policy for HUD Region Six" is now in use. 


\section{Utah}

Source of Information: Rick Anderson, Director, Division of Energy, Utah Department of Natural Resources, 801/538-5428

\section{Recent Developments}

Utah energy officials are interested in HERS; however, no activity is expected at this time. In summer 1993, Utah state energy programs will be reorganized. Any activity pertaining to HERS and EEMs will be under the auspices of the state's Department of Community and Economic Development.

\section{Secondary Information}

OFTA Report (1992)

Does not have a statewide HERS program. The Utah banking community has resisted offering EEMs. Few if any of these mortgages have been made in the state, even though they have been available for years. Little interest has been shown in HERS (p. 139).

\section{NASEO Survey (1991)}

Utah energy officials are interested in developing a HERS.

Barakat and Chamberlin, Inc. (1992)

Provo City Power runs a DSM program.

\section{FmHA (1993)}

Utah building codes/standards meet or exceed those of CABO MEC 89.

\section{Other Sources}

Utah Public Utilities Commission, information provided to Jan Eckert (NREL): The following Utah utilities have residential DSM programs:

- Utah Power and Light Company

- Bridger Valley Electric Association, Inc.

- Dixie Escalante Rural Electric Association, Inc.

- Flowell Electric Association, Inc.

- Garkane Power Association, Inc.

- Moon Lake Electric Association, Inc.

- Mt. Wheeler Power, Inc. 


\section{Vermont}

Source of Information: Doug Lothrop, Director of Operations, Vermont Housing Finance Agency and Chairman of the Board of Energy Rated Homes of Vermont (ERH of VT), 802/864-5743, ext. 241; and Richard Faesy, President, ERH of VT, 802/865-3926

\section{Background}

Vermont has linked a HERS and EEMs since 1987. The effort to establish such a linkage began in 1986 when the Vermont Housing Finance Agency (VHFA) requested funds from the Vermont legislature to research, develop, and implement a link with EEMs. In 1986-87, the legislature allocated $\$ 500,000$ of Exxon oil overcharge funds for the program. An agreement between the Vermont Department of Public Service (DPS) and VHFA gave the VHFA administrative authority over the development of a link between a HERS program and EEMs. In 1987, the VHFA convened an EEMs Steering Committee-comprised of lenders, real estate agents, appraisers, utility personnel, fuel oil distributors, a homebuilder, a consumer advocate, and energy specialists-to advise the VHFA throughout the developmental phase of the program.

After researching several options, the VHFA in early 1987 decided on the Western Resources Institute's (WRI) Energy Rated Homes System. The HERS uses a five-star rating which includes recommendations for improvements that raise a home's rating to Four Stars. The VHFA contracted with WRI to develop HERS software appropriate to Vermont's climate, housing types, and energy costs. The VHFA convened a Technical Committee of energy professionals (architects, engineers, and home inspectors) to review the HERS software. The WRI system became the Energy Rated Homes of America program shortly thereafter.

In the fall of 1987, the VHFA incorporated Energy Rated Homes (ERH) of Vermont (VT) as a non-profit corporation. The corporation, under the management of VHFA and a Board of Directors, conducted home energy ratings, as well as trained and certified appraisers. In 1989, Vermont Energy Investment Corporation, under VHFA contract, assumed management responsibilities for ERH of VT.

In December 1988, the VHFA began a 12-month pilot program to test the link between the Vermont HERS program and EEMs. Three Vermont lenders-backed by Fannie Mae, Freddie Mac, and the VHFA - agreed to underwrite EEMs for the duration of the pilot. During the pilot, ERH of VT conducted 225 free home ratings in four counties. Of the homes rated during this initial pilot period, 63 resulted in EEMs. Fifty-one were stretched ratios on already energy-efficient homes.

In late 1988, the VHFA expanded the HERS program to include the rest of the state. It began charging a fee for ratings in 1990. To date, ERH of VT has rated more than 1500 homes. Since 1987, approximately 800 homeowners have used home ratings to negotiate EEMs. Between 1987 and 1989, out of 704 homes that were rated, 210 resulted in EEMs, of which 181 were stretched ratios on already energy-efficient homes.

Influenced by the work of the National Collaborative Consensus Committee during 1991, ERH of VT concluded that a pilot project was needed to generate data on default rates and market valuation of EEMs. In late 1991, ERH of VT convened a meeting with five Vermont mortgage companies and five bankers to outline a pilot program, its purpose, and the responsibilities of the lenders. The 10 lenders agreed to participate in the program.

Early in 1992, ERH of VT approached Fannie Mae with a proposal for a Vermont EEMs pilot project. Fannie Mae responded in April with an offer of $\$ 20$ million in secondary mortgage financing for EEMs in Vermont with VHFA acting on behalf of Vermont lenders. Under the agreement, Fannie Mae will back 
$\$ 20$ million in EEMs for approximately 250 loans. The agreement permits qualified borrowers or refinancers to include up to $\$ 5,000$ in energy-efficiency improvements in their mortgages with no additional appraisal or underwriting once the "base loan" is approved, provided that the value of the energy savings is documented as cost-effective by ERH of VT by using a modified Form 70A value calculation.

ERH of VT, with the support of Fannie Mae, VHFA, and the lenders, originally attempted to raise $\$ 20$ million in below-market rate investment capital from socially responsible investment firms and foundations to finance EEMs. This attempt was unsuccessful. In January 1993, ERH of VT decided to market EEMs at the standard interest rate and have Vermont lenders sell the mortgages directly to Fannie Mae instead of through the VHFA.

\section{Recent Developments}

Early in the spring of 1993, ERH of VT met with Fannie Mae and lenders to discuss marketing strategies and the recording of data. ERH of VT expects the pilot program to be market-driven and anticipates that lenders, builders, utilities, and real estate agents will promote the program. ERH of VT expects that an outside party (perhaps DOE or the Alliance to Save Energy) will be responsible for managing the data on EEMs. Although the details have not been worked out, Lothrop expects that the information to be collected will include the home's value, the cost of energy-efficiency improvements made, energy cost, the types of energy-efficiency improvements, the value of a home at sale, the default rate on EEMs, and the type of fuel used. ERH of VT anticipates that a control group on non-energy-efficient mortgages will be included. It is estimated that the tracking of EEMs will continue for a period of eight to ten years. ERH of VT expects the program to begin in September 1993.

\section{Lending Institutions Known to Offer EEMs}

- BancBoston/Bank of Vermont

- Banknorth Mortgage Company

- Chittenden Home Mortgage Company

- Marble Bank

- Merchants Bank

- National Bank of Middlebury

- Summit Financial Services

- Union Bank

- Vermont Energy Investment Corporation

- Vermont National Bank

- Vermont Federal Bank

- Vermont Housing Finance Agency.

HUD selected Vermont to be one of the five FHA EEM Pilot states.

\section{Secondary Information}

\section{U.S. Department of Energy Report (1992)}

Vermont links EEMs with a statewide, uniform HERS (p. 23). 
NASEO Survey (1991)

Vermont has a HERS (ERHA system) and has had a positive experience with the rating system. The rating system is linked with EEMs. Energy officials cited lack of recognition by appraisers as a barrier.

Barakat and Chamberlin, Inc. (1992)

The following utilities have DSM programs:

- Burlington Electric Department

- Green Mountain Power Corporation

- New England Electric System.

HERS Provider Information Network (Taylor 1993)

Energy Rated Homes of Vermont, Burlington, has joined the HERS Provider Information Network.

Vories and George (1991)

Energy Rated Homes of Vermont began administering the state's HERS program in 1987.

- Rates all types of homes (all fuel types)

- Uses $0-$ to- 100 rating scale; converts into a one- to five-star rating

- Has rated 1,100 homes

- Integrally linked with EEMs; approximately 500 rated homes have an EEM component. Since January 1990, 80\% of ratings involved EEMs (about 300 homes; p. 55).

PERI (1992)

Chip Coffay: The Vermont Pilot Project cleared the Fannie Mae regional approval process. It is funded for $\$ 20$ million for 250 homes mortgages. Historically, the Vermont Housing Finance Agency has worked with small banks and savings banks who sold mortgages to them. This relationship will be carried forward in the pilot program. These lending institutions will originate the loans and sell them to the Housing Authority which will in turn deal with Fannie Mae. Richard Faesy and Doug Lothrop are the project directors $(6 / 12 / 92)$.

Observer: Energy Rated Homes of Vermont performs an average of 250 ratings annually. Approximately $60 \%$ of these are used for EEMs, with the majority of those used to stretch the qualifying ratios on new homes. A few are used for existing homes and the balance are used by builders as a marketing tool. ERH of VT feels that further incentives are necessary to spur the existing home market. The direct cost (not including overhead) of performing a rating in Vermont is approximately $\$ 250$ (10/30/92).

\section{Hughes (1992)}

Energy Rated Homes of Vermont's HERS/EEMs program:

- Conducted 1,400 ratings (as of 10/92); approximately half used EEMs 
- Used $80 \%$ of lender-ordered ratings in EEMs (75\% used to stretch qualifying ratios [for buyers of Four-Star homes]; $5 \%$ used to escrow funds for upgrading energy-inefficient homes to Four Stars)

- Collaborates with Fannie Mae to develop EEMs pilot program

- ERH of VT ratings offered by five Vermont utilities (four electric; one gas) as part of newconstruction DSM programs.

\section{Other Sources}

Letter from Richard Faesy, President, Energy Rated Homes of Vermont, to Barbara Farhar (NREL): Sixteen Vermont utilities run DSM programs:

- Burlington Electric Light Department

- Central Vermont Public Service Corporation

- Citizens Utilities Company

- Green Mountain Power Corporation

- Hardwick Electric Department

- Hyde Park Electric Department

- Johnson Water and Light Department

- Ludlow Electric Light Department

- Lyndonville Electric Department

- Morrisville Water and Light Department

- Northfield Village Electric Department

- Stowe Electric Department

- Swanton Village Electric Light Department

- Vermont Marble Company

- Washington Electric Cooperative, Inc.

- Vermont Gas Systems, Inc. (8/6/93). 


\section{Virginia}

Source of Information: Christine Taylor, Executive Director, Energy Rated Homes of Virginia, Inc., 804/358-0892; Jennifer Snead, Energy Conservation Program Supervisor, Department of Mines, Minerals and Energy, Energy Division, 804/367-6883; and James A. Smith, Director, Energy Division, Department of Mines, Minerals and Energy, 804/367-6851

\section{Background}

In 1987-88, following the examples of states like Vermont and Alaska, the Virginia Department of Mines, Minerals, and Energy's Division of Energy (DE) convened a Technical Advisory Committee representative of builders, utilities, realty, and mortgage bankers to help define an energy-efficiency home rating program for Virginia and determine what software would best meet the program's needs. DE conducted an inhouse review of several existing HERS programs, including the California Home Energy-Efficiency Rating System (CHEERS), Good Cents, and Energy Rated Homes of America (ERHA). DE selected the ERHA system, which could be used to rate both new and existing homes, was fuel neutral and had been accepted for EEMs by Fannie Mae and Freddie Mac.

In 1989, DE contracted with ERHA for assistance in developing an Energy Rated Homes Program for Virginia. In cooperation with ERHA, DE established a task force consisting of builders, real estate professionals, HVAC, solar and weatherization contractors, utility representatives, and home inspectors. to help develop a HERS program for Virginia. A Steering Committee and a Technical Advisory Committee provided program oversight/direction and technical assistance, respectively. DE decided that a nonprofit corporation should be established to administer the program.

In January 1992, DE appointed a working Advisory Committee, consisting of a mortgage banker, a home builder, a real estate professional, electric and gas utility representatives, a solar industry representative, and home inspectors, to assist in establishing the nonprofit. The eleven member committee, organized into subcommittees, met monthly for six months and developed the documents necessary to form and implement the nonprofit corporation. Work of the committee included development of proposed bylaws, a proposed budget, and draft marketing and membership plans.

\section{Recent Developments}

In July 1992 ERH of Virginia was incorporated as a non-profit corporation, Energy Rated Homes of Virginia, (ERHV) Inc. The initial Board of Directors consisted of a mortgage banker, a home builder, a real estate professional, electric and gas utility representatives, a solar industry representative, a home inspector, and two-at-large members. The state allocated $\$ 300,000$ in PVE funds to support the operation of the nonprofit-\$150,000 each during fiscal years 1992 and 1993.

In February 1993, a parallel nonprofit trade organization-the Virginia Home Energy Rating Organization (VHERO) - was established to train and certify raters. VHERO will be self-supporting through rating and membership fees.

In March 1993, the DE launched a pilot test in Manassas, Virginia, to obtain data on 50 homes using ERHV, Inc. software. ERHV, Inc. expects the pilot to conclude in July 1993. The data obtained on the Manassas homes will be archived in ERHV, Inc.'s data base.

As of May 1993, VHERO has trained and certified 19 independent raters throughout the state. Sixty percent of the raters are affiliated with weatherization programs and serve homes in rural and mountainous areas. ERHV, Inc. and VHERO expect the independent contractors to market the rating program to 
consumers. In April 1993, the two organizations launched a marketing campaign to promote the linkage between HERS and EEMs, and the ERHV, Inc. program. Promotional activities include:

- Holding a press conference on the ERHV, Inc. rating program and its link with EEMs

- Sponsoring educational seminars for local boards of realtors, home builders associations, and lending institutions to introduce and explain the linkage between HERS and EEMs

- Securing a commitment from Fortune 500 companies based in Virginia (14 have company headquarters in Richmond, VA) to publicize ERHV, Inc. in their advertising and employee newsletters

- Developing an educational book for children explaining home energy efficiency and its effect on the environment (in the planning stage)

- Producing a video on home energy efficiency and the ERHV, Inc. program that can be loaned to consumers through video stores (in planning stage).

HUD selected Virginia to be one of five states participating in the FHA EEM Pilot Program. As of August 1993, at least 30 Virginia banks were prepared to offer EEMs.

VHERO is working with Virginia Electric Power Company (VEPCO) to increase the energy-efficiency standards of the utility's Energy Saver Home program. In 1994, VEPCO plans to expand its program to include an Energy Saver + Home. Homes with this designation would qualify for an ERHV, Inc. FiveStar rating.

\section{Lending Institutions Known to Offer EEMs}

- Nations Bank Mortgage Company, Richmond

- James Madison Mortgage Company, Fairfax

- Commerce Bank of Virginia, Richmond

- Heltzel Mortgage Corporation, Manassas

- Integra Mortgage Company, Charlottesville

- Tidewater First Financial Group, Inc., Virginia Beach

- Jefferson National Bank, Chesapeake and Grafton

- TransCoastal Mortgage Corporation, Newport News

- Life Mortgage Corporation, Suffolk

- Mid-Atlantic Financial Group, Inc., Richmond

- First Jefferson Mortgage Corporation, Norfolk

- Tidemark Mortgage, Newport News

- Ameribanc, Annandale 
- Sears Mortgage Corporation, Atlanta, Georgia

- Big Apple Mortgage Company, Inc., Winchester

- Union Financial Corporation, Richmond

- Carl I. Brown and Company, Portsmouth

- Commerce Bank, Virginia Beach.

\section{Secondary Information}

\section{U.S. Department of Energy Report (1992)}

On July 27,1992 , ERH of Virginia began administering Virginia's HERS program using an adaptation of ERHA software. Ratings will be conducted on both new and existing homes. The Virginia Division of Energy (state energy office) provides rater training and certification (p. 53).

\section{NASEO Survey}

Virginia has a HERS (ERHA system) and has had a positive experience with the rating system. The rating system is linked with EEMs.

\section{Barakat and Chamberlin, Inc. (1992)}

The following utilities have DSM programs:

- Rappahannock Electric Cooperative

- Virginia Power Company

- Washington Gas Light Company.

\section{Utility Data Institute (1992)}

Old Dominion Electric Cooperative runs a DSM program.

\section{HERS Provider Information Network (Taylor 1993)}

ERHV, Inc. Richmond, is joining the HERS Provider Information Network.

\section{Vories and George (1991)}

The Energy Saver Home Program, managed since 1983 by Virginia Electric and Power Company:

- Requires builders to meet a designated point score based on the total value of the features included in the home; buyers of these homes receive an home energy warranty from the utility

- Certifies new, single-family electric homes and new multifamily homes up to three-stories high

- Has certified 97,000 homes

- Promotes EEMs; no data on how many EEMs result from program (p. 77). 
Jim Smith: Energy Rated Homes of Virginia is incorporating to administer a HERS program in Virginia. The corporation should begin operating in January, 1993. ERH of VA is launching a pilot project and plans to rate at least 50 homes over the next year in Manassas, VA. The project will use the ERHA's uniform rating system and will, initially, rate only existing homes. New homes will be considered later $(6 / 12 / 92)$.

Observer: ERHV, Inc. is working with Virginia Power to redefine their Energy Savers program. ERHV, Inc. is also trying to unify the rating systems in the state. Other activities involve linking funding for lowincome housing with HERS, and producing a newsletter and public service announcements (10/30/92).

\section{Hughes (1992)}

Energy Rated Homes of Virginia began piloting a HERS program in 1992:

- The Virginia Energy Plan includes provisions for a HERS

- ERH of Virginia Board of Directors includes representatives of natural gas and electric utilities, municipalities, the home inspection industry, real estate sales and appraisal businesses, home builders, the solar power industry, and lending institutions

- Launched HERS pilot program in Manassas, VA in March 1992 (plans to rate 50 homes)

- Pilot includes educational programs on energy efficiency for lenders and real estate professionals, EEMs training sessions for lenders, and promotional and marketing campaigns to sell the concept to the public.

\section{FmHA (1993)}

Virginia building codes/standards meet or exceed those of CABO MEC 89.

\section{Other Sources}

Communication from Chris Taylor (ERHV, Inc.) to Jan Eckert (NREL): The following Virginia utilities also have DSM programs:

- Virginia Natural Gas (Energy-Efficient Home)

- Northern Virginia Electric Cooperative (Energy Saver Home)

- Appalachian Power

- Washington Gas (8/27/93). 


\section{Virgin Islands}

No primary information available.

\section{Secondary Information}

\section{U.S. Department of Energy Report (1992)}

The Virgin Islands Energy Office (VIEO) is exploring development of a HERS program. In lieu of a HERS, the Virgin Islands has created a point system for compliance with the revised Energy Conservation Code that achieves some of the HERS objectives (p. 80). 


\section{Washington}

Source of Information: Javier Figueroa, Program Manager, Lender/Energy-Efficient Mortgages, Washington State Energy Office, 206/956-2081

\section{Recent Developments}

In January 1993, the Washington State Energy Office (WSEO) developed an initiative to encourage state lenders to automatically implement EEMs when purchasers buy new, energy-efficient homes. Six banks-Sears Mortgage, Norwest Mortgage, Seattle Mortgage, U.S. Bank. Centennial Bank, and Washington Mutual Savings and Loan-have agreed to implement the EEM guidelines. Banks participating in the initiative will:

- Automatically apply EEMs when qualified borrowers purchase energy-efficient homes built to the Northwest Pacific Region's Model Conservation Standard (meets or exceeds CABO MEC '92)

- Market EEMs at workshops for bank employees and at seminars for real estate agents and others

- Track the number of EEMs underwritten as well as whether or not the ratio increase made a difference in the buyer's ability to qualify for the home loan. At present, only Sears Mortgage and Norwest Mortgage have tracking systems in place; Washington Mutual Savings and Loan and U.S. Bank are working out the logistics for collecting EEM data. In June 1993, the banks will release the first quarterly report on EEMs resulting from this initiative. Once all four banks have tracking systems in place, WSEO will formalize an agreement with Fannie Mae, Freddie Mac, and HUD to monitor the EEMs for default rates and for the resale values of foreclosed energy-efficient homes.

Norwest Mortgage, Sears Mortgage, U.S. Bank, and Washington Mutual Savings and Loan, all of which are multi-state mortgage companies, have agreed to offer automatic EEMs to qualified borrowers in Oregon, Montana, and Idaho. Norwest will extend the service to its customers in Alaska. WSEO hopes this initiative will serve as a model which could be replicated nationwide.

WSEO has developed an EEM addendum to the Washington Real Estate Sales Agreement which explains the benefits of EEMs and how they can be obtained. Pending approval by county boards of realtors and the Washington State Association of Realtors, the addendum will become a part of all residential real estate transactions in Washington. Five county boards are reviewing it. Three of the five counties represent a majority of the state's population. A major real estate firm, Prudential Preferred Properties in Kirkland, plans to use the addendum as soon as it has been approved. In the meantime, WSEO is modifying the addendum so it can be used as a fact sheet. Prudential plans to attach this sheet to the Real Estate Sales Agreement until the addendum is approved. Several other firms are interested in using the document, but are waiting until it has been endorsed by state and county real estate boards.

WSEO, in collaboration with Fannie Mae and 10 Washington public utilities, has designed a pilot program enabling home buyers qualifying under Fannie Mae's Community Home Buyers (CHB) Program to take advantage of utility-builder rebates when purchasing new, electrically heated homes in Washington. Banks approved by Fannie Mae to administer the CHB program will offer loans with flexible underwriting criteria to low- and moderate-income families. The program:

- Allows a higher debt-to-income ratio (up to $33 \%$ of a borrower's gross monthly income)

- Allows borrowers with no credit history to use non-traditional means to establish a good credit record 
- Requires a minimum down payment of five-percent, two-percent of which can be in the form of a grant or gift

- Waives the requirement that borrowers must have reserve funds to cover mortgage payments for two months.

Under the CHB pilot program, eligible borrowers purchasing new, electrically-heated homes may capture utility-incentive payments (normally paid to builders) to defray the cost of the down payment by up to two-percent; at least three-percent of the down payment must come from a borrower's own funds. Participating builders recruited by the utilities would set aside a percentage of new homes for sale to lowand moderate-income individuals while agreeing to pass the benefit of the utility rebate on to the buyers. Fannie Mae will monitor the loans issued through the CHB pilot program for signs of increased risk associated with utility-assisted financing. The pilot program is expected to begin in June 1993.

DOE's Seattle Regional Office, in collaboration with energy offices in Alaska, Oregon, Idaho and Washington, created a regional HERS/EEMs committee. Washington state does not have a HERS program; however, WSEO is fostering EEMs in the state and hopes that a HERS will be developed by the marketplace as demand for such a rating program increases. WSEO plans to convene a HERS/EEMs Regional Conference, which would include representatives from Fannie Mae, General Electric Mortgage Insurance Company, and the HUD regional office, as well as others from the real estate industry. The conference would provide a forum for explaining EEMs, introducing HERS, and describing the link between the two.

WSEO has contacted the Environmental Protection Agency's (EPA) Climate Change Grant program about developing and presenting a series of workshops on EEMs at EPA's regional offices.

In April 1993, Bonneville Power Administration (BPA) notified WSEO that funds supporting lender programs would be terminated as of July 1, 1993 due to budget cuts. The future of WSEO-initiated lender programs funded by BPA is in question.

\section{Lending Institutions Known to Offer EEMs}

- Sears Mortgage Corporation

- Norwest Mortgage

- Seattle Mortgage

- U.S. Bank

- Centennial Bank

- Washington Mutual Savings and Loan.

\section{Secondary Information}

\section{U.S. Department of Energy Report (1992)}

Washington state does not have a HERS. In lieu of a HERS program, Washington uses the State Energy Code (WSEC) and the Long Term Super Good Cents Program (sponsored by the Bonneville Power Administration) to assess the energy efficiency of homes.

In collaboration with BPA, the Northwest Power Planning Council, Fannie Mae, and Freddie Mac, the state administers an Energy-Efficient Mortgage Project. The Project encourages construction and purchase of new energy-efficient, electrically-heated homes by offering buyers special financing on their mortgages (p. 180). 
NASEO Survey (1991)

Washington has a HERS (utility-based [BPA] rating system). The rating system is linked with EEMs.

\section{Southern Electric International (Good Cents)(1992)}

The following Washington utilities have Good Cents programs:

- Alder Mutual Light Company

- Benton County Public Utility District No. 1

- Benton Rural Electric Association

- Big Bend Electric Cooperative

- Bonneville Power Administration (CBPA) Upper Columbia

- BPA-Puget Sound Area

- BPA-Snake River Area

- BPA-Upper Columbia Area

- Chelan County Public Utility District No. 1

- City of Centralia

- City of Coulee Dam Light Department

- City of Ellensburg

- City of Milton

- City of Port Angeles

- City of Sumas

- Clallam County Public Utility District

- Clark Public Utilities

- Columbia Rural Electric Association

- Cowlitz County Public Utility District

- Elmhurst Mutual Power and Light Company

- Ferry County Public Utility District

- Franklin County Public Utility District No. 1

- Grant County Public Utility District No. 1

- Grays Harbor County Public Utility District No. 1

- Inland Power and Light

- Kittatas County Public Utility District

- Klickitat County Public Utility District No. 1

- Lakeview Light and Power Company

- Lewis County Public Utility District No. 1

- Lincoln Electric Cooperative

- Mason County Public Utility District No. 1

- Mason County Public Utility District No. 3

- Nespelem Valley Electric Cooperative, Inc.

- Ohop Mutual Light Company

- Okanogan County Electric Cooperative, Inc.

- Okanogan Public Utility District No. 1

- Orcas Power and Light

- Pacific County Public Utility District No. 2

- Parkland Light and Water Company

- Peninsula Light Company

- Puget Sound Power and Light Company

- Seattle City Light

- Skamania County Public Utility District No. 1 
- Snohomish County Public Utility District No. 1

- Tacoma Public Utilities

- Tanner Electric Cooperative

- Town of Eatonville

- Town of Steilacoom

- Vera Water and Power

- Wahkiakum County Public Utility District.

\section{Bonneville Power Administration (Super Good Cents)(1992)}

The following Washington utilities have Super Good Cents programs:

- Alder Mutual Light Company

- Benton County Public Utility District No. 1

- Benton Rural Electric Association

- Big Bend Electric Cooperative

- City of Blaine Light Department

- City of Centralia

- Chelan County Public Utility District No. 1

- City of Cheney Light Department

- Clallam County Public Utility District

- Clark County Public Utility District

- City of Coulee Dam Light Department

- City of Ellensburg

- Elmhurst Mutual Power and Light

- Ferry County Public Utility District

- City of Fircrest

- Franklin County Public Utility District No. 1

- Grays Harbor County Public Utility District No. 1

- Inland Power and Light

- Kittitas County Public Utility District No. 1

- Klickitat County No. 1

- Lewis County Public Utility District No. 1

- Lincoln Electric Cooperative

- City of McCleary

- Mason County Public Utility District No. 1

- Mason County Public Utility District No. 3

- Nespelem Valley Electric Cooperative, Inc.

- Okanagan Company Electric Cooperative, Inc.

- Orcas Power and Light

- Parkland Light and Water Company

- Pend Oreille County Public Utility District No. 1

- City of Port Angeles

- City of Richland Energy Service Department

- Seattle City Light

- Seattle City Light North Service Center

- Skamania County Public Utility District No. 1

- Snohomish County Public Utility District No. 1

- Town of Steilacoom

- City of Sumas

- Tacoma Public Utilities Conservation Park 
- Tanner Electric Cooperative

- Vera Irrigation District No. 15

- Wahkiakum County Public Utility District

- Washington Water Power.

\section{Barakat and Chamberlin, Inc. (1992)}

The following utilities have DSM programs:

- Benton County Public Utility District

- Bonneville Power Administration

- Cascade Natural Gas Company

- Chelan County Public Utility District

- City of Richland

- Clark County Public Utility District

- Ellensburg Municipal Light Department

- Grant County Public Utility District

- Inland Power and Light Company

- Lewis County Public Utility District No. 1

- Northwest Natural Gas Company

- Port Angeles City Light

- Puget Sound Power and Light Company

- Seattle City Light

- Snohomish County Public Utility District

- Tacoma Public Utilities

- Washington Natural Gas.

\section{HERS Provider Information Network (Taylor 1993)}

The Washington State Energy Office, Olympia, is joining the HERS Provider Information Network.

\section{Vories and George (1991)}

1. The Washington State Energy Office (SEO) began promoting EEMs in 1988; SEO launched a statewide effort in 1991.

- Is working to create and maintain infrastructure for EEMs and increase their availability and demand

- $\quad$ Budgeted $\$ 90,000$ for 1992 (p. 107).

2. The Snohomish County Public Utility District Good Cents program, in operation since 1985:

- Certifies new homes using prescriptive standards, menu-driven performance software, or an energy budget calculation evaluating heat loss and heating system efficiency

- Has certified 6,152 homes

- Certifies new, electric, single- and multifamily homes, as well as manufactured homes

- Has promoted EEMs since 1985; however, less than ten EEMs have been completed 
- Dropped Super Good Cents program in July 1991; has adopted new Washington State Building Code which equals Super Good Cents standards (p. 45).

PERI (1992)

Steve Baden: Washington is involved in regional efforts with Alaska, Oregon, and Idaho to promote HERS and EEMs in the Pacific Northwest (10/30/92).

FmHA (1993)

Washington building codes/standards meet or exceed those of CABO MEC 89 .

\section{Other Sources}

Javier Figueroa, Program Manager, Lender/EEMs, Washington State Energy Office, letter (and enclosures) to Barbara Farhar: Washington participates in the Manufactured Homes Acquisition Program (MHAP)(4/24/92). ${ }^{3}$

James Nybo, Conservation Analyst, Northwest Power Planning Council, letter (and enclosure) to Barbara Farhar: Washington is participating in the northwest Regional Energy-Efficiency Mortgage Strategy $(5 / 6 / 92){ }^{4}$

\footnotetext{
${ }^{3}$ See Idaho for full description.

${ }^{4}$ See Idaho for full description.
} 


\section{West Virginia}

Source of Information: Jeff Herholdt, Manager, Fuel and Energy Office, West Virginia Development Office, $304 / 558-4010$

\section{Recent Developments}

West Virginia energy officials are interested in developing and implementing a statewide HERS, but are waiting until the HERS Council establishes national guidelines for home energy rating systems. The Appalachian Power Company-a subsidiary of American Electric Power in Columbus, Ohio-and a number of community action agencies have expressed interest in being HERS providers for West Virginia. Appalachian Power Company representatives met with the West Virginia Development Office to discuss implementing a HERS developed by the utility's parent company, American Electric Power.

\section{Secondary Information}

\section{U.S. Department of Energy Report (1992)}

Does not have a statewide HERS program (p. 57).

\section{NASEO SUrvey (1991)}

West Virginia is developing a HERS. Energy officials cited building industry opposition as a barrier.

Barakat and Chamberlin, Inc. (1992)

Washington Gas Light Company runs a DSM program.

HERS Provider Information Network (Taylor 1993)

The West Virginia Fuel and Energy Office, Charleston, is joining the HERS Provider Information Network.

\section{Other Sources}

Letter from Robert E. Wagner, Weatherization Coordinator, Eastern West Virginia Community Action Agency, Inc. to Barbara Farhar (NREL): The Eastern West Virginia Community Action Agency (EWVCAA), Inc. is applying for a local membership with Energy Rated Homes of America. EWVCAA has started to rate homes and plans to incorporate the HERS linked with EEMs into its housing and weatherization programs (7/9/93). 


\section{Wisconsin}

Source of Information: Mary Meunier, Wisconsin Energy Bureau, 608/266-2758; Scott Schmoller, Energy-Efficient Mortgage Coordinator, Wisconsin Energy Bureau, 608/267-2704; and Oscar Bloch, Program and Planning Analyst, Wisconsin Public Service Commission, 608/267-3588

\section{Background}

In July 1992, the Environmental Protection Agency's (EPA) Climate Change Grant Program awarded the Wisconsin Energy Bureau (WEB) a $\$ 100,000$ grant to develop and market a Wisconsin EEM program. The two-year grant will finance an EEM pilot program, to be initiated in the Madison area, and planning for statewide program implementation. An evaluation component to determine information regarding energy savings and environmental benefits also will be completed. When EPA awarded the grant, neither a HERS nor a standardized process for offering EEMs were in place.

\section{Developing a HERS}

In 1989 the Public Service Commission (PSC), in cooperation with Wisconsin's 10 Class A utilities, began development of a voluntary, uniform, statewide HERS. The rating system is now fully developed and is in the testing phase in order to calibrate the rating for statewide consistency. The PSC is coordinating the efforts of calibration of the HERS. Once the uniform HERS is complete it will be incorporated into the utility's auditing software. The PSC anticipates that the HERS software will be ready for use by summer 1993. Wisconsin's home heating fuel is predominantly natural gas; thus, it is likely that the gas utilities will actively use the HERS in new residential construction programs. It is expected in the future that utilities will provide the rating to customers of all existing homes.

To calibrate the home rating systems the PSC has created 12 to 15 computer models of homes with different energy requirements. These models will be rated by each utility using each utility's rating scale. The individual ratings will then be calibrated by the PSC to correspond to a one-to- 10 scale (one designates an extremely energy inefficient home; 10 indicates a home that uses no utility-supplied energy for space heating, cooling or water heating). Home energy use will be calculated on a Btu/ $\mathrm{ft}^{2} / \mathrm{HDD}$. Based on the rating system, it is estimated that a house with a rating of 7.5 to 8 or greater will be classified as an energy-efficient home and will be eligible for an EEM. Occupant behavior and lifestyle energy use (appliances and lighting) are not included in the home rating.

\section{Establishing a Linkage Between HERS and EEMs}

WEB is standardizing an EEM mechanism independently from the PSC's HERS program because of the delay in finalizing the HERS software. However, this mechanism will be able to easily incorporate the rated system once it is fully operational.

Discussion to implement the pilot program is currently underway with the shelter industries (including utilities, builders, real estate professionals, and lending institutions) in the Madison area. In addition, WEB is working with HUD, Fannie Mae, and Freddie Mac to establish specific energy-efficiency standards and formalize the EEM underwriting process for participating lending institutions. Once this information is obtained WEB will develop an EEM processing packet to be distributed to shelter industry participants.

An EEM Advisory Team will be formed to involve key shelter industry participants during the planning, development, and implementation of the pilot program. Participants in this public/private sector partnership will include representatives from state government, utilities, lending institutions, and the real 
estate and building industries. Team members will advise WEB on the development of the pilot project in addition to establishing means for continuing the program once the grant period ends.

\section{Secondary Information}

\section{U.S. Department of Energy Report (1992)}

The Wisconsin Public Service Commission (PSC) is coordinating the incorporation of Wisconsin utilities' home energy rating systems into a statewide rating system.

The Wisconsin Energy Bureau is implementing an Energy-Efficient Mortgage pilot program to promote the use and availability of energy-efficient mortgages in the state. The pilot will begin in fall 1992 and continue through spring 1994. It will focus on reaching real estate professionals, developers, mortgage lenders, and home buyers.

\section{NASEO SUrvey}

Wisconsin is developing a HERS. State energy officials are considering a utility-based rating system. They cited concern about the accuracy of the rating system as a barrier.

\section{Southern Electric International (Good Cents)(1992)}

Wisconsin Public Service Corporation has a Good Cents program.

Barakat and Chamberlin, Inc. (1992)

The following utilities have DSM programs:

- Dairyland Power Cooperative

- Madison Gas and Electric Company

- Manitowoc Public Utilities

- Northern States Power Company

- Wisconsin Electric Power Company

- Wisconsin Power and Light Company

- Wisconsin Public Power

- Wisconsin Public Service Corporation.

\section{Utility Data Institute (1992)}

Wisconsin Natural Gas Company has a DSM program.

\section{HERS Provider Information Network (Taylor 1993)}

The Wisconsin Department of Administration, Energy Bureau, Madison, is joining the HERS Provider Information Network.

\section{Vories and George (1991)}

The Wisconsin Public Service Commission is adapting a HERS tool that incorporates software used by Wisconsin's 10 Class A investor-owned utilities. The first utility planned to offer HERS ratings in January 1992: 
- System assesses space heating, cooling, and water heating, and produces a fuel-neutral rating

- Uses a one-to-10 scale (10 is maximum efficiency)

- Will rate new and existing single-family and multifamily homes of up to five units

- Budgeted between $\$ 65,000$ to $\$ 75,000$ to develop the HERS

- Will be linked with EEMs; logistics of linkage not yet worked out.

\section{Hughes (1992)}

The Wisconsin Energy Bureau received a two-year grant (from July 1992 through June 1994) from EPA to develop a statewide EEMs program:

- Will assemble an Advisory Council of representatives from the shelter industry to provide input on development of grant activities

- Will promote EEMs through seminars and workshops

- Will incorporate the HERS program being developed by the state's Public Service Commission (in final stage of development)

- Will evaluate EEMs pilot to determine benefits to the environment (i.e., a reduction in greenhouse gases).

FmHA (1992)

Wisconsin building codes/standards meet or exceed those of CABO MEC 89. 


\section{Wyoming}

No primary information available.

Secondary Information

\section{U.S. Department of Energy Report (1992)}

Does not have a statewide HERS program (p. 143).

\section{Southern Electric International (Good Cents)(1992)}

The following Wyoming utilities have Good Cents programs:

- Lower Valley Light and Power

- Utah Power and Light.

Bonneville Power Administration (Super Good Cents)(1993)

Lower Valley Power and Light has a Super Good Cents program.

Barakat and Chamberlin, Inc. (1992)

The following utilities have DSM programs:

- Black Hills Corporation

- Great Falls Gas Company.

HERS Provider Information Network (Taylor 1993)

The Wyoming Energy Conservation Department, Cheyenne, is joining the HERS Provider Information Network. 


\section{Appendix B}

Actions by Stakeholder Organizations Represented in the National Collaborative 


\section{Appendix B}

\section{Contents}

Introduction

Page

Consumer Groups

American Association of Retired Persons (AARP) $\ldots \ldots \ldots \ldots \ldots \ldots \ldots \ldots \ldots$

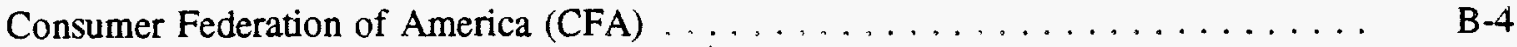

Home Builders

Energy Efficient Building Association (EEBA) $\ldots \ldots \ldots \ldots \ldots \ldots \ldots \ldots \ldots$

National Association of Home Builders (NAHB) $\ldots \ldots \ldots \ldots \ldots \ldots \ldots$

Home Energy Rating Industry

Energy Rated Homes of America (ERHA) $\ldots \ldots \ldots \ldots \ldots \ldots \ldots$ B-10

Southern Electric International (SEI), Good Cents Division . . . . . . . . . . B-10

Mortgage Lending Community

Department of Veterans Affairs (DVA) $\ldots \ldots \ldots \ldots \ldots \ldots \ldots \ldots \ldots \ldots$

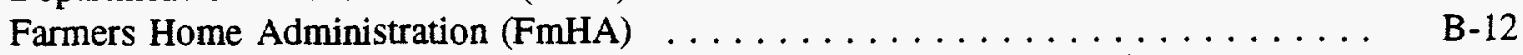

Federal Home Loan Mortgage Corporation (Freddie Mac) . . . . . . . . . . B-13

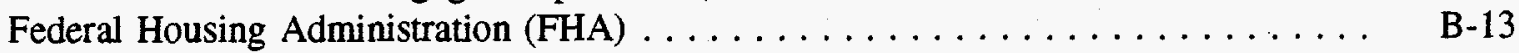

Federal National Mortgage Association (Fannie Mae) . . . . . . . . . . . B B-14

Mortgage Bankers Association (MBA) $\ldots \ldots \ldots \ldots \ldots \ldots \ldots \ldots \ldots \ldots \ldots$

Public Interest Groups

Alliance to Save Energy (ASE) . . . . . . . . . . . . . . . . . .

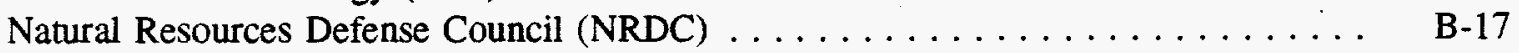

Real Estate Industry

American Society of Home Inspectors (ASHI) $\ldots \ldots \ldots \ldots \ldots \ldots \ldots \ldots$ B-18

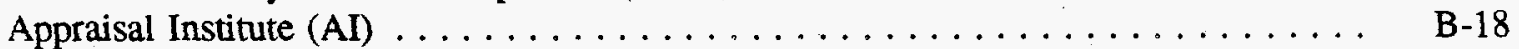

Federal Financial Institutions Examination Council (FFIEC),

Appraisal Subcommittee . . . . . . . . . . . $\ldots \ldots \ldots \ldots \ldots$ B-18

National Association of Energy-Efficient Mortgage

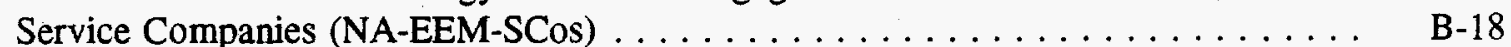

National Association of REALTORS (NAR) . . . . . . . . . . . . . . B-19

Remodelers

National Association of the Remodeling Industry (NARI) . . . . . . . . B-20

States

California Home Energy Efficiency Rating System, Inc. (CHEERS) . . . . . . . . B-21

National Association of State Energy Officials (NASEO) $\ldots \ldots \ldots \ldots \ldots \ldots \ldots$ B-21

State HERS Provider Information Network $\ldots \ldots \ldots \ldots \ldots \ldots \ldots \ldots$ B-21 


\section{Contents (Concluded)}

\section{Utilities}

Page

American Gas Association (AGA)

B-24

American Public Power Association (APPA)

B-24

Edison Electric Institute (EEI)

B-24 


\section{Introduction}

Appendix B assembles, by stakeholder group, primary information on the activities of the member organizations of the National Collaborative from the spring of 1992 to the spring of 1993 . Since the publication of $A$ Blueprint for Action, these organizations have undertaken a variety of activities to further the development of HERS and EEMs. Information on these activities was gathered from reports that members made during Collaborative Roundtables and also from personal interviews updating their activities. Each organization had the opportunity to review its entry in Appendix B. 


\section{Consumer Groups}

\section{American Association of Retired Persons (AARP)}

AARP's 1993 Public Policy Agenda takes a position on residential energy efficiency, including HERS and EEMs. The formal statement is:

Home energy rating systems (HERS) provide a baseline for measuring the energy efficiency of a home. When combined with energy efficient mortgages (EEMs), which permit a larger loan based on anticipated savings from reduced utility costs, HERS can assist homebuyers and promote energy-efficient new construction and rehabilitation. While HERS and EEMs have existed for some time, they have not been widely used and there are serious concerns about the reliability of rating systems, the adequacy of consumer protections, and the potential impact of ratings on home values. The National Energy Policy Act of 1992 directs the Department of Energy and HUD to develop voluntary home energy rating guidelines that can be used by state and local governments, lenders, utilities, secondary mortgage agencies, real estate agents, and builders. The guidelines are intended to enourage uniformity among rating systems and establish procedures for assuring the accuracy of the ratings. The law also established a technical assistance program to help states adopt and use rating systems, and a two-year energyefficient mortgage pilot program in five states.

The Association's position is: Any federal program designed to promote the use of HERS and EEMs should be voluntary. Homeowners should not be required to have their homes rated nor should standard (non-EEM) mortgage financing be conditioned on a home energy rating. DOE and HUD should incorporate professional standards for raters, scientifically valid measures, and full disclosure of consumer costs in any national guidelines for HERS/EEMs.

\section{Consumer Federation of America (CFA)}

- On May 29, 1992, CFA held a public forum to discuss the HERS/EEMs A Blueprint for Action. About 34 persons attended the forum to hear and ask questions of a panel of Collaborative representatives and John Millhone, Office of Building Technologies, DOE. Three points were emphasized during the comment period:

1. Most consumer leaders had never heard of EEMs.

2. Several consumer leaders said that experience shows that a nationwide HERS is unlikely to succeed unless targeted incentives such as tax credits, low interest loans, or EEMs are widely and readily available.

3. The program should be promoted not only as a way to achieve energy efficiency, but also as a way to make housing more affordable (it should include an inner city component).

CFA recommends the development and implementation of a public education program that picks up where the Collaborative's Awareness Technical Advisory Committee left off. The objective should be to make the country aware of the availability of EEMs and how to access EEMs. 
- $\quad$ Rebecca Cohen prepared an issue paper entitled "Addressing the Concerns of Real Estate Professionals about Their Role in Promoting Home Energy Rating Systems and Energy-Efficient Mortgages," January 1993, Regulatory Alternatives Development Corporation, Washington, D.C.

- $\quad$ CFA provided support to the Planning Committee of the HERS Council; CFA is represented on the Board of Directors (Rebecca Cohen). 


\section{Home Builders}

\section{Energy Efficient Building Association (EEBA)}

- $\quad$ EEBA's Criteria Committee is developing guidelines for constructing energy-efficient homes. The guidelines will take into account variations in building design, construction, and operation that differ according to climate region and specific construction markets. The Committee will consider how these three factors affect occupant comfort, health, and safety, building durability and maintenance, and building energy consumption.

The Criteria Committee is formulating the guidelines at three levels:

Level 1: Goals of an energy-efficient building

Level 2: Objectives of an energy-efficient building

Level 3: Criteria for an energy-efficient building.

These guidelines do not constitute a rating system.

As of April 1993, the EEBA Criteria Committee had completed Levels 1 and 2. The Level 3 portion of the guidelines will contain specific heat-loss criteria tailored to different climatic regimes; these are expected to exceed CABO-MEC 91 standards.

- $\quad$ EEBA is not a member of the HERS Council; however, the association follows HERS Council activities and continues to be interested in HERS and EEMs.

\section{National Association of Home Builders (NAHB)}

NAHB staff worked with Mineral Insulation Manufacturers Association and completed a simple compliance document for the CABO MEC; this will be offered to builders and others. This document could be used to show code compliance through a variety of means. If an energy-efficient mortgage can be granted on the basis of a home's meeting a model energy code, this document could be used to show code compliance. This could be useful in areas where there is no local code. Lenders could use it as certification to simplify their process.

- An article published in their newsletter on using EEMs to help sell homes received a favorable response.

- NAHB passed a resolution in September 1991 that urges public and private agencies to make energy-efficient mortgages more available. The resolution stated, in part:

... Be it resolved that the National Association of Home Builders urge public and private agencies that guarantee, insure or buy mortgages to establish policies and procedures that make it advantageous for builders to build, and buyers to buy, energy-efficient housing through more liberal underwriting criteria, increased mortgage amounts, and appraisal guidelines that recognize the financial advantages to the purchasers of homes with lower energy costs,

Be it further resolved that NAHB specifically support underwriting guidelines that stretch the qualifying ratios before, and in addition to, any 
consideration of compensating factors, for all homes that are built to NAHB's Thermal Performance Guidelines, or any standard that results in greater energy savings than NAHB's Thermal Performance Guidelines, and

Be it further resolved that NAHB specifically support underwriting guidelines that permit a buyer to apply energy savings directly to increased monthly mortgage payments by allowing a dollar for dollar increase in the monthly mortgage payment for each dollar saved in energy costs, when the house is compared to the average existing house in the area before, and in addition to, any consideration given to compensating factors.

- $\quad$ NAHB passed a resolution at its May 15, 1992, convention in Washington, D.C., that defined standards of affordability and cost-effectiveness in home purchasing. This definition supports NAHB's activities in building codes and standards, HERS, and EEMs. The definition states:

... NAHB defines increases in thermal performance standards to be "affordable" only if:

1. A buyer of a home who qualifies to purchase the home before the addition of the thermal performance standards would still qualify to purchase the same home after the additional cost of the energy-saving construction features

2. The buyer can include the cost of the energy-saving construction features in the mortgage

3. The buyer is not required to provide the incremental increase in the down payment due to the additional cost of the energy-saving construction features.

.. NAHB supports energy construction provisions that are cost-effective and affordable for the first-time home purchaser . . . NAHB has defined cost-effective to include the following:

1. Base all assumptions from the perspective of a typical first-time home buyer

2. Include benefits and costs over a time period that does not exceed the typical period of occupancy by the first-time buyer, which is approximately seven years

3. Evaluate the benefits and costs of successive increases in code requirements on an incremental basis, rather than comparing multiple aiternatives to a fixed-base case

4. Use a discount rate that accurately reflects the limited resources of first-time home buyers

5. Do not assume fuel price increases that exceed the assumed general rate of price inflation 
6. Assign a realistic residual value to the energy construction features at the end of the analysis period based on recognized appraisal methods for the energy-saving construction features.

The resolution called for builders to use these definitions in supporting NAHB positions on federal legislation, federal regulations, and model energy codes.

- $\quad$ NAHB also passed a resolution in May 1992 on "HUD Consideration of Energy-Efficient Mortgages." The resolution stated that, without a uniform national system in place, energyefficiency improvements were less affordable than if there were such a system. The resolution pointed out that existing EEMs are "fragmented, largely unknown by builders, lenders and the public, and therefore unused." The concern was expressed that many households will be disqualified for mortgages without uniform EEMs. EEMs were viewed as having a beneficial effect on home builders, home buyers, and the environment. In response to this situation, the resolution went on:

Now, therefore, be it resolved that the National Association of Home Builders requests the Department of Housing and Urban Development to direct HUD staff to move forward as quickly as possible to maintain the affordability of housing by implementing a Uniform Energy Efficient Mortgage that recognizes the value and the cost of energy efficiency improvements in the mortgage process while assuring that no family is disqualified for homeownership because of the imposition of energy efficient requirements.

- At its February 1993 convention NAHB presented an educational program on "Added Value through Energy Efficiency" that included discussion of energy-efficient construction practices and information on HERS and EEMs. The session was well attended and well received.

- Representatives of NAHB participated in the Planning Committee and Nominating Committee for the HERS Council during 1992 and 1993 and are currently involved in the Council's Board of Directors and Executive Committee. NAHB's interest is to be sure that HERS are tied to EEMs to keep the focus on the incentives for energy-efficient housing and to help ensure that EEMs are adopted and widely used.

- NAHB worked with the North American Insulation Manufacturers Association and Steven Winter Associates, Inc. to produce a simple compliance document for the CABO MEC entitled, Model Energy Codes Thermal Envelope Compliance Guide (copyrighted by North American Insulation Manufacturers Association in 1992) and distributed this guide to builders. HUD, CABO, and the National Conference of States on Building Codes and Standards (NCSBCS) were also involved in assembling the guide. Also, developers put together a videotape for local home builder associations (HBAs) on how to use the compliance guide.

- $\quad$ NAHB is beginning to develop training materials for builders on energy ratings and EEMs. The association will take responsibility for builder awareness. (NAHB believes that other organizations should also be responsible for increasing public awareness of the need for energy efficiency and EEMs as a means for getting more efficient housing.)

- An article was published in the NAHB newsletter in 1992 on using EEMs to help sell homes; this article received a favorable response. 
An NAHB staff member (William Sorrentino) served as a resource to the HUD Section 946 Task Force during 1992 and 1993. 


\section{Home Energy Rating Industry}

\section{Energy Rated Homes of America (ERHA)}

HERS are making increasing linkages in areas other than EEMs. For instance, the Arkansas ERHA is linking to the HUD Home Program. Model energy code compliance has been added to software in states with such codes. Other ongoing activities include making information available to the HUD Task Force, addressing the issue of duct leakage with testing and data base procedures, and looking at trainer certification needs, procedures, and issues.

- $\quad$ ERHA hosted a national conference, "Jump Starting National HERS and EEMs," attended by 100 people from the United States, Canada, and the United Kingdom. The conference was well attended and well received.

- ERHA established a committee to set standards and certification procedures for raters and rater trainers.

- They formalized an agreement for information exchange between ERHA and the Energy Foundation and the Foundation's HERS program in the United Kingdom.

- The ERH program in Arkansas has been linked with weatherization, rebates, community development block grants, the Job Training Partnership Act, utility assistance programs, and HOME-all with one set of standards and one application procedure called the Coordinated Home Improvement Plan. The ERH rating system will be used to train raters and improvement contractors and to provide quality control to the CDBG recipient programs.

- ERHA has conducted rater training for individuals in states that do not yet have home energy ratings sponsors, such as Michigan and Ohio.

- In Alaska, through the Alaska Housing Finance Agency, $6 \%$ of the mortgage is being loaned at no interest for five-star very efficient homes to cover the incremental cost of building to that standard. Alaska expects to process 5,000 ratings in 1993.

- ERHA has expanded rater training to include extensive blower door duct leakage, pressure balancing, and indoor air quality.

- REM/Rate 5 has now been added to the ERHA system. REM/Rate 5, developed by Architectural Energy Corporation in Boulder, Colorado, is a rating tool being employed in Colorado, Illinois, and Maine.

ERHA is a member of the HERS Council Board of Directors and participated in establishing the Council.

\section{Southern Electric International (SEI), Good Cents Division}

- The Sixth National Good Cents Conference was held in September 1992, with 240 attendees. Attendees have expressed considerable interest in the HERS Council. Good Cents has made presentations and has offered training activities to increase awareness of HERS/EEMs nationally. 
- In its activities to further HERS and EEMs during 1992 and early 1993, SEI has emphasized (1) participation in the National Collaborative and (2) participation in the Planning Committee to develop the HERS Council during 1992 and early 1993.

- The Good Cents program has begun to revise its products to prepare for the coming national program. For example, it is incorporating into its RBEP-2 software elements that will be consistent with the HERS verification and certification procedures that are likely to emerge from the HERS Council Technical Committee. The software will be more user friendly and will have increased flexibility to incorporate additional features. The program management expects the Good Cents software to pass any verification procedure, and also expects that the program itself will be certified.

- Good Cents plans to apply to the HERS Council to have representation from both Good Cents and Super Good Cents on the HERS Technical Committee.

- SEI has launched a cooperative advertising program with trade allies in energy efficiency in which the allies endorse Good Cents Homes. Ads will appear in the trade press, such as Builder magazine, to showcase to the builder audience a Good Cents home and inform them as to how such a home is advantageous to them. Some of the trade allies involved are Hurd Windows and Carrier Air Conditioning. Good Cents itself is not promoting the products of the trade allies providing the advertising endorsement of Good Cents Homes. The involvement and advertising endorsement of the trade allies is taken as industry recognition of the high market saturation and name recognition of the Good Cents program. SEI will be tracking the effect of the advertising program.

- SEI has organized several regional roundtable councils, notably in the Northeast, Southeast, and Texas, to bring together its client base-Good Cents utilities-to have them discuss common issues. Roundtables would promote cohesive promotion, information sharing about national trends, awareness that standards are changing, and sharing the same objectives. Nonmember utilities are also being invited to the roundtable councils.

- Through the roundtable councils, and in other settings, SEI is actively assisting utilities in linking their DSM programs with Good Cents. Several utilities are beginning to establish relationships with lenders so that they will be able to pursue EEMs when their Good Cents programs are certified as HERS. Depending on their public utility commissions, utilities can play significant roles in promoting and providing incentives for EEMs through the means identified in the Blueprint, such as indemnification of lenders or providing increments in down payments directly to lenders.

- The seventh annual Good Cents meeting is scheduled for September 4-6, 1993, in Houston. HERS/EEMs will be discussed. 


\section{Mortgage Lending Community}

\section{Department of Veterans Affairs (DVA)}

- DVA participated on the HUD Section 946 Interagency Task Force for Energy-Efficient Housing and has been represented at several meetings of that group chaired jointly by HUD and DOE.

- Public Law 102-547, enacted October 28, 1992, requires DVA to demonstrate the feasibility of EEMs. The most important provision of P.L. 102-547 from the HERS/EEMs perspective is that the cost of energy-efficiency improvements up to $\$ 6,000$ can now be added to a VA mortgage for an existing home without any value determination. For improvements costing between $\$ 3,000$ and $\$ 6,000$, there must be a determination that the improvements are cost-effective. "Cost-effective" is defined as meaning that the monthly increase in the mortgage payment is offset by the savings in monthly energy costs. No such documentation is required for improvements costing up to $\$ 3,000$.

P.L. 102-547 includes active and passive solar heating and cooling systems in its definition of allowable energy-efficiency improvements.

P.L. 102-547 establishes a new class of veteran, a Reservist, that will create more demand for loans. Other new programs will provide more flexibility to lenders and borrowers, including the availability of a new adjustable-rate mortgage (ARM) and allowing veterans to negotiate an interest rate. Another new program establishes a direct loan program for Native Americans who buy or build on trust land.

- DVA is revising all existing pamphlets for home buyers to give greater emphasis to the availability of EEMs. DVA is also revising its manual and other instructional material for participating lenders. In addition, DVA is publicizing the availability of EEMs through public speaking.

- In May 1993, the DVA will have preliminary figures on the number of EEMs underwritten by the DVA since October 1992.

\section{Farmers Home Administration (FmHA)}

FmHA will continue to enforce its thermal performance standards in its rural housing programs. The agency is in the process of revising its requirements to recognize other energy codes and standards that are comparable to the agency's. The agency will also continue to recognize the improved ability of its borrowers to repay their loans when they purchase energy-efficient housing.

FmHA has participated in the HUD Section 946 Task Force to develop a uniform plan for energy-efficient mortgages.

As reported in the section on legislation, Section 101 of the Energy Policy Act of 1992 requires FmHA to require codes or standards for all of its housing programs in all states that are equal to or more stringent than CABO-MEC 89. (States that currently meet this requirement are listed in Appendix A.) In practice, this requirement is comparable to what FmHA already does.

FmHA does not offer EEMs because it determines borrowers' repayments with a budget rather than a ratio, resulting in repayment being a direct function of energy savings. 


\section{Federal Home Loan Mortgage Corporation (Freddie Mac)}

- In an attempt to understand existing data on the performance of energy-efficient mortgages, Freddie Mac undertook an analysis using a list of 141 Energy Rated Homes of Vermont customers who had received a home energy rating and subsequently utilized an EEM. Analysts found that Freddie Mac had purchased 41 out of the 141 mortgages. This small sample did not permit them to arrive at conclusions concerning the default rates for EEMs as compared with default rates for other mortgages.

- Energy Rated Homes of America sent Freddie Mac a list of 8,000 homes in Vermont, Rhode Island, Arkansas, and Alaska that had been rated. Freddie Mac analysts validated a preliminary list of approximately 250 homes where Freddie Mac may have purchased the mortgage. However, no conclusive results could be reported because of small sample sizes.

- Freddie Mac participated in the HUD Task Force, and is currently waiting for the Task Force recommendations to be released before engaging in any revisions to its EEMs program.

- $\quad$ Although Freddie Mac views Fannie Mae's participation in the HERS Council as representing its interest, it will discuss whether to join the council.

- In a February 20, 1993, article by Leland Brendsel, chairman and CEO of Freddie Mac, in Nation's Building News, Freddie Mac's EEM program was described. The article said that the program allows borrowers to use actual energy savings in qualifying for their mortgages. The article gave an example of a loan in which a borrower could qualify for a $31 \%$ ratio and a higher mortgage with an energy-efficient home that provided utility bill savings of $\$ 90$ a month. The article urged readers to help Freddie Mac to make the program work by providing "ideas on ... ways we can make the program better for buyers, builder, realtor, and lender."

- Freddie Mac staff has noted that, whenever an article has appeared in the newspapers about its EEMs program, numerous telephone inquiries are made, virtually none of them from consumers. Instead, small entrepreneurs looking for a business opportunity make the inquiries.

\section{Federal Housing Administration (FHA)}

- $\quad$ FHA (Morris "Bud" Carter) is directing the HUD Task Force to develop a plan for uniformity among energy-efficient mortgage programs under the Affordable Housing Act. Its goals are to (1) focus on issues in the Blueprint and any others identified to make EEMs more friendly, (2) to remove barriers to EEMs, (3) to identify data needs for agencies, and (4) to prepare for pilot programs in which agencies can participate.

- Under the Energy Policy Act of 1992, FHA is also responsible for selecting five states to serve as pilot tests for EEMs concepts.

- FHA conducted training for its staff on EEMs concepts in March 1993, and will train lenders and others in the five pilot states from June through August 1993. 


\section{Federal National Mortgage Association (Fannie Mae)}

- Fannie Mae (Chip Coffay) is serving as a member of the HERS Council Board of Directors.

- Fannie Mae is working with partners in the State of Washington to develop a program in which local utilities, in cooperation with the Bonneville Power Authority (BPA), are providing $\$ 2,900$ in rebates to builders of efficient new electric homes. The state will provide $\$ 900$ of the rebate and BPA $\$ 1,500$ to $\$ 2,500$. The builders have the option of using the rebate to reduce the price to the buyer.

- Also, Fannie Mae is allowing buyers to make a down payment of 3\% (instead of the usual $5 \%$ required) for these efficient homes. This " $3 / 2$ option" allows the borrower to use third-party sources, such as a utility DSM program, for the other $2 \%$ of their down payment. This proviso helps builders by increasing the pool of potential home buyers by $5 \%$ to $10 \%$.

The Washington State Housing Finance Commission is considering using federal tax-exempt bond issue proceeds in support of this program. Bonds will be used to reduce the interest rate for firsttime home buyers below $115 \%$ of median income by $1 \%$ to $1-1 / 2 \%$. However, the program is currently on hold because the authority to issue federal tax-exempt bonds expired June 30, 1992. Federal legislation, needed to extend this authority, is expected in 1993.

Fannie Mae is also involved in a pilot program with the Vermont Housing Finance Authority (VHFA) to provide $\$ 20$ million in financing for 250 to 300 energy-efficient homes. The program allows inclusion of cost-effective improvements up to $\$ 5,000$ with no reappraisal and no additional underwriting. The VHFA will collect and report data on the mortgages in the pilot program.

Fannie Mae has developed a pilot program with a Maryland electric utility-the Southern Maryland Electric Cooperative-that is similar to the Washington State program, using a rebate to builders of efficient new electrically heated homes, paid by the utility. Home buyers will use the rebate as a source of down payment and closing cost assistance.

- Fannie Mae has also had preliminary discussions with North Carolina energy officials concerning a potential EEMs pilot program in that state.

- Fannie Mae has participated in the HUD Task Force that is developing a plan for a uniform EEMs program.

- Fannie Mae, through the Community Home Buyers program, permits lenders to be more flexible in underwriting to assist lower- to moderate-income home buyers by allowing higher debt-toincome ratios in mortgage financing.

- In data analysis, Fannie Mae received a list of 10,000 homes from ERHA that had received ratings. Of these, 1,200 were found in the Fannie Mae data base; 200 of these loans had been paid off. Approximately $60 \%$ of the remaining 1,000 were 30 -year fixed mortgages, $15 \%$ were 15-year fixed mortgages, $12 \%$ were ARMs, and the remainder were biweekly, growing equity, and second mortgages. Of the 1,000 mortgages still held by Fannie Mae, 50\% had loan-to-value (LTV) ratios of less than $70 \%$, and $13 \%$ had LTV ratios of $70 \%$ to $75 \%$. This distribution is typical of the majority of loans in Fannie Mae's portfolio. Because of these LTV ratios, the majority of those loans would not have faced difficulty in qualifying for Fannie Mae's EEM program. But, Fannie Mae was unable to reach conclusions about the default rate performance of the mortgages on the rated homes. 


\section{Mortgage Bankers Association (MBA)}

- $\quad$ MBA is trying to raise the visibility of EEMs. They have published a synopsis article in Statelines, which is mailed to all of their members. They also published an article in the newspaper Real Estate Finance Today to let lenders know what is going on and that other lenders are involved in providing EEMs to borrowers.

- $\quad$ MBA is following with interest the proposed increase in energy taxes, as well as the HUD pilot program that may lead to increased consumer demand for EEMs. The association continues to publish occasional articles on EEMs in Real Estate Finance Today.

MBA has designated Bruce Bowler, CEO, Universal Lending Corporation, Denver, CO, to represent the organization on the HERS Council Board of Directors. The HERS Council Board approved the nomination of Mr. Bowler and seated him on the board. 


\section{Public Interest Groups}

\section{Alliance to Save Energy (ASE)}

\section{- $\quad$ EEMS Activities}

- ASE staff members (Malcolm Verdict, Bion Howard) participated as technical resources to the HUD Section 946 Task Force on the development of a uniform plan for EEMs. Staff members collected and presented technical issue background papers for use by task force members and presented options for task force consideration.

- ASE participated in the ERHA conference on "Jump Starting EEM/HERS" in Little Rock, AR, in February 1993.

- $\quad$ HERS Council Formation Activities (Malcolm Verdict)

- Charter member and member of Planning Committee, including numerous meetings and conference calls

- Chaired Bylaws and Membership committees

- Served on the Nominating and Technical Fast Track committees

- Elected Vice-Chair of the Board of Directors and to the Executive Committee

- Co-chaired March 1993 organization meeting and reported on bylaws to the membership.

- National HERS/EEMs Collaborative Participation

- Staff participated in Collaborative Consensus Committee meetings in 1991 and 1992.

- ASE staff co-chaired (Bill Prindle) and served as member (Bion Howard) of HERS Technical Advisory Committee and presented technical issue papers.

- Malcolm Verdict participated in the subgroup on the future of the Collaborative.

- ASE presented detailed comments on Blueprint review draft.

HERS/EEMs Outreach and Education Activities

- Staff served on the California CHEERS Mortgage Finance Committee in 1992.

- Staff responded to 200 to 300 written requests for information on EEMs from a September 1992 Home Energy magazine article.

- Staff answered numerous phone inquiries from the states of VT, OH, CA, VA, WV, and MD concerning HERS/EEMs and the general public.

- Staff conducted a HERS/EEMs presentation at the Appalachian Weatherization Conference, Davis, WV, January 1993. 
- Staff conducted a workshop on HERS/EEMs at the March Energy-Efficient Buildings Conference in Boston, MA, March 1993.

- Staff conducted a session on legislative requirements of EPAct 1992 legislation for EEMs/HERS at the Affordable Comfort Conference in Philadelphia, PA, March 1993.

Other Activities

- ASE is developing a pilot program to provide a targeted technical and implementation information package to primary lenders, real estate professionals, and appraisers.

- ASE is providing expert advice to selected Hill staffers and elected officials on incorporation of technical and policy clarifications on HERS and EEMs programs for consideration in omnibus federal energy legislation.

- ASE staff members are working with NREL on the potential development of standard practices useful in the verification of HERS tool efficacy and functionality.

- The alliance worked with the EEBA to help plan their 11th conference, held March 1993. A major residential energy-efficiency marketing track is planned to educate utilities, building officials, lenders, and builders on HERS and EEMs, including evolving Green Building Rating concepts.

\section{Natural Resources Defense Council (NRDC)}

- NRDC was active in advocacy efforts in Congress for the inclusion of an EEMs pilot program in the Energy Policy Act of 1992.

- NRDC was one of the recipients in the Jones Foundation grant to prepare a package for the Clinton administration urging it to become more involved in a national commitment to EEMs beyond the energy bill and the Blueprint. NRDC held a meeting with participants in the project and worked on analysis of the environmental benefits of a program in which 1 million EEMs were achieved in 1996.

- An NRDC staff member (Dan Lashof) testified in March 1993 before the U.S. House of Representatives Ways and Means Committee on tax ideas on efficiency and renewables. Preferential tax treatment on the energy-efficiency portion of an energy-efficient mortgage would be an additional incentive to use EEMs.

- Marika Tatsutani of the NRDC staff presented on collaborative processes at the Energy Daily Conference in September 1992 in Washington, D.C., and made reference to the National Collaborative. 


\section{Real Estate Industry}

\section{American Society of Home Inspectors (ASHI)}

ASHI believes that home inspectors have a significant role to play in rating the energy efficiency of housing. Home inspectors reach approximately $50 \%$ to $75 \%$ of homes on the real estate market. ASHI estimates that $30 \%$ of these would benefit from an energy-efficient mortgage. For example, a typical situation in which an EEM would be appropriate is a 20-year-old house for which the client has no funds to do repairs. The borrower could obtain the cash to repair and improve the home through an EEM.

ASHI is concerned that the HERS industry provide information that the lending community will accept. ASHI plans to join the HERS Council. On the technical side, ASHI would like to give input to the rating and the manner in which ratings are conducted.

ASHI is investigating the performance and cost-effectiveness of rating software currently on the market. They are interested in software that rates, audits, and provides detailed information in a clear, easy-to-read format. The association believes that the software used for HERS should be simple to use and understand. ASHI believes that EEDO software, produced by the Bart Hill Association in Pennsylvania, is one of many available leading software packages. ASHI believes that it is accurate, easy to use, and relatively inexpensive, at a price of $\$ 395.00$. The Association of Energy Engineers has publications that list several: available software systems for home energy ratings.

ASHI is assembling a task force to develop a directory of HERS software for its membership. ASHI works closely with real estate professionals.

\section{Appraisal Institute (AI)}

- AI is a member of the HERS Council. Donald E. Kelly, Vice President for Washington Operations, represents the organization on the HERS Council Board of Directors.

- AI will publish an article on the March 1993 HERS Council meeting in its monthly newsletter, Appraiser News. AI will continue to use Appraiser News as a forum for educating AI members about HERS/EEMs.

- AI may include discussion of HERS/EEMs at their July 1993 Annual Conference in Reno, NV.

\section{Federal Financial Institutions Examination Council (FFIEC), Appraisal Subcommittee}

Ted Baker plans to be involved in the HERS Council; however, neither FFIEC nor the Appraisal Subcommittee has a role in HERS/EEMs. The FFIEC's role is to monitor the implementation of Title XI of Federal Institutions Reform Recovery and Enforcement Act and the licensing by the states of real estate appraisers. Mr. Baker was a member of the National Collaborative and plans to continue his involvement with HERS/EEMs out of individual interest.

\section{National Association of Energy-Efficient Mortgage Service Companies (NA-EEM-SCos)}

- The organization obtained a grant from the Alton B. Jones Foundation, along with the Natural Resources Defense Council and others, to prepare a letter to President Clinton and members of Congress to establish a national goal of completing 1 million energy-efficient mortgages on existing homes in 1996. The letter also asks the President and Congress to remove "the known 
barriers" to EEMs at Fannie Mae, Freddie Mac, HUD/FHA, and the DVA. The letter would also ask that an "energy-efficient mortgage czar" be appointed to have the responsibility and authority to carry this out. The group prepared a draft of this letter on February 22, 1993. Current plans are to forward a modification of this letter, signed by a group of those concerned, as soon as possible, asking for White House supervision of a national program. A draft of the letter went to the White House in May 1993.

- The grantees also completed a "back-of-the-envelope" analysis showing their position on the effects of a national EEMs program. 'They said that if 1 million EEMs were achieved in 1996, 195 trillion Btu would be saved, 3.6 million tons of carbon dioxide emissions would be avoided, and 125,000 jobs would be created, at no cumulative cost to the U.S. Treasury. The analysis stated that $90 \%$ of existing homes are energy inefficient. It stated that each year in the United States, 8 million mortgages are processed (half for purchase and half for refinance). This represents the potential market for EEMs. The analysis goes on to state that achieving the 1 million EEM goal is feasible.

- Mr. Jim Curtis of the association also provided testimony before a congressional committee working on national energy legislation and has worked actively to promote a national EEMs program.

\section{National Association of REALTORS (NAR)}

- $\quad$ NAR conducted an informal discussion on the Blueprint at its November 1992 annual convention. They asked members to describe what action they wanted NAR to take and how much time NAR should spend on the HERS/EEMs issue.

- A NAR staff member (Steve O'Connor) briefed the NAR Residential Finance Subcommittee on the HERS/EEMs process in mid-January 1993.

- NAR is awaiting HUD's announcement on the five-state EEMs pilot program required under the Energy Policy Act of 1992. Once the NAR staff and members have had time to review the specifics of the pilot program, NAR will consider options for its involvement. NAR had hoped to discuss these options at its April 23-24, 1993, meeting in Washington, D.C.; however, the HUD report was not available in time.

- A Fannie Mae representative met with the Residential Finance Subcommittee during NAR's April 1993 meeting to explain how the Fannie Mae pilot program will work pursuant to the 946 HUD Task Force Report.

- $\quad$ NAR participated in the HERS Council organizing meeting on March 15, 1993.

\footnotetext{
${ }^{1}$ This analysis of effects has not been confirmed by independent analytical studies.
} 


\section{Remodelers}

\section{National Association of the Remodeling Industry (NARI)}

- NARI included articles on the Collaborative and the Blueprint in the Federal Issues Booklet distributed to its Board of Directors three times a year.

- NARI staff plan to pursue membership education and promotion of HERS and EEMs once uniform national guidelines are in place. 


\section{States}

\section{California Home Energy Efficiency Rating System, Inc. (CHEERS)}

- CHEERS has created a Mortgage Subcommittee to assist in developing and introducing a program that will financially assist consumers interested in purchasing energy-efficiency improvements.

- In April 1992, Pacific Gas and Electric (PG\&E), a member of CHEERS Inc., hosted a formation meeting of a National Home Energy Rating Systems Council. The effort was a result of the National Collaborative's need for a national HERS program and led to the formation of the HERS Council Planning Committee.

- CHEERS has become a member of the HERS Council; David Altscher (representing CHEERS) was elected to the Board of Directors in March 1993.

- Between October 1992 and January 1993, CHEERS tested its HERS software, rating more than 300 homes.

- In February 1993, statewide implementation of the program began in San Jose, CA, where CHEERS plans to conduct about 1,200 ratings during a six-month period. The organization will extend the program to Southern California (the Ontario vicinity is being considered) between June and July 1993 before advancing to other parts of the state.

\section{National Association of State Energy Officials (NASEO)}

The bulk of NASEO's efforts since commenting on the Blueprint involved working with a representative Planning Committee for the HERS Council. Deborah Howard chaired the Planning Committee, which was staffed by D\&R International. NASEO was represented on various subcommittees of the Planning Committee in developing bylaws, making recommendations for the Board of Directors and nominating candidates, crafting a mission statement and goals, and planning the HERS organizing meeting-which took place in March 1993. A NASEO member (Deborah Howard, Arizona) was elected the HERS Council Chair of the Board of Directors. NASEO will continue to be involved in the HERS Council and will represent the needs of states in the HERS/EEMs arena. NASEO member states are moving forward in their efforts to encourage HERS programs.

\section{State HERS Provider Information Network}

Energy Rated Homes of Virginia (ERH of VA) has taken the lead in developing a HERS Provider Information Network Program. The program is coordinating the preparation of a manual for state officials and others on how to initiate a HERS program. The manual will be a loose-leaf notebook with sections on such topics as rater training, marketing planning, and certifying rating systems. One of the strengths of this effort is that 43 states, the United Kingdom, and Canadian provinces are contributing materials to the manual. Therefore, its users will be able to avail themselves of information and ideas in a variety of locales, which they can, in turn, adapt to their own local circumstances. In addition, the manual will provide them with points of contact for more information. ERH of VA published the manual in late May 1993. 
The participating organizations include

\begin{tabular}{|c|c|c|}
\hline Alabama & Montgomery & $\begin{array}{l}\text { Alabama Department of Economic and Community } \\
\text { Affairs-Science Technology and Energy Division }\end{array}$ \\
\hline Alaska & Anchorage & Energy Rated Homes of Alaska \\
\hline Arkansas & Little Rock & Energy Rated Homes of America \\
\hline Arizona & Phoenix & Department of Commerce Energy Office, AZ \\
\hline \multirow[t]{4}{*}{ California } & Palo Alto & Bay Area Energy Consultants \\
\hline & San Francisco & Pacific Gas and Electric \\
\hline & Costa Mesa & $\begin{array}{l}\text { CA Home Energy Efficiency Rating Systems, Inc. } \\
\text { (CHEERS, Inc.) }\end{array}$ \\
\hline & Sacramento & California Energy Extension Service \\
\hline Colorado & Denver & Colorado Office of Energy Conservation \\
\hline Connecticut & Hartford & Office of Policy and Management \\
\hline Delaware & Dover & Division of Facilities Management \\
\hline \multicolumn{3}{|l|}{ District } \\
\hline of Columbia & Washington & Alliance to Save Energy \\
\hline Florida & Tallahassee & Florida Energy Office \\
\hline Georgia & Atlanta & $\begin{array}{l}\text { Southern Electric International Good Cents Division } \\
\text { Georgia Office of Energy Resources }\end{array}$ \\
\hline Indiana & Indianapolis & Department of Commerce Energy Policy \\
\hline Illinois & Champaign & Home Energy Rating System of Illinois \\
\hline Iowa & Des Moines & Department of Natural Resources \\
\hline Kansas & Topeka & Department of Commerce and Housing \\
\hline Kentucky & Frankfort & Department of Natural Resources \\
\hline Louisiana & Baton Rouge & Department of Natural Resources \\
\hline Maine & Augusta & Department of Economic and Community Development \\
\hline \multirow[t]{4}{*}{ Maryland } & Annapolis & Maryland Energy Administration \\
\hline & Baltimore & Baltimore Gas and Electric \\
\hline & Baltimore & Energy Conservation Management, Inc. \\
\hline & Bethesda & Conservation Management Corporation \\
\hline Massachusetts & Boston & Western Mass Electric Energy Crafted Homes \\
\hline Michigan & Lansing & Michigan Office of Energy Programs \\
\hline \multirow[t]{2}{*}{ Minnesota } & St. Paul & Energy Division \\
\hline & St. Louis Park & Minnegasco \\
\hline Mississippi & Jackson & Energy Rated Homes of Mississippi \\
\hline Missouri & Mountain View & Energy Rated Homes of Missouri \\
\hline Montana & Helena & $\begin{array}{l}\text { Montana Department of Natural Resources and } \\
\text { Conservation }\end{array}$ \\
\hline \multirow[t]{2}{*}{ New Jersey } & Newark & Public Service Electric and Gas \\
\hline & Trenton & $\begin{array}{l}\text { New Jersey Department of Environmental Protection } \\
\text { and Energy }\end{array}$ \\
\hline New Mexico & Santa $\mathrm{Fe}$ & Energy, Minerals, and Natural Resources Department \\
\hline \multirow[t]{2}{*}{ New York } & Albany & New York State Energy Office \\
\hline & Albany & NYSE-Star \\
\hline \multirow[t]{2}{*}{ North Carolina } & Raleigh & Dept. of Economic and Community Development \\
\hline & Raleigh & Division of Energy \\
\hline Ohio & Columbus & Department of Development \\
\hline Oklahoma & Oklahoma City & Oklahoma Department of Commerce \\
\hline Oregon & Salem & Oregon Department of Energy \\
\hline
\end{tabular}




\begin{tabular}{|c|c|c|}
\hline \multirow[t]{3}{*}{ Pennsylvania } & Harrisburg & Energy Office \\
\hline & Philadelphia & Philadelphia Gas Works \\
\hline & Philadelphia & Grace Energy Center \\
\hline Rhode Island & Providence & Rhode Islanders Saving Energy (RISE) \\
\hline South Carolina & Columbia & Governor's Energy Division \\
\hline South Dakota & Pierre & Governor's Office of Energy Policy \\
\hline Texas & Austin & Texas Governor's Energy Management Center \\
\hline Vermont & Burlington & Energy Rated Homes of Vermont \\
\hline Virginia & Richmond & Energy Rated Homes of Virginia \\
\hline Washington & Olympia & Washington State Energy Office \\
\hline West Virginia & Charleston & West Virginia Fuel and Energy Office \\
\hline Wisconsin & Madison & Department of Administration Energy Bureau \\
\hline \multicolumn{3}{|c|}{ Outside the United States } \\
\hline British Columbia & Vancouver & BC Hydro \\
\hline Canada & Toronto, Ontario & Ministry of Energy \\
\hline UK, Scotland & Edinburgh & Energy Education, Ltd. \\
\hline
\end{tabular}

These organizations will be contributing materials and will also be receiving the manual when it is published. 


\section{Utilities}

\section{American Gas Association (AGA)}

- An AGA staff member (Mary Carson) prepared an industry "Issues Analysis" on the National Collaborative and the HERS Council and sent it to AGA residential marketing managers in midFebruary 1993.

- A feature article on energy-efficient mortgages and the HERS Council is scheduled to run in December 1993 in the American Gas magazine, which has a distribution of 5,300.

- Staff members discussed with member companies the importance of running a seminar on HERS/EEMs.

- In November 1992, a staff member attended the National Association of Realtors conference and handed out copies of the Executive Summary of the Blueprint to those who visited the AGA Energy Break Room.

- Staff members are preparing a manual called It Takes Energy to Sell a House that will contain sections on EEMs and HERS; it will be released in summer 1993.

- $\quad$ AGA has participated in the Planning Committee for the HERS Council; an AGA representative (Mary Carson) was elected to the HERS Council Board of Directors at the March 15, 1993, organizing meeting.

\section{American Public Power Association (APPA)}

- APPA reported the outcome of the Collaborative to its members and continues to promote the development of HERS through education courses, conferences, and the trade press.

- $\quad$ APPA provided an update of the HERS/EEMs process at its October 1992 Energy Services Conference in Boston, which brought together several hundred utility DSM managers.

- APPA profiled the Blueprint in their weekly publication and published an article on the Fort Collins' HERS program.

- $\quad$ APPA will offer a session on HERS/EEMs at the June 1993 APPA National Conference attended by utility general managers and CEOs.

- $\quad$ HERS will be a topic of discussion at a DSM implementation course planned for September 1993.

- $\quad$ APPA has noted increased interest in HERS among utilities. As of March 1993, more than 150 public power utilities operate HERS programs.

\section{Edison Electric Institute (EEI)}

The Three-E Program (for efficiency, environment, and electricity) is a full electric industry initiative that provides electric utility leadership on these issues. The initiative is jointly funded by EEI and the Electric Power Research Institute (EPRI). It includes active participation (including financial) by 40 investor-owned utilities and represents $48 \%$ of residential customers 
nationwide. The program will create and promote a label that will be used to certify utility programs that exemplify efficiency and environmental quality criteria.

First-phase activities have been to develop guidelines and communicate the benefits and attributes of the program. These include stressing not only the environmental and energy-efficiency aspects, but also the benefits of consumer savings and added value. The program will not attempt to replicate a rating in terms of a uniform scale. Rather, it will imply a certain level of efficiency. The program will be actively seeking ways to integrate with other ongoing programs and groups in these areas.

- EEI presented the Three-E Program at the annual Building Environment and Thermal Envelope Coordinating Council Conference in November 1992.

EEI gave a presentation on the Three-E project as part of the session on "DSM Beyond Conservation" at the 6th National DSM Conference, March 1993.

EEI briefed EPA on the Three-E project and received its support and indication of interest to participate.

- $\quad$ EEI was asked to join the HERS Council Planning Committee in December 1992.

- Tom Farkas of EEI was elected to serve on the board of the HERS Council in March 1993.

- $\quad$ Rick Tempchin of EEI moderated a workshop at the EEBA annual conference in March 1993. The workshop was on the future of the utility industry and addressed HERS and EEMs.

- Tom Farkas of EEI presented information on the HERS Council and a uniform rating methodology to the EEI Residential Marketing Committee March 1993 meeting.

EEI prepared an article on the HERS Council, which appeared in EEl's Division Diary in March 1993.

The Three-E project is halfway through Phase I, and the energy-efficiency and environmental criteria are being finalized. 
Appendix C

Summary of Organizational Comments on A Blueprint for Action 


\section{Organizations Commenting}

American Association of Retired Persons (AARP)

American Public Power Association (APPA)

Alliance to Save Energy (ASE)

American Society of Home Inspectors (ASHI)

Appraisal Institute (AI)

Consumer Federation of America (CFA)

CHERS, Inc. (CHERS)

Energy Efficient Builders Association (EEBA)

Edison Electric Institute (EEI)

Energy Rated Homes of America (ERHA)

Farmers Home Administration (FmHA)

Federal Housing Administration (FHA)

Federal Home Loan Mortgage Corporation (Freddie Mac)

Federal National Loan Mortgage Corporation (Fannie Mae)

Mortgage Bankers Association (MBA)

National Association of Home Builders (NAHB)

National Association of REALTORS (NAR)

National Association of the Remodeling Industry (NARI)

National Association of State Energy Officials (NASEO)

Natural Resources Defense Council (NRDC)

Southern Electric International, Good Cents Division (SEI)

\section{Not Commenting}

American Gas Association (AGA)

Appraisal Subcommittee, Federal Institutions Examination Council (editorial comments only)

Association of Energy-Efficient Mortgage Service Companies (AEEMSC) (informal comments only)

U.S. Department of Veterans Affairs (DVA) 


\section{Alliance to Save Energy (ASE)}

ASE is "in full agreement with [DOE's] assessment of the results of the $B F A^{1}$ contained in the April transmittal letter that "we got 75 percent of the way." ASE said that the last 25 percent, containing the more difficult issues, will ultimately determine the success of EEMs/HERS. "The momentum and education process which [DOE] began must continue so that all stakeholders realize the opportunities, are aware of the issues, and can become part of the solution."

\section{American Association of Retired Persons (AARP)}

No formal comments on the $B F A$ are planned at this time.

\section{American Gas Association (AGA)}

AGA has decided not to comment on the $B F A$ at this time.

\section{American Public Power Association (APPA)}

APPA said that the $B F A$ is a step in the right direction to increase the nation's energy efficiency. APPA stated that "the HERS industry must be sensitive to concerns of primary and secondary mortgage lenders, especially regarding the rate of defaults associated with EEMs." APPA favored the initiation of pilot projects "to determine the mortgage default impacts of higher lending ratios to improve home energy efficiency."

\section{American Society of Home Inspectors (ASHI)}

ASHI has submitted no comments on the BFA.

\section{Appraisal Institute (A)}

Mr. Lennhoff said that "the primary valuation issue relative to energy improvements" is properly addressed in the $B F A$. He said that the "correct adjustment amount must represent the improvements' contribution to the property's value and is not necessarily equal to their cost."

\section{Appraisal Subcommittee, Federal Institutions Examination Council (FIEC)}

Mr. Baker provided editorial comments only.

\section{California Home Energy Rating System, inc. (CHERS) ${ }^{2}$}

CHERS applauds the goals, efforts, and accomplishments of the Collaborative for developing a "blueprint" which serves as a road map for linking HERS and EEMs and for promoting and fostering affordable, comfortable and energy-efficient housing throughout the United States.

It is of paramount importance for the architects and participants that have developed the Blueprint to ensure agreement is reached between the EEM and HERS segments of the Collaborative and that the BFA

\footnotetext{
${ }^{1} B F A$ stands for A Blueprint for Action.

${ }^{2}$ Now named CHEERS, Inc.
} 
moves on to serve as a living document which assures HERS and EEMs are coupled and become a consumer-useable reality.

To that end, CHERS is developing a program which is believed to meet the $B F A$ goals of a combined HERS/EEM in California as well as a commitment for ongoing support for the BFA's goals.

\section{Consumer Federation of America (CFA)}

CFA recommends the development and implementation of a public education program. The objective of the program should be to make the country aware of the availability of EEMs and how to access them.

\section{Edison Electric Institute (EEI)}

EEI commended DOE for initiating the Collaborative and providing leadership to bring together interested stakeholders to address HERS and EEMs. EEI joined the Collaborative with the objective of increasing both the efficiency and the affordability of housing. "Much has been accomplished by the Collaborative toward that end, while much more is yet to be achieved."

EEI said that, although there was significant agreement on a number of issues, there is a need to shift attention to "an entirely new macro approach," to remove barriers to a national program, such as incentive regulation for energy efficiency improvements in demand-side management programs.

EEI said that utilities will continue to work with other stakeholders toward creating uniform guidelines and a uniform methodology for linking HERS with EEMs, and to make such guidance widely available without the need for public subsidies.

EEI said its most important comments on the BFA related to (1) the linkage between HERS and EEMs, (2) a uniform rating methodology, (3) self-supporting programs, (4) the significance of a nationally recognized consensus standard, (5) clarification of "basic energy use," and (6) the accuracy of HERS information on recommended energy improvements.

\section{Energy Efficient Building Association (EEBA)}

EEBA said that the overall Collaborative effort was positive and that consensus among such a large group is difficult.

EEBA's comments are aimed at two specific areas of the Blueprint. Under the section on lender indemnification, EEBA has not seen any evidence to substantiate the claim that the mortgage lending community may be at added risk from financing the cost of energy-efficiency improvements. They believe the opposite is more likely true as families with energy efficient homes are protected from rising energy costs and therefore more able to cope with their mortgage payments. EEBA believes that this issue should not pose a hindrance to implementation of a national EEMs/HERS effort.

Under the section on implementation, EEBA said that training and implementation were not fully addressed. EEBA said that training opportunities and a common set of guidelines should be made available to builders and others to ensure awareness, availability, consistency, and the overall success of implementation.

\section{Energy Rated Homes of America (ERHA)}

ERHA was critical of the Review Draft. ERHA said that the BFA characterized the HERS industry as in disarray and without technical credibility. ERHA saw the BFA as calling for DOE to take charge and 
decide what needs to be done and how; ERHA disagrees that this would be the best approach. ERHA said the $B F A$ did not reflect the Collaborative's agreements: "The first part is not accurate and the last part is not consistent with our understanding of the conclusion or intent of the collaborative."

ERHA said it feared that the BFA invites federal agencies to develop a HERS tool "to bring order to the alleged confusion." ERHA wants a HERS Industry Council "to take charge of many of these issues." DOE, the organization said, should support, rather than control, the HERS industry.

ERHA said the BFA could be enhanced if the implementation and conclusion sections were made more comprehensive with defined goals, a "directed plan," and a unified commitment. ERHA provided detailed editorial comments on the Review Draft.

\section{Farmers Home Administration (FmHA)}

The $B F A$ is a culmination of the Collaborative's efforts to develop a voluntary national program that encourages energy efficiency in housing by promoting mortgage incentives based on home energy savings. While the $B F A$ establishes a broad framework for this program, it also identifies several key issues where the Collaborative "agreed to disagree." Hopefully, the momentum of the Collaborative's effort will continue and bring a national program to fruition.

\section{Federal Home Loan Mortgage Corporation (Freddie Mac)}

"Freddie Mac recommends the Blueprint for Action be issued as currently written. It accurately portrays the issues on which the Collaborative achieved a consensus, as well as those on which lending and nonlending members agreed to disagree. Naturally, we all would have preferred to have achieved complete consensus on all aspects of the issue, but neither the lenders nor the non-lenders are likely to change their positions on the areas of disagreement. In any case, this should not obscure the progress the Collaborative made in developing a common approach to a voluntary national EEM program."

\section{Federal Housing Administration (FHA)}

Pending.

\section{Federal National Mortgage Association (Fannie Mae)}

"The $[B F A]$ realistically reflects the differing views on certain key issues critical to the mortgage process, such as collateral value and borrower qualification. We believe that any uniform approach to the energyefficient mortgage must take into account the need to manage risk in a prudent manner, consistent with our commitment to safety and soundness."

\section{Mortgage Bankers Association (MBA)}

MBA congratulated the DOE for an accurate report summarizing the Collaborative process. MBA's positions on and concerns with several of the specific proposals advanced by some members of the Collaborative are included in Chapter Three, Section IV of the report. These concerns are also included in the report's companion volume, Going National with HERS and EEMs: Issues and Impacts.

\section{National Association of Home Builders (NAHB)}

NAHB said it was impressed with the "thoughtful and thorough examination of issues in the Report," given the complexity of the task. 
NAHB has "grave misgivings" about two of the $B F A$ 's statements:

(1) NAHB does not support the concept of indemnifying lenders against any added risk of borrower default. NAHB said they knew of no evidence, either statistical or anecdotal, to suggest that the default experience of EEMs is worse than other mortgages. NAHB said they believe that the opposite is true.

(2) NAHB said that the BFA did not differentiate sufficiently between new and existing housing. NAHB does not believe it is necessary to conduct a HER as a precondition to providing an EEM to the buyer of an energy efficient new home. NAHB said that compliance with the thermal performance requirement of mandatory property standards should be certified by builders and that these certifications should be sufficient to qualify buyers for an EEM.

NAHB said that these issues are of such vital concern to their members that they are unable to approve the $B F A$ as drafted.

\section{National Association of Energy Efficient Mortgage Service Companies (NAEEMSCo)}

Membership reaction is favorable. The members said they were pleased that the agencies will hold fast to their consensus agreements on pages 8 and 9 in the $B F A$. They want implementation of a workable EEM. The members are waiting to see what Fannie and Freddie will do to make their EEM programs workable for the buyers and owners of existing homes when those homes are not as energy efficient as they could or should be.

\section{National Association of REALTORS (NAR)}

The NAR Board of Directors supported the development of a uniform HERS system and the development of a means to recognize energy savings in the mortgage finance transaction. NAR believes that a uniform HERS system is the first and primary step for establishing a workable HERS/EEMs program, and that participation in the HERS/EEMs system should be entirely voluntary.

NAR stated there should be no "unreasonable implicit or explicit legal liabilities" associated with marketing HERS-rated properties. They said that the HERS system adopted should not permanently disadvantage properties that are not rated as energy efficient. NAR also wanted equal availability to borrowers of the recognition of energy savings in the mortgage finance transaction for existing and new housing.

\section{National Association of the Remodeling Industry (NARI)}

NARI stated that remodelers would be more likely to benefit from a program involving home improvement loans. However, NARI said that remodelers would benefit if the home buyers knew in advance that EEMs were available. Remodelers themselves would not market the program except to existing homeowners in need of substantial energy savings repairs.

NARI strongly favors training and promotional programs to educate lenders, real estate professionals, and consumers about the availability of EEMs. NARI would like to see the development of qualified remodeling contractor referral systems so remodelers could consult with potential home buyers prior to purchase. 


\section{National Association of State Energy Officials (NASEO)}

NASEO said that the Collaborative established a network through which substantive issues could be addressed. The $B F A$ was not a final protocol allowing states to embark upon individually developed rating systems. NASEO said that the overall expectations and needs of the states for a blueprint for developing rating systems and a clear recommendation on what energy-efficient mortgage process should be were not fully met. Examples of areas not fully addressed were the rating scale formulation, common breakpoints where a structure would become eligible for an energy-efficient mortgage, and energy needs for comfort conditioning in various climates.

\section{Natural Resources Defense Council (NRDC)}

NRDC endorsed the $B F A$ and said that a nationally available, effective, and user-friendly HERS/EEMs program could promote both housing affordability and energy efficiency, while helping to preserve the environment. NRDC said it was disappointed that the Collaborative did not go further in achieving consensus on national HERS/EEMs implementation proposal. NRDC said that the recommendations were not as specific nor as aggressive as the organization would ideally have hoped.

\section{Southern Electric International, Good Cents Division}

(1) Concerns exist about the task of developing technical standards for HERS. HERS program certification process should remain voluntary. The development of a "closed-ended" scale would limit the recognition of energy-efficiency features that go beyond requirements of codes or programs. The HERS calculational tool's capability to determine accurate energy-usage and costs should be the most important concern of the guidelines developed for uniform rating. The "single rating method" discussion should be revisited for clarity. The expense of setting up a national data base should be considered during the implementation phase.

(2) Good Cents utilities indicated willingness to share the utility program data available to illustrate the value of energy-efficient homes. Indemnification for perceived risk by a third party drew interest from some utilities.

Although many utilities offer EEM training, the Good Cents utilities do not believe that training will mean that implementation of EEMs is more likely to occur.

\section{U.S. Department of Veterans Affairs (DVA)}

The DVA is not planning to make any formal response to the $B F A$. 
Appendix D

Recent Articles on the National Collaborative, HERS, and EEMs

(1991-1993) 


\section{Recent Articles on the National Collaborative,} HERS, and EEMs, 1991 - 1993

Allan L. Frank Associates. 1992. "DOE Announces Consensus Reached by National Collaborative on HERS, EEMs." The Solar Letter, 2 (7):71. April.

Allen, Linn. 1992. "Energy-Efficient Mortgage May Be Ready to Catch On." The Indianapolis Star, Section AGE, p. I-6. May 3.

1992. "Energy Loans Get New Boost." The Chicago Tribune, p. 1K. March 15.

Andrews, Steve. 1992. "Energy Mortgages Energize Sales." Builder, p. 44. May

Bach, Matthew. 1992. "How Does Your Home Heat? Energy-Efficiency Ratings Grant Aid Homeowners." Daily News, p. 1. August 27.

Beneroff, Eric. 1992. "Disagreement and Confusion Slow Availability of Energy-Efficient Mortgages." Professional Builder and Remodeler, pp. 117-118. April.

Brack, Richard. 1992. "Energy-Efficient Loan Helps New Home Buyers." The Des Moines Register, Metro Home/Real Estate Section. December 11.

Braunstein, Leslie. 1992. "Energy-Efficient Mortgages: A Utility Perspective." Public Utilities Fortnightly. July 1.

CertainTeed Home Institute. 1992. How to Get the Home You Want. (Brochure). Valley Forge, Pennsylvania: CertainTeed Home Institute. July.

Cutter Information Corporation. 1993. "New York State Offers $\$ 4,000$ Per House to Energy-Efficient Home Builders." Energy Design Update, 13(4): 1. April.

Duncan, Russell. 1992. "A Winning Alliance: Providing Realtors with a Training Program on the Value of Energy-Efficient Homes Helps Carolina Power \& Light, Real Estate Agents and Home Buyers." Division Diary, p. 42. September/October.

Edison Electric Institute. 1992. "Three E Program Advances." Division Diary, p. 4. Spring.

1992. "Three E Program Advances." Division Diary, p. 42. September/October.

Energy Conservation Digest. 1992. "Freddie Mac Unveiling 'Aggressive' New Plan for Energy Conservation." Washington, D.C.: Energy Conservation Digest. 15(11):101. June 1.

Energy Rated Homes of Alaska. A Consumer's Guide to a Home Energy Rating. (Brochure). Anchorage, Alaska: Energy Rated Homes of Alaska.

For Alaskans Who Don't Have Money to Burn: The Smart Way to Buy or Sell a Home. (Brochure). Anchorage, Alaska: Energy Rated Homes of Alaska.

Energy Rated Homes of America. 1990/1991. "DOE Commits to Collaboration." Home Energy Rating News. 3(1):1. Winter. 
. 1991. "HERS Industry Meeting." Home Energy Rating News. 3(2):1. Spring.

. 1991. "Consensus Collaborative Committee Begins Work." Home Energy Rating News. $3(2): 1$. Spring.

Summer.

1991. "CCC Update: Broadbased and on Track." Home Energy Rating News. 3(3):1.

Energy Rated Homes of Vermont. 1992/1993. "Utilities Offer Energy Ratings As Incentive for Efficient Construction." The Energy Efficient Mortgage Newsletter. 5:1. Winter.

1992/1993. "Innovative Pilot Program May Be in Vermont's Future." The Energy Efficient Mortgage Newsletter. 5:1. Winter.

1992/1993. "Energy Efficient Mortgages Are a Hot Topic in D.C." The Energy Efficient Mortgage Newsletter. 5:1. Winter

1992/1993. "Task Force 'Blueprint for Action' Paves the Way for National Action." The Energy Efficient Mortgage Newsletter. 5:1. Winter.

Faesy, Richard. 1992. Lessons Learned from Four Years of Operating a Home Energy Rating System and Energy Efficient Mortgage Program. Burlington, Vermont: Energy Rated Homes of Vermont. March.

Farkas, Tom. 1991. "Special Mortgages Worth Banking On: Home Builders and Utilities Working Together Can Take the Lead in Expanding the Use of Energy-Efficient Mortgages." Energy Focus-Residential Magazine.

Figueroa, Javier H. 1992. Memorandum to National Collaborative Members re: Energy Efficient Mortgage (EEM) update. Olympia, Washington: Washington State Energy Office. October 16.

Greengard, Samuel. 1992. "Energy Boost." p. 36. September.

Harney, Kenneth R. 1992. "Freddie Mac's Energy-Saving Incentives." Washington, D.C.: Washington Post, p. F1. May 5.

JWB Mortgage. 1993. "Stretch Mortgage Dollars with Energy Efficiency," pp. 29-31. July.

Johnson, Wayne. 1992. "Sell Customers the Multiple Benefits of Efficiency." Air Conditioning \& Refrigeration News, pp. 5-6. May 18.

Lehman, Jane H. 1992. "Study Shows Ways to Boost Energy-Efficient Housing: Ratings, Mortgage Incentives are Key." Washington, D.C.: Washington Post, p. E1. April 18.

1992. "Proposals Seek to Set Plumbing Product Standards." Washington, D.C.: Washington Post, p. E4. August 8.

Martin, Randy. 1991. "More for Your Money." Iowa Conservationist, pp. 12-15. July.

McClintock, Mike. 1992. "Rating Houses' Efficiency: Pros and Cons of a National Comparison Scale." Washington Post, p. 7. June. 
1992. "Rating for Energy Use Debated." San Francisco Chronicle, p. 87. August 5.

McDonnell, Stephen S. 1992. "Energy Education: Utilities are Informing Realtors about the Benefits of Energy-efficient Homes." Division Diary, p. 28. July/August.

1992. "Adding EEMs to Your Business Plan Toolbox." Good Cents Magazine. 2(1):8-11. January/February.

Mortgage Bankers Association. 1992. "Task Force Proposes National Energy-Efficient Mortgage Program." MBA StateLines. 5(7):1. July.

1992. "Vermont/Fannie Mae to Offer Energy Efficient Mortgage Program." MBA StateLines. 5(7):1. July.

The National Energy Specialist Association. 1991. "DOE/HUD Propose Collaborative on HERS \& EEMs." The NESA Newsletter, p. 1. May.

New York State Builders Association. 1993. "Buying a NYSE-Star Home Made Easier-Thanks to Fannie Mae!" NYSE-Star, p. 2. Spring.

Office of Energy Policy. 1993. It's Coming! Energy Rated Homes of Indiana. (Brochure). Indianapolis, Indiana: Indiana Department of Commerce. January.

Pacific Gas \& Electric. 1992. California Home Energy Efficiency Rating System (CHEERS) PG\&E Employee Fact Sheet. (Flyer).

American Public Power Association. 1992. "Panel Pushes Energy Mortgages but Lenders Worry About the Risks." Public Power Weekly. Washington, D.C.: American Public Power Association. April 20.

Rhode Island Housing and Mortgage Finance Corporation. 1993. "New Mortgage Program Saves Buyers on Energy and Closing Costs." Rhode Island Housing News Release. March.

Shaman, Diana. 1993. Energy-Efficient Houses, with a Bonus." The New York Times. April 18.

Tarrant, Bert. 1992. "Alaska Home Energy Program United with Federal Mortgage Plan." Anchorage Times, p. D1. February.

U.S. Department of Energy. 1992. "A Reorganized Office of Conservation and Renewable Energy: Moving Advanced Energy Technologies into the Marketplace." DOE This Month. 15(7):8. July.

. 1992. "DOE to Spearhead Effort to Encourage Use of Energy-Efficient Home Mortgage Programs." Press Release. Washington, D.C.: Department of Energy. March 24.

Western Area Power Administration. 1992. "Energy-Efficient Mortgages Benefit Everyone." Customer $C \& R E$ Management Update. August.

1992. "Home Energy Rating Systems." Customer C\&RE Management Update.

Wise, Christy. 1992. "Energy Efficient Mortgages Get a New Lift." Real Estate Finance Today, p. 2. July 6 . 
Appendix E

Text of A Blueprint for Action 
A NATIONAL PROGRAM FOR ENERGY-EFFICIENT MORTGAGES AND HOME ENERGY RATING SYSTEMS:

A BLUEPRINT FOR ACTION

\section{REVIEW DRAFT}

Final Report of the Nationai Collaborative on

Home Energy Rating Systems and Mortgage Incentives

for Energy Efficiency

Prepared by the

National Renewable Energy Laboratory

Washington, D. C.

March 1992 


\section{Preface}

This Review Draft reports findings and recommendations of the National Collaborative on Home Energy Rating Systems and Mortgage Incentives for Energy Efficiency. The U.S. Department of Energy, in cooperation with the U.S. Department of Housing and Urban Development, formed this National Collaborative as a National Energy Strategy initiative. Participating in the Collaborative were representatives of the primary and secondary mortgage markets, builder and remodeler organizations, real estate and appraiser associations, the home energy rating system industry, utility associations, consumer and public interest groups, state and local government interest groups, and environmental organizations. The Collaborative's purpose was to develop a voluntary national program encouraging energy efficiency in homes through mortgage incentives linked to home energy ratings.

The National Collaborative wrote $A$ Blueprint for Action, which was published by the National Renewable Energy Laboratory (NREL).

A companion volume to this one, Going National with HERS and EEMs: Issues and Impacts (NREL/TP261-4706), provides technical documentation to the Collaborative's efforts. Also published by NREL, it contains a collection of 55 technical issue papers and 13 special papers prepared by the members of the Collaborative's Consensus Committee and Technical Advisory Committees. In addition, Going National contains a bibliography and glossary, which were prepared by Collaborative members and staff.

The valuable contributions of the Collaborative's members, those who served on technical committees. and staff are acknowledged. Without their dedication and commitment to a challenging task, the accomplishments reflected in this report would not have been realized. 


\section{Executive Summary}

Cost-effective energy efficiency technologies ${ }^{*}$ exist today to reduce dramatically the $\$ 100$ billion we spend each year on energy for our nation's housing. However, adoption of these technologies has been far slower than would be economically best for our nation and for individuals. One reasun is that builders and home buyers have a strong tendency to minimize the "up-front" cost of a residential property, even at the expense of future savings. Mortgage loan practices reinforce this tendency. They fail to consider the lower total cost of owning an energy-efficient home when energy expenses are added to mortgage and tax payments.

One of the goals of the National Energy Strategy, recently developed at the direction of President Bush. is to counteract this tendency by developing reliable methods for rating the energy performance of residences and by encouraging mortgage-lending practices that fully reflect the value of lower energy operating costs. In response to that call for action, the U.S. Department of Energy, in cooperation with the U.S: Department of Housing and Urban Development, convened the National Collaborative on Home Energy Rating Systems and Mortgage Incentives for Energy Efficiency. The National Collaborative. comprised members representing 25 organizations in the housing, mortgage finance, and energy supply industries, along with state and federal government, federally chartered financial institutions, and public interest organizations. Four technical advisory committees supported their work.

The mission of the National Collaborative was to reach a consensus on a voluntary national program that will link credible home energy rating systems with mortgage incentives for energy-efficient housing. A Blueprint for Action reports on the National Collaborative's findings and recommendations for creating such a program.

Widespread availability of energy-efficient mortgages (EEMs), teamed with accurate home energy rating systems (HERS), would make it easier and more affordable for Americans to live in energy-efficient homes. In fact, the Joint Center for Housing Studies has estimated that 250,000 more U.S. families could become first-ime homeowners each year with EEMs. And the benefits to the environment of increasing the energy efficiency of the nation's housing stock would be significant.

In theory, a national EEM program would make available home mortgages that take into account the energy cost savings of the home. Home buyers could apply for EEMs when purchasing an energyefficient home or when they are buying an existing home and planning to make immediate energy improvements to it. EEMs would have more favorable terms and qualifying conditions than conventional

\footnotetext{
" Energy efficiency" is intended to include both energy efficiency measures (such as insulation and lowemissivity windows) and renewable energy technologies (such as passive solar design and solar domestic hot water systems), the use of which reduces a home's consumption of utility-supplied energy.
} 
loans. Supporting the EEMs, a reliable HERS allows both the lenders and the home buyers to be confident of the predicted energy cost savings.

EEMs and HERS are not new. However, EEMs have not been widely used because most buyers and many lenders are not aware of them. Also. lenders are not completely confident of the various EEM programs. Extra paperwork, lack of uniformity among EEM programs, and uncertainty about promised energy cost savings make lenders reluctant to use EEMs. In addition, lenders usually do not have access to a credible HERS program to determine energy cost data.

The members of the National Collaborative examined these issues and worked hard to hammer out a strategy to deliver an effective EEM program linked to voluntary HERS. There was broad consensus on the actions that can be taken immediately to set the stage for the program:

- Develop common standards for EEMs among the five federal agencies and federally chartered financial institutions: the Federal National Mortgage Association (Fannie Mae), the Federal Home Loan Mortgage Corporation (Freddie Mac), the Federal Housing Administration (FHA), the Department of Veterans Affairs (DVA), and the Farmers Home Administration (FmHA)

- Review the procedures of government programs and government-chartered institutions to remove any unnecessary barriers to implementing EEMs and simplifying the loan process

- Develop training and promotional programs to educate lenders, builders, real-estate professionals, appraisers, and consumers about EEMs

- Collect and analyze data from existing EEM programs on use patterns, default rates, and other factors.

When considering the design and implementation of HERS, the National Collaborative members concluded that the following steps should be taken:

- Develop a reliable, accurate, nationally uniform HERS program that could be used on a voluntary basis

- Implement HERS through state and local programs

- Train appraisers, real-estate personnel, energy raters, and others to use the system

- Instinute quality control mechanisms for HERS.

The National Collaborative members agreed on several desirable characteristics of a national EEM program, including:

- All housing will be financed in the program

- The underwriting process will adequately recognize the reduced operating costs of energy-efficient housing

- Mortgage financing of both energy-efficient construction and improvements will be permitted

- Lenders will be indemnified against any added risk of borrower default 
- Lenders will be able to use HERS to provide a reliable technical basis for allowing underwriting of mortgage loans by providing energy cost savings information to lenders.

However, National Collaborative members were not able to reach consensus on some specific provisions of a national EEM program. such as how energy-efficiency improvements or construction costs should be reflected in the mortgage or how to incorporate energy cost savings into the procedures used to qualify people for EEMs. On these points, members agreed to disagree. A number of nonlending members advanced a concept for EEM characteristics: mortgage-lending members explained their concerns with the concept. Both sides of these issues are discussed in A Blueprint for Action.

The National Collaborative, through a consensus-building process, has constructed concepts that can link home financing to a successful national program of home energy rating systems. Collaborative members have progressed in understanding each other's needs and concerns that otherwise may have been barriers to creating a national program. Most important, the process has generated a momentum that can mold a successful program to help more people own energy-efficient homes.

Much remains to be done to fully develop, promote, and implement a national program of energyefficient mortgages linked to voluntary home energy rating systems. Many of the report's assumptions and goals need to be carefully tested to provide relevant data that will allow the program to have its maximum impact. These data will provide the basis for completing the unfinished business outlined in this report. But the need for data does not hinder action. Member organizations will review and comment on this Review Draft, which will also be made available for public comment. Implementation strategies will be further developed and tested, both for energy-efficient mortgages and for home energy rating systems. A Blueprint for Action will be revised to reflect the comments of member organizations and the public. 


\section{CONTENTS}

Chapter One: Introduction

I. Background

II. The National Collaborative

III. A Blueprint for Action

Chapter Two: Linking HERS and EEMs $\ldots \ldots \ldots \ldots \ldots \ldots \ldots \ldots \ldots \ldots$

Chapter Three: Energy-Efficient Mortgages $\ldots \ldots \ldots \ldots \ldots \ldots \ldots \ldots \ldots \ldots \ldots$

I. Characteristics of a Proposed Program upon Which There Was Full Consensus ... . 8

II. Consensus Agreements on EEM Issues and Recommended Actions . . . . . . . . . 9

III. Specific Characteristics of an EEM Program Advanced by a Number of

Nonlending Members . . . . . . . . . . . . . . . . . . . . . . 12

IV. The Mortgage-Lending Members Respond $\ldots \ldots \ldots \ldots \ldots \ldots \ldots \ldots \ldots$

Chapter Four: Home Energy Rating Systems $\ldots \ldots \ldots \ldots \ldots \ldots \ldots \ldots \ldots \ldots \ldots$

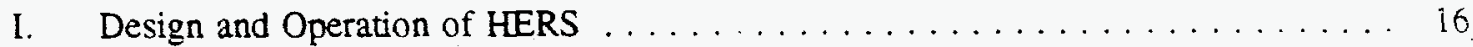

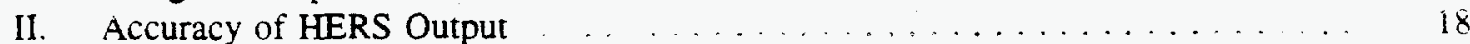

III. Other Recommendations for HERS $\ldots \ldots \ldots \ldots \ldots \ldots \ldots \ldots \ldots \ldots \ldots \ldots$

Chapter Five: Implementation $\ldots \ldots \ldots \ldots \ldots \ldots \ldots \ldots \ldots \ldots \ldots \ldots \ldots \ldots$

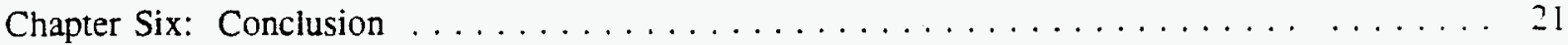

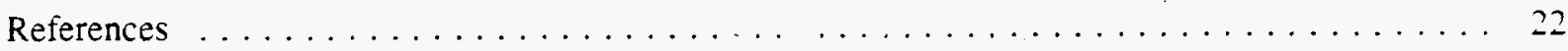

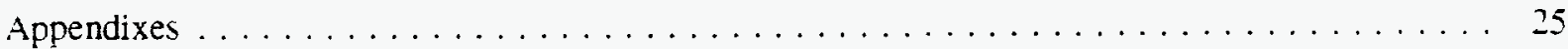

Appendix A: Existing Energy-Efficient Mortgage Programs . . . . . . . . . . . 25

Appendix B: Members of the National Collaborative . . . . . . . . . . . . . . . 29

Appendix C: Assigned Issues for the Technical Advisory Committees . . . . . . . . . 33

Appendix D: National Collaborative Schedule $\ldots \ldots \ldots \ldots \ldots \ldots \ldots \ldots \ldots \ldots \ldots$

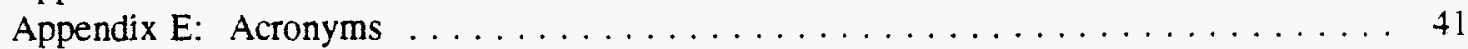

Glossary $\ldots \ldots \ldots \ldots \ldots \ldots \ldots \ldots \ldots \ldots \ldots \ldots \ldots \ldots \ldots \ldots \ldots \ldots$ 


\section{Chapter One: Introduction}

Americans share the belief that their housing should be affordable, comfortable, and energy efficient. But, research has shown that the level of investment in energy efficiency in housing is less than that which is economically justified. Two major barriers limit investment in home energy efficiency. First, energy efficiency represents increased front-end cost. either as a home is first built or as an exiscing home is later improved. It is a long-term investment that must be financed. Second, there are insufficient market data to fully understand the value of energy efficiency in housing.

In recent years, growing attention has been given to two concepts that can help overcome these barriers-home energy raung systems. or HERS. and energy-efficient mortgages, or EEMs. HERS provide objective, standardized information on the energy performance of homes. analogous to miles-per-gallon tatings on automobiles or EnergyGuide labels on appliances. And EEMs provide a financing mechanism for energy efficiency. They allow a buyer of an energy-efficient home to qualify for a higher mortgage, using the future savings in energy costs to afford higher mortgage payments. EEMs may also be used to finance energy-saving improvements to existing homes. as part of the primary mortgage, at the time of purchase or refinance.

Expanded use of HERS could stimulate increased use of energy-efficiency and renewable energy technologies ${ }^{1}$ by making energy efficiency a more qualified, visible, and recognized attribute as homes are designed, built, bought, and improved. It would facilitate informed decision making. At the same time, expanded EEM activity will encourage home builders and owners to invest in energy-saving features without worrying that they are making their properties less affordable. HERS and EEMs have the potential to be closely linked. Energy cost estimates generated by a reliable HERS can be valuable information for those underwriting and insuring EEMs. Increased use of HERS for this purpose could help to speed the institutionalization of HERS and EEMs in the housing market.

Recognizing the potential for HERS and EEMs to stimulate home energy efficiency, the U.S. Department of Energy (DOE), in cooperation with the U.S. Department of Housing and Urban Development (HUD), initiated the National Collaborative on Home Energy Rating Systems and Mortgage Incentives for Energy Efficiency. The National Collaborative was created to prepare A Blueprint for Action, which is intended to extend the benefits of HERS and EEMs throughout the United States.

\footnotetext{
l"Energy efficiency," as used throughout this document, is intended to include both energy-efficiency measures (such as insulation and low-emissivity windows) and renewable energy technologies (such as passive solar design and solar domestic hot water systems), the use of which reduces a home's consumption of utility-supplied energy.
} 


\section{Background}

HERS and EEMs are not new. Numerous HERS ${ }^{2}$ and energy-efficiency certification programs ${ }^{3}$ have been started in the United States. For a variery of reasons, many have ceased to exist. Others have persevered and continue to operate with varying degrees of market penetration. They are sponsored $b y$ a number of different types of organizations with varyıng goals and program designs. Some examples follow.

- The Energy Rated Homes of America organization has programs in Alaska, Arkansas, Iowa. Rhode Island, Vermont, Virginia, and West Texas.

- Several states are beginning HERS programs, including Arizona, Californa, Colorado. Mississippi. Missouri, Nebraska, New York, and South Carolina.

- The cities of Fort Collins, Colo., and Austin, Tex., have rating programs.

- The "Good Cents" and "Super Good Cents" certification programs are supported by almost 300 utilities, especially in the South and the Pacific Northwest.

Energy-efficient mortgages have been available for more than 10 years. In the early 1980s, the five federal mortgage agencies and federally chartered financial institutions announced their willingness to buy, guarantee, or insure EEMs-Fannie Mae, Freddie Mac, FHA, DVA, and FmHA. ${ }^{+}$Appendix A gives a brief description of the existing EEMs programs. In a number of locales, HERS or energy certification programs have been approved as a means to access EEMs. In other areas, EEMs are sometimes used without a HERS basis.

However, despite the availability of both HERS and EEMs, the programs have been underutilized. Few buyers and lenders are aware of the EEM option. For lenders, it often is seen as representung additional paperwork. There is a lack of uniformity in the five different national EEM programs. Primary and secondary lenders are unsure of the reliability of the promised energy savings. There are limited data

\footnotetext{
${ }^{2}$ HERS measure and rate on a scale the relative energy efficiency of any house, regardless of age. efficiency, or fuel use. The rating is based on the thermal performance of the building envelope and the heating, ventilating, and air conditioning (HVAC) system and is obtained by an on-site inspection and calculations. HERS calculations include estimates of annual energy performance and costs and recommendations for cost-effective energy-efficiency improvements.
}

${ }^{3}$ A pass/fail home energy-efficiency rating system, or "certification program," is typically operated by utilities, home builders" organizations, or not-for-profit organizations. Energy- efficiency standards for these programs are developed using local building characteristics, construction practices, and climatic conditions. They usually include thermal envelope efficiency criteria and space conditioning efficiency criteria. Certification programs generally rely on a specified inspection/verification process to ensure rating consistency. Houses either pass or fail the inspection for energy efficiency.

${ }^{4}$ Federal National Mortgage Association, Federal Home Loan Mortgage Corporation, Federal Housing Administration, Department of Veterans Affairs, Farmers Home Administration. 
on the relationship between energy performance and housing values. And because homeowners are unaware of EEMs and do not inquire about them, this is perceived as a lack of market interest.

As a result, the potential national benefits of HERS and EEMs are not being realized. The henefits could include:

- An increase in the market penetration of energy-efficient new homes and energy improvements of existing homes

- A significant decrease in the estimated energy use by participating new and existing homes

- An increase in the number of families that could qualify as first-time homeowners by 250.000 each year ${ }^{5}$

- A significant reduction in environmental pollution.

Because of these potential benefits, HERS and EEMs have received the growing attenuon of the Administration and of Congress. The National Energy Strategy, issued in February 1991, states:

To encourage the more efficient use of mortgage financing for energy efficiency, the Departments of Energy and of Housing and Urban Development will increase financial and technical support to develop and encourage the voluntary acceptance of efficiency ratungs and their use in home financing. After at least 5 years of support for voluntary adoption. it will be required that information on energy efficiency and information on the available mortgage financing options be provided to home buyers prior to sale. ${ }^{6}$

Congress endorsed the use of energy-efficient mortgages in the National Affordable Housing Act of 1990, which directs HUD to develop a uniform plan to make housing more affordable through mortgage financing incentives for energy efficiency. HUD intends to draw on the recommendations of the National Collaborative in meeting that Congressional directive.

\section{The National Collaborative}

The National Collaborative's members represent 25 organizations and interests which, working together, can make HERS and EEMs a national reality. The members represent states, mortgage lenders, builders, remodelers, public and environmental interests, utilities, and existing HERS programs. For a list of National Collaborative members, see Appendix B.

The National Collaborative was supported by four Technical Advisory Committees (TACs) in the subject areas of EEMs, HERS, Implementation, and Awareness. The Collaborative Consensus Committee

\footnotetext{
${ }^{5}$ Joint Center for Housing Studies 1986.

${ }^{6}$ U.S. Department of Energy 1991/1992, page 11.
} 
(CCC) defined issues that it wanted the TACs to address. ${ }^{7}$ All of the technical issue papers prepared by these TACs, as well as special papers written by members of the CCC and others as part of the Collaborative process, are available in a separate volume, Going National with HERS and EEMs: Issues and Impacts, The Collected Papers of the National Collaborative. ${ }^{8}$

The first meeting of the National Collaborative was held March 26, 1991. Since then. Collaborative members have taken part in an intensive series of 10 meetings to prepare $A$ Blueprint for Action, plus numerous TAC and special subcommittee meetings and caucuses. ${ }^{9}$ The National Collaborative included participants with sharply different perspectives on some issues. Every statement included in $A$ Blueprint for Action required the concurrence of every individual participant in the Collaborative. Yet in only one area-the EEM program-members "agreed to disagree" on some of the provisions of a national program. But even here, there was a broad consensus on many actions that can be taken immediately while the parties work together to clarify some of the features of the national effort.

\section{A Blueprint for Action}

A Blueprint for Action is a compilation of the findings and recommendations of the National Collaborative regarding steps to be undertaken to establish a national EEMs/HERS program. With regard to EEM recommendations, a few areas of specific disagreement are clearly labeled as such.

The proposed "national program" consists of two main aspects: (1) revisions to current EEM programs with a goal of making them more user friendly, uniform, and substantive-and hence more effective; and (2) a national certification system to ensure the technical credibility of HERS programs to be used in conjunction with EEMs. A number of guidelines that local and state HERS programs would have to meet to attain certification as part of a voluntary national program are outlined. HERS programs not wishing to be part of the national EEM/HERS system would not be required to meet any of these guidelines.

This document consists of chapters on linking HERS and EEMs, characteristics of EEMs, characteristics of HERS, and implementation. The recommendations of the Collaborative regarding the linked EEMs/HERS program are described in Chapter 2. Chapter 3 characterizes an EEM program. It

\footnotetext{
${ }^{7}$ Appendix $\mathrm{C}$ lists the issues assigned to the four TACs by the Collaborative Consensus Committee (CCC). The TACs were responsible for providing technical recommendations to the $\mathrm{CCC}$; the $\mathrm{CCC}$ was responsible for the final technical decisions and policy formulations as they are reflected in $A$ Blueprint for Action. In this document, the term "National Collaborative" (or "Collaborative") is used to stand for the CCC.

${ }^{8}$ This report (NREL/TP-261-4706) is available through the National Renewable Energy Laboratory, 1617 Cole Blvd., Golden, CO 80401.

${ }^{9}$ Appendix D shows the meeting schedule.
} 
also presents characteristics of an EEM program presented by a number of nonlending members of the Collaborative, followed by the mortgage-lending representatives' response. Chapter 4 presents the consensus agreements on the characteristics that HERS programs would have to meet to be part of a national EEMs/HERS program. The document concludes with chapters on implementation strategies for linking EEMs and HERS, and the Collaborative's conclusions. Other supporting materials are included as appendixes.

A Blueprint for Action represents the final product of the first phase of discussions of the National Collaborative on these topics. Much further work is called for throughout the document, reflecting the view of the Collaborative that many specifics of a national program remain to be defined. 


\section{Chapter Two: Linking EEMs and HERS}

The goal of a national EEMs/HERS program is to promote energy efficiency in housing through a voluntary program that takes energy cost savings into account in the underwriting of mortgage loans. A national system will consist of compatible mortgage programs that are user friendly and readily available in the marketplace. These programs will rely on nationally consistent, technically credible HERS programs.

To achieve these goals, a national EEMs/HERS program must incorporate the following principles $^{10}$

1. It will apply to new and existing, energy-efficient and energy-inefficient housing.

2. The underwriting process will adequately recognize energy efficiency in making loans.

3. It will permit mortgage financing of energy-efficient construction and improvements.

4. It will indemnify lenders against added loss, if any, from borrower default.

5. It will provide a reliable technical basis to allow underwriting of morngage loans by providing the energy cost savings information to the lender.

This report examines EEMs/HERS issues discussed by the National Collaborative and possible ways of achieving the stated goals. The Collaborative has agreed upon a number of important issues. These agreements expedite the linkage of EEMs with HERS. They include the following:

1. Development of common standards among Fannie Mae, Freddie Mac, FHA, DVA, and FmHA. The mortgage-lending community will work to develop common standards, forms. and practices to make EEM programs more user friendly.

2. Removal of barriers. Each government agency and federally chartered financial institution will review its existing program to remove any unnecessary barriers and simplify the EEM process.

3. Sponsorship of educational, training, and promotional programs. Lenders, appraisers, realestate sales and marketing professionals, energy raters, and others will require training in using EEMS and HERS; consumer awareness of EEMS/HERS will need to be increased. Each government agency and federally chartered financial institution will promote the use of EEMs by increasing training and educational programs about them.

4. Collection of data. The agencies and federally chartered financial institutions will take steps to collect information on their EEM programs and provide the data to a single organization for further analysis and reporting.

5. Analysis of existing EEMs/HERS data to evaluate loss experience. In addition, the agencies and federally chartered financial institutions will take data that may currently exist concerning

\footnotetext{
${ }^{10}$ These are the themes that are more fully developed in the report.
} 
homes that have had an energy rating and been financed with an EEM, and cross-check those loans against the records of the loans they currently hold to generate current financial performance information.

6. Development of a nationwide HERS program. DOE, in concert with the HERS industry, will inituate the process to develop national guidelines for HERS programs that provide credibie information for the mortgage-lending process and other housing-market participants.

7. Assurance of quality control for HERS. The HERS community will help assure credibility hy developing and adopting effective quality-control programs governing software, personnel. data collection, labeling, and other components of HERS programs.

The National Collaborative recognizes that the EEM effor is in its formative stages. There is a great deal of data still to be collected and analyzed, and the risks are not fully quantified.

As noted earlier, Chapter 3 presents characteristics of a national EEM program and Chapter 4 presents characteristics of a national HERS program. A linked EEMs/HERS program is discussed further in the Implementation and Conclusion chapters. 


\section{Chapter Three: Energy-Efficient Mortgages}

\section{Characteristics of a Proposed EEM Program upon Which There Was Full Consensus}

An effective national EEM program requires that the major lending institutions establish a consistent and explicit mechanism for recognizing energy efficiency in the mortgaging process. After extensive analysis and discussion. the National Collaborative reached consensus on the following recommendations concerning the EEM program.

1. The EEM program should have the following general characteristics:

a. An EEM program should be available from all major lending and insuring agencies and for all regular types of mortgages.

b. An EEM program should be as uniform as practical among different types of mortgages and different lending and insuring agencies.

c. An EEM program should be simple to apply administratively. The forms, policies. procedures, rating systems, and so on should be uniform and as streamlined as possible.

d. Alternative approaches may be used, provided that they have characteristics that are at least equivalent to these provisions.

2. Lenders should be adequately indemnified for any additional risk assumed in financing the cost of energy-efficiency improvements.

3. An applicant for a loan to purchase or refinance a home that meets a nationally recognuzed voluntary consensus standard ${ }^{11}$ should be eligible for recognition of energy cost savings in the mortgage-qualifying process. Examples of such recognition could include increasing qualifying ratios $^{12}$ or adjusting one of the components used to calculate the ratios.

\footnotetext{
${ }^{11}$ There needs to be a nationally recognized consensus standard as a reference for the purpose of determining efficient versus inefficient housing and as a factor in calculating energy cost savings that can ne recognized in the EEMs. Additional work will be required to arrive at a reference and to assess its mpact with respect to mortgages. A distinction between "reference" and "baseline," for the purposes of clarity, is appropriate.
}

1. Reference is the nationally recognized consensus standard that determines energy efficient versus energy inefficient.

2. Baseline is the point from which energy cost savings are measured. For inefficient homes, when the intent is to make cost-effective energy-efficiency improvements, the baseline is the house as it actually exists. For efficient homes, the baseline will be an as yet undefined point that has an agreed-upon economic relationship to the reference.

${ }^{12}$ Qualifying ratios are percentage ratios that compare borrowers' anticipated monthly fixed housing expense and total monthly obligations to borrowers' stable monthly gross income for the purpose of evaluating the likelihood of meeting expenses involved in home ownership. 
4. A qualified applicant for a standard mortgage may qualify for an energy-efficient mortgage covering the cost of improvements that are determined to be cost effective by a nationally recognized HERS. The EEM program may include a maximum cost limit for allowed improvements. ${ }^{13}$

5 Underwriting procedures should recognize energy cost savings separately from compensating factors.

At the same time, lenders, real-estate sales and marketing professionals, and appraisers shoula work to raise public awareness of home energy efficiency and of related opportunities in the mortgagelending process. Also critical, of course, is that a nationally consistent, technically credible home energy rating system be made widely available.

\section{Consensus Agreements on EEM Issues and Recommended Actions}

Following are the consensus agreements on eight sets of recommended actions with respect 0 EEM-related issues.

Potential Risk. There may be an unquantified additional risk to the morgage-iending community ${ }^{14}$ from amortizing the full cost of energy improvements in mortgages. This is because:

1. Insufficient data have been collected on the performance of energy-efficient mortgages.

2. Current market conditions show that energy-efficiency items in a home have added little or no additional appraised value to the property. This may be because actual comparable market data for energy improvements may not be available.

3. Increased underwriting ratios and loan-to-value (LTV) ratios ${ }^{15}$ have been shown to increase borrower default risk in regular mortgages when considered by themselves.

\section{Recommended Actions for This Issue.}

i. For the short-run, government agencies and federally chartered financial institutions should review data that currently exist (to be obtained from organizations that currently rate homes and assist borrowers in obtaining EEMs) on mortgages that have been underwritten under their existing programs and determine whether such data are sufficient for a preliminary risk analysis to be performed. For the long-run, the mortgage-lending community should take steps to begin

\footnotetext{
1 The limitation on the amount only refers to this specific context and is not intended to limit amounts for other improvements or in other situations.

${ }^{14}$ The term "mortgage-lending community" is used throughout this document to refer to the government agencies and government-sponsored enterprises of FHA, DVA, FmHA, Fannie Mae, and Freddie Maci, as well as individual lenders.

${ }^{15} \mathrm{LTV}$ ratio is the relationship between the amount of the mortgage loan and the appraised value of the security, expressed as a percentage of the appraised value (see Glossary). 
collecting data on mortgages underwritten using their respective EEM programs and, as noted earlier, provide data to a single organization for further analysis.

If EEMs are to be implemented prior to the full availability of data, the mortgage-lending community should be indemnified for any additional risk from amortizing the full cost of energy improvements in mortgages. This could be accomplished through tax incentives, tax credits, or by a third party acceptable to the mortgage-lending community, should such sources become available. If the added borrower risk of EEMs is accepted as not being significantly greater than non-EEMs, the default rates for non-EEMs can be used as the anticipated default rate to quantify indemnification guarantees to the mortgage investor/guarantor. An analysis should be conducted to ascertain total risk, to identify methods of reducing risk, and to determine the extent to which indemnification is appropriate.

Cost of Energy-Efficiency Improvements. The additional cost of making a home energy efficient may prevent a borrower from qualifying for a mortgage.

\section{Recommended Actions for This Issue.}

1. The National Collaborative agreed that projected energy cost savings should be used as a separate factor in calculating whether a borrower qualifies for an EEM.

2. There should not be a limit on the cost of energy-efficiency improvements as long as market value appraisals or investor/guarantor indemnification and proper underwriting standards are used.

3. A HERS should be implemented to support a broad-based EEM system.

4. The existing EEM programs-described in Appendix A-should stay in effect until they are revised.

Handling Energy Cost Savings in Lending. There was disagreement about how the cost of energy improvements should be considered in mortgage financing. In some geographic areas, there may be limited market data on which to determine the estimated market value (through an appraisal) of energy-efficient housing. ${ }^{16}$ The mortgage-lending community has stated that the value of the property in lending decisions should be based on appraised market value.

Recommended Action for This Issue. The National Collaborative recognized the issue of whether to increase the mortgage amount for cost-effective energy improvements in the absence of comparable data to support an estimation of the market value of the property with those improvements. The mortgage-lending community representatives have agreed to consider credit enhancements in the form of acceptable third-party indemnification to the investor/guarantor to the extent that the mortgage amount is increased to include cost-effective energy-efficiency improvements not based on appraised market value.

\footnotetext{
${ }^{16}$ There are often insufficient data to determine if the market will recognize the value of energy efficiency in a dollar-for-dollar increase in property value.
} 
Increased Downpayment. Adding energy-efficient improvements to a home may increase the amount of the borrower's downpayment. For some buyers, the downpayment is the limiting factor in housing affordability. Loan underwriting standards require a minimum downpayment. If the cost of the home is increased by the cost of the energy improvements, in some cases the amount of the downpayment goes up proportionally. Borrower default risk is highly correlated with the amount of equity, and, thus. the commitment a borrower has to the home. Consequently, an EEM may require a higher downpayment.

Recommended Action for This Issue. One method of overcoming this barrier would be to allow acceptable third-party organizations to provide a grant to the borrower for the incremental downpayment. Grants may improve borrowers' chances to buy energy-efficient homes or to make energyefficiency improvements.

Mortgage Limits and Improvement Costs. Maximum mortgage limits make it difficult to add the cost of energy improvements to mortgages that are already at or near the limit.

Recommended Action for This Issue. Mortgage limits are set by legislation. The Collaborative recommended that current law be modified to increase the maximum mortgage limits for all energyefficient mortgages. (Freddie Mac and Fannie Mae cannot support having their loan limits changed: however, other groups could support this.) For example, HUD provides that for improvements involving renewable energy technologies, the maximum mortgage limit can be increased by up to $20 \%$.

Complexity and Delay in EEMs. Barriers and complexities prevent primary lenders, builders, real-estate sales and marketing professionals, remodelers, and home buyers from using the EEM process.

\section{Recommended Actions for This Issue.}

1. Additional incentives would be helpful.

2. All agencies will review their existing programs in an effort to remove any unnecessary barriers and to simplify their processes.

3. Common standards and uniformity are recommended. As noted earlier, the agencies and federally chartered financial institutions should develop common standards, forms, and practices to make their respective EEM programs more user friendly.

Training Needs. There is insufficient training for lenders, real-estate sales and marketing professionals, appraisers, and others in the process.

\section{Recommended Actions for This Issue.}

1. Each mortgage-lending agency and federally chartered financial institution should promote the use of EEMs by increasing training and educational programs.

2. The uniformity in standards, forms, and practices referenced above would assist lender training.

Credible HERS Programs. To qualify for consideration in an EEM, the home energy ratings should have credibility in the lending community. 
Recommended Action for This Issue. A nationwide HERS program with credible standards and effective quality-control mechanisms should be the basis for a nationwide EEM program.

\section{Specific Characteristics of an EEM Program Advanced by a Number of Nonlending Members}

With regard to the specifics of the EEM program, there remain some areas of uncertainty and some disagreements among Collaborative members over the most effective or most desirable course to take. It was generally agreed that there should be a balance between incorporating characteristics into the mortgage process that would effectively encourage energy efficiency and, at the same time, protect the mortgage-lending community from potential additional risk. However, the Collaborative was unable to agree on certain characteristics. In the interest of framing certain relevant points for future reference. the Collaborative agreed to present one model advanced by a number of nonlending members of the Collaborative. Section IV presents a brief discussion of the issues raised by the mortgage-lending members in response.

To these nonlending members, an EEM means a mortgage on a residential property that recognizes the energy cost savings of an energy-efficient home. Such an EEM qualifies borrowers who would otherwise have qualified for a mortgage on a similar home without cost-effective energy-saving construction or improvement features. Therefore, an EEM would remove the mortgage barriers and disincentives to the purchase of energy-efficient homes and to making energy-efficiency improvements in existing homes.

These nonlending members advanced the concept that an EEM program should implement certain characteristics in specific ways. The elements of an EEM program they focused on included loan qualification for energy-efficient and inefficient homes, calculating the maximum loan available, downpayment assistance, maximum mortgage limits, and supplemental mortgage insurance.

Loan qualification for energy-efficient homes. An energy-efficient home is one that has been built or improved to at least a nationally recognized voluntary consensus energy performance standard. ${ }^{17}$ For an energy-efficient home, the basic debt-to-income qualifying ratio should be increased by $2 \%$. For homes that exceed the standard, the added monthly cost savings should be subtracted from the normal housing costs (principal, interest, taxes, and insurance, or PITI) before calculating the qualifying ratio.

Loans for improvements to inefficient homes. For cost-effective ${ }^{18}$ energy-efficient improvements to homes that do not meet the nationally recognized performance standard, the energy cost

\footnotetext{
${ }^{17}$ The exact standard will be determined at a later date.

${ }^{18}$ The exact definition of "cost effective" will be determined at a later date.
} 
savings resulting from the improvements should, at the purchaser's election, be subtracted from the PITI. The loan's total amount should be increased by an amount equivalent to the present worth of the capitalization of the energy cost savings that will be realized.

Calculating maximum loan amount. Mortgages are generally limited to a percentage of the appraised value, for example, $90 \%$ or $95 \%$. For cost-effective improvements to inefficient homes, the mortgage amount should be increased by the total cost of the energy-saving improvements after the home's apprased value has been determined and the base loan has been approved. However, the increase should be limited to an amount that, when amortized along with the mortgage. can be paid for by the monthly energy cost savings. And the money for the energy improvements should be put into escrow with the title company or other depository at closing.

For energy-efficient homes, conceptually the mortgage amount should be increased as described above. However, on new homes, this is difficult to compute. Therefore, the mortgage amount should be increased by an amount up to $5 \%$ of the home's appraised value.

Downpayment assistance. Federal, state. or other public or private programs should develop programs to provide grants to home buyers to cover the additional downpayment for the incrementat increase in the loan amounts described above. Private groups such as utility companies and home builder associations may be interested in establishing such programs. Lending institutions should accept the grant money as if it were the borrower's own funds.

Maximum mortgage limits. A mortgage on an energy-efficient home should be allowed to exceed the maximum mortgage limit in effect at the time by up to $5 \%$.

Supplemental mortgage insurance. To minimize any added risk of default to lenders for the added mortgage value of EEMs, the amount added to the mortgage for energy-efficiency features should be covered with supplementary mortgage insurance for five years.

\section{The Mortgage-Lending Members Respond}

The members of the National Collaborative who represented the mortgage-lending community (those involved in determining how credit is granted in the secondary mortgage market, which provided more than $\$ 453$ billion in mortgage financing in 1990) did not completely endorse or support the preceding suggested characteristics of an EEMs program (Section III).

As the primary risk takers in the real-estate finance process, the mortgage-lending members believed the adoption of certain of the suggested characteristics could expose the mortgage-lending community to additional risks and losses that are currently unknown and unquantified. This was unacceptable to those charged with maintaining the stability of the national mortgage market.

Within the spirit of the National Collaborative process, the mortgage-lending community members respected and appreciated the views expressed by those members of the National Collaborative who did 
not share in the mortgage risks associated with EEMs. However, it is important that concerns related to issues identified during the National Collaborative process be defined as those on which the National Collaborative did not reach consensus. These issues follow:

Property valuation. The value of a property used in the mortgage underwriting process must be based on the property's market value. Any increase in the value of a property due to an improvement (including energy-efficiency improvements) must be based on the market's response to the improvement itself-not its cost. Deviation from this fundamental appraisal approach to valuation exposes the mortgage-lending community to an additional risk of greater loss if the market does not recognize the entire cost of an improvement as a dollar-for-dollar increase in the property value.

Borrower qualification-income and expense considerations. The possibility that a borrower will experience lower energy costs in an energy-efficient house than in an energy-inefficient house is a factor that can be considered in the mortgage underwriting process. The extent to which a uniform, nationally recognized HERS will help estimate an average amount of expected energy cost savings for a specific house helps to estimate the possible impact for a given borrower. However. the possibility of lower energy costs is only one of the many factors that need to be evaluated in the mortgage underwriting process. Energy costs are not a fixed expense like the mortgage payment itself, property taxes, and insurance. In fact, energy costs vary greatly, depending on climate zone, weather conditions. and individual lifestyles.

The mortgage-lending community cannot accept the unknown level of default risk imposed by any formula approach to income qualification that assumes a theoretical reduction in fixed monthly mortgage payments based on an estimation of possible energy cost savings by the occupantborrower. Such an approach would elevate the perception of possible energy cost savings to a level of significance in the mortgage underwriting process for which no empirical basis currently exists.

The mortgage-lending community supported the concept that the estimated encrgy cost savings may be used as an additional factor in calculating whether a borrower qualifies for a specific mortgage, rather than the use of some formula or factor that has no relationship to the actual energy costs and/or savings of a specific house. ${ }^{19}$

Supplemental mortgage insurance. For conventional mortgage loans, the use of supplemental mortgage insurance to cover any additional loss due to default may be unworkable for several reasons. First, although the use of insurance transfers the risk to a private mortgage insurance company, the lender must rely on the private mortgage insurer's ability to pay a claim. By adding more risk to the insurer's portfolio in the form of the supplemental insurance, the financial stability of the insurer is weakened. This could place the lender at greater risk. Second, the supplemental mortgage insurance

\footnotetext{
${ }^{19}$ It should be noted that HERS rate specific houses.
} 
coverage will increase the borrower's cost of obtaining a mortgage. This cost may need to be compared to the estimated energy cost savings for the home. Third, the insurance industry is regulated at the state level. Many state regulations prohibit mortgage insurers from issuing policies where the loan-to-value ratio exceeds $95 \%$.

If viewed individually. the suggested EEM characteristics may appear to be minor changes to mortgage program users and beneficiaries. However, when the suggested EEM characteristics are viewed in total. and within the context of the whole national mortgage market and its needs, they become unacceptable to the mortgage risk takers.

Each member of the mortgage-lending community must analyze and maintain EEM programs within the context of their respective roles and morgage programs, while not losing sight of the needs of the national mortgage market. The members of the mortgage-lending community must balance the risks associated with the origination, purchasing, insuring, and guaranteeing of all mortgages, including EEMs. with the need to maintain financial stability and ensure the continuing availability of funds for the national mortgage market.

The mortgage-lending community will continue to work toward a uniform national program that will promote energy efficiency in housing. As progressive steps are taken to achieve this goal. the risks and losses will be evaluated. By taking incremental advances based on appropriate risk assessment, the nation's mortgage industry will remain sound in order to continue to offer mortgage financing opportunities. 


\section{Chapter Four: Home Energy Rating Systems}

A significant number of HERS have been established since 1980. Some have ceased to exist: others continue to operate. All have faced significant market obstacles. ${ }^{20}$

To a large degree, HERS in different locales have developed independently. This has resulted in many unique programs. Because no single HERS model exists, new programs continue to expend significant resources on research and development. The local variation in the programs makes it impossible to build a universal link between HERS and a national EEM program. Further, most HERS use different calculational approaches, or "tools." with little or no documentation of technical accuracy. In fact, technical standards for HERS have never existed. Lenders and others have questioned the reliability of energy-use and savings estimates generated by HERS.

A primary focus of the National Collaborative's HERS deliberations was to define a set of guidelines that would address the concerns of technical credibility. These guidelines deal with the basic design and operation of HERS programs and set accuracy standards that "certified" HERS programs must meet if they are to participate in the national program. The guidelines are also designed to promote uniformity among different HERS to facilitate a uniform link to a national EEMs/HERS program.

At the same time, care was taken to specify minimum features to allow local programs substantial flexibility to meet local needs. This approach recognized that the national effort should build upon existing local HERS and bring them into a national framework, or umbrella, rather than strive to create a single. nationally administered HERS.

The recommended guidelines are largely based on the desire for a EEMs/HERS link; however. they will also provide useful guidance for independent HERS programs. Only programs that wish to be certified under the national EEMs/HERS program would have to meet these guidelines.

The consensus findings of the National Collaborative follow. ${ }^{21}$

\section{Design and Operation of HERS}

The design and use of HERS tools should follow these principles.

${ }^{20} \mathrm{Cf}$. Vories and George 1991.

${ }^{21}$ The HERS TAC was notable among the Collaborative's four TACs in that it achieved a consensus recommendation on each of the 21 issues it was assigned. While not all of these recommendations were adopted by the $\mathrm{CCC}$, they provide a useful guide to state-of-the-art technical thinking on HERS. As noted elsewhere, the full set of papers is available in Going National with HERS and EEMs: Issues and Impacts, The Collected Papers of the National Collaborative. 
Basic measure on which ratings should be based. The basic unit of measure should be total annual energy use in Btu/year by fuel type. The HERS calculation tool should be capable of converting these quantities to all commonly used normalization parameters.

Fuel neutrality. Fuel neutrality will be addressed by requiring verified HERS calculation tools to produce accurate energy-use and cost projections. Other issues relating to energy rype should he decided at the state or local level.

Treatment of occupant behavior. To ensure consistency and credibility, a HERS calculation tool should rate the home using standardized occupancy assumptions. Those features of the home under control of the occupants-such as thermostat set points, removable lights and appliances, whole-house fans. and water-heater set points-will be assumed to be fixed in the rating calculation tool to account for occupant-related factors. The HERS calculation tool should incorporate average estimates for lifestyle parameters to account for average occupant behavior. The average estimates need to be as locationspecific as possible, and they need to be applied consistently.

Differentiation between new and existing housing. New and existing homes should not be differentiated, from a technical standpoint, in the calculation of energy use by HERS calculation procedures. In practice, specific HERS programs may offer different services with respect to new and existing homes.

Building types. HERS should be applicabie to multifamily, single-family, and manufacrured housing. A HERS calculation tool should be certified or verified for those building types it is intended to rate.

Home features measured by a HERS. A HERS calculation tool should provide the estimated annual energy performance of the building in Btu/year and dollars for fuel type. That number is composed of the energy use associated with the performance of the following:

- Thermal envelope

- Mechanical heating, ventilating, and air conditioning (HVAC) system

- External load controls ${ }^{22}$

- Hot water service

- Optional features, including hard-wired lights, major appliances and controls, and energystorage systems.

Specific recommended features to be included in HERS calculation tools remain to be defined.

Inclusion of innovative features. The certifying process for HERS tools would allow qualification according to the building types, features, and climate zones for which a local HERS

\footnotetext{
${ }^{22}$ External load controls are fixed or movable shading elements (e.g., awnings, wing walls, overhangs, eaves, and shade screens) that control solar heat gain to exterior envelope components.
} 
calculation tool is intended. Certified HERS should also provide for alternative rating calculation tools when a simplified input tool cannot handle particular buildings, energy fearures, or new technologies. HERS should strive to keep pace with new technological developments by periodically upgrading its calculational tools.

\section{Accuracy of HERS Output}

All HERS must be capable of producing consistent and accurate ratings. There are two levels of accuracy. The first is the accuracy of the HERS calculation tool. The second is the accuracy of the entire delivered home energy rating system, including the energy inspection, the translation of energy-inspection information to input data, the recommended cost-effective energy-efficiency improvements, the standardized occupant and operating assumptions, field inspections, and the use of the HERS calculation tool to produce an energy and dollar estimate.

Accuracy of the HERS calculation tool. A HERS calculation tool should accurately project energy use in energy units and dollars. To be certified as an approved HERS calculation tooi. calculational accuracy within a prescribed range relative to benchmark results from state-of-the-art detailed simulation models must be demonstrated (exact procedure to be developed). The acceptable range should he determined, recognizing the trade-off between accuracy, ease of use, cost of performing the rating, and the needs of the EEM.

Accuracy of the entire HERS system. To maintain certification under the national HERS umbrella. HERS would be required to collect data, such as short-term data, long-term submetering data. or utility bill data to verify overall quality control of the system and to allow periodic self-correction (data collection protocols to be developed).

Rater training and quality control. Rater training and qualification should include a mix of classroom and on-site training, concluding with certification by testing and demonstration of proficiency. HERS programs are responsible for quality control of their raters.

\section{Other Recommendations for HERS}

The National Collaborative made four other recommendations concerning a national HERS program.

HERS procedures for retrofits and renovations. For a HERS to qualify for the national program, its calculation tool's affiliated savings estimates need to take into account the potential interactive effect of the energy features on energy and cost savings.

Procedures for rating groups of new buildings. Building ratings should be permitted to be grouped when technically feasible, when quality control can be demonstrated, and when accuracy criteria will be met. Group ratings will reduce duplication of effort and cost and will provide a common 
basis for comparing homes. Group ratings are most appropriate when applied to the same home design built multiple times on comparable sites, or when applied consistently within the criteria of a certification program. Homes can be spot-checked to verify the accuracy of the rating; each home would not have to be individually rated.

Allowance of different rating approaches. A single rating method and scale should be the basis for linking EEMs and HERS as an element of the national program. A "rating method," as used here. is the equation and associated inputs that describe the relationship of a rating to an amount of energy use. energy cost, or other measure of efficiency. Local systems should be able to use any designation they choose to present rating information to users within their service area. A single rating method would facilitate linkage to a uniform national EEMs/HERS program. Further study is recommended to determine the form, parameters, and inputs of the rating method.

Requirements for re-rating of homes. Homes for which EEM financing is desired may need to be re-rated at the time of sale or refinancing if the lenders so require. If the rated home's features remain unchanged and the rating scale for that locale remains the same, the home may not need to be rerated.

National data base. Lack of data is the largest hurdle to setting up a successful national EEMs/HERS program. Each HERS program in each jurisdiction should keep accurate records of every house rated. There needs to be a national data base on energy-rated homes and EEM homes. A national program should develop a set of guidelines for minimum requirements for state and local data bases to allow data retrieval and reporting to the national data base. 


\section{Chapter Five: Implementation}

During 1991, the National Collaborative was able to accomplish a great deal; yet much more remains to be done to fully develop. promote, and implement a national EEMs/HERS program.

For some of the following items, participant groups can take action on their own to make further progress. Most items, however, will require a more organized effort. DOE, in collaboration with HUD. should take the initiative to prioritize the following tasks and take appropriate actions to make progress in each of the listed areas. These actions could include, among other things, public meetings. conferences. appointment of one or more implementation task groups, and the retention of contractors.

Included among the unfinished business is the following:

- Distribution of $A$ Blueprint for Action for public comment

- Revision of $A$ Blueprint for Action based upon the substance of the public comment

- Further development of mechanisms for program implementation, including mechanisms to develop and manage the following:

- Technical clearinghouse

- Information cleannghouse

- HERS accreditation system

- Rater credentialing system

- Data collection system

- Data analysis program

- Quality assurance program

- A business plan

- A public information and education program

- Labeling criteria

- The HERS rating method

- A nationally recognized consensus standard to define energy efficiency

- An adequate indemnification mechanism

- Demonstration programs

- Common standards, forms, and practices by the agencies and enterprises.

A report summarizing progress made during 1992 should be prepared at the end of 1992 . 


\section{Chapter Six: Conclusion}

The National Collaborative, through a consensus-building process, has defined concepts for linking home financing to a successful national program of home energy rating systems. Substantial progress has been made in educating all National Collaborative members on needs of both the energy rating and the home financing industries. Most importantly, a momentum has evolved that has the potential for results and ultimate realization of the National Collaborative's goals.

A responsibility of the members of the National Collaborative is to sustain this momentum, further integrate interests and viewpoints, and foster continued constructive communications among key interest groups. A strategy for meeting this responsibility is to sponsor demonstration and field testing of certain assumptions and program goals outlined in A Blueprint for Action. These tests would be carefully planned and focused, highly controlled, and designed to maximize input to the national program and provide relevant data to the future deliberations of the mortgage-lending community and for further program development.

The secondary mortgage-market agencies should work directly with local HERS/EEMs program administrators in a limited pilot program to test the proposed EEM program that emerges from the National Collaborative. Elements of such a pilot program could include, but would not be limited to:

- Elements designed to encourage the program's use and overcome hurdles (incentives could be one of these elements)

- Guidelines to allow ease of financing energy-efficient improvement and construction features and recognition of energy-efficient properties, while at the same time, indemnifying lenders from risk

- Sponsorship of educational, training, and promotional programs in coordination with the local HERS/EEMs administrator

- A built-in mechanism to allow mid-course corrections for program fine-tuning

- An established goal for the pilot program against which to measure success

- Collection of data relevant to the evaluation of the pilot program.

The National Collaborative hopes that the momentum initiated during its 1991 deliberations will result in numerous organizations effectively carrying forward the development of HERS linked to a national program of amortizing the cost of energy improvements in housing through mortgage financing. 


\section{References}

Joint Center for Housing Studies of the Massachusetts Institute of Technology and Harvard University. May 1986. The Effect of the Shelter Industry Energy Rated Homes ${ }^{T M}$ System on Homeownership. Boston, MA: Joint Center for Housing Studies of the MIT and Harvard University.

National Collahorative. 1992. Going National with HERS and EEMs: Issues and Impacts. The Collectea Papers of the Nattonal Collaborative. (NREL/TP-261-4706) Golden, CO: Nauonal Renewable Energy Laboratory.

U.S. Department of Energy. 1991/1992. National Energy Strategy: Powerful Ideas for America. Washington, D.C.

Vories, R. and K. George. 1991. Analysis of Home Energy Rating Systems: Results of Brief Intentews. Denver, CO: Infinite Energy. 


\section{Appendixes}




\section{Appendix A: Existing Energy-Efficient Mortgage Programs}

\section{Introduction}

Energy bills are usually the largest portion of housing costs after rent or mortgage payments, averaging about $\$ 1.100$ a year - fften twice that much in severe climates. For lower-income households, energy costs represent a proportionately greater fraction of the budget than for more affluent households Although the energy efficiency of a typical residence could be improved. buyers of new or existing homes may not be able to afford the additional cost of energy improvements without mortgage incentives.

The energy-efficient morgage (EEM) reduces homeownership costs by enabling homeowners to achieve meanngful energy savings with modest increases in mortgage payments. It allows the borrower to add the cost of energy measures to the mortgage, thereby providing the longer term, lower interest rate, and tax benefit of mortgage financing. The small increase in mortgage payments is typically more than offset by energy savings. In general, the EEM concept takes into account both the incremental capital cost and the reduced operating cost of an energy-efficient home.

Homes that are already energy efficient. An EEM allows more lenient rules in qualifying borrowers for mortgages on homes that meet certain levels of energy efficiency. Because the efficient home has lower energy bills, the borrower can spend a larger-than-normal portion of income on the basic mortgage payment. Although often applied to new construction and promoted through builders and their lenders. this EEM option is also available for existing energy-efficient homes.

Homes that require energy efficiency improvements. The other EEM option applies to the purchase or refinancing of existing homes that need energy upgrades.

In either case, the home becomes more affordable, as well as more comfortable and more valuable, with henefits to the buyers, seller, and lender. The EEM is a WIN-WIN-WIN concept.

\section{EEM Programs Available}

Several EEM programs have been implemented since 1980. National programs are available through three federal government organizations involved in home financing. In addition, the two major secondary mortgage lenders also have developed EEM programs.

All the EEM programs include guidelines to determine (a) whether the home meets the requisite level of energy efficiency, (b) which energy improvements may be added into the mortgage, and (c) how the limits on borrower qualification may be broadened to take reduced energy costs into account.

- The Federal Housing Administration (FHA) of the Department of Housing and Urban Development (HUD) insures home mortgages for qualifying home buyers. HUD/FHA allows an increase in the loan amount within certain limits and "stretches" the income qualifying ratios for mortgages on new homes meeting specific guidelines for energy efficiency.

- The Department of Veterans Affairs (DVA) guarantees mortgages for qualifying veterans with very little or no down payment, with the maximum loan amount determined by the participating lender. DVA allows an increase in the mortgage amount for energy-related improvements, within cerrain limits and with appropriate documentation.

"This material was prepared by the Office of Building Technologies, U.S. Department of Energy, November 1991. 
- The Farmers Home Administration (FmHA) does not have a specific category of energyefficient mortgages, but instead requires conformance with its thermal performance construction standards for all its loans, which are provided directly to qualifying borrowers in rural areas (under 10,000 population). FmHA will loan an additional amount for improvements, including those to increase energy efficiency, that are economically justified in accordance with "value-in-use" techniques.

- The Federal National Mortgage Association (FNMA or "Fannie Mae") and the Federal Home Loan Mortgage Corporation (FHLMC or "Freddie Mac") have developed criteria for home mortgages they are willing to buy and have promulgated these rules to their approved mortgage seller/servicer institutions, who in turn offer such loans to borrowers. Both encourage energy efficiency by providing specific criteria for EEMs they are willing to buy on the secondary mortgage market.

\section{Getting Started: Applying for an EEM}

An application for an energy-efficient mortgage should be directed to a local mortgage lender who participates in the EEM programs of a secondary mortgage lender (such as Fannie Mae or Freddie Mac) or an agency insuring or guaranteeing the mortgage. such as HUD/FHA or DVA, if applicable. A borrower does not apply through these organizations directly.

Because lenders process mortgages according to well-established procedures, timing is critical. Therefore, a borrower wishing to obtain an EEM should discuss this with the lender early in the mortgage application process. While shopping for a home, the borrower should identify a participating EEM lender and find out its specific requirements. If the home already meets an accepted energy efficiency standard. the purchaser should provide the documentation to the lender as soon as possible in the mortgage application process. A borrower hoping to finance energy conservation improvements as part of the mortgage will need to have the planned upgrades documented by an appraiser or energy consultant, as the lender requires, and installation will have to be completed within a required period of time.

\section{Sources of Information on Energy-Efficient Mortgages}

These sources are especially helpful for borrowers and lenders.

HUD/FHA: The booklet Guide to Single Family Home Mortgage Insurance (HUD-1235-H(2), April 1990), gives brief descriptions of the various HUD/FHA programs. HUD/FHA Mortgagee Letter 89-25 (October 20, 1989) states the HUD/FHA guidelines for approval of EEMs.

Contact the HUD Field Office nearest you, or the U.S. Department of Housing and Urban Development, Assistant Secretary for Housing/Federal Housing Commissioner, Washington, DC 20410-8000.

Department of Veterans Affairs: VA-Guaranteed Home Loans for Veterans (VA Pamphlet 26-4, August 1989), summarizes the eligibility criteria and application steps for VA loans in general. DVB Circular 26-80-31 (August 8, 1980; Change 3, December 10, 1985), Loans to Purchase Homes and Make Energy Conservation Improvements, states the modification of VA property valuation and loan processing procedures for including energy conservation improvements in loans for existing homes.

Contact the Loan Guaranty Division of the VA Regional Office in your state; these offices are listed in the pamphlet cited above.

Farmers Home Administration: Information on the FmHA loan program is available directly from the 2,200 local county and district FmHA offices, generally located in county seats of rural areas. 
Federal National Mortgage Association (Fannie Mae): A brochure, Energy Conservation Home Financing (January 1990), briefly describes the Fannie Mae EEM program. Product Highlights of August 1990, "Energy Conservation Home Financing," summarizes the EEM provisions contained in various sections of Fannie Mae's Selling Guide for approved lenders.

Contact the Fannie Mae Home Office, 3900 Wisconsin Avenue, NW, Washington. DC 20016-2899. or one of the five Regional Offices listed in Product Highlights.

Federal Home Loan Mortgage Corporation (Freddie Mac): Prospective borrowers should contact their local lender about Freddie Mac's EEM program.

For mortgage lenders, see Freddie Mac's Industry Letter, "Energy-efficient mortgage program and revised energy addendum" (July 21, 1989), which outlines the EEM program, including references to sections in Freddie Mac's Sellers and Servicers Guide For a copy, contact your Freddie Mac marketing representative or Freddie Mac, 8200 Jones Branch Drive. McLean, VA 22102.

For more information: Contact NATAS, P. O. Box 2525. Butte MT 59702

$1-800-428-2525$

1-800-428-1718 (in Montana) 


\section{Appendix B: Members of the National Collaborative}

Listed are members of the Collaborative Consensus Committee (CCC), members of the collaborative technical advisory committees. members of the CCC's Steering Committee, staff, and officiai observers.

\section{Collaborative Consensus Committee (CCC) Members}

Alliance to Save Energy (ASE) - Bill Prindle (Member), Washington. DC

American Association for Reured Persons (AARP) - George Gaberlavage (Member), Washington. DC: Jo Reed (Alternate), Washington, DC

American Gas Association (AGA) - Alan Hudenets (Member), Arlington, VA; Mary Carson (Alternate). Arlington. VA

American Public Power Association (APPA) - Barry Moline (Member), Washington. DC; Doug Swartz (Alternate), Fort Collins, CO

American Society of Home Inspectors (ASHI) - Michael Lennon (Member), Falls Church. VA

Appraisal Institute (AI) - Steve Azia (Member), Washington, DC

Association of Energy Efficient Mortgage Service Companies (AEEMSC) - Jim Curtis (Member), Palo Alto, CA

Califomia Home Energy Rating System, Inc. (CHERS) - Jim Fulkerson (Member), San Francisco. CA Consumer Federation of America (CFA) - David Swankin (Member), Washington, DC; Rebecca Cohen (Alternate). Washington. DC

Edison Electric Institute (EEI) - Tom Farkas (Member), Washington, DC

Energy Efficient Builders Association (EEBA) - Don Buchan (Member), Ottawa, Ontario, Canada

Energy Rated Homes of America (ERHA) - Ron Hughes (Member), Little Rock, AR

Farmers Home Administration (FmHA) - Rich Davis (Member), Washington, DC

Federal Home Loan Mortgage Corporation (Freddie Mac) - Charles Fritts (Member), Washington. DC: Jane Stockinger (Alternate), McLean, VA

Federai National Mortgage Association (FNMA - Fannie Mae) - Chip Coffay (Member), Washington, DC

Federal Housing Administration (FHA) - Mark Holman (Co-Member), Washington, DC; Morris "Bud" Carter. (Co-Member), Washington, DC

Federal Institutions Examination Council, Appraisal Subcommittee - Ted Baker (Member), Washington, DC

Mongage Bankers Association (MBA) - Bill Brewster (Member), Washington, DC

National Association of Home Builders (NAHB) - Perry Bigelow (Co-Member), Palatine, IL; Charles Field (Co-Member), Washington, DC

National Association of the Remodeling Industry (NARI) - Patti Knoff-Burgio (Member), Arlington, VA

National Association of REALTORSTM (NAR), Govemment Relations Division - Lee White (Member),

Washington, DC; George Griffin (Alternate), Washington. DC

National Association of State Energy Officials (NASEO) - Steve Baden (Co-member), Anchorage, AK:

Doug Culbreth (Co-member), Raleigh, NC; Chris Roitsch (Co-member), Austin, TX

Natural Resources Defense Council (NRDC) - Dan Lashof (Member), Washington, DC; Marika Tatsutani (Alternate), Washington, DC

Southern Electric International, Good Cents Division - Benny Folsom (Member), Atlanta, GA

U.S. Department of Veterans Affairs (DVA) - Robert Finneran (Member), Springfield, VA 


\section{Technical Advisory Committee (TAC) Members}

\section{Home Energy Rating Systems (HERS) TAC}

CCC Co-Chair: Bill Prindle, Alliance to Save Energy, Washington, DC (now with Barakat and Chamberlin)

CCC Vice Chair: $\quad$ Steve Baden, National Association of State Energy Officials, Anchorage, AK

Staff Co-Chair: Ron Judkoff, National Renewable Energy Laboratory, Golden, CO

Members:

Tim Butler, Energy Division, North Carolina Department of Economic and Community Development, Raleigh, NC

William Freeborne, Policy Development and Research, U.S. Department of Housing and Urban Development, Washington, DC

Michael Holtz, Architectural Energy Corporation, Boulder, CO

Bion Howard, Alliance to Save Energy, Washington, DC

Steve Kennedy, Georgia Power, Atlanta, GA

Ron Nickson, National Association of Home Builders, Washington, DC

Doug Swartz, Light and Power Utility, City of Fort Collins (Co-member with IMP TAC), Fort Collins, CO

Steve Szoke, Passive Solar Industries Council, Herndon, VA

\section{Energy-Efficient Mortgages (EEMs) TAC}

CCC Co-Chair: Bill Brewster, Mortgage Bankers Association, Washington, DC

Staff Co-Chair: $\quad$ Ken Crandall, Federal Housing Administration, Washington, DC

Members:

Richard Faesy, Energy Rated Homes of Vermont, Burlington, VT

Matt Felber, Farmers Home Administration, Washington, DC

Walter Patterson, Texas Energy Extension Service, Arlington, TX

William Sorrentino, National Association of Home Builders, Washington, DC

Jane Stockinger, Federal Home Loan Mortgage Corporation (Freddie Mac), McLean, VA

\section{Implementation TAC}

CCC Co-Chair: Charles Field, National Association of Home Builders, Washington, DC

Staff Co-Chair: $\quad$ Rebecca Cohen, Consumer Federation of America, Washington, DC

Members:

David Dacquisto, National Association of Home Builders, National Research Center, Upper Marlboro, MD

Russell Duncan, Carolina Power and Light, Raleigh, NC

Javier Figueroa, Washington State Energy Office, Olympia, WA

Stuart Harshbarger, Washington Gas Light,

Doug Seiter, City of Austin, Austin, TX

Doug Swartz, Light and Power Utility, City of Fort Collins (Co-member with HERS TAC), Fort Collins, $\mathrm{CO}$

Richard Tracey, Ryland Homes, Columbia, MD 


\section{Awareness TAC}

CCC Co-Chair:

David Swankin, Consumer Federation of America, Washington, DC

Staff Co-Chair:

Gerry McGowan. Philadelphia Department of Energy Support Office. Philadelphia. PA

Members:

William Griffin, Commonwealth Edison. Chicago, IL

Dehorah Howard. Anzona Energy Office, Tucson, AZ

Randy Martun. Iowa Department of Natural Resources, Des Moines, IA

Rebecca Vories, Infinite Energy, Denver, CO

Staff

U.S. Department of Energy (DOE)

John P. Millhone. Deputy Assistant Secretary, Office of Building Technologies, Washington, DC Mary-Margaret Jenior, Program Manager, Office of Building Technologies, Washington. DC Gerry McGowan, DOE Philadelphia Support Office. Philadelphia. PA

National Renewable Energy Laboratory (NREL)

Barbara C. Farhar, HERS/EEMs Program Leader, Collaborative Co-Facilitator. Wasinngton. DC Ron Judkoff. Staff Co-Chair, HERS TAC, Golden, CO

Dorian McDuffie. Alyssa Tonelli, Kim Rattley, Conference Arrangements, Washington, DC

Lauren Raphael, Technical Writer, Washington, DC

Mary Anne Dunlap, Ann Brennan, Technical Editors. Golden, CO

Kay Vernon, Janet Fried, Word Processing, Golden, CO

Princeton Economic Research, Inc. (PERI)

Joseph M. Cohen. Minutes. Rockville, MD

L.S. Deparment of Housing and Urban Development (HUD)

Energy Division: Bob Groberg, Lois Dean, Observers. Washington, DC

Federal Housing Administration (FHA): Ken Crandall, Staff Co-Chair EEMs TAC, Washington. DC

Facilitator: John A.S. McGlennon, ERM-New England. Inc., Boston, MA

\section{Steering Committee Members}

Steve Baden, National Association of State Energy Officials, Anchorage, AK

Bill Brewster, Mortgage Bankers Association. Washington, DC

Rebecca Cohen, Consumer Federation of America, Washington, DC

Ken Crandall, Federal Housing Administration, Washington, DC

Barbara Farhar, National Renewable Energy Laboratory, Washington, DC

Mary-Margaret Jenior, U.S. Department of Energy, Washington, DC

Ron Judkoff. National Renewable Energy Laboratory, Golden, $C O$

Gerry McGowan. U.S. Department of Energy, Philadelphia Support Office, Philadelphia, PA

John Millhone, U.S. Department of Energy, Washington, DC

Bill Prindle, Alliance to Save Energy, Washington, DC

David Swankin, Consumer Federation of America, Washington, DC 


\section{Official Observers}

American Gas Association - Paul Cabot, Jane Marder, Bruce Savage, Arlington, VA

Appraisal Foundation - Deborah Sharp, Washington, DC

Appraisal Institute - Don Kelly, Washington, DC

Arizona Energy Office - Charles Gohman, Phoenix, AZ

Air Conditioning and Refrigeration Institute - Richard Denny, Arlington, VA

California Energy Commission - John Wilson, Sacramento, CA

City of Manassas - Randy Bowers, Manassas, VA

Communications Consulting - Laura Braustein, Reston, VA

Council of American Building Officials - Bob Spangler, Falls Church, VA

Electric Power Research Institute - Whitney Carroll, Michael Tinkleman, Washington, DC

Energy Efficient Builders Association - Bill Eich, Spirit Lake, IA

Energy Mines and Resources-Canada - Jack Cole, Ottawa, Ontario, Canada

Energy Rated Homes of America - Evan Brown, Dana Nixon, Little Rock, AR

Environmental Protection Agency - Adam Klinger, Washington, DC

Federal Home Loan Mortgage Corporation - Virgil Griffin, Washington, DC

Federal National Mortgage Association - John Nevin, Mark Simpson, Washington, DC

Florida Energy Office - Daryl O'Connor, Tallahassee, FL

Home Automation Association - Michael Coffey, Eric Davidson, Nick Pyle, Washington, DC

Independent Bankers Association - Marti Sworobuk, Washington, DC

Mineral Insulation Manufacturers Association - George Phelps, Alexandria, VA

Maryland Building Industries Association - Frank Merditch, Landover, MD

Mortgage Insurance Companies of America - Frank Balser, Washington, DC

National Association of REALTORSTM - Deborah Lassman, Steve O'Connor, Washington, DC

National Association of Regulatory Utility Commissioners - Michael Foley, Washington, DC

National Institute of Standards and Technology, Heat Transfer Group - Fanney Hunter, Bob Zarr,

Gaithersburg, MD

National Association of State Energy Officials - Frank Bishop, Washington, DC

National Energy Specialist Association - Frank Newbraugh, Topeka, KS

National Renewable Energy Laboratory - Ren Anderson, Golden, CO

Natural Resources Defense Council - Jennifer Jordan, Washington, DC

New York State Energy Office - David Abrey, Kevin O'Brien, Albany, NY

Pennsylvania Electric - Steve McDonnell, Montrose, PA

RISE - Deborah Curry, Providence, RI

The Society of the Plastics Industry, Inc. - George H. Sievert, Washington, DC

Southern California Edison - Gregg Ander, Rosemead, CA

U.S. Department of Energy - Ted Kapus, Sarah Kirchen, Simon Sidamon-Eristoff, Washington, DC

U.S. Department of Energy, New York Support Office - Joy Cazeaux, New York, NY

U.S. Department of Housing and Urban Development - Andrew Euston, Dick Manuel, Heidi Martin,

Roxanne Zimmerman, Washington, DC

U.S. Department of Veterans Affairs - Doug Widener, Washington, DC

U.S. Environmental Protection Agency - Adam Klinger, Washington, DC

U.S. League of Savings Institutions - Danielle French, Washington, DC

Vermont Housing Finance Agency - Doug Lothrop, Burlington, VT

Virginia Energy Office - Jim Smith, Richmond, VA 


\begin{tabular}{|c|c|c|c|}
\hline $\begin{array}{l}\text { Document Control } \\
\text { Page }\end{array}$ & $\begin{array}{l}\text { 1. NREL Report No. } \\
\text { NREL-TP-461-5478 }\end{array}$ & $\begin{array}{l}\text { 2. NTIS Accession No. } \\
\text { DE93018230 }\end{array}$ & 3. Recipient's Accession No. \\
\hline \multirow{2}{*}{\multicolumn{3}{|c|}{$\begin{array}{l}\text { 4. Title and Subtitle } \\
\text { Energy-Efficient Mortgages and Home Energy Rating Systems: A Report } \\
\text { on the Nation`s Progress }\end{array}$}} & $\begin{array}{l}\text { 5. Publication Date } \\
\text { September } 1993\end{array}$ \\
\hline & & & 6. \\
\hline \multicolumn{3}{|c|}{$\begin{array}{l}\text { 7. Author(s) } \\
\text { Barbara C. Farhar and Jan Eckert }\end{array}$} & 8. Performing Organization Rept. No. \\
\hline \multirow{2}{*}{\multicolumn{3}{|c|}{$\begin{array}{l}\text { 9. Performing Organization Name and Address } \\
\text { National Renewable Energy Laboratory } \\
1617 \text { Cole Boulevard } \\
\text { Golden. Colorado } 80401-3393\end{array}$}} & $\begin{array}{l}\text { 10. Project/TaskWork Unit No. } \\
\text { AS } 135440\end{array}$ \\
\hline & & & $\begin{array}{l}\text { 11. Contract (C) or Grant (G) No. } \\
\text { (C) } \\
\text { (G) }\end{array}$ \\
\hline \multirow{2}{*}{\multicolumn{3}{|c|}{ 12. Sponsoring Organization Name and Address }} & $\begin{array}{l}\text { 13. Type of Report \& Period Covered } \\
\text { technical report }\end{array}$ \\
\hline & & & 14. \\
\hline \multicolumn{4}{|l|}{ 15. Supplementary Notes } \\
\hline \multicolumn{4}{|c|}{$\begin{array}{l}\text { 16. Abstract (Limit: } 200 \text { words) } \\
\text { This report summarizes progress throughout the nation in establishing voluntary programs linking home energy } \\
\text { rating systems (HERS) and energy-efficient mortgages (EEMs). These programs use methods for rating the energy } \\
\text { efficiency of new and existing homes and predicting energy cost savings so lenders can factor in energy cost } \\
\text { savings when underwriting mortgages. The programs also encourage lenders to finance cost-effective energy- } \\
\text { efficiency improvements to existing homes with low-interest mortgages or other instruments. The money saved } \\
\text { on utility bills over the long term can more than offset the cost of such energy-efficiency improvements. The } \\
\text { National Collaborative on HERS and EEMs recommended that this report be prepared. }\end{array}$} \\
\hline \multirow{2}{*}{\multicolumn{4}{|c|}{$\begin{array}{l}\text { 17. Document Analysis } \\
\text { a. Descriptors } \\
\text { energy-efficient } \mathrm{m} \\
\text { efficiency } \\
\text { b. Identifiers/Open-E } \\
\text { c. UC Categories } \\
233\end{array}$}} \\
\hline & & & \\
\hline \multirow{2}{*}{\multicolumn{3}{|c|}{$\begin{array}{l}\text { 18. Availability Statement } \\
\text { National Technical Information Service } \\
\text { U.S. Department of Commerce } \\
5285 \text { Port Royal Road } \\
\text { Springfield. VA } 22161\end{array}$}} & $\begin{array}{l}\text { 19. No. of Pages } \\
290\end{array}$ \\
\hline & & & $\begin{array}{l}\text { 20. Price } \\
\text { A13 }\end{array}$ \\
\hline
\end{tabular}

Form No. 0069E (6-30-87) 